\title{
A Force Spectroscopy Setup to Mimic Cellular Interaction Processes
}

\author{
Dissertation \\ zur Erlangung des mathematisch-naturwissenschaftlichen Doktorgrades \\ "Doctor rerum naturalium" \\ der Georg-August-Universität Göttingen
}

vorgelegt von

Bärbel Lorenz

aus Bad Kreuznach

Göttingen, 2012 



\section{Members of the committee}

Prof. Dr. Andreas Janshoff (Reviewer)

Institute of Physical Chemistry, University of Göttingen

Prof. Dr. Ulf Diederichsen (Reviewer)

Institute of Organic and Biomolecular Chemistry, University of Göttingen

Prof. Dr. Jörg Großhans

Institute of Developmental Biochemistry, University of Göttingen

Date of the oral examination: June 26, 2012 

Institute of Physical Chemistry of the University of Göttingen

Supervisor: Prof. Dr. Andreas Janshoff 

Hereby, I certify that my doctoral thesis entitled "A Force Spectroscopy Setup to Mimic Cellular Interaction Processes" has been written independently and with no other sources and aids than quoted.

\section{Bärbel Laxeuz}

Bärbel Lorenz

Göttingen, May 23, 2012

Copenhagen/Dublin, June 19, 2013 (revised version) 



\section{Table of Contents}

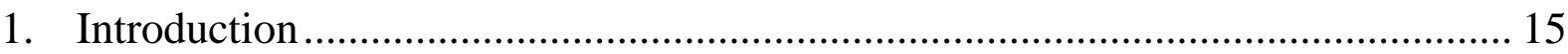

1.1 Cells and their environment - cell-cell and cell-substrate interactions...........................15

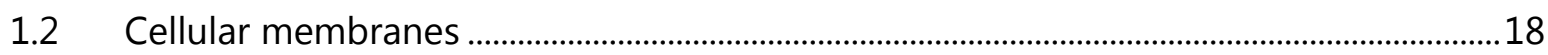

1.3 Membrane model systems ………………......................................................................19

$1.4 \quad$ Tailoring artificial membrane surfaces ................................................................................21

1.5 Surface functionalization for biological applications .......................................................23

1.6 Force spectroscopy measurements of biological interactions.............................................25

1.6.1 Force spectroscopy of cell-to-substrate-adhesion ………………………………………………....2 27

1.6.2 Force spectroscopy of cell-cell interactions.....................................................................................2 29

1.6.3 Force spectroscopy of membrane tethers............................................................................................. 29

1.6.4 Force spectroscopy of fusion processes............................................................................................ 31

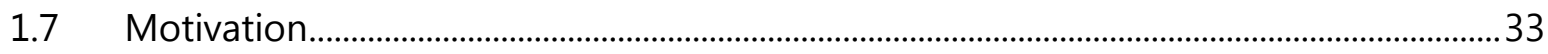

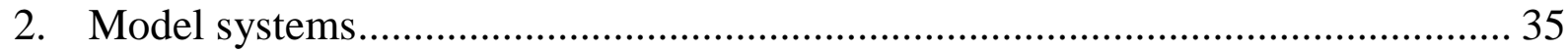

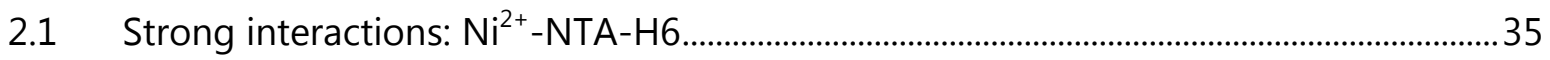

2.2 Weak interactions: Disaccharides derived from Microciona prolifera..................................37

$2.3 \quad$ Coiled-coil forming peptides $i$-E3Cys and $i$-K3Cys...............................................................40

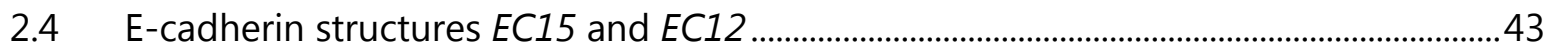

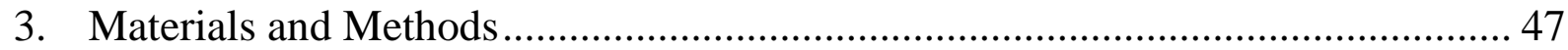

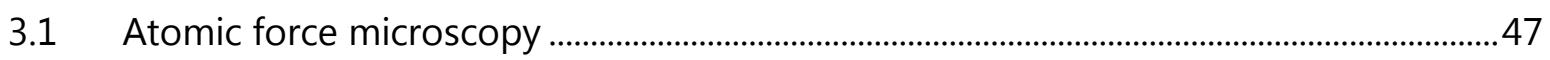

3.1.1 Imaging modes .................................................................................................................................... 51

3.1.2 Force spectroscopy ……………………………………………………………………………. 52

3.2 Fluorescence microscopy …………………..........................................................................54

3.3 Reflectometric interference spectroscopy …….....................................................................58

3.4 Membrane probe microscopy: preparation of probe system ………………......................59

3.4.1 Manufacturing of colloidal probe cantilevers …………………………………………………....... 59 
3.4.2 Preparation of small unilamellar vesicles 60

3.4.3 Preparation of membrane-coated probes and substrates 61

3.4.4 Membrane functionalization 62

3.4.5 Membrane probe spectroscopy measurements 63

3.5 Membrane probe spectroscopy of the $\mathrm{Ni}^{2+}-\mathrm{NTA}-\mathrm{H} 6$ interaction....................................66

3.5.1 Synthesis and purification of $\mathrm{H} 6$ peptides 66

3.5.2 Functionalization of membranes with $\mathrm{Ni}^{2+}-\mathrm{NTA}$ and $\mathrm{H} 6$ residues 67

3.5.3 Membrane probe spectroscopy measurements with $\mathrm{Ni}^{2+}$-NTA-H6-doped membranes..... 68

3.6 Membrane probe spectroscopy of (non-) sulfated disaccharides 69

3.6.1 Synthesis and purification of Microciona prolifera disaccharides. 69

3.6.2 Functionalization of membranes with thiol-linked disaccharides. 69

3.6.3 Membrane probe spectroscopy measurements with (non-) sulfated disaccharidesfunctionalized membranes. 71

3.7 Membrane probe spectroscopy of the $i$-E3Cys/i-K3Cys interaction 72

3.7.1 Synthesis and purification of the peptides $i$-E3Cys and $i$-K3Cys ............................................... 72

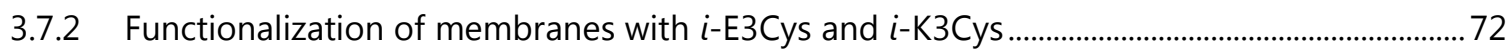

3.7.3 Membrane probe spectroscopy with $i$-E3Cys/i-K3Cys-doped membranes............................... 73

3.8 Single molecule force spectroscopy of cadherin interactions ............................................. 74

3.8.1 Expression and purification of SNAP-tagged proteins EC15 and EC12 ……….......................... 74

3.8.2 Functionalization of gold substrates with $E C 15$ and $E C 12$ proteins............................................75

3.8.3 Single molecule force spectroscopy of the homomeric cadherin interaction .78

4. Probing receptor-ligand interactions with an atomic force microscope

5. Membrane probe spectroscopy 87

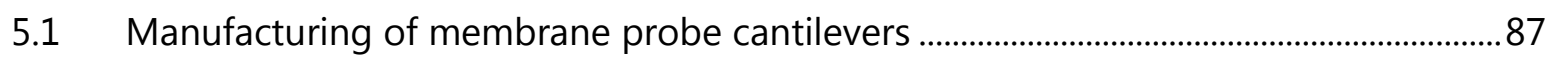

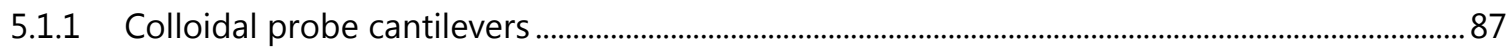

5.1.2 Manufacturing of membrane probe cantilevers ............................................................................ 88

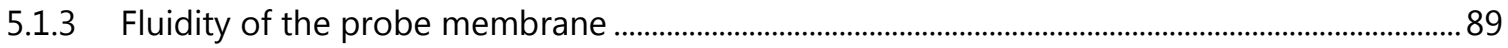

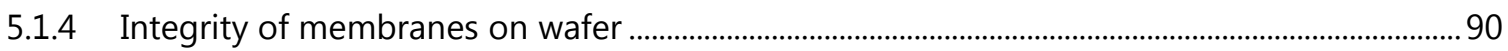

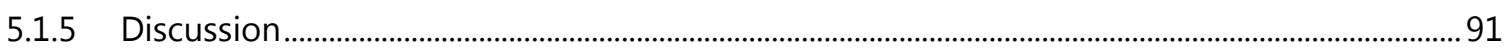


5.2 Technical aspects of membrane probe spectroscopy measurements 93

5.3 The strength of the $\mathrm{Ni}^{2+}-\mathrm{NTA}-\mathrm{H} 6$ interaction system .94

5.3.1 The membrane probe setup for probing the $\mathrm{Ni}^{2+}-\mathrm{NTA}-\mathrm{H} 6$ interaction. 94

5.3.2 Functionalization of membranes with $\mathrm{Ni}^{2+}$-NTA and $\mathrm{H} 6$. 94

5.3.3 Adhesion force and work of adhesion of the $\mathrm{Ni}^{2+}-\mathrm{NTA}-\mathrm{H} 6$ interaction. 94

5.3.4 Determination of formed ligand-receptor bonds. 97

5.3.5 Wearing-off of the system .98

5.3.6 The approach curve - hemifusion instead of docking? 99

5.3.7 Stochastic bond breakage or membrane fusion. 100

5.3.8 Lipid tethers are formed between the membranes. . .101

5.3.9 Model perception 106

5.4 Self-recognition between disaccharides derived from Microciona prolifera 108

5.4.1 Probing the homomeric Microciona prolifera interaction with a membrane probe setup 108

5.4.2 Functionalization of membranes with disaccharides derived from Microciona prolifera ..109

5.4.3 Necessity of the sulfated epitope and of calcium ions for the self-recognition process ...110

5.4.4 Kinetics of the disaccharide interaction and cluster formation .111

5.4.5 Membrane probe spectroscopy of weak disaccharide interactions .115

5.5 Coiled-coil formation of $i$-E3Cys/i-K3Cys lipopeptides 116

5.5.1 Probing the $i$-E3Cys/i-K3Cys interaction with a membrane probe setup. 116

5.5.2 Functionalization of membranes with $i$-E3Cys and $i-K 3$ Cys 116

5.5.3 Impact of lipopeptide concentration, dwell time, and loading rate on interaction forces between $i$-E3Cys/i-K3Cys-functionalized membranes.

5.5.4 Estimation of the number of $i$-E3Cys/i-K3Cys coiled-coils involved in the contact zone ..121

5.5.5 Impact of peptide clustering on membrane docking efficiency. .122

6. Towards cellular interactions: Single molecule force spectroscopy of cadherin-mediated interactions

6.1 Characterization of cadherin-functionalized surfaces.

6.2 Single molecule force spectroscopy of the homomeric cadherin interaction 126

6.2.1 Interaction forces between EC15/EC15 and EC12/EC12. .126

6.2.2 Contact time dependence of the EC15/EC15 interaction 129 
6.2.3 Loading rate dependence of the EC15/EC15 interaction 129

6.2.4 Impact of trans/cis binding for the EC15/EC15-interaction 131

6.3 Quantification of cadherin monomer-cell interactions 133

7. Conclusions

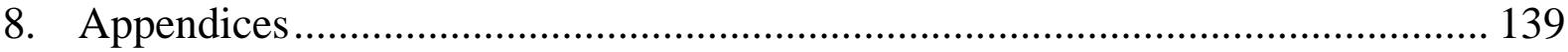

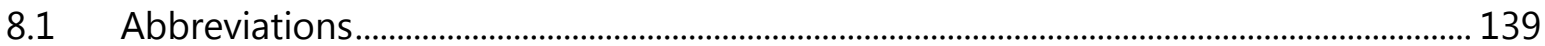

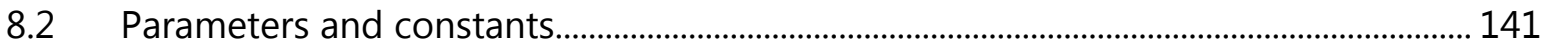

8.3 Supplementary information about probed model systems............................................. 142

8.3.1 Characterization of the $\mathrm{Ni}^{2+}-\mathrm{NTA}-\mathrm{H} 6$ interaction .........................................................................142

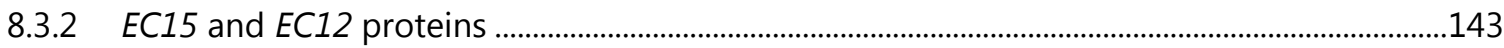

8.3.3 (Non-)sulfated Microciona prolifera disaccharides..........................................................................144

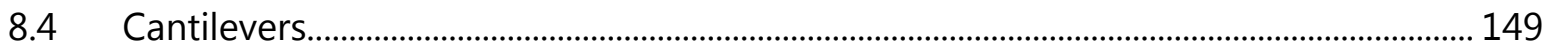

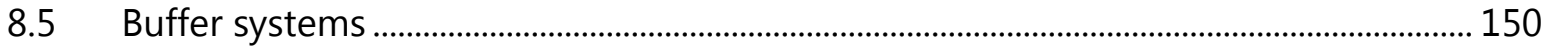

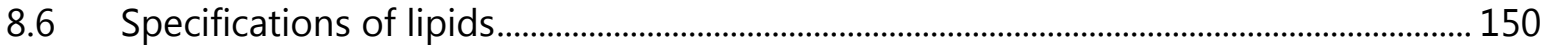

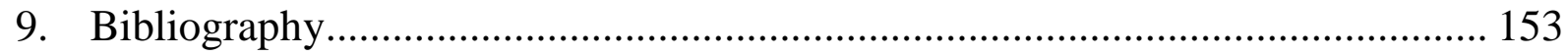

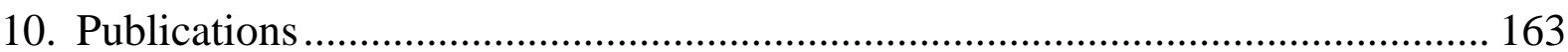




\section{Abstract}

The main objective of this thesis was to establish a biophysical model system that permits to quantify forces involved in cell-cell contacts and cell-substrate-adhesion in a native-like fashion. For this purpose colloidal probe microscopy was modified to mimic two opposing membranes, a situation very much like that encountered in cell-cell recognition. Membrane coated probes allow to capture the impact of lateral mobility, multiple bonds, and merging of bilayers as encountered in fusion events (Scheme 1.1).

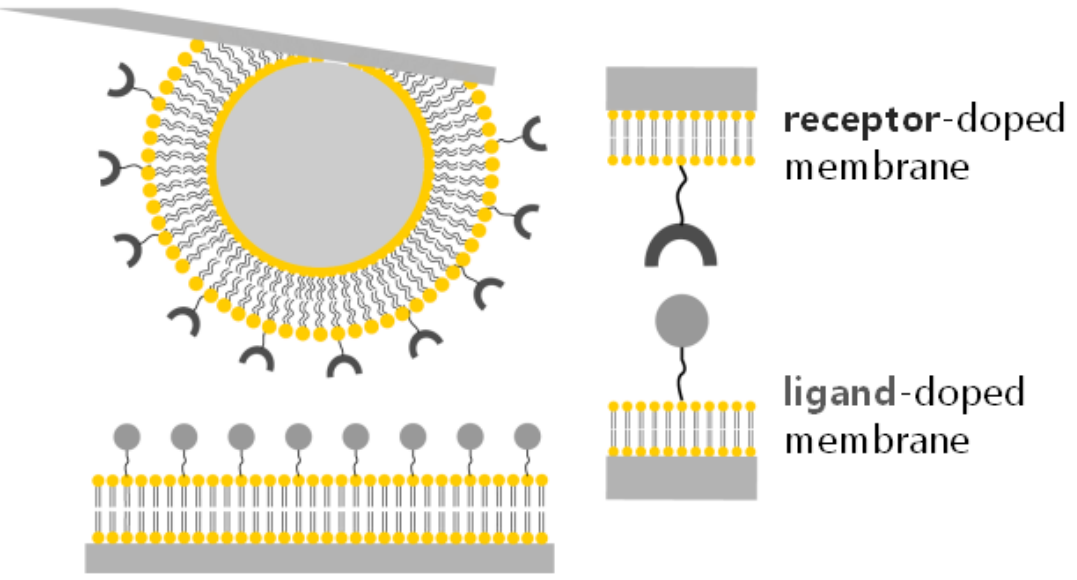

Scheme 1.1: Principle of a membrane probe spectroscopy setup. A ligand-doped membrane is brought into contact with a receptor-functionalized membrane in order to probe mechanics, kinetics, and thermodynamics of membrane interactions in a cell-mimicking manner.

In the first part, the strong non-covalent interaction between His-tagged peptides embedded in a lipid bilayer and Ni-NTA bearing lipids in the opposing membrane were probed. Hemifusion as well as full fusion events were frequently observed depending on the compression forces. Upon retraction from the surface, membrane nanotubes were formed that allowed to carry out force clamp measurements. The constant force of a tether permits to measure the lifetime of individual bonds within the contact zone.

The second part was devoted to weak non-covalent interactions playing a pivotal role in the formation of focal contacts or cell-cell recognition. Many of these bonds in parallel provide the necessary strength to attach cells to surfaces or other cells forming tissue. Early metazoae such as sponges use carbohydrate moieties to form reversible bonds between each other a prerequisite for self-recognition, a crucial advantage in evolution. 
Membrane-based colloidal probe microscopy was used to quantify the forces between sulfated carbohydrates as they are found in the marine sponge Microciona prolifera organized in small clusters. The interaction was found to be highly calcium dependent and reversible.

In the third part of the project, the heterodimeric coiled-coil interaction between peptides, which serve as minimum model for the SNARE-mediated membrane fusion, was studied. We found that the membrane interaction forces are significantly smaller than expected. By performing topographical imaging and photobleaching experiments of the membrane, we could attribute the low adhesion forces to a lipopeptide cluster formation prior to the formation of the coiled-coils. We conclude that efficient membrane docking, which is the prerequisite for fusion events, does not only depend on the concentration of peptides on the surface but is decisively controlled by the lateral organization of peptides in the membranes.

The fourth part of the thesis dealt with interactions found between highly evolved eukaryotic cells. The homomeric recognition of E-cadherins from L-cells was probed on the level of single molecule to shine light on the assembly scheme used by cells to ensure a firm connection between them. Force spectroscopy revealed two distinguishable modes of interactions that could be presumably attributed to cis-interaction between adjacent cadherins. While the homomeric EC12 recognition was barely detectable, EC15 displayed forces beyond the background suggesting that the length of the molecule plays an important role.

In conclusion, a versatile lab-on-a-probe setup has been introduced that allows studying a large variety of interactions between membranes and that is essentially mimicking cell-cell interactions with reduced complexity. 


\section{Introduction}

\subsection{Cells and their environment - cell-cell and cell-substrate interactions}

Understanding cellular interaction processes is pivotal for tissue engineering, implant design, and for the control of pathogenesis of diseases like viral infections or cancer. Hence, research on cell-cell, cell-virus and cell-substrate interaction sites is of invaluable importance for modern life sciences (Figure 1.1). ${ }^{[1,2]}$ Loss of cellular interactions such as interactions between cadherins results, for instance, in an increased metastatic potential of cancer cells due to the lack of intercellular docking sites.

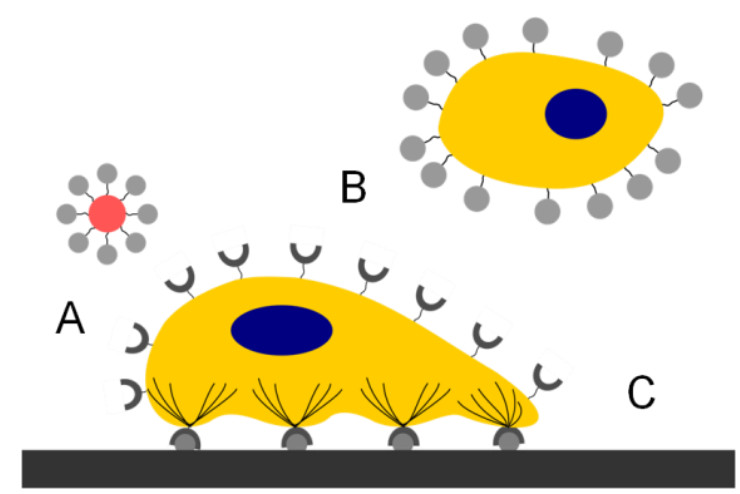

Figure 1.1: Cellular interaction processes addressed in this study. ${ }^{1}$ The aim of the project was the establishment of a biophysical model system, which serves to quantify aspects of cell-virus (A), cell-cell (B), and cell-substrate $(C)$ interactions.

Moreover, controlled attachment and detachment regulate leukocyte binding to endothelium during inflammation processes. The key to cope with viral infections is to understand the reactions that take place at the first contact of cell and virus. Furthermore, the establishment of cell to substrate contacts is of crucial importance for the attachment of cells to connective tissue or the inclusion of rigid material like bone or cartilage into surrounding tissue. The Marfan syndrome for instance is characterized by a dysfunctionality of connective tissue resulting in aorta and skeleton malfunctions ${ }^{[3]}$ In these entire pathological processes vital cell signaling processes are influenced due to the lack or the occurrence of specific interactions between the cell and its periphery. Most relevant structures on cell surfaces are lectins, cadherins, and integrins as well as polysaccharide-bearing lipids.

\footnotetext{
${ }^{1}$ Schematic is not drawn to scale. All schematics are drawn by B. L. unless otherwise stated. 


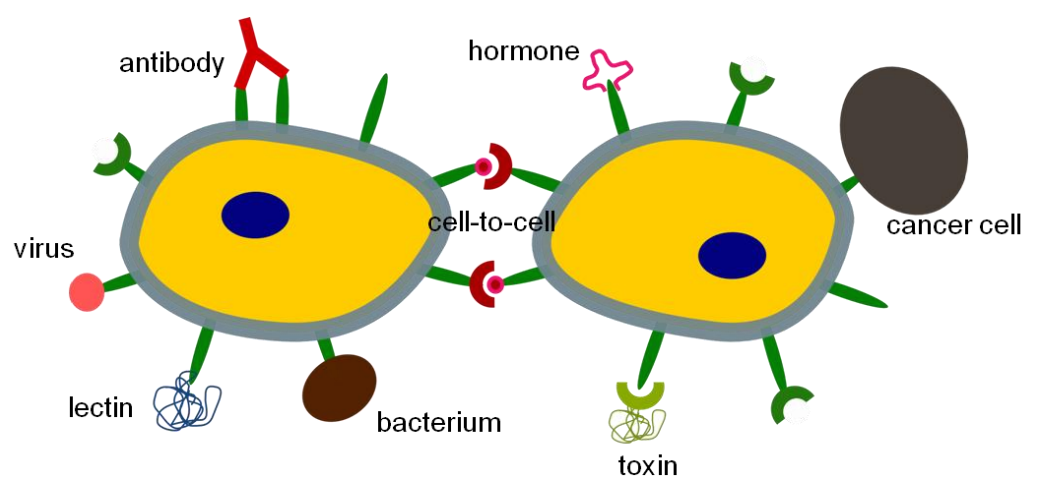

Figure 1.2: An intercellular interaction process of two cells each of which can be subjected to antibody, virus, bacterium, lectin, hormone, cancer cell, and toxin binding via cell-surface receptors (green symbols). Intracellular signal cascades are activated upon binding of ligands.

Cell surface structures act as receptors for antibody and hormone binding, an important process for the protection and the development of a cell (Figure 1.2). However, cells are exposed to various challenges during their lifetime: they are subjected to docking processes of viruses, toxins, bacteria and cancer cells, which can have pathological consequences for the tissue. ${ }^{[4]}$ Non-covalent adhesion forces acting between those receptors and their ligands are valuable in order to allow for cell locomotion but can be compensated by molecule clustering, which results in a strengthening of cellular interaction.

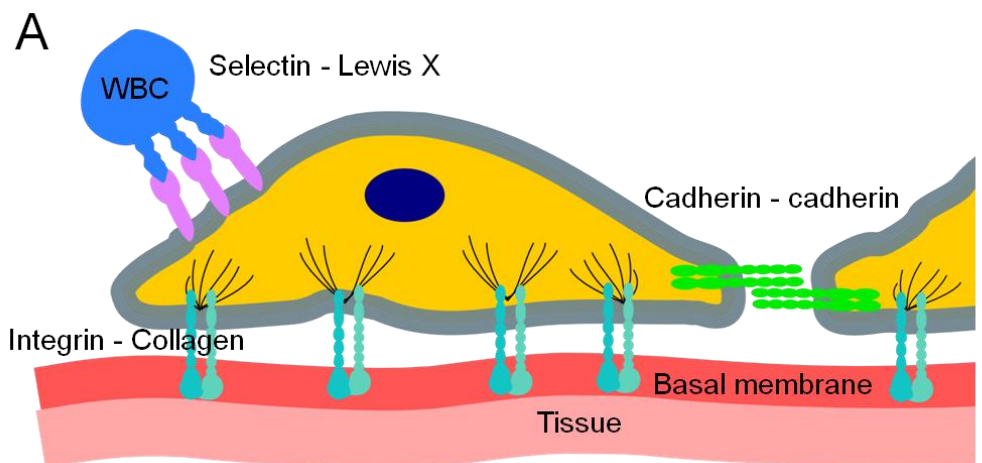

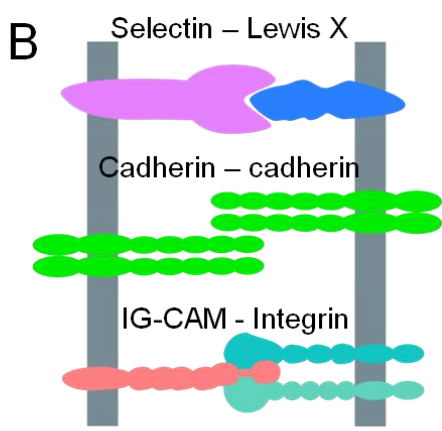

extracellular space

Figure 1.3: Major families of cell surface receptors involved in cellular adhesion processes. ${ }^{2}$ Cell-cell adhesion is mediated by cadherins and selectins, whereas cell-substrate adhesion is mediated by integrin structures. Cadherins (green structures) mediate contacts between endothelial cells via a homomeric coupling of their ectodomains. Selectins (blue) control the coupling of white blood cells onto the walls of blood vessels by recognizing polysaccharides on glycoproteins such as the Lewis $X$ factors (violet). Integrin structures (petrol) control cell-matrix interactions via coupling to collagen as well as cell-cell interactions via coupling to immunoglobulin structures. In contrast to selectins and integrins, which act in a heteromeric fashion, cadherins perform a homomeric interaction.

\footnotetext{
${ }^{2}$ Images adapted from 5. Sackmann, E. and Goennenwein, S. (2006). "Cell Adhesion as Dynamic Interplay of Lockand-Key, Generic and Elastic Forces." Progress of Theoretical Physics Supplement 165: 78-99.
} 
In order to functionally couple a cell to a substrate or two a second cell, specific interaction sites need to be present in the cell surface. Figure 1.3 demonstrates cellular surface receptors, which allow for communication of the cell with its environment. ${ }^{[5]}$ In the case of epithelial cell monolayers, tight junctions (Figure 1.4-B), adherence junctions (Figure 1.4-C), desmosomes (Figure 1.4-D), and gap junctions (Figure 1.4-E) coordinate communication and adhesion between cell walls.

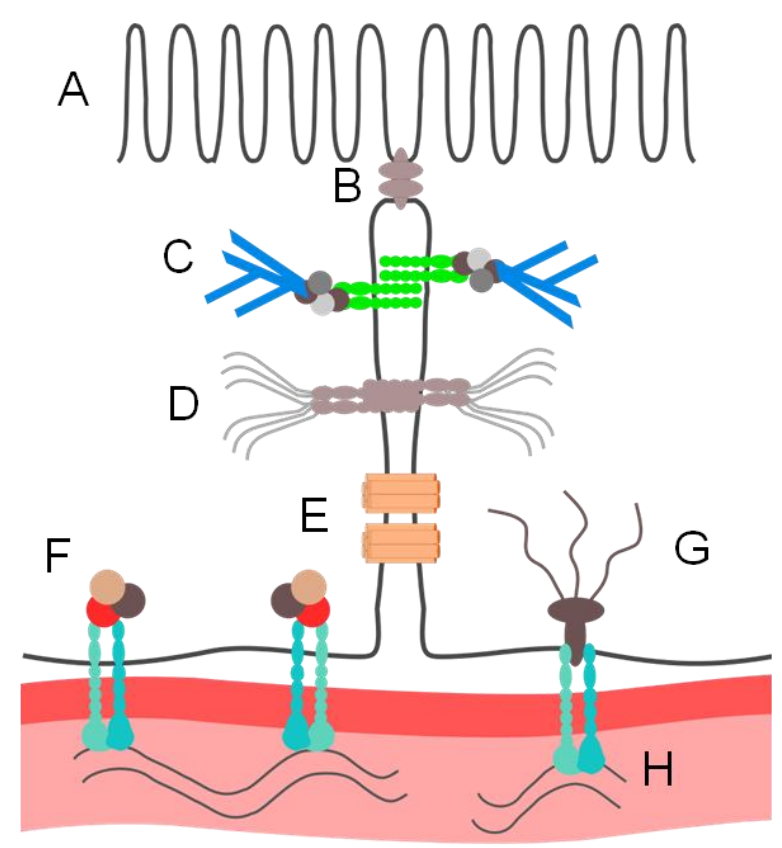

Figure 1.4: Intracellular interactions in an epithelial cell monolayer. ${ }^{3}$ The apical cell membrane is characterized by microvilli structures (A) enlarging the surface area available for the cells' uptake of nutrients. The lateral and basal membranes form borders to adjacent cells and the substrate, respectively. Tight junctions (B), adhesion junctions (C), desmosomes (D) and gap junctions (E) are functional units on the lateral membranes, whereas focal contacts (F) and hemidesmosomes (G) in the basal membrane are relevant for cellsubstrate interaction processes $(\mathrm{H})$. Cytoskeletal components such as intermediated filaments (hemidesmosomes, desmosomes) and actin (adhesion junctions, focal contacts) are connected to these units. Gap junctions control the transport of small molecules, whereas tight junctions have a fence function.

Tight junctions seal the intercellular space from the lumen of the tissue as they build barriers between the apical and the basolateral membranes of the cell. Gap junctions control the transport of small molecules between the cytosol of two adjacent cells, whereas the other structures shown on the lateral and the basal side of the cell act as mechanical transducers between the cells. Adherence junctions and focal contacts (basal cell membrane Figure 1.4-F) are coupled to actin filaments, whereas hemidesmosomes (basal membrane, Figure 1.4-G)

\footnotetext{
${ }^{3}$ Image adapted from 6. 6. Wegener, J. (2001). Cell Junctions. eLS, John Wiley \& Sons, Ltd.
} 
and desmosomes are connected to intermediate filaments. ${ }^{[6]}$ Key structures of these functional clusters are cadherin (adherence junctions, desmosomes) and integrin (focal contacts, hemidesmosomes) structures. Immunoglobulins, selectins, cadherin and integrin molecules belong to the four major classes of cell adhesion molecules (CAM) depicted in Figure 1.3-B. ${ }^{4[5]}$ Cadherins are $\mathrm{Ca}^{2+}$-dependent proteins ("calcium-dependent adhesion") involved in adherence junctions and desmosomes structures in a homomeric kind (Figure 1.3). Cell-substrate interactions are mediated by a heteromeric interaction between integrin and RGD (arginineglycine-aspartic acid) sequences on fibronectin or immunoglobulin structures (Figure 1.4-H).

\subsection{Cellular membranes}

All communication processes of cells with their surroundings are processed by cellular membranes consisting of self-assembled fluid lipid matrices with embedded proteins (Fig-1.6). Besides protein conformation changes lipid rafts are also reported to control functionality between the intracellular and the extracellular compartment (Fig. 1-6 B/C). Intracellular communication is managed via endocytosis and exocytosis requiring controlled membrane morphology changes such as diffusion of compounds as well as fusion of membranes. Transportation of cargo can be realized by the formation of lipid tethers between two cells. ${ }^{[7]}$
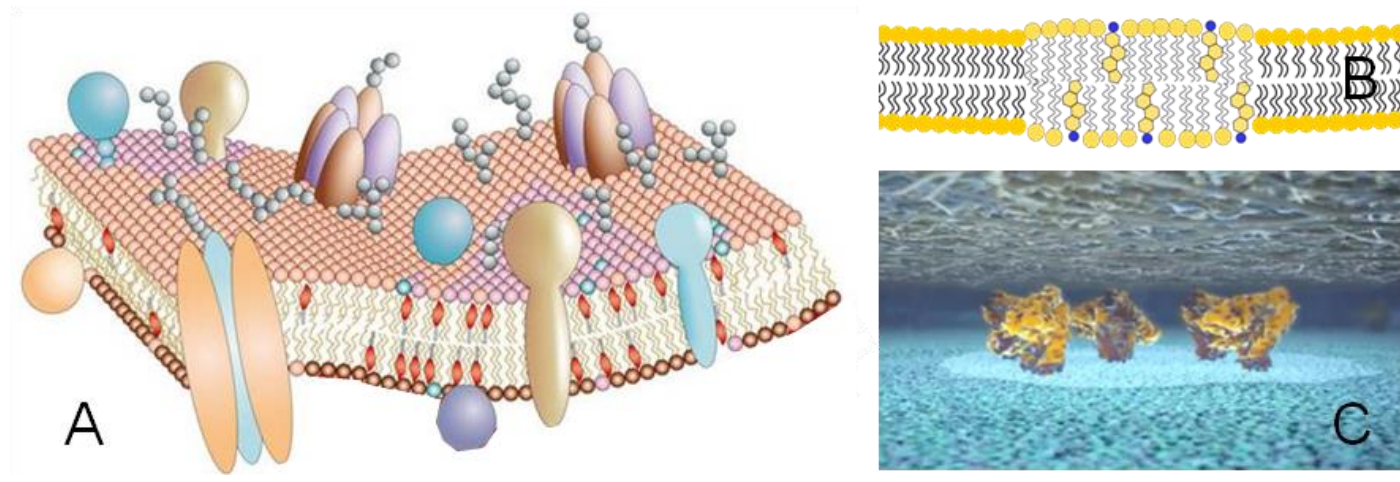

Figure 1.5: Cell membrane setup. A: The fluid mosaic model consisting of a membrane including glycosylated integral proteins and glycolipids. ${ }^{5}$ B, C: Schematics of a membrane raft embedded in a matrix membrane. B: The raft consists of sphingomyelin and cholesterol molecules (yellow structures with blue heads). C: The functionality of the raft region is given by proteins embedded in the membrane. ${ }^{6}$

\footnotetext{
${ }^{4} \mathrm{~A}$ list of abbreviations can be found in chapter 8.1 on page 126.

${ }^{5}$ Image of Pietzsch, J., "The fluid-mosaic model of the cell membrane", nature.com, Nov 26, 2012, reprinted as a slightly modified version with permission of Nature Publishing Group.

${ }^{6}$ Image is a snapshot taken from the video "The Inner Life of the Cell", Harvard Biovision, Leigh Stimolo, Alain Viel.
} 
In the last century, several membrane models have been developed. A simple two lipid monolayer model postulated by Gorter and Grendel in 1925 has been replaced by the fluid mosaic model established by Singer and Nicolson in 1972, which proposes the inclusion of membrane proteins as well as glycolipids into the lipid bilayer (Figure 1.5-A). ${ }^{[8,9]}$ The fence model envisioned by Kusumi in 1993 states that the diffusion of proteins in the membrane is limited by cytoskeletal anchors. ${ }^{[10]}$ Sackmann emphasizes the relevance of the glycocalyx on cellular surfaces and of the cytoskeleton inside cells. ${ }^{[1]}$
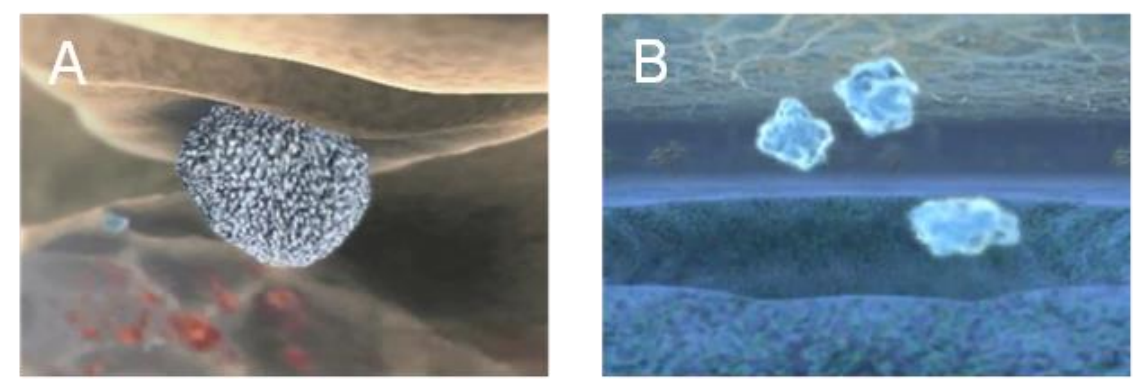

Figure 1.6: Processes involving membrane functionality in vivo. A: leukocyte adhesion to an endothelial cell layer. 6 B: endocytosis of proteins into the cytoplasm involving fusion of membranes. ${ }^{6}$

In 1997, Simons and Ikonen proposed the raft hypothesis arguing for the existence of small functional protein clusters in cellular membranes playing a decisive role for the functionality of the cellular membrane (Figure 1.5-B, C). Figure 1.6 shows two cellular processes involving different membrane functions: a leukocyte manages to bind to an endothelial cell wall $(A)$, and proteins are segregated via exocytosis (B). The overall goal of life sciences is to explain these processes by creating model systems allowing for the study of certain aspects of these processes.

\subsection{Membrane model systems}

A popular approach to analyze certain aspects of cellular interactions is to use artificial membrane model systems as they serve for a large range of applications. Lipid aggregates are easy to form, handle and to modify. Due to their amphiphilic nature (Figure 1.11-A) lipid molecules self-assemble into aggregates above the critical micelle concentration (CMC, $\sim 10^{-10}$ mol for phospholipids) due to the hydrophobic effect. ${ }^{[12]}$ They provide an excellent resource to investigate processes such as membrane-membrane assembly, i.e. aggregation, hemifusion or full fusion in a quantitative manner under controlled conditions and de- 
fined composition. Figure 1.7 shows a compilation of artificial membrane setups. Vesicles in different sizes (Figure 1.7 -A) can be spread to give solid supported lipid bilayers (Figure 1.7B) or lipid bilayers tethered by a polymer cushion (Figure $1.7-C) .{ }^{[13,14]}$ For producing black lipid membranes shown in Figure 1.7-D, one paints lipid molecules across the hydrophobic substrate. ${ }^{[15]}$
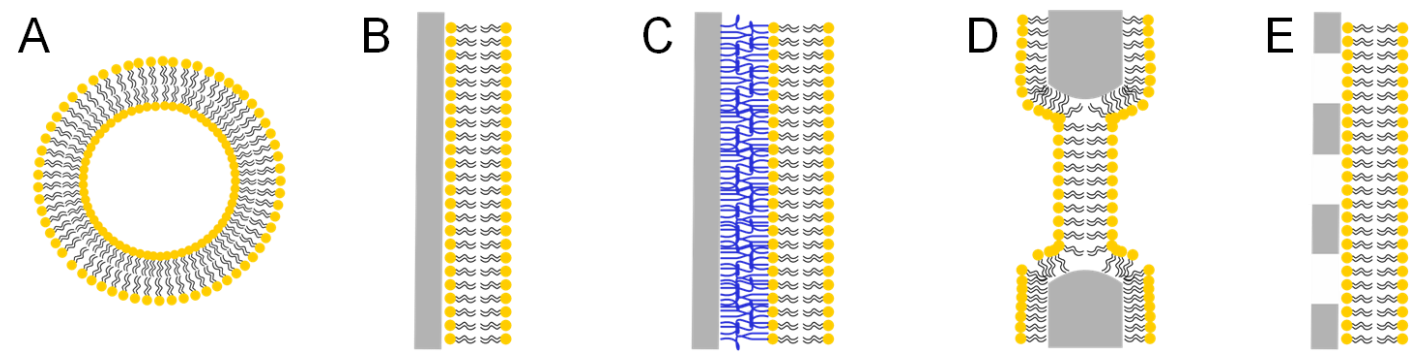

Figure 1.7: Popular lipid bilayer model systems. Vesicles (A) are commonly used to perform vesicle fusion assays, to track domain formation and to form solid-supported lipid bilayers (SSLBs, B and C (tethered by a polymer cushion)). SSLBs are commonly used to detect ligand binding or to study domain formation. Black lipid membrane systems (D) and membranes spread on porous substrates (E) allow for the detection of transportation processes through the membrane. Moreover, membrane indentation experiments can be performed on setups as depicted in $\mathrm{E}$.

Coverage of porous substrates (Figure 1.7-E) can be achieved by spreading giant unilamellar vesicles. ${ }^{[16]}$ Vesicles are analyzed to determine the impact of membrane stiffness, lipid domains, and to quantify fusion processes, whereas solid supported lipid bilayers (SSLB) are mainly used to perform binding studies. ${ }^{[17,18]}$
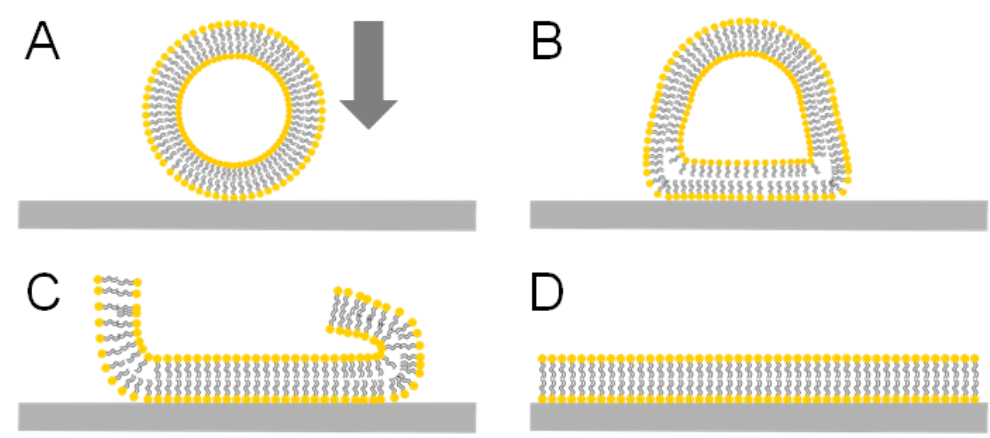

Figure 1.8: Vesicle spreading produces solid-supported lipid membranes. ${ }^{7}$. Vesicles settle down on hydrophilic surfaces (A to B) and burst (C) forming a continuous SSLB. Whether the inner or the outer leaflet of the vesicle gets in contact with the substrate is still a matter of debate.

SSLB preparation can be achieved by using the Langmuir-Blodgett technique or the vesicle spreading technique (Figure 1.8).

\footnotetext{
${ }^{7}$ Adapted from 11. Raedler, J., et al. (1995). "Phenomenology and Kinetics of Lipid Bilayer Spreading on Hydrophilic Surfaces." Langmuir 11(11): 4539-4548, usage free of charge
} 
Vesicle spreading can be stimulated by appropriate functionalization of vesicles and surfaces. ${ }^{[19]}$ Black lipid membranes and pore spanning membranes allow for a combination of both setups as they offer solid supported as well as free standing areas of the membrane. This setup in turn, can also be used to determine membrane stiffness, to quantify transport through the membrane and to quantify fusion processes.

\subsection{Tailoring artificial membrane surfaces}

The composition of native cell membranes is complex and optimized with regard to the function that the cells have in the respective tissue. Geometry and charge of lipid molecules as well as asymmetry of the lipid bilayer play a crucial role for the characteristics of a membrane. ${ }^{[20-23]}$ The outer leaflet of a cellular membrane is normally rich in phosphatidylcholine (PC)-bearing lipids and sphingomyelin and has, in turn, a neutral net charge. The intracellular membrane monolayer, in contrast, is composed of negatively charged phosphatidyl-serine (PS)-bearing lipids and phosphatidylethanolamine ( $P E$, neutral) rendering the leaflet negatively charged. ${ }^{[23]}$

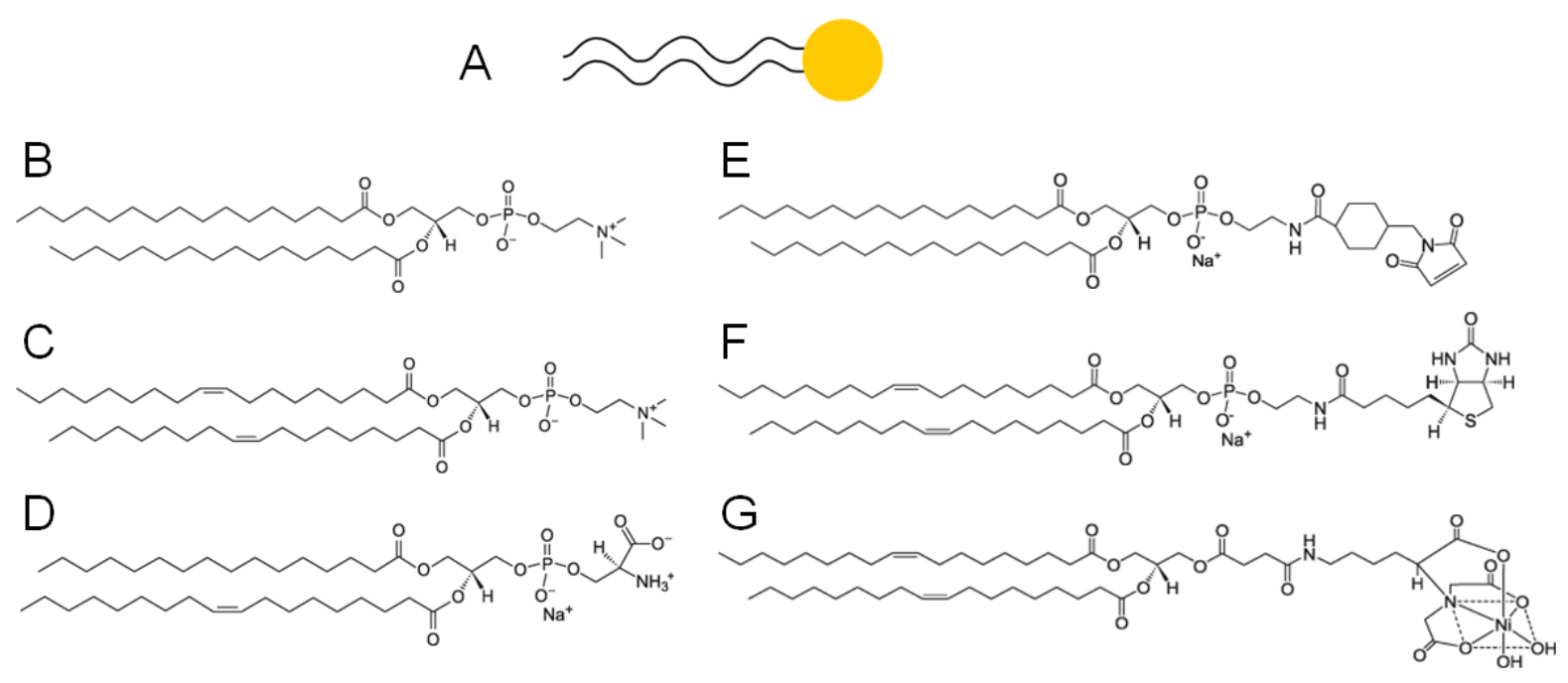

Figure 1.9: Headgroup modification in lipids allows for covalent immobilization of receptors in an artificial membrane. A: Amphiphilic nature of a phospholipid. B-G. ${ }^{8}$ Matrix lipids such as 1,2-dipalmitoyl-snglycero-3-phosphocholine (DPPC, B), 1,2-dioleyl-sn-glycero-3-phosphocholine (DOPC, C), or 1-palmitoyl2-oleoyl-sn-glycero-3-phospho-L-serine (POPS, D) can be equipped with functionalized lipids such as maleimide-terminated ones (E), biotin-labeled ones (F), or $\mathrm{Ni}^{2+}$-NTA-tagged ones (G) in a certain percentage. The lipids can then be used to bind molecules tagged with thiol, avidin, or histidine residues.

\footnotetext{
${ }^{8}$ Representational structures shown above kindly supplied by Avanti Polar Lipids, Inc., www.avantilipids.com.
} 
There are different approaches to investigate cellular membranes. For example, isolated basal cell membranes can be prepared from confluent epithelial cell layers by using a lysing-squirting protocol. ${ }^{[24,25]}$ Simonsson demonstrated that continuous lipid bilayers derived from cell membranes can be spread at the border of artificial membranes for spatial molecular manipulation. ${ }^{[26]}$

When using completely artificial lipid membranes as a bottom-up approach, a toolbox of commercially available lipids (Figure 1.9) can be used to design artificial membranes in order to get closer to the setup of a native membrane (Figure 1.11). Receptor lipids (Figure 1.9-E, F, G) can be embedded into matrix lipids such as 1,2-dipalmitoyl-sn-glycero-3-phosphocholine (DPPC), 1,2-dioleyl-sn-glycero-3-phospho-choline (DOPC), or 1-palmitoyl-2-oleoyl-snglycero-3-phos-pho-L-serine (POPS) (Figure 1.9-B, C, and D, respectively). The depicted receptor lipids can be used to immobilize thiol-linked molecules (E), to bind avidin-linked molecules (F) or His-tagged molecules (G).
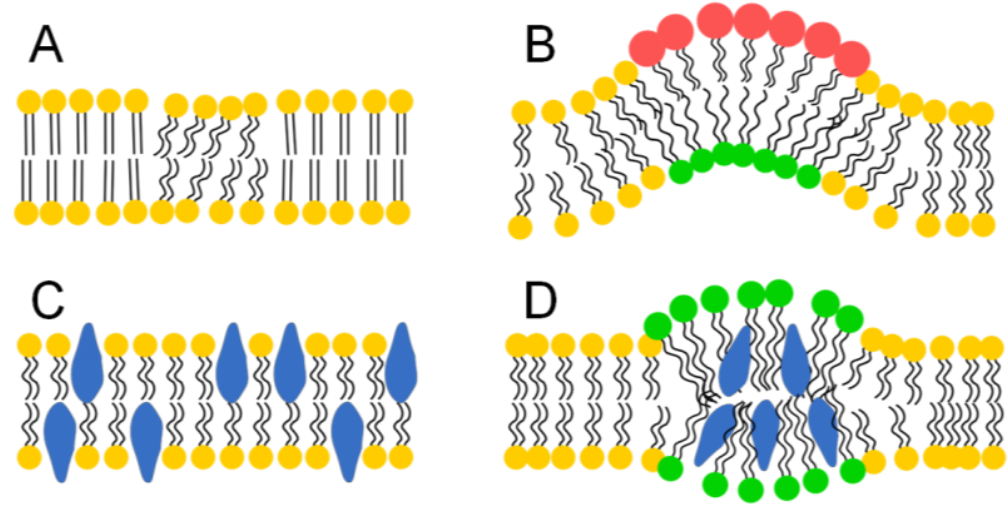

Figure 1.10: Changes in membrane properties due to lipid composition. Mixtures of gel phase and fluid phase lipids phase separate as shown in A. Spontaneous membrane curvature (B) can be induced by incorporating certain molecules into a lipid matrix. Stiffness and fluidity of membranes (C) as well as the tendency to form lipid rafts (D) can be altered as well.

By using different kinds of gel phase or fluid phase lipids as a matrix in combination to headgroup-modified lipids embedded in this matrix one can tune the fluidity, phase behavior (Figure 1.10-A), spontaneous curvature and bending properties (Figure 1.10-B), as well as membrane thickness, compressibility, its charge and adhesive properties. Integration of cholesterol molecules is reported to stiffen a membrane (Figure 1.10-C) and, in combination with sphingolipids, reported to be enriched in small functional domains, the lipid rafts (Figure 1.10-D). 
To further build up the membrane assembly, it is conceivable to equip lipids with different kinds of ligands and receptors. Figure 1.11 shows how to tailor functional SSLBs by equipping them with ligand and receptor molecules in a step-wise manner.

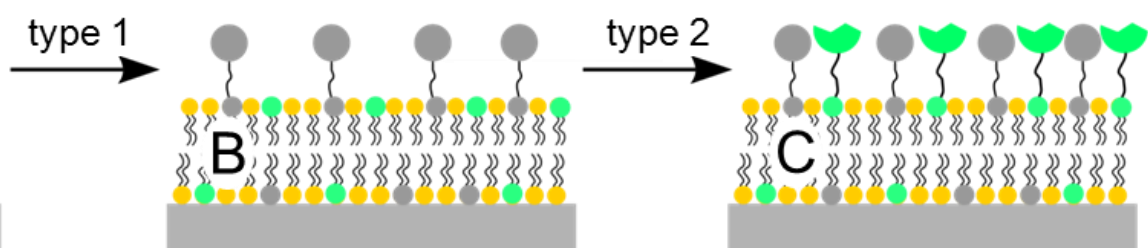

Figure 1.11: Tailoring of functionalized membranes. A: membrane doped with two classes of receptor lipids. B: membrane functionalized with type 1 ligand molecules binding to receptor lipid class 1 . C: membrane functionalized with type 1 and type 2 ligands, which bound to receptor lipid class 2 .

By equipping a simple lipid bilayer with a variety of functional lipids and proteins a cellular membrane mimic can be established step-by-step.

\subsection{Surface functionalization for biological applications}

The study of particular processes requires the covalent immobilization of receptors and ligands on hard surfaces. General surface functionalization strategies are silanization for silicon surfaces and soft lithography for metal surfaces developed by Whitesides et al. ${ }^{[27]}$
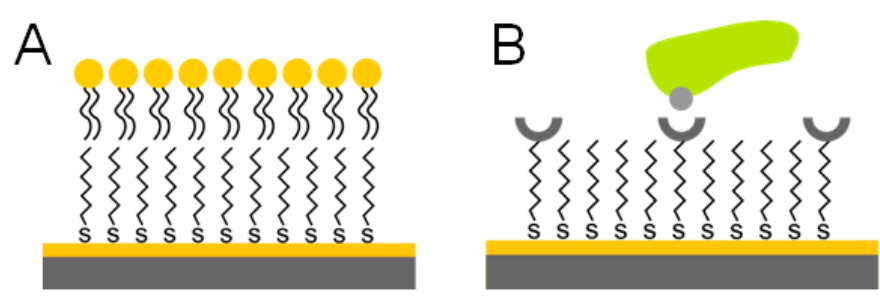

Figure 1.12: Two thiol chemistry applications. A self-assembled thiol monolayer (SAM) on a gold surface can be used for the formation of alkane-lipid hybrid membranes (A) and for the covalent immobilization of specifically tagged proteins (B). Thiol molecules are available with a large variety of receptor entities. For protein binding, they are usually combined to matrix thiols, which prohibit unspecific binding. In the case of membrane hybrids on thiol monolayers it is recommendable to use pure carbon chain thiols.

It makes use of thiols that self-assemble into homogeneous monolayers on gold substrates by chemisorption. ${ }^{[28,29]}$ Depending on the nature of thiols bound, the surfaces can be used to immobilize any kind of molecule via receptor-ligand coupling (Figure 1.12-A) and as substrates for membrane hybrids (Figure 1.12-B). 
If patterned surfaces are required, several methods are available one of which is the microcontact printing $(\mu \mathrm{CP})$ method (Figure 1.13). ${ }^{[30]}$

A

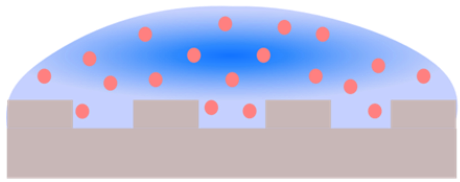

C
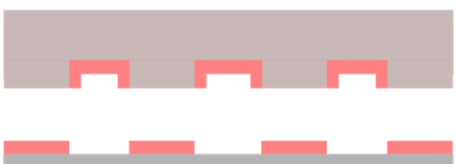

B

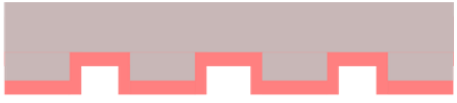

$\mathrm{D}$

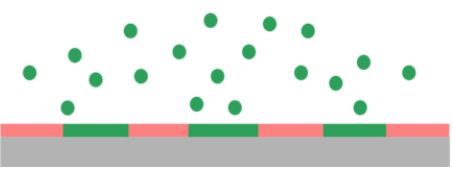

Figure 1.13: Microcontact printing $(\mu \mathrm{CP}) .^{9}$ A patterned elastomeric stamp fabricated by curing a liquid prepolymer on a silicon master (Figure 1.14) and is incubated with molecular ink (A). It is then used to stamp the ink on a flat surface (B to $C$ ) to give a patterned surface functionalized with a specific class of molecules. The space between the stamped regions can be back-filled with a second class of molecules (D).

With this technique molecules in aqueous or organic solvent can be printed onto metal, metal oxide, glass, or plastic surfaces by using a PDMS (polydimethylsiloxane), POP (poly(oxyethylene glycol)polymer or agarose stamp. ${ }^{[31]}$ A PDMS stamp, for instance, is normally manufactured by polymerizing a siloxane mixture on a microfabricated silicon master as demonstrated in Figure 1.14 and directly used for printing.
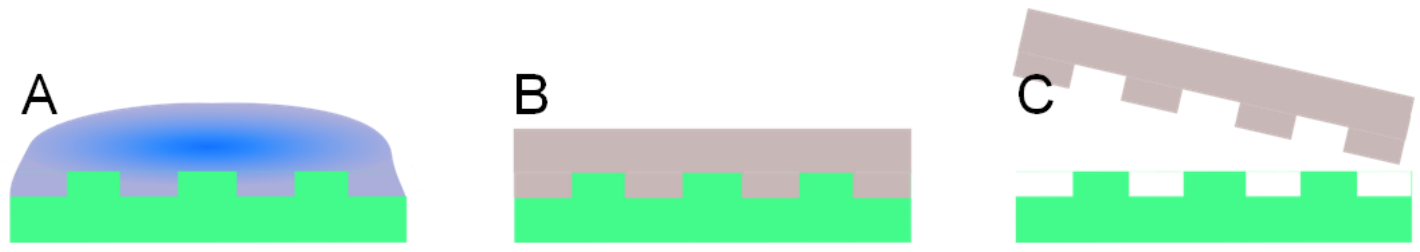

Figure 1.14: Preparation of a PDMS stamp with a silicon master. After microstructuring the silicon master by photolithography it is used as a negative form for curing the polymer on it. To produce a PDMS stamp a mixture of siloxanes is polymerized at elevated temperatures (A to B). The stamp can easily be peeled off the master after complete polymerization (C).

Stamped surfaces can be further modified after the stamping process. For example, silane solutions can be stamped on silicon or glass surfaces in order to perform chemical sensing or ligand binding in a second step. ${ }^{[32-34]}$ In addition to that, these surfaces can be used to spread lipid membranes. Lenhert developed a method called parallel dip-pen nanolithography allowing for a high-throughput patterning of surfaces with different kinds of lipid ink. ${ }^{[35]}$

\footnotetext{
${ }^{9}$ Adapted from 31. Falconnet, D., et al. (2006). "Surface engineering approaches to micropattern surfaces for cellbased assays." Biomaterials 27(16): 3044-3063 with permission of Elsevier.
} 
In contrast to the dip-pen technique, which allows printing structures with a minimum size of $30 \mathrm{~nm}$ and of a distance of $100 \mathrm{~nm}$, the $\mu \mathrm{CP}$ technique only reaches a size of $300 \mathrm{~nm}$.

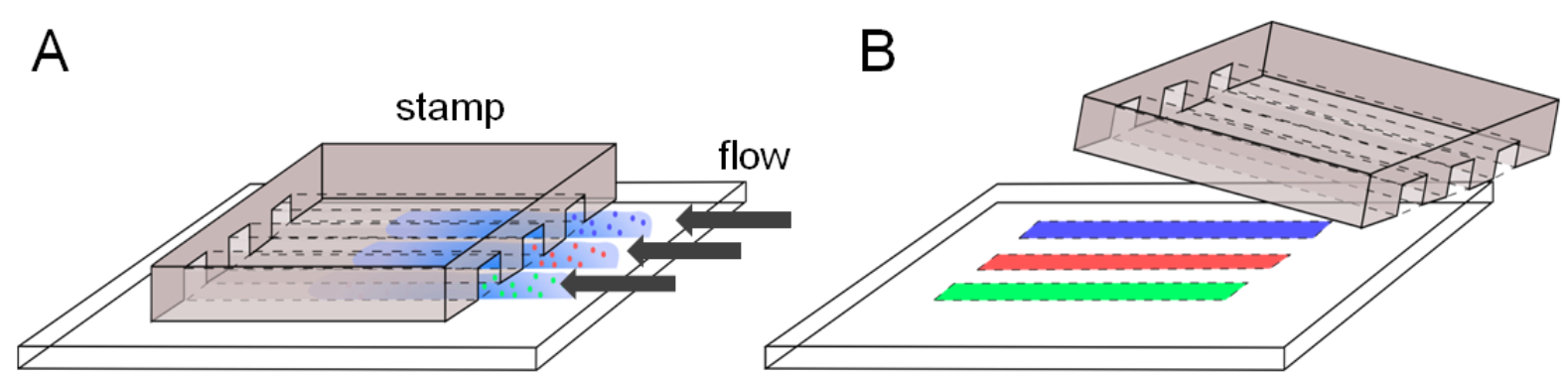

substrate

Figure 1.15: Microfluidic patterning ( $\mu$ FLP), also known as micromolding in capillaries (MIMIC).9 An elastomeric stamp is bound to the substrate to create micrometer-sized channels. The molecular ink flows through these channels due to capillary forces to give functionalized stripes on the surface (marked in red, blue and green). Each channel can be used for a different kind of ink allowing for the production of stripes with different binding abilities.

Using the vesicle spreading technique combined with PDMS molds exhibiting micrometersized channels can also produce micro-structured surfaces. This technique is known as microfluidic patterning $(\mu \mathrm{FLP} \text {, Figure } 1.15)^{[31,36]}$ or micromolding in capillaries (MIMIC) and gives access to microstructures with different functionalities on the same sample.

\subsection{Force spectroscopy measurements of biological interactions}

For the understanding of the nature of non-covalent forces acting between ligands and receptors in a lipid matrix, it is crucial to determine the forces acting between the surfaces. Dynamic loading of chemical bonds allows for the extraction of kinetic parameters, which can be used to explore the energy landscape of an interaction. Different techniques should be considered for the measurement of membrane-membrane interaction forces: the surface force apparatus, optical tweezers, micropipette aspiration technique and the atomic force microscope (Figure 1.16). ${ }^{[37]}$ With the surface force apparatus (SFA, Figure 1.16-A) forces acting between two mica cylinders can be measured with a vertical resolution in the Angstrøm regime. However, the force resolution is limited to $\sim 10 \mathrm{nN}^{[38]}$ In this setup, the cylinders are arranged perpendicular to each other and the forces acting on those are detected via multiple beam interferometry. The measurement is time-consuming and the setup does not allow for the use of different probe geometries or cell probes. 
Optical traps allow high-speed tracking of viruses as well as the measurement of forces down to $1 \mathrm{pN}$ and distance resolution of $\sim 5 \mathrm{~nm}$ (Figure 1.16-B). ${ }^{[37,39]}$ Despite the extremely high force sensitivity the setup is not suited for the analysis of multiple bond systems exhibiting forces up to several nanoNewton. In biomembrane force probe setups, a neutrophil or a red blood cell is used as a spring with known stiffness (Figure 1.16-D). It is trapped with a micropipette and is used to determine the forces acting between a functionalized bead and a leukocyte.
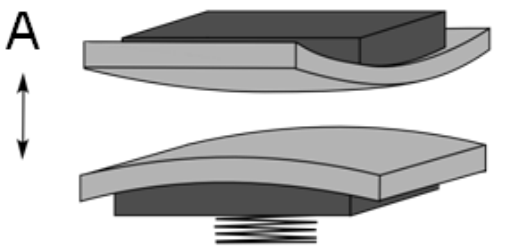

$10 \mathrm{nN}-1 \mu \mathrm{N}$
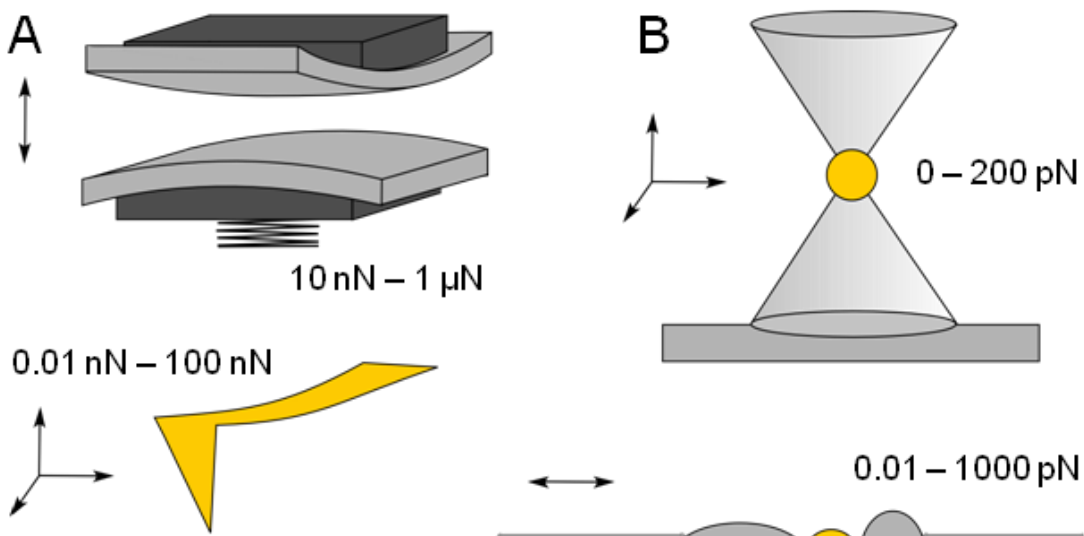

$\mathrm{N}-100 \mathrm{nN}$
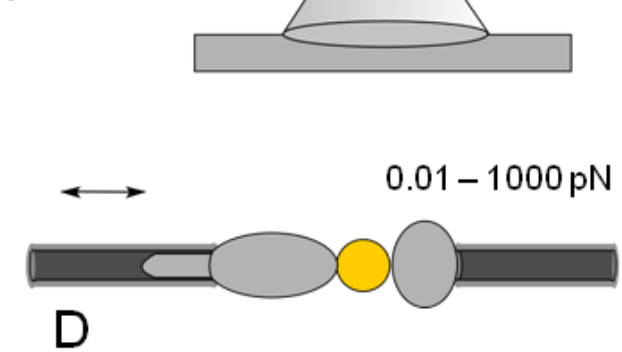

Figure 1.16: Techniques to probe adhesion phenomena. ${ }^{10}$ The surface force apparatus uses two micacoated cylinders perpendicular to each other and reaches a force resolution of $10 \mathrm{nN}$ to $1 \mu \mathrm{N}$ (A). An optical tweezers setup as depicted in (B) allows measuring forces of $1 \mathrm{pN}$ to $200 \mathrm{pN}$. The atomic force microscope shown in (C) reaches forces of several tens of nanoNewton with a force resolution of $10 \mathrm{pN}$. With the micropipette aspiration technique (D) forces between $0.01 \mathrm{pN}$ and $1 \mathrm{nN}$ are accessible (shown in a biomembrane force probe setup).

Besides topographical imaging, the atomic force microscope (AFM) also allows for force spectroscopic measurements on the sample surface (Figure 1.16-C). In such a setup, the tip of a commercial cantilever is functionalized with a specific molecule and used to probe structures on the sample surface. In the last decades, single molecule force spectroscopy with an AFM setup (Figure 1.17) has reached tremendous importance when it comes to the analysis of kinetics of chemical bond formation and the mechanics of the bonds. With a vertical resolution of $1 \mathrm{~nm}$ and a force resolution down to $10 \mathrm{pN}$, folding and unfolding processes of proteins as well individual hydrogen bonds are accessible.

\footnotetext{
${ }^{10}$ Adapted from 37. Leckband, D. (2000). "MEASURING THE FORCES THAT CONTROL PROTEIN INTERACTIONS." Annual Review of Biophysics and Biomolecular Structure 29(1): 1-26.
} 
Surface interactions due to electrostatic forces, Van-der-Waals forces, hydrogen bonds, hydrophobic and ionic interactions, as well as covalent bonds can be analyzed with this technique. Surfaces are usually functionalized by using soft lithography methods as described in chapter 1.5 .
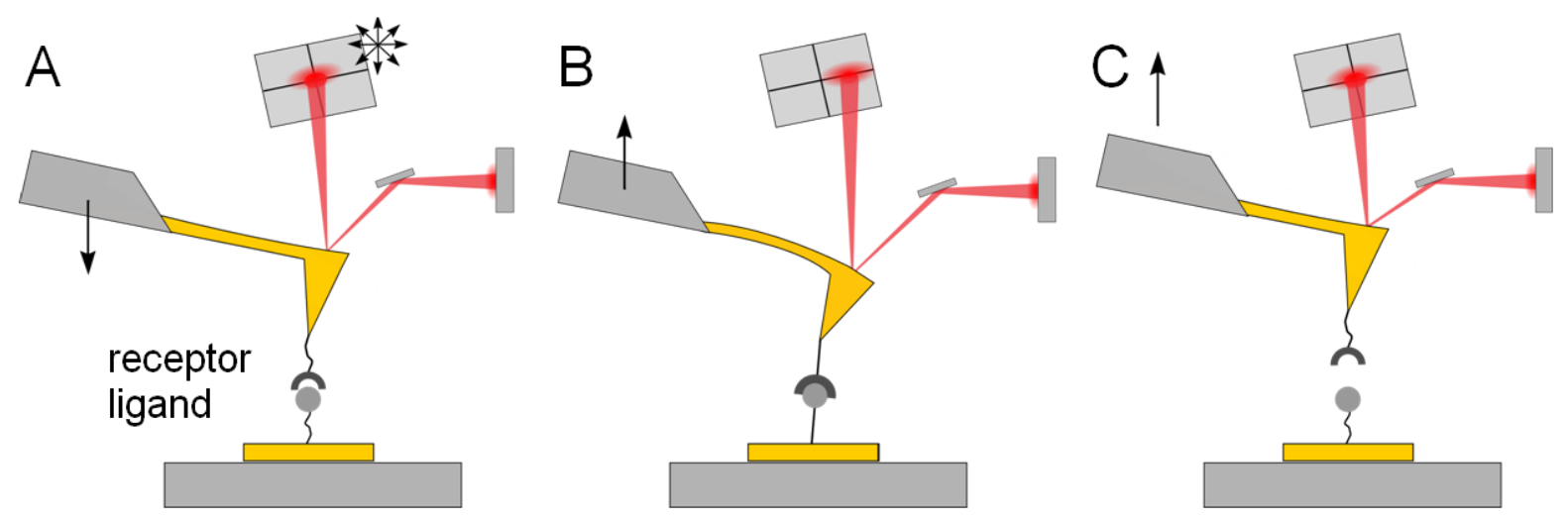

Figure 1.17: Single molecule force spectroscopy setup probing a receptor-ligand interaction. Upon interaction between a cantilever functionalized with receptor molecules and a flat support functionalized with ligand molecules the cantilever bends towards the surface while it is moved away from the surface. The force resolution limit of this method is $\sim 10 \mathrm{pN}$ with a maximum of detectable forces in the $\mathrm{nN}$ regime.

In terms of force resolution, the AFM technique is dominated by the optical tweezers setup but in contrast to the optical tweezers setup, the AFM setup gives also access to the $\mathrm{nN}$ regime, which is vital for the investigation of cellular interaction processes.

Both techniques allow for the application of force ramps to chemical bonds, which can be used to determine energy potentials of the system.

\subsubsection{Force spectroscopy of cell-to-substrate-adhesion}

If the single cell probe technique introduced in chapter 1.6 is performed on a functionalized substrate, different states of the cellular adhesion process can be objected, for instance the cell spreading behavior on different substrate stiffness or topography. This is of particular interest for the construction of medical equipment, which comes into contact with cell suspensions, i.e. implants or transfusion plastics. Integrin-RGD interactions can be probed in order to analyze focal contact dynamics as well as the overall adhesion strength of a cell. 


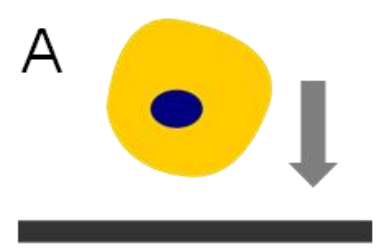

$\mathrm{B}$
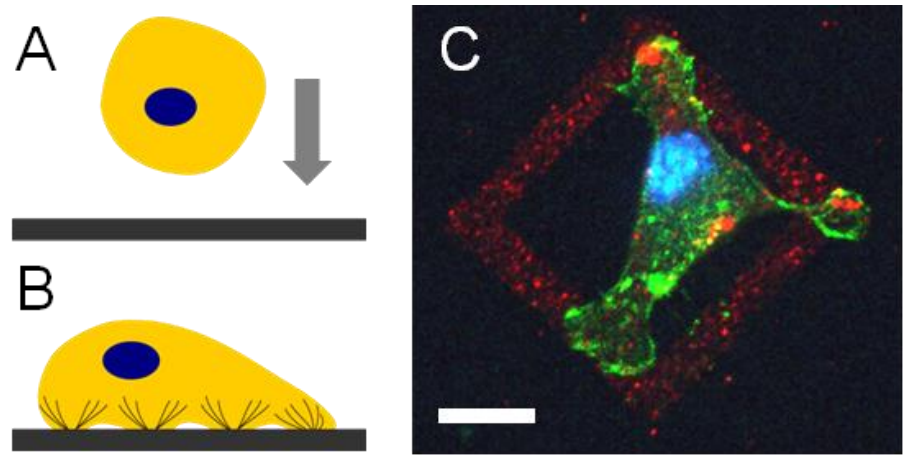

Figure 1.18: Cellular adhesion (A to $B$ ) requires functionalization of the surface. C: A single HEK cell adhering to a pattern functionalized with cadherin-constructs. Scale bar: $10 \mu \mathrm{m} .^{11}$

Figure 1.18 demonstrates how a cell controls its adhesion via focal contacts. Actin stress fibers dynamics largely depends on the forces generated by number and distance of focal contact areas. ${ }^{[40]}$ Spreading mechanism can be probed with AFM techniques involving native cells such as the single-cell force spectroscopy or the cell monolayer spectroscopy. ${ }^{[42,43]}$
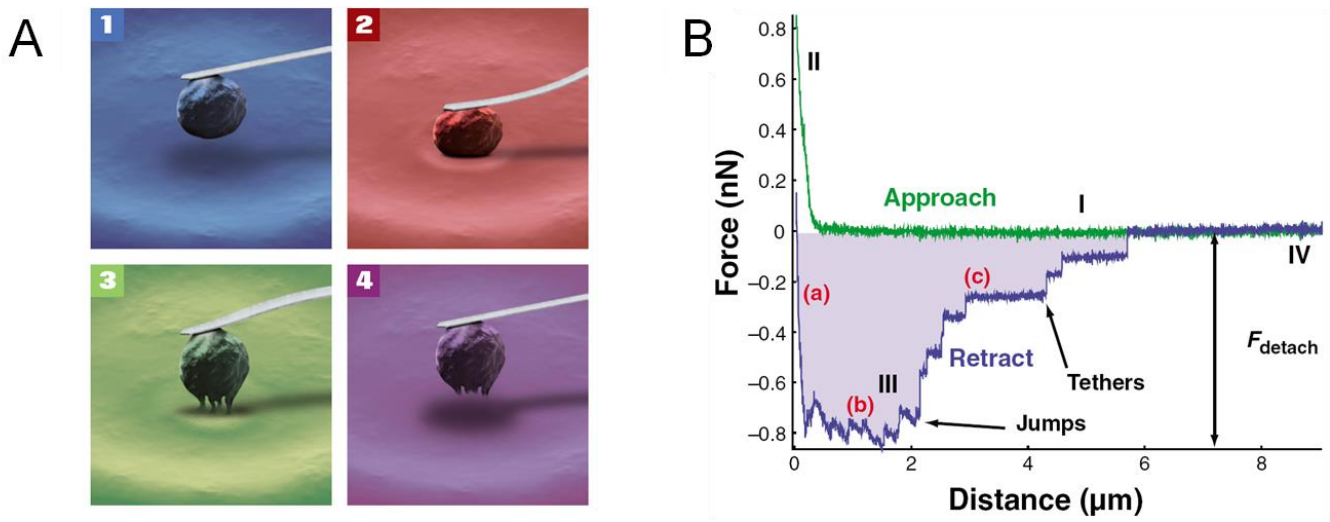

Figure 1.19: Cell-substrate interaction probed by single cell force spectroscopy. ${ }^{12}$ Experimental setup of the single cell force spectroscopy technique on a hard substrate $(A)$ and profile acquired while contacting the surface with the cell probe (B). ${ }^{[41]}$ After a short contact time, the cantilever is retracted and the cell performs an adhesion process resulting in the given profile. Detachment force, receptor unbinding forces (jumps), occurrence of tethers as well as work of detachment (shaded area) has been detected by Friedrichs et al.

Force profiles (or force (-distance) curves) resulting from a single-cell force-distance curve (setup shown in Figure 1.20) are shown in Figure 1.19. The retraction trace of the forcedistance curve shows rupture events in close distance to the surface as well as extended plateaus at constant force indicative for the formation of membrane tethers between cell and substrate.

\footnotetext{
${ }^{11}$ Image provided by Dagmar Fichtner, Karlsruhe, and used with her permission.

${ }^{12}$ Images kindly provided by JPK Instruments AG, Bouchéstr 12, 12435 Berlin, Cellhesion brochure/website
} 


\subsubsection{Force spectroscopy of cell-cell interactions}

Cell-cell interactions can be studied in a force spectroscopy setup as depicted in Figure 1.20. Leckband et al. subjected intercellular adhesion in order to extract information about intermembrane adhesion. ${ }^{[44]}$

A recently established setup implies a soft cantilever, which is equipped with a native cell to probe a functionalized surface (Chapter 1.6) or a second cell (Figure 1.20). ${ }^{[42]}$
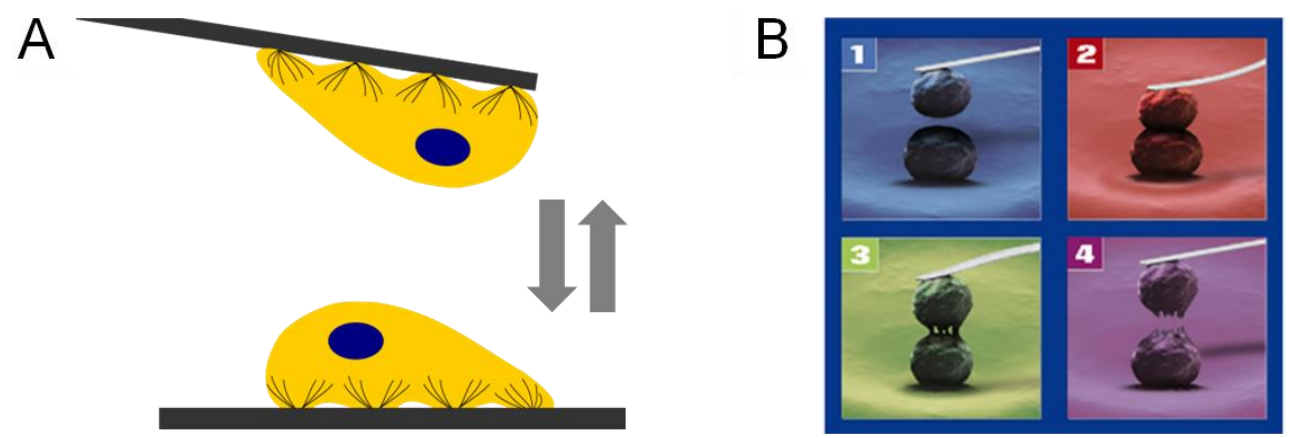

Figure 1.20: Probing cell-cell interactions by using the single-cell force spectroscopy technique on an adherent cell. By retracting the cantilever interaction forces as well as tether formation processes are accessible (B). ${ }^{12}$

Cell-to-cell adhesion and tether formation processes are probed by retracting the cantilever after a short cell contact time. Single cell force spectroscopy allows for the determination of large contact interactions of CAMs between various kinds of cells and serves as a valuable supplement for the single molecule force spectroscopy technique. ${ }^{[45,46]}$

\subsubsection{Force spectroscopy of membrane tethers}

Due to the viscoelasticity of membrane tethers, cells are able to overcome distances of several tens of micrometers as demonstrated by Schmitz et al and others. ${ }^{[47-50]}$ Besides, microtubule-based membrane extensions, which are used for cell locomotion, membrane tethers are built between cells in order to enable transcellular transport processes. Tethers are liquidfilled tubes consisting of a cylindrical lipid bilayer connecting two large membrane compartments with each other (Figure 1.21-A and B). From micropipette suction experiments Waugh, Hochmuth, and Evans were able to determine mechanical membrane properties such as inplane tension, bending modulus, membrane viscosity, and inter-bilayer shear. ${ }^{[53-55]}$ 

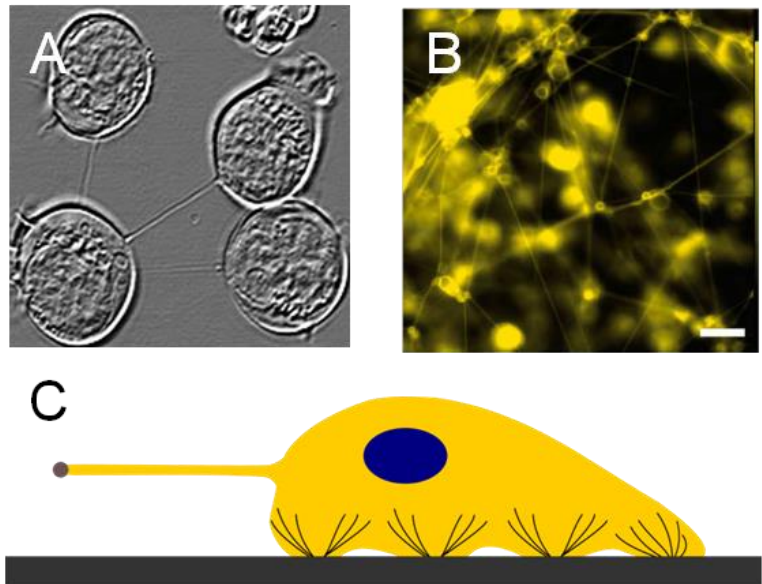

Figure 1.21: Pulling lipid tethers. A: Membrane tethers between human T cells. ${ }^{13}$ B: Network of tethers between artificial DOPC/GM3 giant unilamellar vesicles. ${ }^{[51], 14} \mathrm{C}$ : Lipid tethers can be pulled from adherent cells by using magnetic particles, which are optically trapped or magnetic. ${ }^{[52]}$ Lipid tethers are characterized by constant force plateaus in force-distance curves such as shown in Figure 1.19.

Sheetz and coworkers pulled tethers from neuronal growth cone membranes through the use of IgG-coated beads in an optical tweezers setup (Figure 1.21-C). ${ }^{[52,56]}$ They found that the force of the tether on the bead was higher than forces generated by single molecular motors such as kinesin and myosin and that the force increased linearly with the velocity of tether elongation. ${ }^{[52]}$ Furthermore, the apparent membrane viscosity was estimated and it was stated that membrane-cytoskeleton interactions influence the probability of tether formation as well as the critical force required to pull a tether. In conjunction with that, Sun et al. found decreasing tether rupture forces upon disruption of actin cytoskeleton and removal of glycocalyx compounds when they pulled multiple membrane tethers from adherent cells, Sun employed poly-L-lysine. ${ }^{[57]}$ Tether formation is also observed in cell-substrate or in cell-cell spectroscopy, where strong coupling of surface structures induces a flux of membrane material. ${ }^{[58]}$ Müller and coworkers dynamically probed tethers from mesendoderm zebrafish embryo cells by employing Concanavalin A-modified AFM tips in order to exert a constant force on the bond formed between lectin and cell surface oligosaccharides. From those measurements, they extracted kinetic parameters (bond lifetime/ off rates, potential width) and found a non-linear relationship between tether forces and pulling velocity. These findings are attributed to the non-ideality of pulled membranes, which were composed of lipids and proteins.

\footnotetext{
${ }^{13}$ Image kindly provided by Dan Davis, Dan Davis Lab, London.

${ }^{14}$ Image reprinted with permission of Elsevier.
} 


\subsubsection{Force spectroscopy of fusion processes}

Among the considerable spectrum of processes involving membrane-membrane interactions, the understanding of cell-cell contacts and membrane fusion is of particular interest. Membrane fusion occurs when two separate lipid membranes merge into a single continuous bilayer and plays a crucial role in embryogenesis, neurophysiology as well as viral infection. Regardless of the process, the sequence of events, which is driven by subtle changes in free energy requires almost without exception an initial specific recognition that triggers the subsequent dynamic response of the bilayer. This specific recognition between ligands on one membrane and the receptor displayed by the corresponding counterpart produces the initial molecular connection between two membranes, which eventually determines the fate of the membrane-membrane assembly, i.e. aggregation, hemifusion or full fusion. The exact mechanism of membrane fusion is still a matter of debate. However, three stages in the fusion pathway are reported, contact (Figure 1.22-A and B), hemifusion ( $C$ and $D)$, and the opening of an expanding fusion pore as depicted in Figure 1.22- E and F, respectively.

A Initial contact

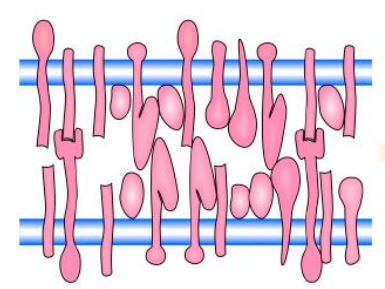

C Hemifusion stalk

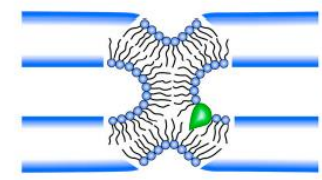

B Contact between protein- $F$ depleted bilayer patches

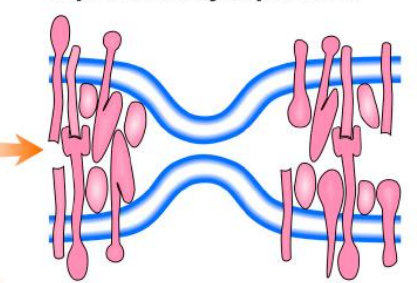

D Hemifusion diaphragm

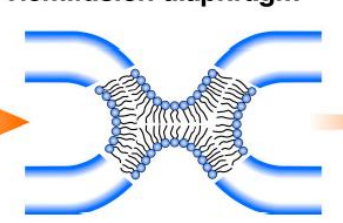

Post-fusion conformation

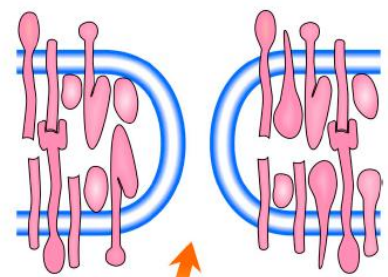

E

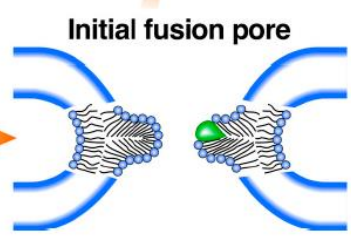

Figure 1.22: Membrane fusion through hemifusion intermediates. ${ }^{[59], 15}$ Fusion equals the merging process of two separate lipid membranes leading to the unification of the compartments, which have been restricted by the membranes before. If membranes are separated by a critical distance (A to B), a hemifusion stalk (C) is formed, which is characterized by the fusion of proximal membrane leaflets. Extension of the stalk into a hemifusion diaphragm (D) is followed by the formation of a fusion pore (E), which implies the successful fusion of distal leaflets. The post-fusion conformation depicted in $F$ is characterized by a connection of previously separated liquid compartments.

\footnotetext{
${ }^{15}$ Image reprinted from [59] as non-commercial third party reuse, Rockefeller University Press.
} 
It is a process that requires membrane deformation and merging of two adjacent lipid bilayers. When bilayers are separated below $1 \mathrm{~nm}$, the energy of repulsion due to the pervasion of the hydration shell is assumed to drive fusion. It is widely accepted that formation of a fusion stalk and its expansion into a hemifusion diaphragm relaxes the hydration energy and hence provides the necessary driving force. Forces and, as a consequence, distances between two membranes can either be tuned by changing the interaction potential using charged lipids or varying the electrolyte or more directly using force probes such as optical/magnetic tweezers, the atomic force microscope or the surface force apparatus. Israelachvili and coworkers could assign hemifusion and full fusion events of two DMPC bilayers (dimyristoylphosphocholine, two saturated $\mathrm{C}_{14}$ chains) immobilized on polyetherimide/mica to instabilities in force distances curves. Full fusion was reported to occur only at very high joining pressure.

Besides fusogenic molecules, parameters such as spontaneous curvature of membranes induced by lipid geometries, $\mathrm{Ca}^{2+}$ content of the solution, and $\mathrm{pH}$ are known to facilitate or induce fusion. ${ }^{[59-61]}$

Abdulreda et al used a membrane probe setup to determine membrane interactions mediated by proteins of the SNARE (Soluble $\mathrm{N}$-ethylmaleimide-sensitive factor attachment protein receptor) complex. ${ }^{[62]}$ Due to two consecutive instabilities observed in the approach curve the authors interpret their results in terms of SNARE mediated full fusion. Israelachvili and coworkers could assign hemifusion and full fusion events of two DMPC bilayers immobilized on PEI (polyetherimide)/mica to instabilities in force distances curves. ${ }^{[63,64]}$ Full fusion was reported to occur only at very high joining pressure. 


\subsection{Motivation}

The mechanics and dynamics of plasma membranes play a crucial role in many cellular events such as adhesion, motility, membrane fusion, as well as exo- and endocytosis of mammalian cells. Particularly, membrane-membrane interactions display great versatility since molecular recognition, deformation, adhesion, pore formation, and fusion of lipid bilayers might occur depending on an intricate interplay between proteins, lipids, and forces. We are interested in the overall understanding of cellular interaction mechanisms, which requires suitable model systems that allow the investigation of single aspects of the processes mentioned above. Different top-down and bottom-up approaches have been presented up to now.

However, since bulk techniques operate an ensemble averaging, they are not able to elucidate a variety of aspects inherent to individual molecules, e.g. rare events, transient phenomena, crowding effects, population heterogeneity, etc. ${ }^{[65]}$ So far, a bulk measurement of ligandreceptor-interactions in a cell-like setup allowing for the accurate dosage of contactmediating molecules has not been established. Of special interest in such a setup is the access to thermodynamic, kinetic and mechanical parameters from bond clusters to single bonds.

The drawback of conventional sharp tips for functionalization with lipid bilayers is their high curvature and therefore the difficulty to accomplish a successful functionalization. Particularly, the often undefined geometry of the tip renders conventional AFM cantilever unsuitable to quantify membrane-membrane interactions. In contrast, colloidal probe microscopy combines the merits of a defined probe geometry and controllable surface functionalization with the potentiality to conduct site-specific analysis of force distance curves. ${ }^{[66]}$

Our idea was to use the defined geometry of a colloidal probe to mimic cellular interactions such as cell-substrate, cell-cell, and cell-virus interactions by bringing artificial lipid membranes in contact to probe cell-cell, cell-substrate, and cell-virus contacts. In contrast to single molecule force spectroscopy techniques, we envision to enclose the measurement of lateral diffusion and the formation of bond clusters to be additional features of our system. Particularly, we seek to capture the impact of multiple bonds in the contact zone on the dynamic strength of the system under a force ramp. 
Compared to top-down setups using native cells the advantage of the membrane probe spectroscopy setup is to specifically tune artificial membranes to provide the functionality required for each specific kind of interaction.
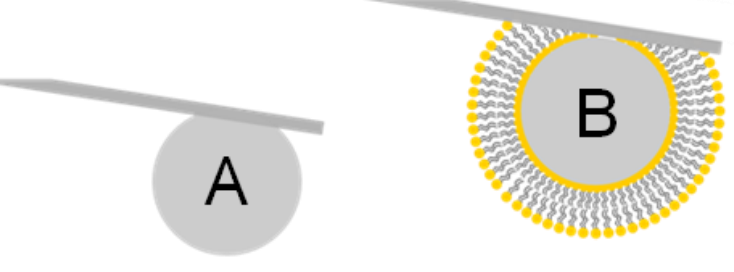

mummummmmmmm

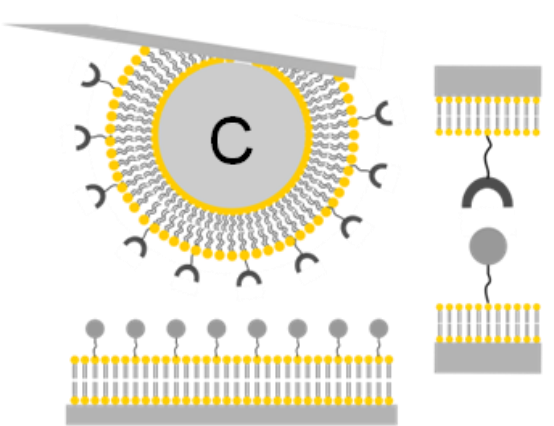

Figure 1.23: Concept of membrane probe spectroscopy, which is using the colloidal probe microscopy setup in combination with solid-supported lipid bilayers for the study of cellular adhesion, repelling properties of the cell surface, (inhibited) fusion, and membrane tethering.

Figure 1.23 depicts the general setup of the membrane probe spectroscopy setup. By including functionalized lipid molecules into the membranes we are able to couple a large variety of ligand and receptor molecules to the surfaces. The lipid composition can be adjusted in order to modify the thermodynamic properties of the membranes.

The system allows for the investigation of homomeric and heteromeric setups in nearphysiological conditions and gives access to the detection of adhesion and subsequent fusion phenomena. Most important topics addressed in this work are the consequences of geometrically confined areas for the overall dynamic adhesion strength, the impact of stochastic nature of individual bonds and external forces on cluster size, the influence of membrane fluidity on the adhesion strength, and the question how important elastic properties of the cell and the substrate are for function of adhesion force. 


\section{Model systems}

\subsection{Strong interactions: $\mathrm{Ni}^{2+}-\mathrm{NTA}-\mathrm{H} 6$}

Known from affinity chromatography purification of proteins the coordinative interaction between $\mathrm{Ni}^{2+}$ nitrilotriacetate $\left(\mathrm{Ni}^{2+}-\mathrm{NTA}\right)$ and histidine (His) residues is almost as strong as a covalent interaction. ${ }^{[67,68]}$

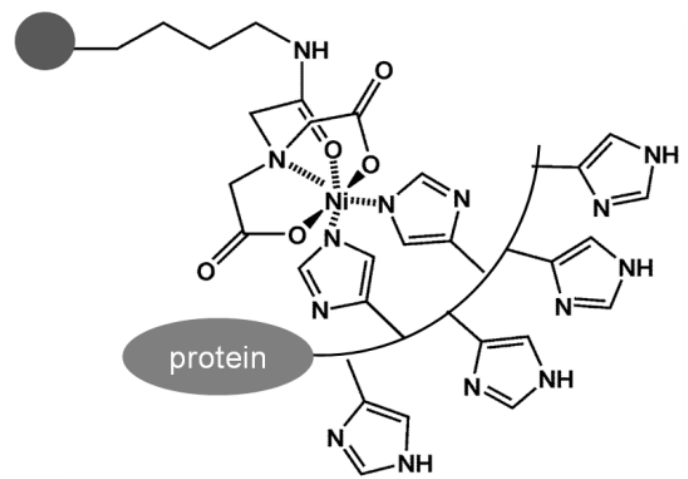

Figure 2.1: $\mathrm{Ni}^{2+}-\mathrm{NTA}$-Histag interaction for the purification of His-tagged proteins via $\mathrm{Ni}^{2+}-\mathrm{NTA}$ agarose beads. ${ }^{16}$ Each $\mathrm{Ni}^{2+}-\mathrm{NTA}$ moiety binds two out of six histidine residues of the $\mathrm{H} 6$ unit and can be eluted by the usage of imidazole.

The two free binding sites of the hexagonally arranged $\mathrm{Ni}^{2+}-\mathrm{NTA}$ chelator serve as receptors for oligo-histidines as depicted in (Figure 2.1). Schmitt developed $\mathrm{Ni}^{2+}$-NTA-functionalized lipids as well as $\mathrm{Ni}^{2+}$-NTA-tagged thiols in order to functionalize membranes and gold surfaces, respectively. ${ }^{[69,70]}$ Lauer and Nolan used $\mathrm{Ni}^{2+}-\mathrm{NTA}$ bearing microspheres with or without membrane. ${ }^{[72]}$ Verbelen et al. used $\mathrm{Ni}^{2+}-\mathrm{NTA}$ thiols to deposit short His-tagged peptide sequences on AFM tips with the aim to perform single molecule force spectroscopy measurements (Figure 2.2). ${ }^{[71,73]}$ They found three maxima in the $\mathrm{Ni}^{2+}$-His rupture force histogram, which they attribute to monovalent and multivalent interactions between a single Histag and one, two, or three NTA groups. As the forces ranging from 150 to $470 \mathrm{pN}$ are higher than interaction forces of many biologically relevant interactions, they consider this setup to be a well-suited platform for the stable immobilization of proteins for AFM studies. A combination of these two methods is the technique developed by Liu et al. ${ }^{[74]}$

\footnotetext{
${ }^{16}$ Adapted from www.promega.com, used with permission of Jan Lundsted, promega.
} 


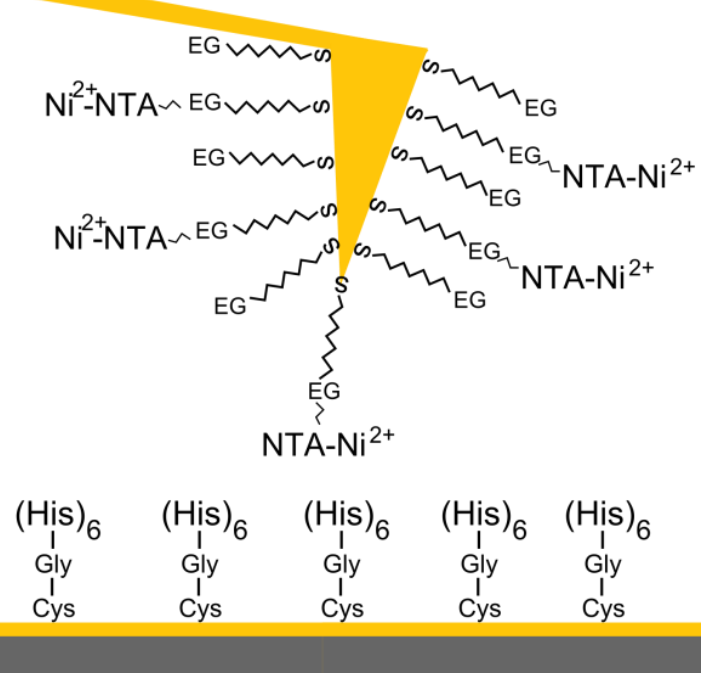

Figure 2.2: Single molecule force spectroscopy setup using functionalized gold surfaces to probe the $\mathrm{Ni}^{2+}$ NTA His-tag interaction. ${ }^{17}$ Verbelen et al. used ethylene glycol (EG) thiols equipped with $\mathrm{Ni}^{2+}$-NTA-EG thiols on the AFM tip acting as receptors for short his-tagged peptides on a planar substrate. ${ }^{[71]}$

They used the coordination concept to immobilized microspheres on AFM tips and reported the His-tag- $\mathrm{Ni}^{2+}$ bead force to be strong enough to bind microspheres for the spectroscopy of SNARE proteins, which were coupled to the microspheres. Moreover, cantilevers can directly be coated with a thin Nickel layer in order to immobilize his-tagged molecules without additional thiol layer.[74]
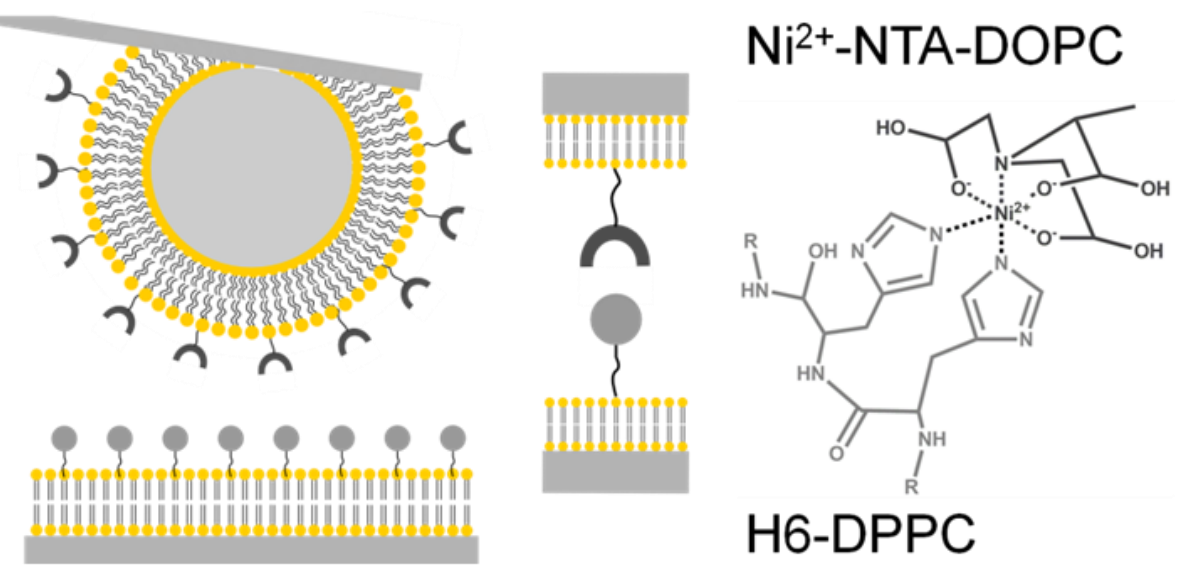

Figure 2.3: Schematic of the heteromeric membrane probe spectroscopy setup as used for the detection of the $\mathrm{Ni}^{2+}$-NTA-His-tag interaction. ${ }^{[75], 18}$ Functionalization of the colloidal probe with $\mathrm{Ni}^{2+}$-NTA moieties (dark grey crescent symbols) is achieved by spreading small unilamellar vesicles, which contain a certain percentage of $\mathrm{Ni}^{2+}$-NTA bearing phospholipids. The corresponding membrane on the planar support is decorated with short His-tagged peptides Ac-H6GG-NH (“H6") (grey circles).

\footnotetext{
${ }^{17}$ Adapted from 71. Verbelen, C., et al. (2007). "The NTA-His6 bond is strong enough for AFM single-molecular recognition studies." Journal of Molecular Recognition 20(6): 490-494.

${ }^{18}$ Image reprinted from [75] with permission of Elsevier.
} 
We introduce a membrane probe spectroscopy setup used to measure membranemembrane interaction processes functionalized with receptors and ligands, respectively. A colloidal probe cantilever is coated with a $\mathrm{Ni}^{2+}{ }_{-}$NTA bearing membrane, whereas a membrane on a solid support is functionalized with His-tagged peptides (Figure 2.3).

We chose $\mathrm{Ni}^{2+}$-NTA-labeled lipids in order to dope our solid-supported lipid bilayers with receptor molecules that serve to probe the strength of the coordinative interaction between $\mathrm{Ni}^{2+}$ ions and histidine residues.

\subsection{Weak interactions: Disaccharides derived from Microciona prolifera}

Apart from strong non-covalent interactions weak interactions are considerably more important since clustering in lateral orientation provides strength by numbers and organization. Working with weak interactions gives you the freedom of precisely adjusting the strength of interaction subjected to being probed as a broad range of forces can be covered from the single-molecule force level to large cluster forces involving monovalent or multivalent structures. $^{[76]}$
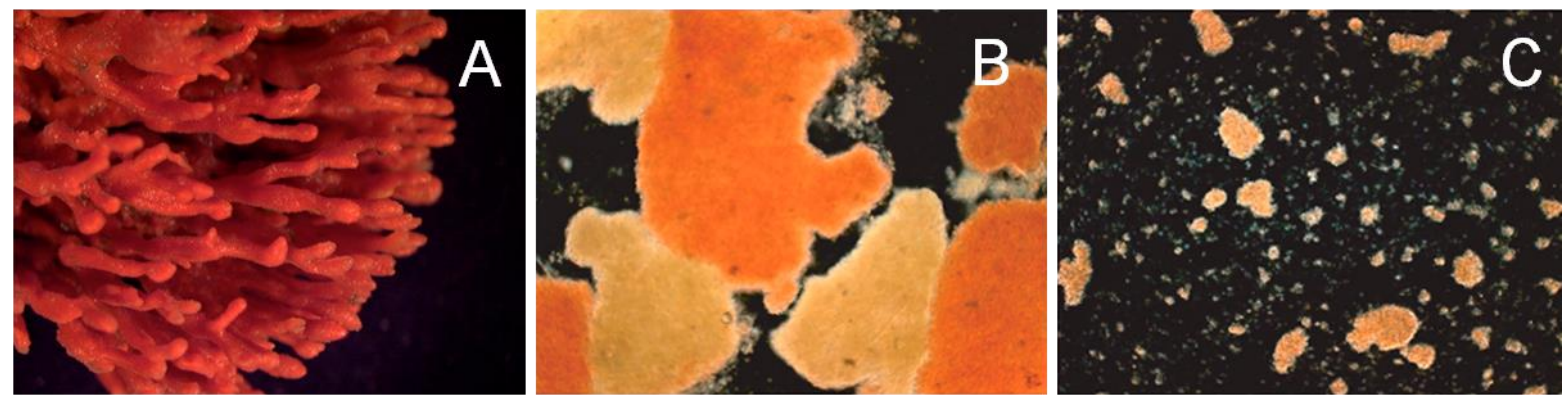

Figure 2.4: The red beard sponge Microciona prolifera and the $\mathrm{Ca}^{2+}$-dependent self-recognition. A: The red beard sponge as found at the US Atlantic coast from Prince Edward Island to Texas and as registered by Ellis \& Solander in $1786 .{ }^{19,[77]}$ B: Microciona prolifera (orange) and Suberites fuscus (yellow) cells in $10 \mathrm{mM} \mathrm{Ca}^{2+}$ solution build separate colonies due to specific recognition of their glycosylation patterns. ${ }^{[78], 20} \mathrm{C}$ : The interaction of Microciona prolifera is disturbed after depletion of $\mathrm{Ca}^{2+}-$ ions. ${ }^{[78], 20}$

\footnotetext{
${ }^{19}$ Image taken from the U.S. Geological Survey

${ }^{20}$ Image reprinted from [78] ] as non-commercial third party reuse, Rockefeller University Press.
} 
Typical cellular model systems employing weak carbohydrate-carbohydrate interactions are sponge cells possessing glycan epitopes on their surfaces. These glycans have been proven to have a significant impact on mammalian cell adhesion processes. ${ }^{[79-82]}$ The cells of the red beard sponge Microciona prolifera (

Figure 2.4-A) possess sulfated disaccharide structures, which are mediating self-recognition (

Figure 2.4-B) as well as an inactive sulfated galactose-linked tetrasaccharide. ${ }^{[77,83]}$
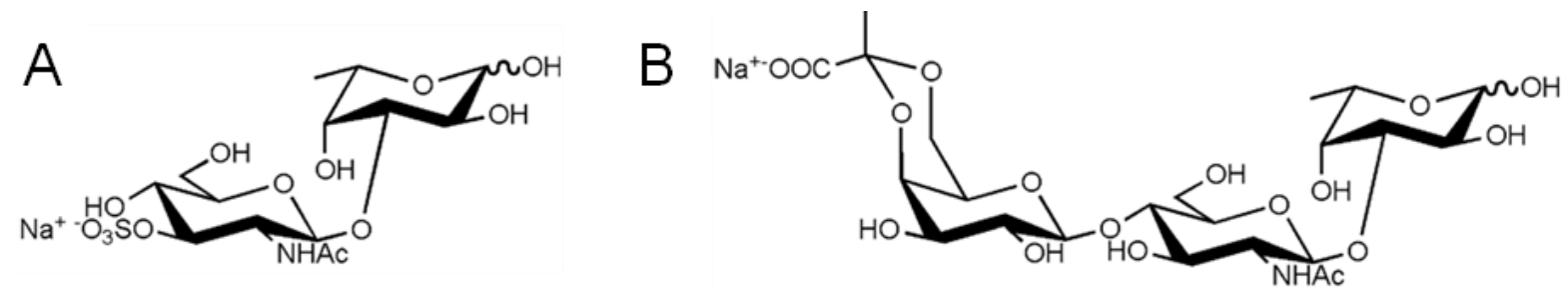

Figure 2.5: Microciona prolifera self-recognition units: sulfated disaccharide (A) and pyruvated trisaccharide (B). ${ }^{[85], 21}$ The structures involved in our measurements are derived from the sulfated disaccharide depicted in $\mathbf{A}$.

The sulfated epitope is responsible for the $\mathrm{Ca}^{2+}$-dependent (

Figure 2.4-C) self-recognition processes between Microciona prolifera cells. ${ }^{[84]}$ The key structures of this process in cells of the marine sponge Microciona prolifera are the pyruvated tri-

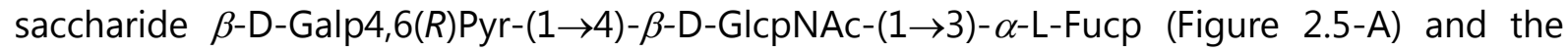
sulfated disaccharide ( $\beta$-D-GlcpNAc3S-(1 $\rightarrow 3)-\alpha$-L: -Fucp, Figure $2.5-\mathrm{B})$.

Sulfated disaccharides are presented on the g-200 unit of the Microciona prolifera aggregation factor (MAF, Figure 2.6). In contrast to the calcium-independent $g-6$ unit, the $g-200$ is a $\mathrm{Ca}^{2+}$ dependent proteoglycan self-interaction domain, which forms a sunburst-like shape and mediates cellular recognition between sponge cells (Figure 2.6-D) of the same kind. ${ }^{[84,86,87]}$ The phenomenon of self-recognition was demonstrated by De Souza et al. who used particles functionalized with synthetic sulfated disaccharide moieties, which triggered particle aggregation on the seawater calcium concentration $\left(10 \mathrm{mM}\right.$, (Figure 2.7-A/B)). ${ }^{[89,90]}$ Bovine serum albumin (BSA)-disaccharide conjugates have also been used to prove the aggregation behavior by using surface plasmon resonance (SPR). ${ }^{[91]}{ }^{[87]}$ Anselmetti and co-workers were able to measure the self-recognition of $\mathrm{g}-200$ as a function of $\mathrm{Ca}^{2+}$ in solution using single-molecule force spectroscopy.

\footnotetext{
${ }^{21}$ Image reprinted from [85] with the permission of Springer.
} 

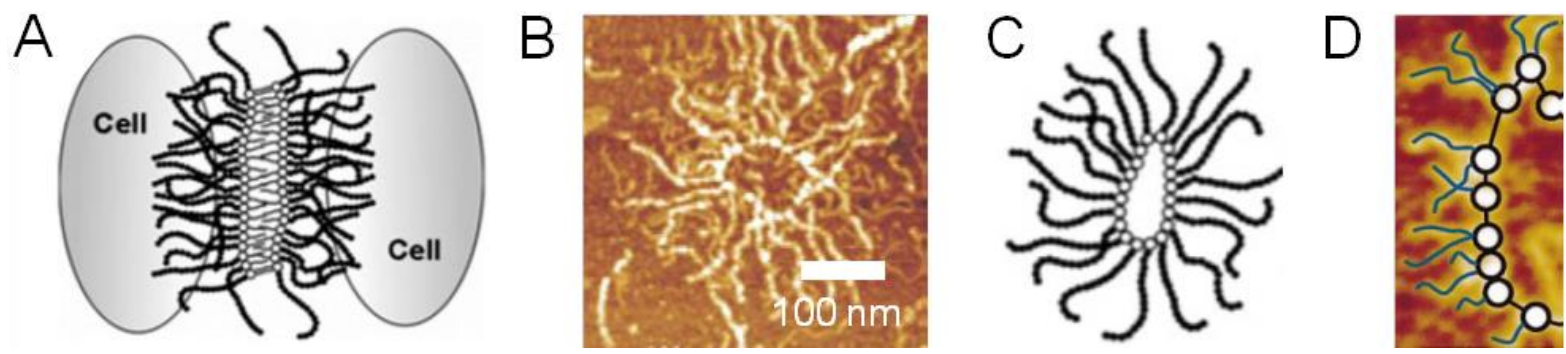

Figure 2.6: The Microciona prolifera aggregation factor (MAF) as reported by Garcia-Manyes and others. ${ }^{[84,}$ ${ }^{86-88]} A^{[87], 22}, B^{[86], 21}:$ AFM images of MAFs. The sunburst-like MAFp4 molecules possess about 50 copies of a $6 \mathrm{kDa}$ glycan (g-6) and act as cell-surface binding receptors ("arms"), the $200 \mathrm{kDa}$ acidic glycans (g-200, empty circles in $B, C^{[88], 23}, D^{[86], 21}$ ) of MAFp3 mediate calcium-dependent self-association of Microciona prolifera cells (D).

Binding forces between sulfated disaccharide epitopes were determined by performing AFM studies with functionalized gold surfaces to give multiples of $(30+/-) 6 \mathrm{pN}$ in the presence of calcium (Figure 2.7-C). ${ }^{[85]}$ The magnitude of adhesion forces was highly dependent on the divalent ion being present during the measurement. Magnesium ions seem to promote the interaction less effectively while calcium ions show strongest interaction.
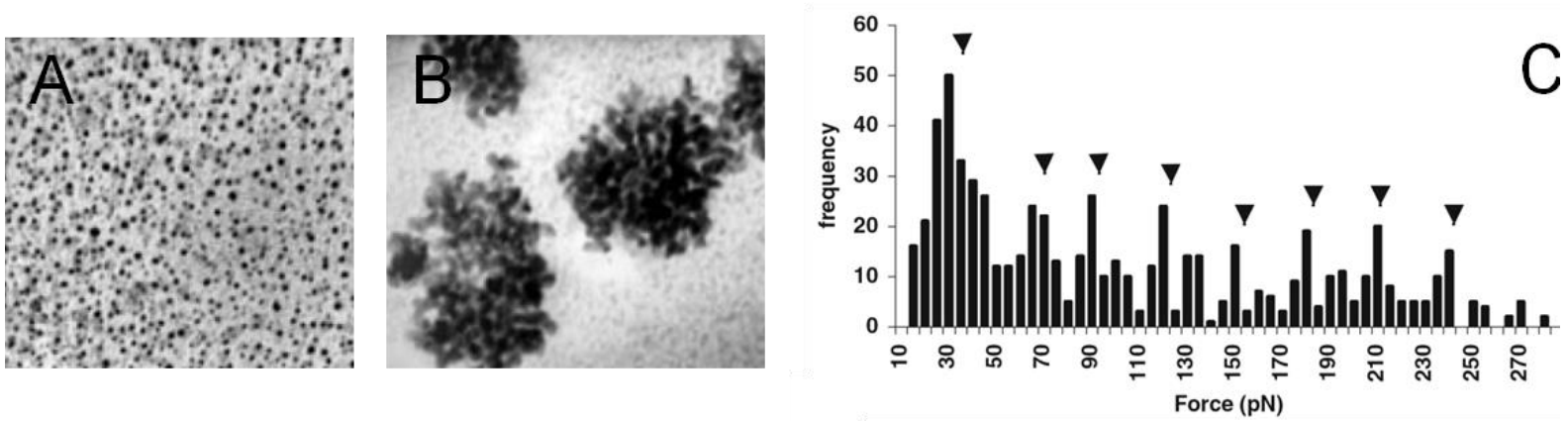

Figure 2.7: Proteoglycan self-recognition as reported by De Souza et al. ${ }^{[85,89], 24}$ TEM imaging shows that gold glyconanoparticles coated with the synthetic disaccharide epitope $\beta$-D-GlcpNAc3S-(1 $\rightarrow 3)$ - $\alpha$-L-Fucp can mimic the proteoglycan self-recognition that is responsible for marine sponge cell adhesion in a calcium-dependent manner. Particle aggregation only takes place in the presence of calcium ions (B). Any structural changes in the disaccharide completely eradicated the self-recognition phenomenon. A similar phenomenon was found in force spectroscopy measurements of the same interaction (C).

Based on an idea of the group by the group of Prof. K. Kumar in Boston, we performed force spectroscopy measurements with the membrane probe setup illustrated in Figure 2.8. In collaboration with the group of Dr. D. B. Werz, we functionalized membranes with sulfated and

\footnotetext{
${ }^{22}$ Image reprinted from [87] with the permission of Oxford University Press.

${ }^{23}$ Image reprinted from [88], no permission needed

${ }^{24}$ Images reprinted from [85] with the permission of Springer.
} 
non-sulfated disaccharides in order to determine the necessity of the sulfato group for specific recognition. ${ }^{[93]}$
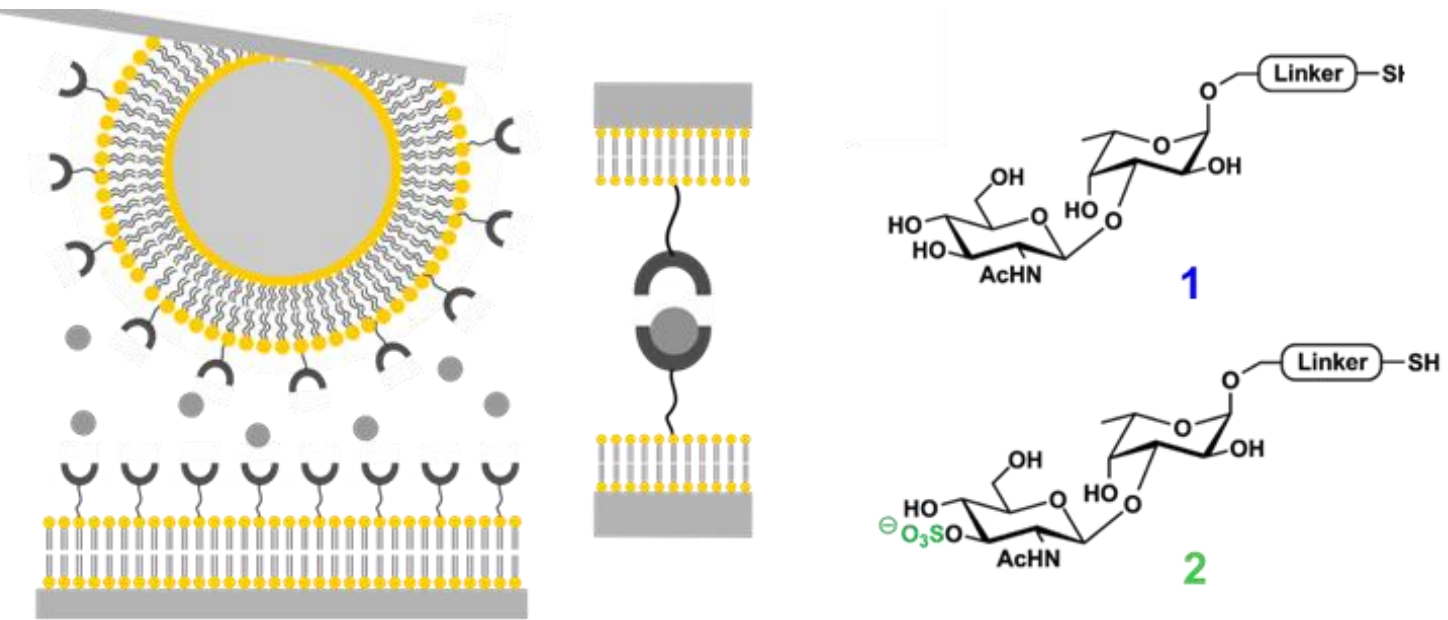

Figure 2.8: Schematic of homomeric membrane probe spectroscopy setup designed to study the homomeric $\mathrm{Ca}^{2+}$-dependent interaction between sulfated Microciona prolifera epitopes. ${ }^{[92], 25}$ Both membranes are functionalized with sulfated or non-sulfated Microciona prolifera thiols to be probed in the presence as well as in the absence of calcium ions. ${ }^{26,27}$ The sulfated epitope is reported to be the native self-recognition unit of Microciona prolifera sponge. The non-sulfated compound is a synthetically modified structure used to evaluate the necessity of the sulfato group for the recognition process in vitro.

We envisioned extracting the number and the strength of interactions as well as to deduce kinetic parameters. The disaccharide structures were synthesized as described by Kamerling and coupled to membranes via maleimide chemistry. ${ }^{[90,94] 28}$

\subsection{Coiled-coil forming peptides $i$-E3Cys and $i$-K3Cys}

To reveal the applicability of the membrane probe setup for the investigation of fusion processes mediated by fusogenic peptides, we investigated the impact of peptide interactions on the magnitude of membrane interaction forces. Similar to the setup introduced by Abdulreda et al., we determined membrane interaction forces after functionalizing membranes with the peptides $i$-E3Cys and $i$-K3Cys depicted in Figure 2.9.

\footnotetext{
${ }^{25}$ Images reprinted from [92] with the permission of the American Chemical Society.

${ }^{26}$ Detailed information about the experimental setups and processing of measurements is given in chapter 4

${ }^{27}$ Synthetic pathways of compounds are given in chapter 8.3.3.

${ }^{28}$ Structures were synthesized by Christian Brand and Ella Kriemen, group of associate professor Dr. D. B. Werz.
} 
In this study, we employed cystein-terminated heptad repeat peptides similar to the helix region of the peptide-lipid structures developed by Robson Marsden et al. in order to form lipopeptide constructs in situ via maleimide chemistry. ${ }^{[94]}$ The synthesis and purification of the peptides as well as the characterization of peptide binding to membranes were carried out by Gesa Pähler.
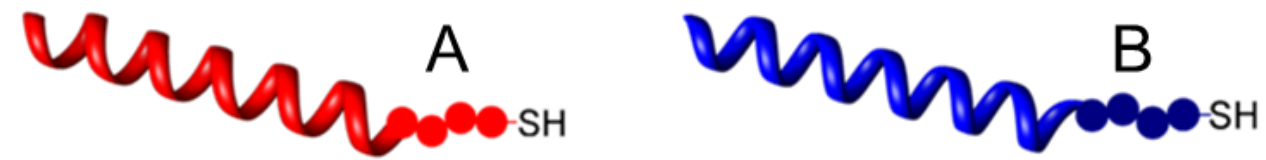

\begin{tabular}{l|r|c|l} 
Peptide & N-terminus & Heptad repeat & \multicolumn{1}{l}{ C-terminus } \\
\hline$i$-K3Cys & Ac-WG- & $(\text { EKLAAIK })_{3}$ & -GGGGC-NH ${ }_{2}$ \\
\hline$i$-E3Cys & Ac- & $(\text { KELAAIE })_{3}$ & -GWGGGC-NH $H_{2}$
\end{tabular}

Figure 2.9: $i$-K3Cys (A) and $i$-E3Cys (B) peptide structures and sequences as synthesized and purified by Gesa Pähler. ${ }^{[95,96], 29}$ A similar setup is reported to be working like a reduced SNARE model. ${ }^{[97],}$ Both peptides are characterized by their heptad repeat domains, which lead to a coiled-coil formation upon combination of $i$-E3Cys and $i$-K3Cys. Due to Cys-tagging the peptides can be bound to maleimide-functionalized surfaces.

Robson Marsden et al. showed that vesicles functionalized with similar lipopeptides interact upon the formation of a heterodimeric coiled-coil between the peptides LPE and LPK (Figure 2.10-A, -B). As depicted in Figure 2.10-C/D, the LPE/LPK model is interpreted as a reduced model mimicking the SNARE-protein mediated fusion promoting full fusion of membranes (Figure 2.10-D). The SNARE motif mediates fusion processes of synaptic vesicles with the synaptic cleft by forming coiled-coils between syntaxin and SNAP-25 residing on the target membrane with synaptobrevin on the vesicle surface. ${ }^{[98]}$ As reported by R. Jahn and others, its recognition domain is an eight-heptad repeat unit, which leads to the interaction of the membranes. ${ }^{[98-100]}$ In contrast, the reduced model of Robson Marsden et al. only requires a dimeric coiled-coil instead of a tetrameric structure.

${ }^{29}$ Drawing provided by G. Pähler, reprinted from [95] with the permission of Elsevier. 
In both cases, an energy gain by hydrophobic interactions of heptad repeat units leads to coiled-coil formation of the involved helices resulting in a mechanical effect on the membranes. The membranes are forced to come over their hydration barrier and outer membrane layers are forced to fuse.
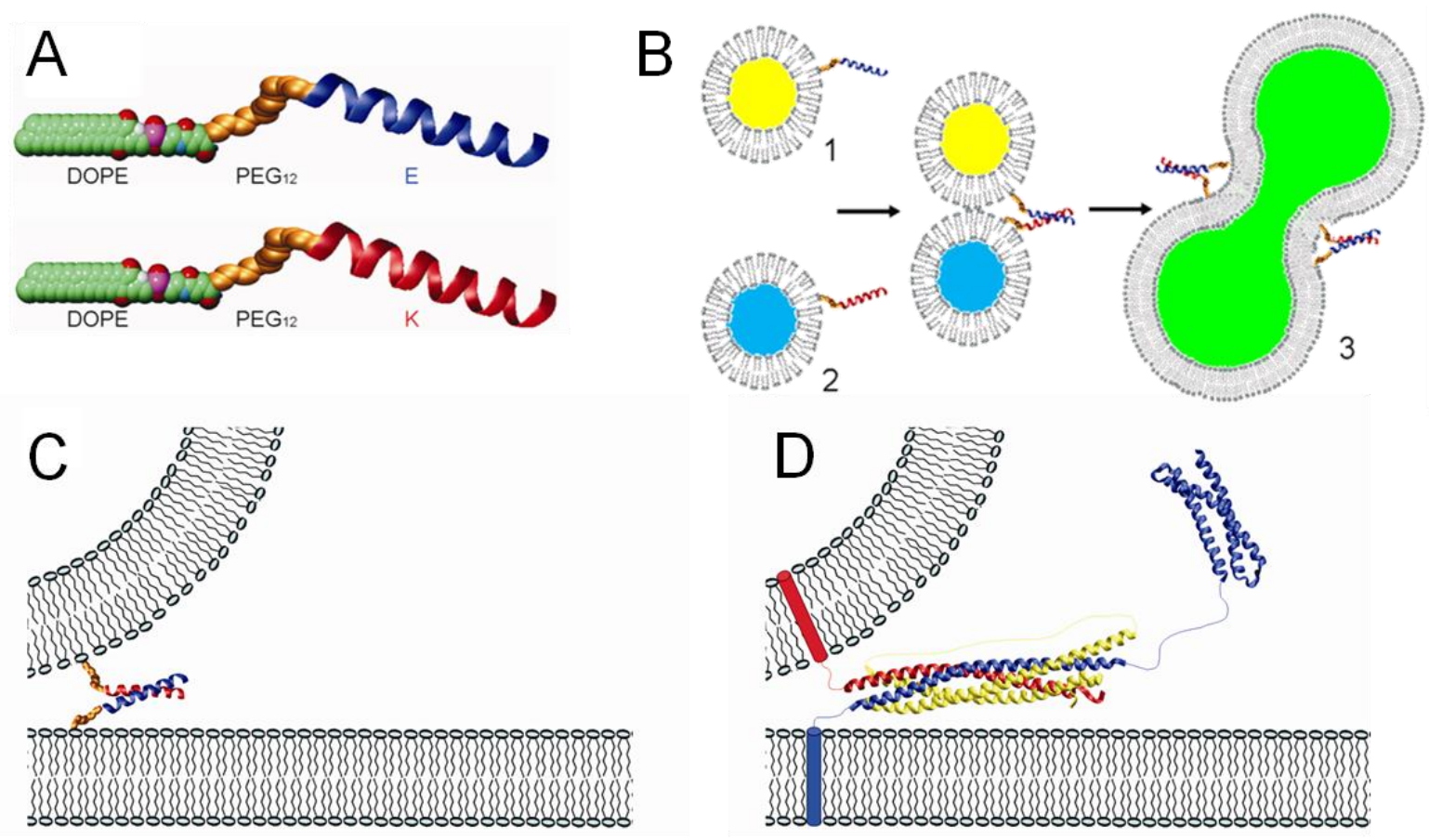

Figure 2.10: The reduced SNARE model introduced by Robson Marsden et al. ${ }^{30,[97]}$ The LPE/LPK peptide interaction (A) mediates vesicle fusion (B). Both peptides consist of a DOPE tail and a polyethylene glycol linker (PEG12), LPE possesses a terminal G(EIAALEK) ${ }_{3}-\mathrm{NH}_{2}$ unit, and LPK a terminal (KIAALKE) ${ }_{3} \mathrm{GW}-\mathrm{NH}_{2}$ unit. Coiled-coil interaction between LPE and LPK anchored in vesicle membranes brings the membranes into close contact resulting in full vesicle fusion characterized by content mixing. The heterodimeric coiled-coil interaction is interpreted as a reduced SNARE model, which essentially mimics the characteristics of vesicle fusion in vivo: LPE/LPK-mediated membrane interaction (C), D: Membrane interaction mediated by the SNARE motif containing transmembrane domain bearing proteins (syntaxin, synaptobrevin) and SNAP25.

With the setup depicted in Figure 2.11 we performed force spectroscopy measurements between membranes doped with i-K3Cys (Figure 2.9-A) and i-E3Cys (Figure 2.9-B) constructs in order to determine if the coiled-coil formation alters membrane interaction forces.

\footnotetext{
${ }^{30}$ High resolution image provided by Alexander Kros, reprinted (slightly modified) from 97. Robson Marsden, $\mathrm{H}$., et al. (2009). "A Reduced SNARE Model for Membrane Fusion." Angewandte Chemie International Edition 48(13): 2330-2333., with the permission of John Wiley and Sons.
} 

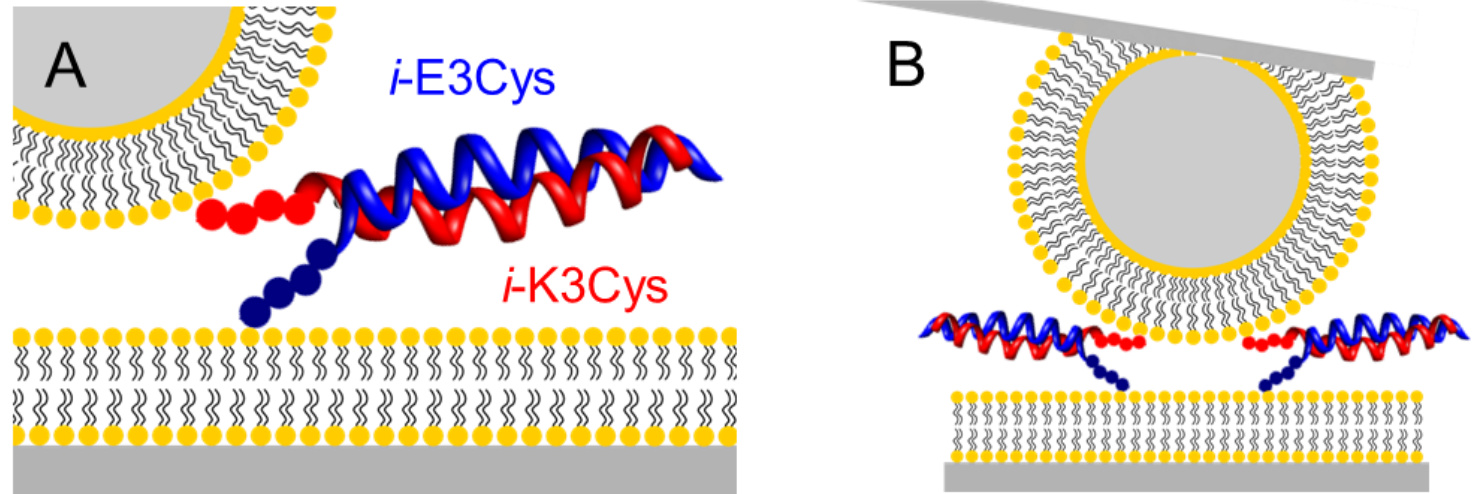

Figure 2.11: Schematic of heterodimeric membrane probe spectroscopy setup to investigate the coiled-coil interaction of peptides $i$-E3Cys (blue) and $i$-K3Cys (red). ${ }^{31}$ The membrane spread on the colloidal probe is functionalized with $i$-K3Cys peptides, whereas $i$-E3Cys peptides form the counterpart at the membrane on the planar support. The heterodimeric coiled-coils (A) act as a mechanical clamp between the membrane on the colloidal probe and the membrane on the flat support $(B) .^{32}$

By using fluid phase membranes, we aimed at determining if peptide diffusion has an impact on the magnitude of interaction forces.

\subsection{E-cadherin structures EC15 and EC12}

As depicted in Figure 1.3 (chapter 1.1), cadherin structures mediate cell-cell contacts by establishing calcium-dependent adherence junctions in order to manage cell adhesion (Figure 2.12-A) as well as cell motion. Classical cadherins such as the E-cadherin (epithelial tissue cadherin) structures involved in this study have a highly conserved ectodomain consisting of five subunits with calcium-binding sites and a cadherin-binding site (Figure 2.12-B). The ectodomain mediates a calcium-dependent recognition process in the extracellular space, whereas the cytoplasmic domain serves as signal transducer in the cell being coupled to catenin molecules, which are in turn connected to the actin cytoskeleton. The cadherin protein possesses a transmembrane domain that anchors the structure in the cellular membrane.

\footnotetext{
${ }^{31}$ Images adapted from [96], with permission of Elsevier.

${ }^{32}$ Detailed information about the experimental setups and processing of measurements is given in chapter 4.
} 

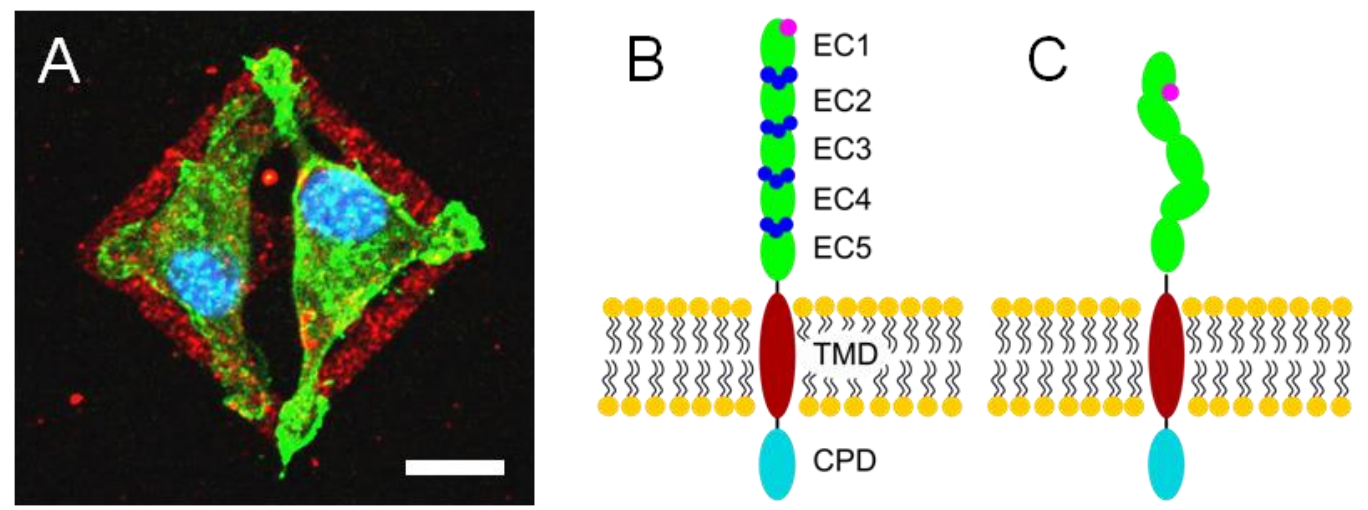

Figure 2.12: Cellular adhesion mediated by cadherin structures. A: Two L cells expressing GFP-labeled Ecadherin constructs adhering to a surface micro-patterned with E cadherin constructs E15 (antibody labeled, red). Nuclei are stained with DAPI (4',6-diamidino-2-phenylindole). The window-like structures are functionalized with EC15 structures (red) to induce cell adhesion. Scale bar: $10 \mu m^{33}$ B, C: Calciumdependence of E-cadherin interaction. ${ }^{34} \mathrm{~A}$ transmembrane (TM) domain anchors the protein in the plasma membrane, whereas the ectodomain containing of five extracellular repeats (EC) mediates cell-cell interaction and the cytoplasmic domain (CPD) regulates signal transduction inside the cell. The functionality of the cadherin ectodomain is highly $\mathrm{Ca}^{2+}$ (green spots) dependent as the availability of binding sites (pink spots) for a homomeric cadherin interaction is enhanced if $\mathrm{Ca}^{2+}$ ions are bound.

Cadherin-mediated cell-cell contacts are characterized by a homomeric binding of ectodomain structures on two opposing cells. The interaction is believed to be established by two kinds of interactions, the cis interaction between two monomers on the surface of cell 1 and the trans interaction between structures on cell 1 and cell 2 (Figure 2.13).

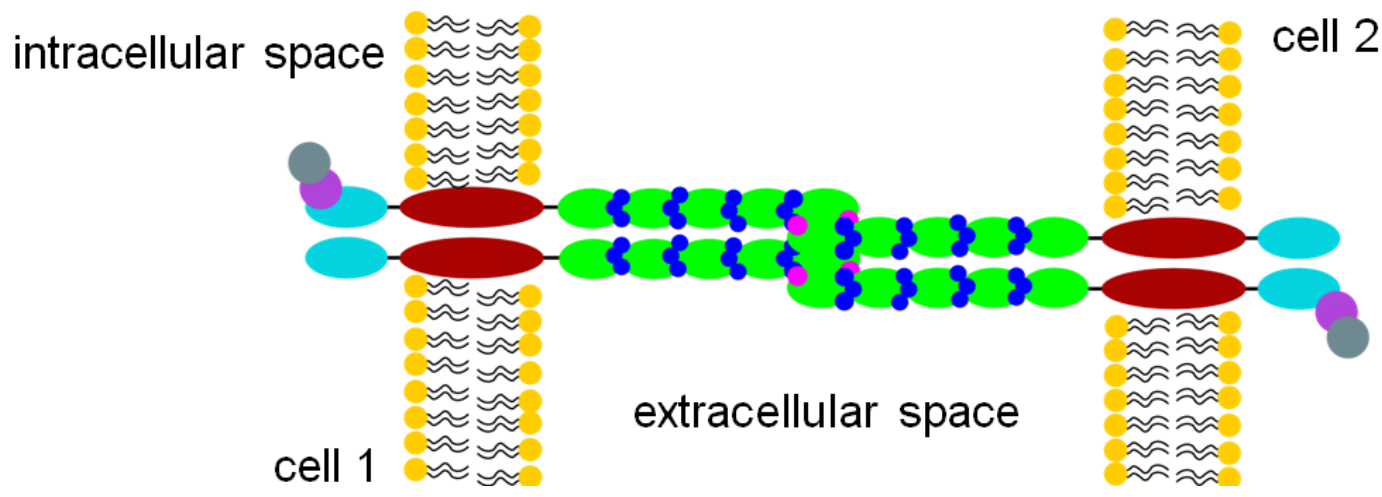

Figure 2.13:. Trans interaction of cis coupled ectodomains on two adjacent cells. The intracellular part of the adherence junctions is coupled to actin fibers via catenins. Trans coupling denotes the intercellular part of the interaction, whereas cis coupling describes the interaction between adjacent cadherin monomers on the same cell.

\footnotetext{
${ }^{33}$ Image provided by Dagmar Fichtner, Karlsruhe, and used with her permission.

${ }^{34}$ Adapted from 101. Bayas, M. V., et al. (2006). "Lifetime Measurements Reveal Kinetic Differences between Homophilic Cadherin Bonds." Biophysical Journal 90(4): 1385-1395.
} 
Cis coupling is realized by coupling of the outer extracellular repeat (EC1) of one cadherin monomer to the EC2 domain of a monomer on the same cell-surface (Figure 2.14-A/B).
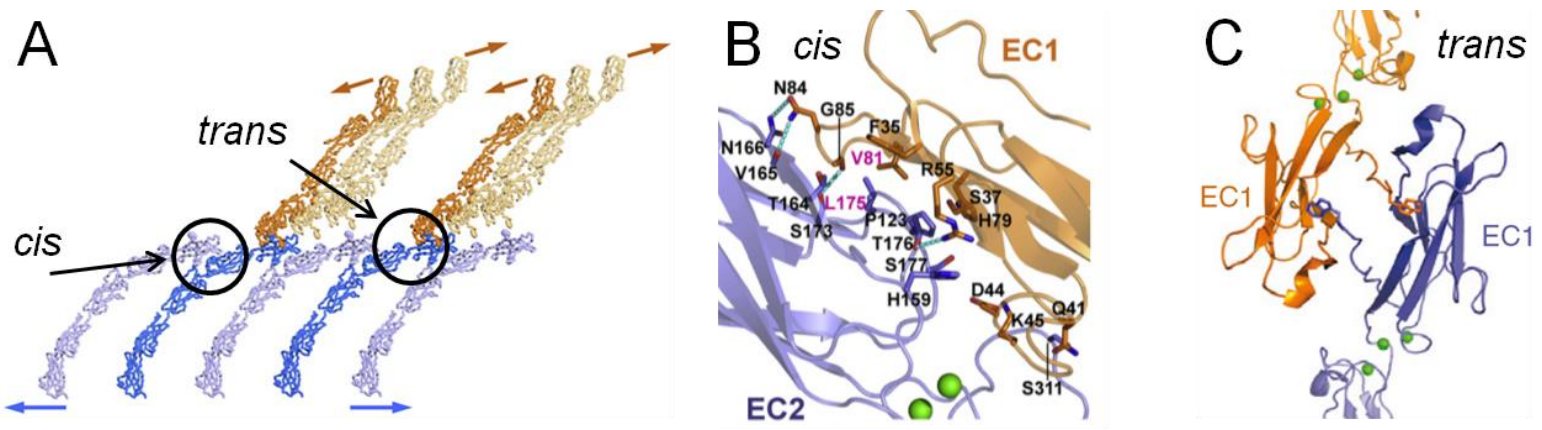

Figure 2.14: E-cadherin cis and trans interactions. ${ }^{[102,103]}, 35$ A two dimensional lattice is formed upon cis and trans interactions between cadherin ectodomains between opposing cells $(A) .{ }^{[102]}$ Protein structures involved in the cis interaction between an EC1 and an EC2 unit on the same cell surface are depicted in $B^{[103]}$ C: The insertion of Trp2 residues of the EC1 domain of one cadherin structure into a hydrophobic grove of an EC1 domain of a cadherin structure on the surface of a second cell leads to the so called trans interactions. ${ }^{[102]}$

Trans coupling is realized by the insertion of a Trp2 residue at EC1 units into hydrophobic groves of EC1 of cadherin structure of an opposed cell (Figure 2.14-A/C).

However, the relevance of cis coupling and with that the role of dimerization for intercellular adhesion still is a matter of debate. Our aim is to elucidate whether cadherin monomer interactions are sufficient for successful formation of cell-cell contacts.
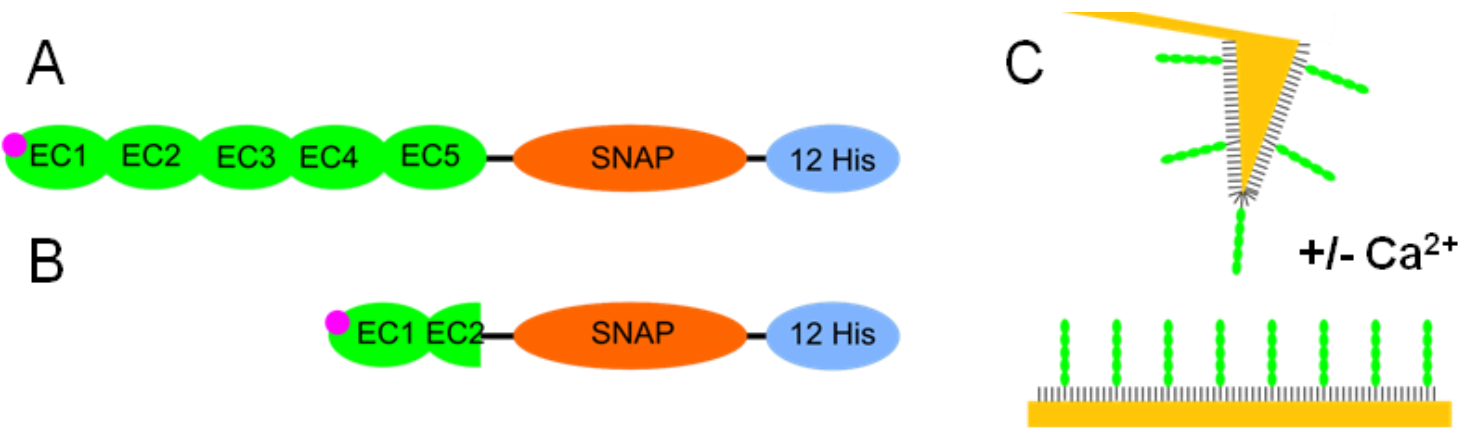

Figure 2.15: Probing EC15 and EC12 monomers in order to determine the impact of ectodomains and dimerization on adhesion strength. A, B: EC15 and EC12 constructs as expressed and purified by Engin et $a l .{ }^{[104], 36}$ Structures carry a SNAP domain, which is used to couple them covalently to benzylguanine (BG)functionalized substrates in a site-specific manner. EC15 corresponds to the full-length ectodomain species, whereas EC12 only possesses EC1 and a truncated EC2. This component can be used to determine the impact of full EC12 as well as EC3 to EC5 for full cadherin functionality. C: Schematic of the single molecule force spectroscopy setup used to probe the homomeric interaction between cadherin domains (SNAP unit and His-tag not depicted here).

\footnotetext{
${ }^{35}$ Images reprinted from [102] and [103] with permissions of Elsevier.

${ }^{36}$ Adapted from original images provided by Dagmar Fichtner and Sinem Engin, Karlsruhe.
} 
In order to probe the impact of monomer surface density on adhesion strength between two opposing substrates we envision a model system depicted in Figure 2.15-C. In collaboration with the group of Prof. D. Wedlich, we employed the full-length ectodomain cadherin constructs $E C 15$ as well as truncated EC12 constructs (Figure 2.15 ) in order to screen the necessity of ectodomains EC3 to EC5 for successful cis and trans interaction.

An important feature of the system is the covalent immobilization of compounds, which allows a reliable dosage of cadherin monomer constructs on the cantilever and substrate surface. To realize this, we used constructs disposing of SNAP tags allowing for a stable bond formation to benzylguanine-functionalized surfaces.

In addition, we were aiming at the relevance of calcium ions for the availability of $\operatorname{Trp}^{2}$ binding sites for interaction with the hydrophobic grove of opposing cadherin ectodomains. Hence, we performed force spectroscopy measurements in the presence and absence of calcium ions. 


\section{Materials and Methods}

The first part of this chapter briefly describes the techniques that were used in this study and provides details about the materials employed. The second chapter summarizes experimental details of membrane probe spectroscopy measurements.

\subsection{Atomic force microscopy}

Atomic force microscopy (AFM) imaging was used to control the integrity of membranes prior to membrane probe spectroscopy measurements (see also chapter 5.1.4) as well as for the characterization of substrates used for cadherin experiments (compare chapters 6.1).

\section{Experimental setup}

The general setup of an atomic force microscope (Figure 3.1) is as follows: a sample in a sample holder is mounted to the microscope stage and a sharp tip on a cantilever is brought into close proximity to the surface. ${ }^{[05]}$

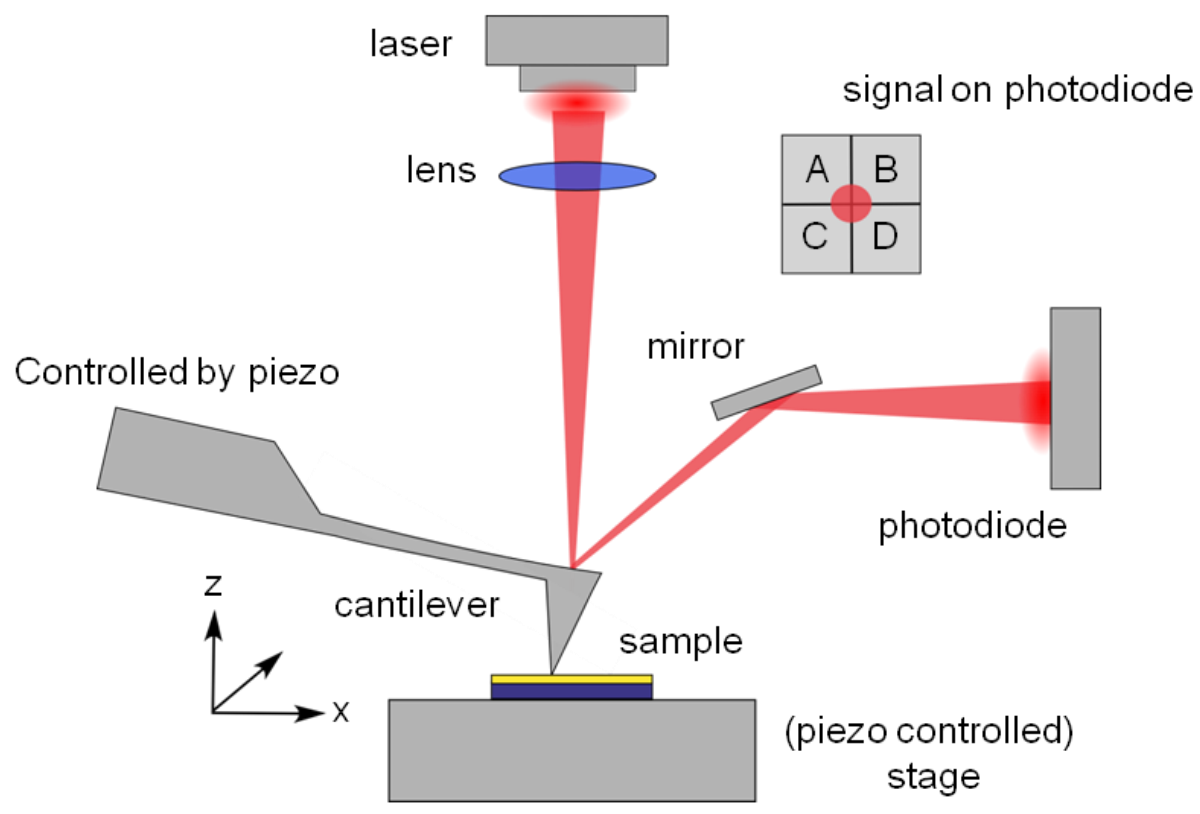

Figure 3.1: The atomic force microscope setup. ${ }^{[105]}$ Piezo elements are used to raster-scan the cantilever over a sample surface or to move a sample under the cantilever as depicted here. The deflection of the cantilever is directly coupled to the voltage detected on a split photodiode and can be used as feedback signal. After calibration of the system this signal can be converted into force values. 
To detect the interaction of cantilever and sample, the optical lever system is used. Here, a focused laser beam is aligned on the reflective backside of the cantilever and the reflected light is collected on a voltage-sensitive photodiode (Figure 3.1). The cantilever is moved in a horizontal direction relative to the sample surface or vice versa. Usually, a feedback system is used to detect motions of the cantilever upon interaction of the sharp tip with the sample surface, which are then used to calculate a topographic image of the surface.

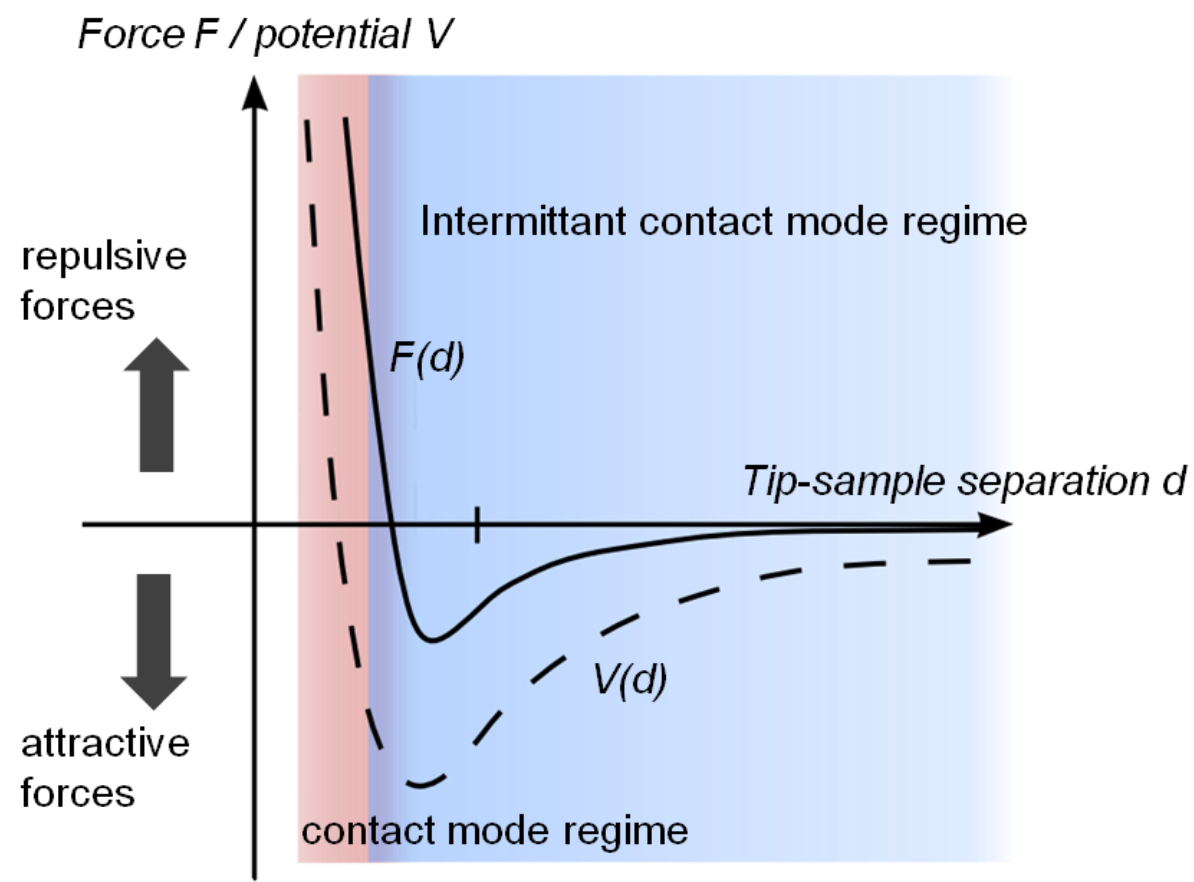

Figure 3.2: Interaction potential and resulting force as a function of the tip-sample separation. ${ }^{37}$ Relevant tip-sample distances and measured forces for contact and intermittent contact mode are indicated. Contact mode is working in the repulsive force regime, whereas intermittent contact mode is characterized by an alternating motion between repulsive and attractive regimes.

While moving cantilever and sample relative to each other the cantilever bends in response to attractive or repulsive forces acting. Interaction potentials and resulting cantilever motion are depicted in Figure 3.2. In close proximity to the surface repulsive steric forces act on the cantilever whereas in a short range $>1 \mathrm{~nm}$ attractive forces such as Van der Waals forces dominate the cantilever deflection.

\footnotetext{
${ }^{37}$ Image adapted from 106. Künneke, S. (2003). Kontaktmechanik und Strukturierung von festkörperunterstützen Lipidmembranen. Institut für Physikalische Chemie, Fachbereich Chemie und Pharmazie. Mainz, Johannes Gutenberg-Universität in Mainz., http://ubm.opus.hbz-nrw.de/volltexte/2003/424/
} 
The difference of light intensities on different segments of a photodiode, a position-sensitive detector (PSD) Figure 3.1), during a cantilever approach-retraction cycle is used to calculate the effective motions of the cantilever.

\section{Calibration of the system}

To convert voltage values $V$ into deflection values $Z_{c}$ the inverted optical lever sensitivity in$V O L S$ given in $\mathrm{V} / \mathrm{nm}$ is measured during the measurement by using the thermal noise method (chapter 3.1.2). The cantilever is treated as a harmonic oscillator and its first vibration mode is used for the calibration of the optical lever system. The optical lever sensitivity is calculated from the slope of the deflection of a cantilever on a hard substrate and the corresponding signal detected on the photodiode by fitting the slope of the raw data (Figure 3.3-A).
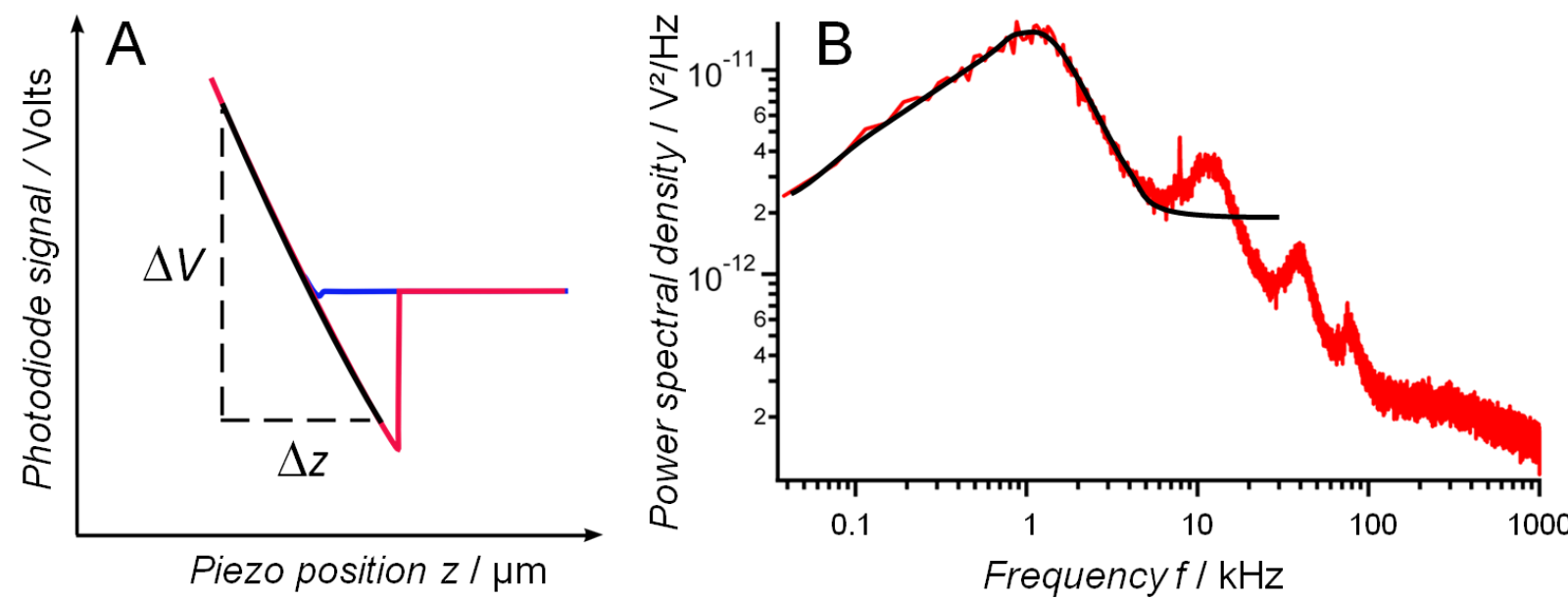

Figure 3.3: Calibration of a cantilever in an optical lever setup. A: Cantilever deflection plotted versus piezo movement during a cantilever contact to a hard surface. The slope of the curve is used to determine the sensitivity of the optical lever setup. B: Thermal noise spectrum of a MLCT cantilever in liquid. The first resonance is the cantilever is used to extract the mean square deflection of the cantilever $\left\langle\delta V^{2}\right\rangle$.

By using the square of the cantilever deflection of the first vibration mode of the cantilever, the spring constant of the cantilever $k_{c}$ can be calculated using the equipartition theorem (Equation 1). ${ }^{[107]}$ In this equation, the parameter $\left\langle\Delta Z_{c}^{2}\right\rangle$ represents the mean square deflection of the cantilever due to thermal fluctuations and $k_{b}$ is the Boltzmann constant. 


$$
\frac{1}{2} k_{c}\left\langle\Delta Z_{c}^{2}\right\rangle=\frac{1}{2} k_{b} T \Rightarrow k_{c}=\frac{k_{b} T}{\left\langle\Delta Z_{c}^{2}\right\rangle}
$$

\section{Equation $1^{38}$}

In our setup, Equation 2 is used to determine kc using the voltage expression of the mean square deflection of the cantilever $\delta V^{2}$, the sensitivity of the optical lever system InvOLS, and a correction factor $\chi$.

$$
k_{c}=\frac{k_{B} T}{\left\langle\delta V^{2} \chi^{2} \ln v O L S^{2}\right\rangle} \quad \text { with invOLS }=\frac{\Delta z}{\Delta U}
$$

Equation 2

Due to the linear relationship of stiffness (spring constant $k_{c}$ usually given in $\mathrm{pN} / \mathrm{nm}$ ) and deflection $d$ (in $\mathrm{nm}$ ) of the cantilever given by Hooke's law (Equation 3), the forces $F$ (in $N$ ) acting on the lever can be extracted from the measurements.

$$
F=k_{c} d
$$

Equation 3

The tip-sample distance $d$ is calculated by adding the deflection of the cantilever $d_{\text {cantilever }}$ to the distance travelled by the piezo $z$ in order to change the cantilevers position.

$$
d=z+d_{\text {Cantilever }}
$$

\section{Equation 4}

During imaging the sample is raster-scanned with the cantilever tip to give a 3-dimensional image of the surface. Measurements can be performed in vacuum, air or in liquid environment. The force resolution limit is a few picoNewton even in aqueous environment and largely depends on external vibrations and depends on the square root of the spring constant $k_{c}$ : $\Delta F \propto \sqrt{k_{c}}$ Considering length scales determined on a sample, the lateral resolution is $1 \mathrm{~nm}$ and the vertical resolution ranges of the Angstrøm regime.

\footnotetext{
${ }^{38}$ A list of all parameters can be found in chapter 8.2 , page 129. 


\subsubsection{Imaging modes}

\section{Principle}

For imaging biological surfaces one uses intermittent contact (Figure 3.4-A) mode or contact mode (Figure 3.4-B). In intermittent contact mode (also called AC mode or tapping mode) the cantilever is driven to oscillate at a constant amplitude value. As the tip is only in contact with the surface for a time in the range of milliseconds is called intermittent contact mode.
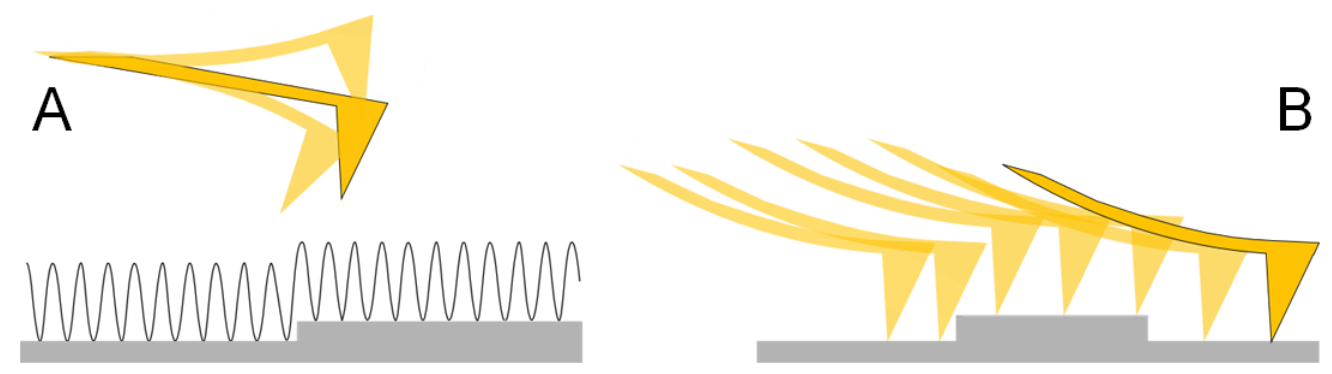

Figure 3.4: Basic AFM imaging techniques. In AC mode the cantilever is driven at a constant frequency and the contact time to the surface is relatively low (A). In contact mode the deflection of the cantilever is recorded as the feedback signal. In contrast to AC mode this mode is used for relatively stiff samples (B).

It is mainly used for imaging of sensitive or soft samples due to reduced lateral interactions compared to contact mode. In contact mode, the cantilever is brought into repulsive contact with the sample and raster-scanned over the surface at a constant deflection value. In order to reduce the force applied to the surface, typical force constants of cantilevers for this mode are relatively low $(0.006$ to $0.1 \mathrm{~N} / \mathrm{m})$. In contrast, typical force constants of tapping mode cantilevers to operate in air or vacuum are $1-100 \mathrm{~N} / \mathrm{m}$. Their resonance frequency is of an order of magnitude higher $(\sim 300 \mathrm{kHz})$ than for contact mode cantilevers as higher frequency values are needed for better $\mathrm{Q}$ value and hence an improved signal-to-noise ratio.

\section{Materials}

For all measurements we used a commercial AFM setup (MFP3D ${ }^{\mathrm{TM}}$ Stand Alone, Asylum Research, Santa Barbara, CA, USA). Used probes were Bio-Levers (BL-RC150VB, Olympus, Hamburg, Germany) as well as MLCT, MLCT-O10, and MSCT cantilevers (Bruker AFM Probes, Camarillo, CA, USA). ${ }^{39}$

\footnotetext{
${ }^{39} \mathrm{~A}$ list of all cantilevers used in this study can be found in chapter 8.4 on page 136. 
Colloidal probe cantilevers were manufactured by using MLCT-O10 cantilevers and borosilicate beads as described in chapter 3.4.1. A collection of cantilever properties is given in chapter 8.4. Different fluid chambers were used as sample holders.

\subsubsection{Force spectroscopy}

Force spectroscopic measurements were used to probe interactions between membranes, which were functionalized with $\mathrm{Ni}^{2+}-\mathrm{NTA}$ and $\mathrm{H} 6$ (chapters 2.1, 3.5, and 5.3), Microciona prolifera disaccharides (chapters 2.2, 3.6, and 5.4) as well as peptides $i$-E3Cys and $i$-K3Cys (chapters 2.3, 3.7, and 0). Furthermore, the homomeric interaction of E-cadherin ectodomains was investigated in a single molecule force spectroscopy setup (chapters 2.4, 3.8, and 6).

\section{Principle}

In force spectroscopy measurements, a probe is brought in contact with a surface and retracted. The magnitude of forces acting between tip and sample during the force-distance cycle depend on the stiffness of the cantilever, the probe radius, the probe material, and the chemical environment during the measurement. If interactions between tip and sample are probed, bonds between probe and sample are loaded by a force ramp defined by the applied pulling speed $v$ and the stiffness of the cantilever $k_{c 1}$ :

$$
\begin{gathered}
F=k_{c} \cdot v \cdot t .^{[107]} \\
\text { Equation } 5
\end{gathered}
$$

The usually applied forces range from a few PicoNewton to several NanoNewton. Figure 3.5 demonstrates a force spectroscopy measurement. A cantilever (1) is brought in contact with a surface (2) until it reaches a certain deflection value (3). Afterwards, the cantilever is retracted from the surface and may exhibit adhesion leading to a negative bending (4) before it snaps back to the line of zero deflection (5). Force spectroscopy can be used to indent samples and to determine elastic properties of the sample. In this kind of measurement, the approach of tip to sample is used (blue curve in Figure 3.5) is analyzed and parameters such as Young modulus $E$, apparent spring constant $\kappa_{a p p}$, and lateral tension $\sigma$ can be obtained. ${ }^{[16,25,108]}$ 
By analyzing the retraction of the probe from the sample (red curve in Figure 3.5) one can extract the kinetics of isolated receptor-ligand pairs (single-molecule force spectroscopy) or of bond clusters. ${ }^{[65]}$

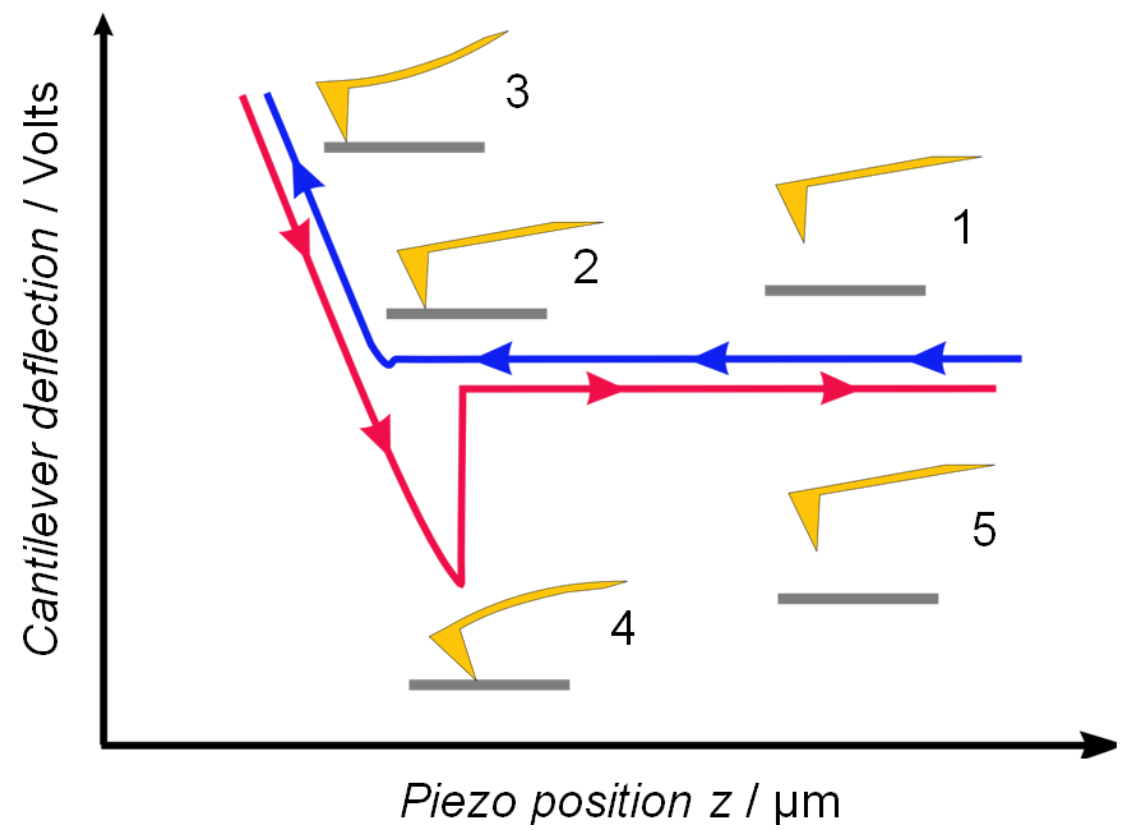

Figure 3.5: Raw data as recorded with a force spectroscopy setup. ${ }^{40}$ A: Deflection-versus-piezo distance curve on a hard substrate. The cantilever is approached to the surface (blue curve, 1), gets into contact with the surface (2) and bends upwards in repulsive contact with the sample (3). Upon retraction of the cantilever, it bends downwards as adhesive forces act on the lever (4) before it snaps back to zero deflection (5).

Forces can be applied over a certain distance, which means that energy is acting on the bond altering its potential. By doing this, one is able to gather information about the strength and the binding kinetics of the bond. Furthermore, cooperativity and reversibility of bond formation can be quantified. ${ }^{[109]}$ One major aspect of force spectroscopy measurements is the determination and quantification of inter- and intramolecular forces.

The method has become one of the most important tools to study aspects of biological interaction processes as these can be studied in physiological conditions without the need to label the structures concerned in the process. ${ }^{[65]}$

\footnotetext{
${ }^{40}$ Adapted from 65. Bizzarri, A. R. and Cannistraro, S. (2010). "The application of atomic force spectroscopy to the study of biological complexes undergoing a biorecognition process." Chemical Society Reviews 39(2): 734-749.
} 


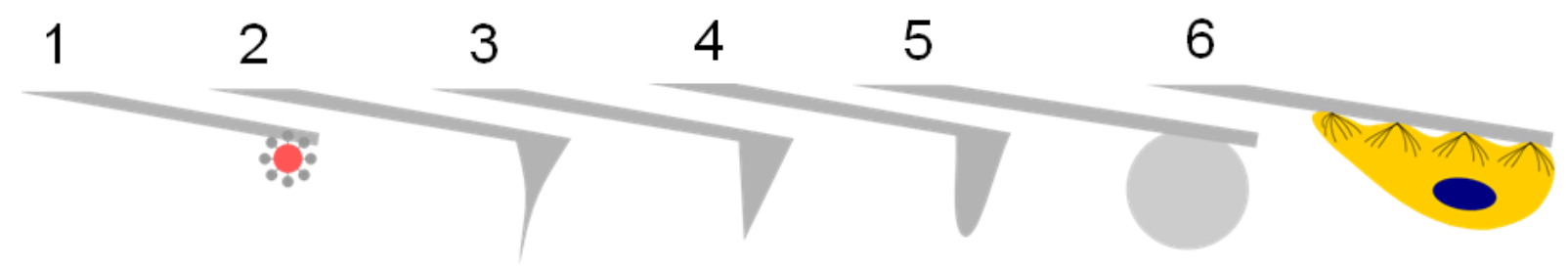

Figure 3.6: Variability of AFM probes. Tip geometries as well as probe material can be varied. Conventional cantilevers are available with different tip radii (2-5) ranging from $10 \mathrm{~nm}$ to several $\mu \mathrm{m}$ probe radius. Furthermore cantilevers can be equipped with virus (1), bacterium (not shown), or cell probes (6).

A further great advantage of the AFM for force spectroscopy measurements is the variability of the experimental setup. They can be designed using different tip radii, tip/cantilever materials and lengths, and probe materials such as diamond, carbon nanotubes, spheres, or even viruses, bacteria and cells (Figure 3.6).

\section{Materials}

In order to optimize the force resolution, cantilevers with low force constants ranging from 0.005 to $0.1 \mathrm{~N} / \mathrm{m}$ are frequently employed. For membrane probe spectroscopy measurements MLCT-C and MLCT-E cantilevers with $k_{c}$ values of $0.01 \mathrm{~N} / \mathrm{m}$, and $0.1 \mathrm{~N} / \mathrm{m}$, respectively, were used. Colloidal probe cantilevers were fabricated from MLCT-O10 levers as described in chapter 3.4.1. In the single molecule setup used for the investigation of cadherin-cadherin interactions (chapter 0) we used Biolevers with nominal $k_{c}$ values of $0.06 \mathrm{~N} / \mathrm{m}$. Technical data about cantilevers is summarized in chapter 8.4.

\subsection{Fluorescence microscopy}

Epifluorescence microscopy and confocal laser scanning microscopy (CLSM) were used to control the membrane coverage of the colloidal probe (chapters 3.4 .3 and 5.1.2). Lateral mobility of the membrane was determined by applying the fluorescence recovery after photobleaching (FRAP) technique. 


\section{Principle}

In biology, fluorescence microscopy setups are usually used to exactly localize a compound, which has been specifically labeling beforehand. This particular compound is labeled with a fluorescence active marker possessing a delocalized $\pi$ electron system. Electrons can by excited to the $S_{2}$ level and emit fluorescent light be relaxing from $S_{1}$ to the ground state $S_{0}$. In lipid membranes 0.1 to $1 \mathrm{~mol} \%$ of fluorescence-labeled lipid is enough to label the whole structure (compare chapter 5.1.2). For staining of proteins secondary antibody staining can be used (see chapter 3.8.2).

\section{Epifluorescence microscopy and confocal laser scanning microscopy}

An epifluorescence setup was used to get qualitative information about fluorescently labeled surfaces. A confocal fluorescence microscope setup makes use of pinholes, which allows for the selective excitation of fluorophors in the specimen. The setup allows for a pinpoint illumination of the sample, which means that a resolution of $200 \mathrm{~nm}$ can be reached without meeting the disadvantages of an epifluorescence setup. Every layer of a sample can be scanned point-wise, which means that 2D and 3D images can be obtained. Due to the laser power provided with this setup it can be used to locally bleach the sample and determine the amount of molecules diffusing back into the region of interest.

\section{Fluorescence recovery after photobleaching}

To determine the lateral mobility of the membrane spread on a colloidal probe we used fluorescence recovery after photobleaching (FRAP). A micrometer-sized spot (region of interest, ROI) was bleached on the very top of the probe, which was functionalized with a $\beta$-Bodipy ${ }^{\circledR}$ $500 / 510 C_{12}-H P C$ (BY)-labeled fluid phase lipid membrane (Figure 3.7). The fluorescence intensity during bleaching and fluorescence recovery is detected at the bleaching spot and at a reference spot and can then be used to calculate mobile and immobile fractions of the sample as well as the time needed for fluorescence recovery in the ROI. 


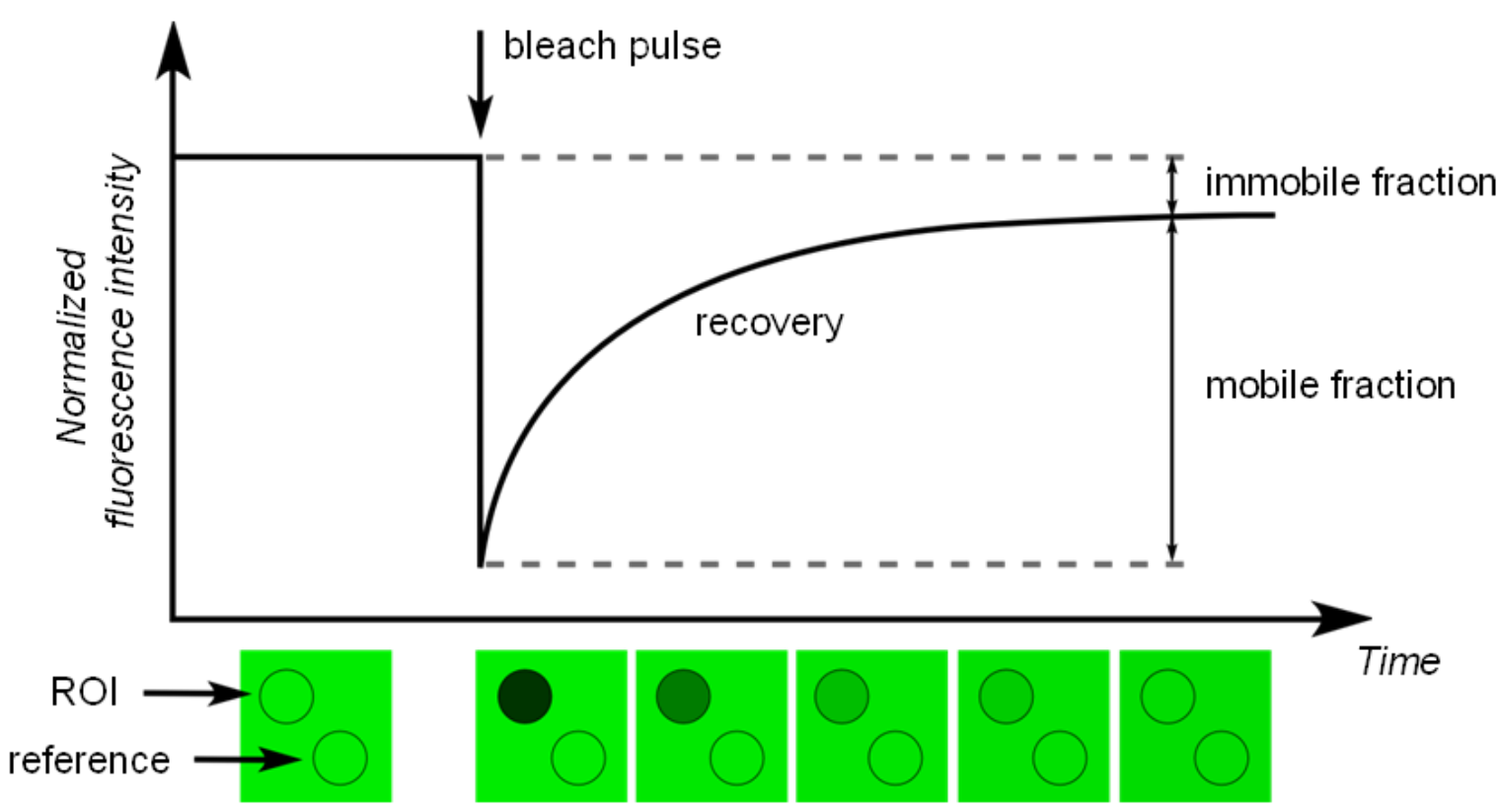

Figure 3.7: Principle of fluorescence recovery after photobleaching (FRAP) experiments. ${ }^{41}$ A strong laser pulse is used to bleach the fluorophors in a circular region of interest (ROI). From the fluorescence intensity signal of the ROI and the reference spot (non-bleached area) the diffusion coefficient of fluorescent molecules can be determined.

The diffusion coefficient $D$ of the bleached species in its matrix is determined by using a suitable model such as the Axelrod function. ${ }^{[111]}$

\section{Materials}

Fluorescent lipids as used in this study are depicted in Figure 3.8. Fluorophor entities are positioned either at the head group of a lipid (Texas Red ${ }^{\circledR}$ DHPE/Sulforhodamine 101 DHPE, "TR", 1,2-dihexadecanoyl-sn-glycero-3-phosphoethanol-amine, triethylammonium salt, Figure 3.8-A) or incorporated into the fatty acid tail (Figure 3.8-B, $\beta$-Bodipy ${ }^{\circledR} 500 / 510 C_{12}$-HPC (2-(4.4-difluoro-5-methyl-4-bora-3a,4a-diaza-s-indacene-3-dodecanoyl)-1-hexa-decano-ylsn-glycero-3-phosphocholine, Invitrogen, Karlsruhe, Germany)). The fluorescent lipids are soluble in chloroform and are used to fluorescently label lipid membranes with a concentration of 0.1 to $1 \mathrm{~mol} \%$.

\footnotetext{
${ }^{41}$ Adapted from 110. Kocun, M. (2011). Mechanical properties of pore-spanning membranes prepared from giant vesicles. Institute for Physical Chemistry. Göttingen, Georg August University, permission for usage by M. Kocun.
} 

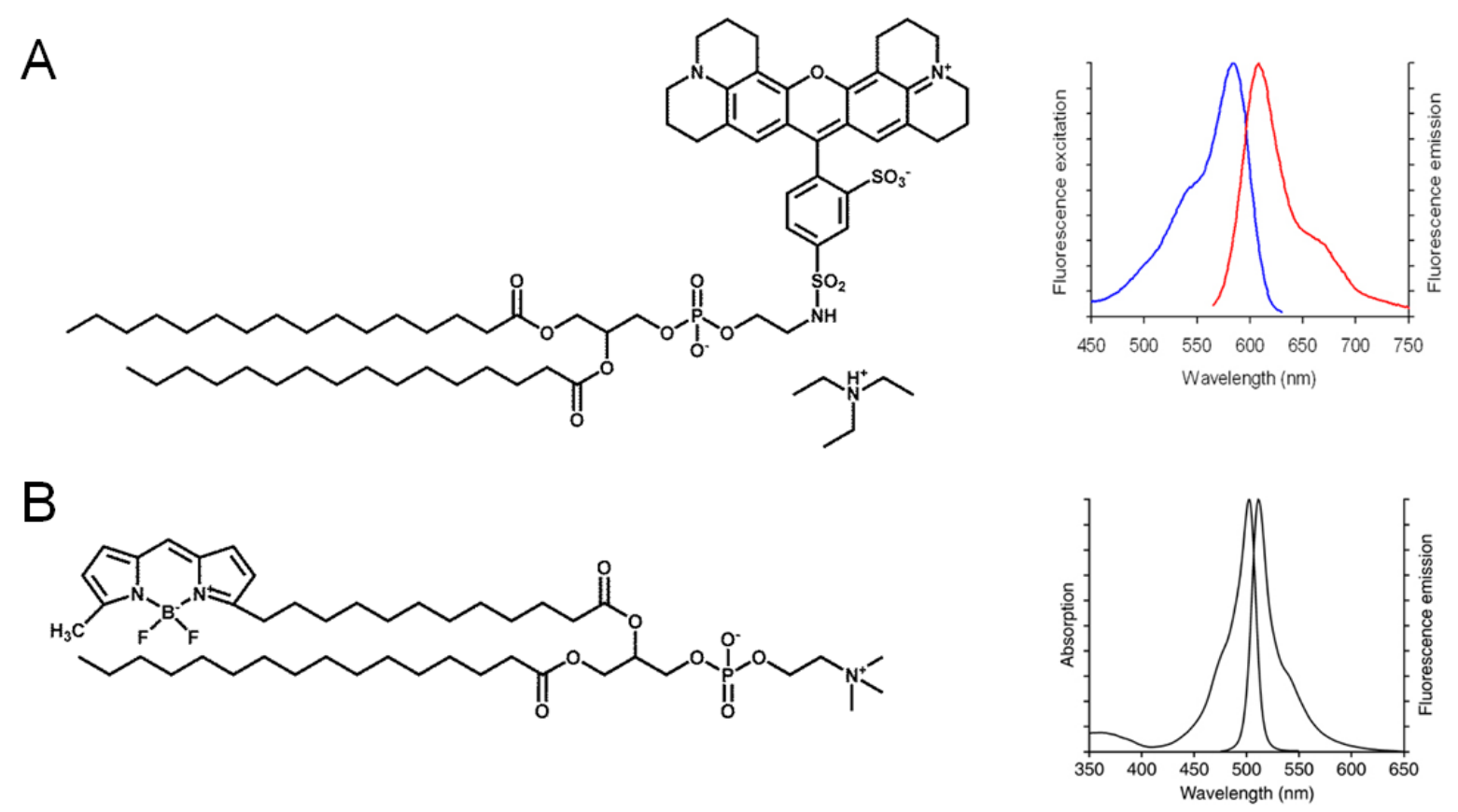

Figure 3.8: Fluorescent lipids used in this study. A: Sulforhodamine $101 \mathrm{DHPE} / \mathrm{Texas}$ Red ${ }^{\circledR} \mathrm{DHPE}$, chemical structure and excitation (blue) /emission (red) spectra, ${ }^{42}$ fluorescent label in the hydrophilic part of the lipid. B: $\beta$-Bodipy ${ }^{\circledR} C_{12}$ HPC, chemical structure and excitation/emission spectra, ${ }^{42}$ fluorescent label in the hydrophobic part of the structure.

As Bodipy fluorophors readily undergo bleaching at higher laser intensities they are suitable for being used for FRAP measurements (previous paragraph). For analyzing the FRAP data, we used a model developed by Axelrod. ${ }^{[111]}$ We used a BX51 epifluorescence microscope setup (Olympus, Hamburg, Germany) equipped with different lenses (10x, NA =0.3; 40x, $N A=0.8 ; 100 x, N A=1.0$ ) and an LSM 710 (Zeiss, Jena, Germany) equipped with a $63 x$ magnification objective.

\footnotetext{
${ }^{42}$ Spectra provided by www.invitrogen.com
} 


\subsection{Reflectometric interference spectroscopy}

\section{Principle}

Reflectometry interference spectroscopy is a label-free method to detect the adsorption of thin layers (Figure 3.9-A to $-B$ ) by analyzing a change in reflectometry, which occurs due to the different refractive indices of adsorbed layers. ${ }^{[112-115]}$
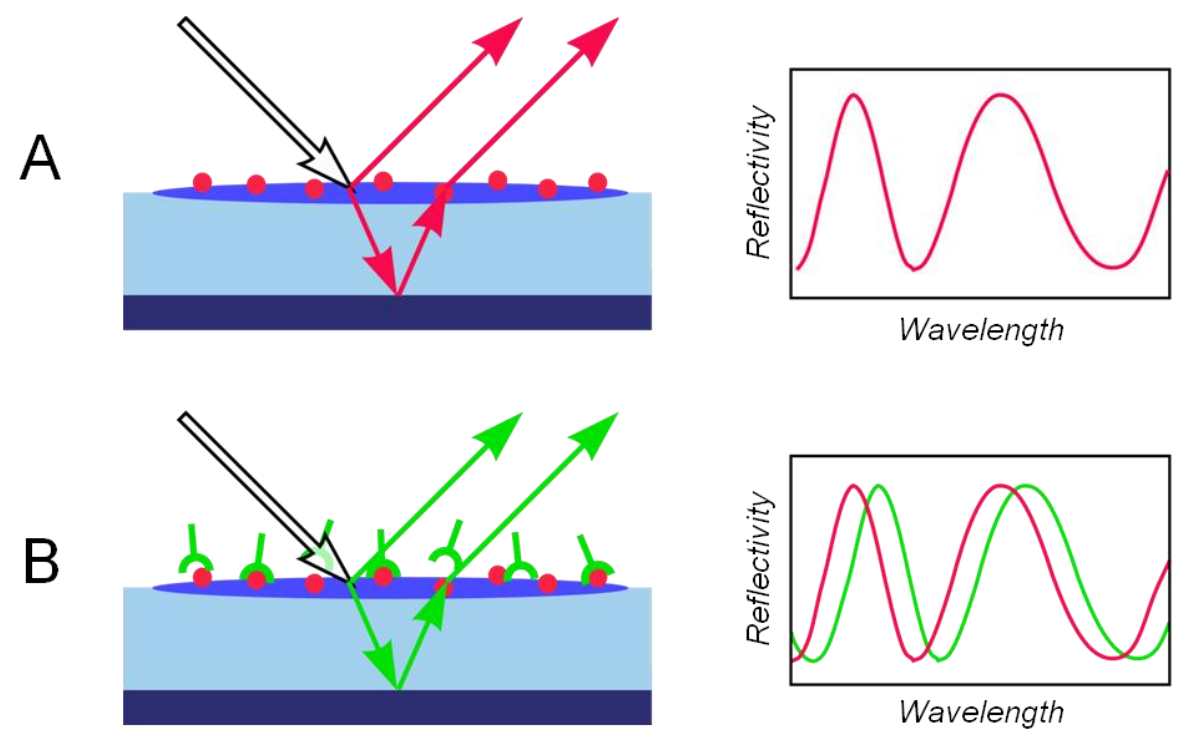

Figure 3.9: Reflectometry interference spectroscopy setup as used to detect binding events by analyzing the optical properties of the surface. ${ }^{43} \mathrm{~A}$ change in reflectivity is detected upon binding of molecules (A to B) to the surface and can be analyzed to give optical thickness (OT) changes.

It is used for measurements in the gas phase, the detection of herbicides in water, for antigen-antibody binding assays, for separation of chiral substances, and even for detection of cell adhesion. ${ }^{[116-120]}$

In our study, a silicon wafer with an oxide layer of $5 \mu \mathrm{m}$ thickness is used as a substrate for vesicle spreading and covalent binding of molecules. The thickness of adsorbed layers with a refractive index of $n_{i}$ can be determined by detecting the reflectivity of the surface and by calculating optical thickness OT values while accounting for the Fresnel coefficients given by and the following relation:

\footnotetext{
${ }^{43}$ Schematic based on an idea by Milena Stephan and on Figure 1 in 115. Gauglitz, G. (2010). "Direct optical detection in bioanalysis: an update." Analytical and Bioanalytical Chemistry 398(6): 2363-2372.
} 


\section{Materials}

Silicon wafers with an oxide layer of $5 \mu \mathrm{m}$ thickness were used to guarantee sufficient interference. Spectra were recorded with a NanoCalc-2000-UV/Vis/NIR spectrometer (Ocean Optics, Ostfildern, Germany) and were analyzed by a Matlab tool designed to determine OT values.

\subsection{Membrane probe microscopy: preparation of probe system}

\subsubsection{Manufacturing of colloidal probe cantilevers}

Colloidal probe cantilevers were prepared by attaching a borosilicate glass microsphere with a diameter of $15 \mu \mathrm{m}$ (Duke borosilicate glass 9015, Duke Scientific Corporation, Palo Alto, CA, USA) to a tipless MLCT-O10 cantilever (Bruker AFM Probes, Camarillo, CA, USA) using epoxy resin at a temperature of above $90{ }^{\circ} \mathrm{C}$ (Epikote 1004, Brenntag $\mathrm{GmbH}$, Mühlheim, Germany ${ }^{44}$, Figure 3.10-A). In detail, we used an upright light microscope (Olympus BX 51, Hamburg, Germany) with a 20x magnification equipped with a nanomanipulator (MM3A-LS, Kleindiek Nanotechnik GmbH, Reutlingen, Germany, Figure 3.11).
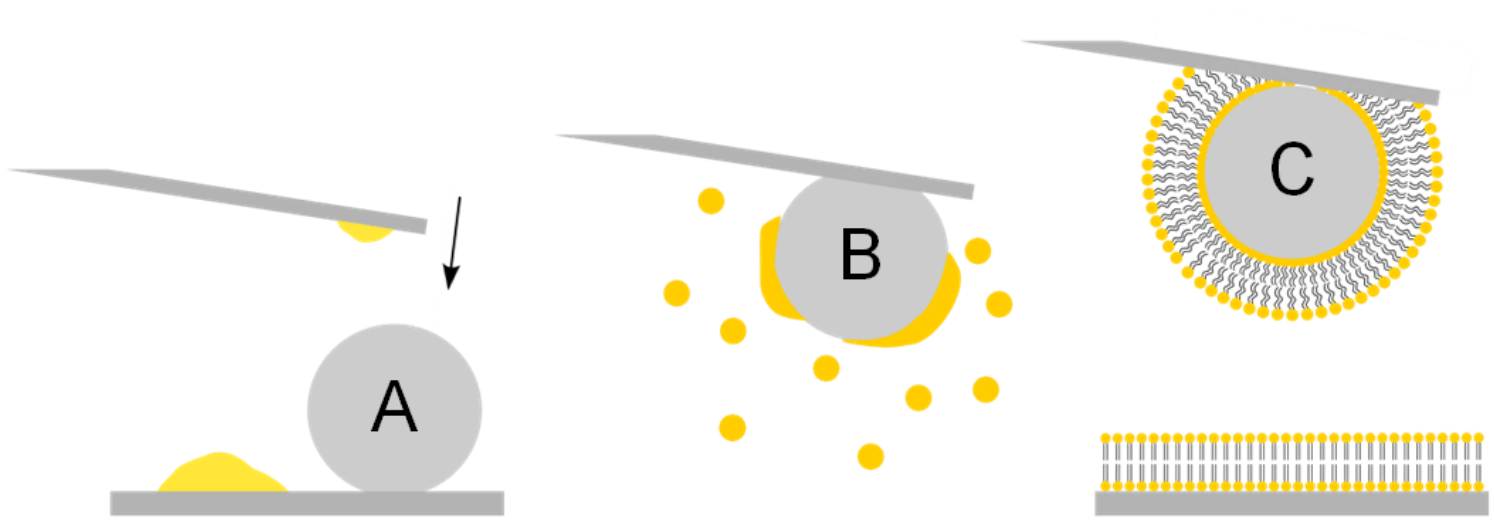

Figure 3.10: Manufacturing of membrane-coated colloidal probe cantilevers. A small amount of molten epoxy resin is placed on the front end of a tipless cantilever, which is then brought into contact with a colloid of a defined size (A). After curing the epoxy glue, the colloidal probe cantilever is incubated with a suspension of small unilamellar vesicles $(B)$ in order to form a solid-supported membrane on the colloidal probe (C).

\footnotetext{
${ }^{44}$ Epoxy glue was a gift of Brenntag $\mathrm{GmbH}(1 \mathrm{~kg})$

A Force Spectroscopy Setup to Mimic Cellular Interaction Processes
} 
To simplify the attachment process a special microscope stage has been built, which allows heating up a confined area of the sample, the illumination of the attachment process through a cavity in this area, and the attachment of a nanomanipulator via magnets (Figure 3.11-B). The attachment process in detail is the following: freshly prepared epoxy resin is placed on a glass slide together with several milligrams of microspheres. The cantilever is attached to the holder using double-sided adhesive tape, inverted, and brought into proximity of the glass slide.
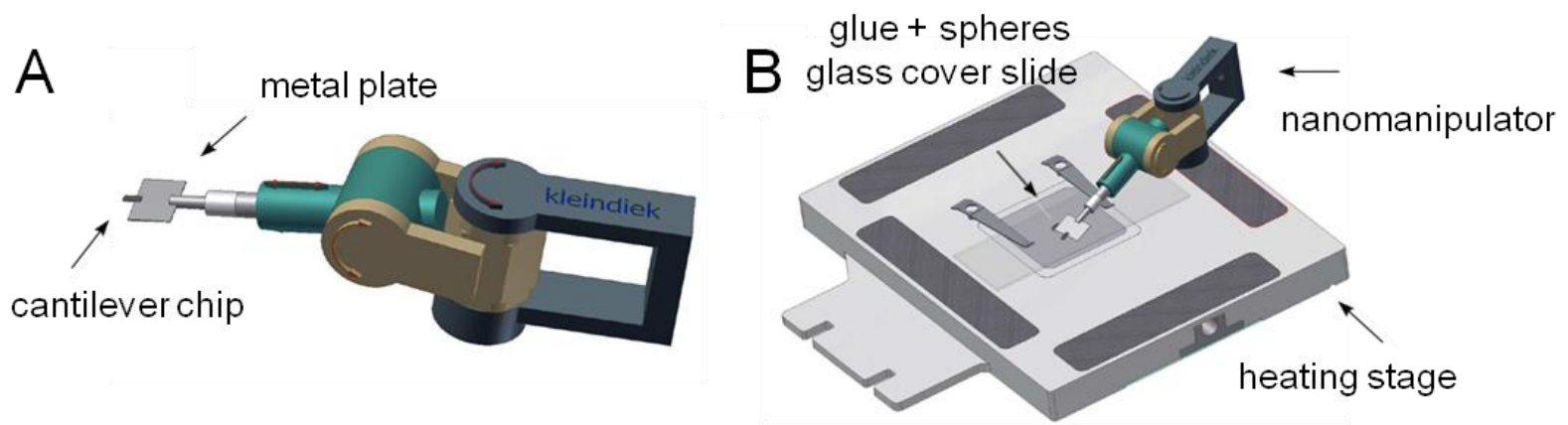

Figure 3.11: Nanomanipulator setup used to attach borosilicate colloidal spheres to tipless cantilevers. ${ }^{45} \mathrm{~A}$ Kleindiek MM3A-LS (A) is equipped with a small metal plate onto which a cantilever chip (small rectangular bar on the left side of the manipulator) is mounted. A home-built microscope stage (B) equipped with magnetic areas (dark grey) is used for correct positioning of the magnet-equipped manipulator relative to the epoxy resin and the spheres on the glass cover slide. A heatable area in the center of the stage is used for melting the epoxy resin used to attach the glass spheres. To avoid heating up of the microscope stage, a PTFE segment is used for thermal isolation of the stage. Graphics (created with "Autodesk Inventor").and manufacture of the stage by Volker Meyer, mechanics workshop, Institute of Physical Chemistry, Göttingen.

To produce a colloidal probe cantilever, the nanomanipulator is used to bring the front end of the cantilever into contact with a droplet of epoxy resin and with a microsphere within 10 seconds. A light microscope is used to visualize the process.

\subsubsection{Preparation of small unilamellar vesicles}

Depending on the phase behavior of the used lipid two techniques have been used to produce small unilamellar vesicles (SUVs): the extrusion method was used for the gel phase lipid DPPC (1,2-dipalmitoyl-sn-glycero-3-phosphocholine, Avanti Polar Lipids Inc., Alabaster, USA), whereas the sonication method was used for DOPC (1,2-dioleoyl-sn-glycero-3-phospho-

\footnotetext{
${ }^{45}$ Images kindly provided by Volker Meyer, fine mechanics workshop, University of Göttingen.
} 
choline) and POPC (1-palmitoyl-2-oleoyl-sn-glycero-3-phosphocholine, Avanti) vesicles. ${ }^{[121]}$ To form vesicles, lipid films were prepared from lipid stock solutions in $\mathrm{CHCl}_{3}$ by evaporating the solvent above the main phase transition temperature $T_{M}$. Films were hydrated with buffer to yield multilamellar vesicle (MLV) suspensions with a concentration of $1 \mathrm{mg}$ lipid/mL. Extrusion of DPPC vesicles was performed by using a mini-extruder (Avanti) with a single polycarbonate membrane with a pore diameter of $50 \mathrm{~nm}$ (Avestin, Mannheim, Germany). ${ }^{[121]}$ The vesicle suspension was passed through the membrane 31 times at $55{ }^{\circ} \mathrm{C}$. Sonication of fluid phase MLVs was performed in an ultrasonic homogenizer at RT for 2 times 20 min (Bandelin Sonopuls HD 2070, amplitude settings: $4 \times 10$ pulse, $70-75 \%$ intensity) to yield an SUV suspension.

\subsubsection{Preparation of membrane-coated probes and substrates}

Membrane functionalization of colloidal probe was performed using the vesicle spreading technique (Figure 3.10-B to -C). Prior to a force spectroscopy measurement, colloidal probe cantilevers were controlled via light microscopy and were cleaned in Argon plasma for $20 \mathrm{~s}$ (Harrick PDC-002, Ossining, NY, USA). A cantilever was mounted and incubated with a droplet of SUV suspension of a lipid concentration of 0.05 to $1 \mathrm{mg} / \mathrm{mL}$. After 15 minutes of incubation the cantilever was rinsed with $3 \mathrm{~mL}$ of buffer. The integrity of lipid membranes formed on the colloidal probe cantilever was controlled by means of fluorescence after photobleaching measurements (chapter 3.2).

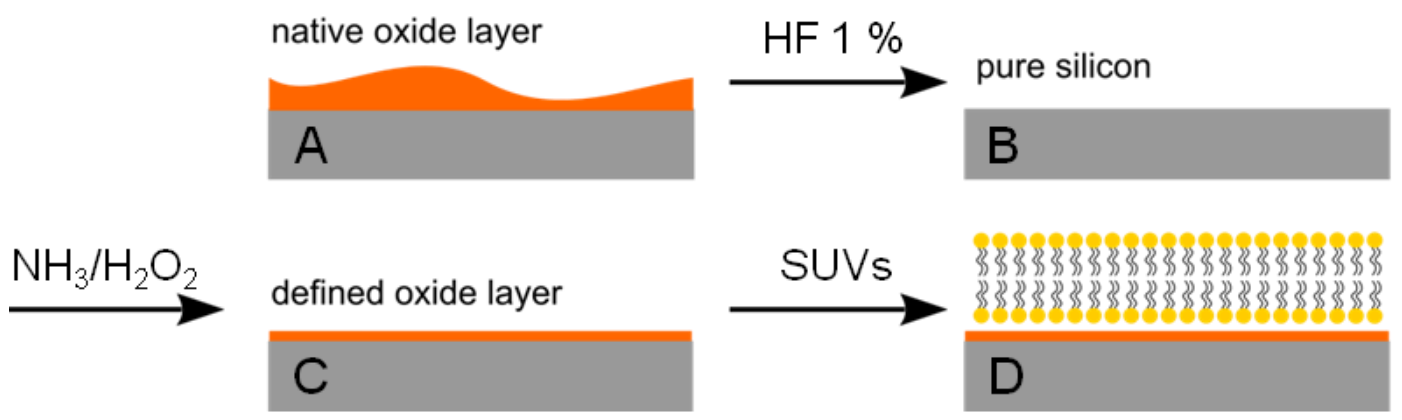

Figure 3.12: Membrane formation on silicon wafers. The native silicon oxide layer is reduced by HF treatment before the wafers are oxidized by $\mathrm{H}_{2} \mathrm{O}_{2} / \mathrm{NH}_{3}$ treatment to get a constant oxide layer thickness. Vesicle suspension is incubated on the wafer and excess vesicles are removed by thorough rinsing after the completion of the spreading process. 
Prior to spreading, silicon wafers (Active Business Company GmbH, Brunnthal, Germany; $525 \mathrm{~nm}$ thickness, $5.6-10.4 \Omega \mathrm{cm}$ ) were cleaned by rinsing them with isopropanol and deionized water. Removal of native $\mathrm{SiO}_{2}$ and controlled re-oxidation of the silicon wafer to an approximate oxide layer thickness of $3 \mathrm{~nm}$ was performed by using $1 \%$ hydrofluoric acid solution (15 min, at room temperature (RT)), and an aqueous solution of ammonia and hydrogen peroxide $\left(5: 1: 1 ; 15 \mathrm{~min}, 70^{\circ} \mathrm{C}\right)$, respectively (Figure 3.12). Wafers were rinsed thoroughly with deionized water and lipid bilayers were spread by incubating with a $0.1 \mathrm{mg} / \mathrm{mL}$ SUV suspension at RT for one hour. Subsequently, samples were extensively rinsed with buffer $(100 \mathrm{~mL})$ to ensure removal of excess vesicles. Gel phase lipid samples were incubated at $55{ }^{\circ} \mathrm{C}$ for 1 hour before rinsing. Prior to force spectroscopy measurements the integrity of the solidsupported lipid bilayers was controlled by imaging in contact mode (gel-phase membranes) or tapping mode (fluid-phase membranes).

\subsubsection{Membrane functionalization}

Solid supported lipid bilayers were functionalized with thiol-tagged molecules through conjugation of the free thiol group to a maleimide moiety on a fraction of phospholipids in a 1,2addition, which allows for controlling the number of molecules displayed on the membrane (Figure 3.13).

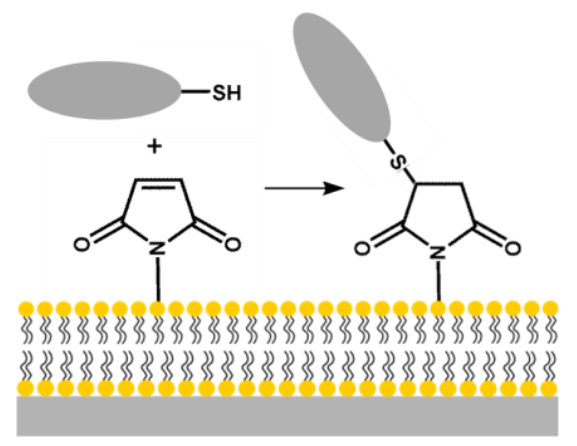

Figure 3.13: Covalent coupling of thiols to maleimide-bearing lipid membranes. Schuy et al. introduced this method for the in situ synthesis of lipopeptides on membrane surfaces in $2008 .{ }^{[94]}$

For gel phase bilayers, we used 1,2-dipalmitoyl-sn-glycero-3-phosphoethanol-amine-N-[4(p-maleimido-methyl)-cyclohexane-carboxamide] (MCC-DPPE), whereas for fluid phase membranes, we employed 1,2-dioleoyl-sn-glycero-3-phosphoethanol-amine-N-[4-(p-male- 
imidomethyl)cyclohexane-carboxamide (MCC-DOPE) in order to avoid phase separation due to chain mismatches.
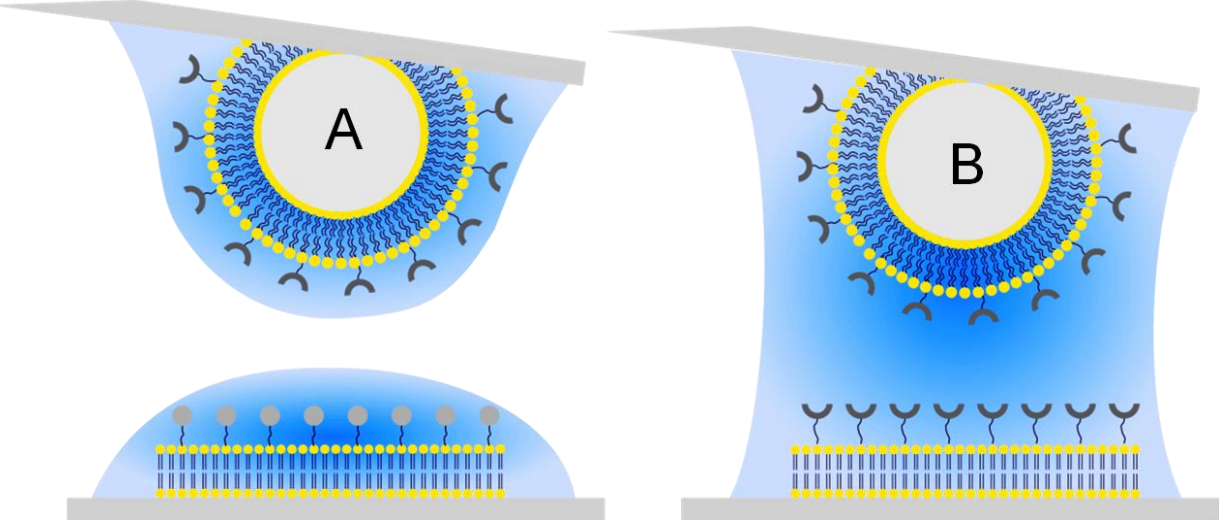

Figure 3.14: Homomeric and heteromeric incubation as employed in the different projects. For heteromeric interactions to be studied we incubated probe and sample in two different incubation chambers $(A)$. In the case of a homomeric interaction to be probed a simultaneous incubation technique was carried out in order to avoid the membranes to be exposed to a water/air interface (B).

Different types of incubation were used to functionalize maleimide-doped membranes. Heteromeric setups characterized by differing functionalization on probe and substrate membrane were treated as shown in Figure 3.14-A.

In contrast, for homomeric setups, which are equipped with two membranes displaying the same sort of receptor or ligand, a simultaneous incubation technique was employed (B). Details about the functionalization of membranes with peptides and disaccharides can be found in chapters 3.5.2 for the $\mathrm{Ni}^{2+}$-NTA-H6 interaction system, in chapter 3.6.2 for Microciona prolifera disaccharides, and in chapter 3.7.2 for the peptides $i$-E3Cys and $i$-K3Cys.

\subsubsection{Membrane probe spectroscopy measurements}

Force spectroscopy was used to determine interaction forces and surface adhesion energies between functionalized surfaces such as heteromeric and homomeric membrane setups (membrane probe spectroscopy, chapters 3.5.3, 3.6.3, and 3.7.3) and a homomeric protein setup (single-molecule force spectroscopy, chapter 3.8.3). 


\section{Force spectroscopy measurement}

To calibrate the membrane coated cantilever a thermal noise is recorded after a minimal equilibration time of five minutes and the probe is brought in proximity to the surface. Subsequently, an approach-retraction cycle is performed to create a repulsive contact with the sample at a trigger deflection of $100 \mathrm{~nm}$. Spring constant $k_{c}$ and inverted optical lever sensitivity (invOLS) values are obtained by fitting the thermal noise spectrum and the contact regime of the obtained curve and are used to convert photodiode voltage and piezo-distance into force-versus-distance data. Relevant parameters for force-distance measurements in this study are the approach and retraction speed $v$ (in $\mu \mathrm{m} / \mathrm{s}$ ), the load force on the surface $F_{\text {load }}$ (in $\mathrm{pN}$ ), the dwell time $t_{d}$ on the surface (in s), the sample rate used to record a force curve $r_{s}(\mathrm{~Hz})$, and the tip-sample separation $d$ (in $\mathrm{nm}$ ). In order to keep the trigger force on the surface constant during a set of experiments, it is recommendable to correct for drift during the approach of a cantilever by performing a linear fit operation along the baseline for each curve ("Virtual Line Deflection"). Figure 3.15 shows the scenario of a membrane-membrane interaction process as produced by a membrane probe force distance curve.
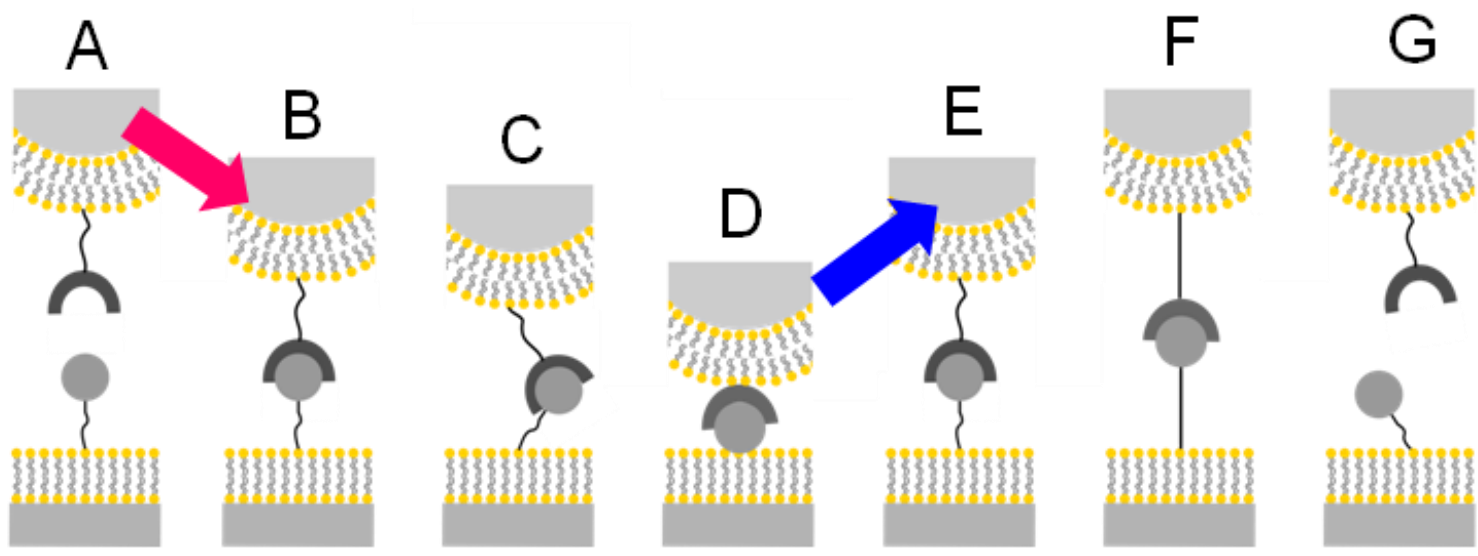

Figure 3.15: Complete cycle of a force curve with a membrane probe setup. Surfaces are coated with functionalized lipid membranes and brought in contact by moving the colloidal probe cantilever towards the substrate ( $A$ to $D)$. If a critical distance is reached, bond formation between ligands on probe membrane and receptors on substrate membrane is enabled ( $B$ to $F$ ). Upon retraction of the probe a release of the ligand-receptor bond is likely in the case of weak interactions (G), whereas in the case of nearly covalent interactions it is more probable that the system relaxes by sacrificing membrane material. 
The ligand-doped membrane probe cantilever is brought into contact with the receptordoped membrane on a planar substrate. A critical distance a ligand-receptor interaction is favored due to spatial and enthalpic reasons. If membranes are brought closer formed bonds are squeezed in between membranes until membranes are compressed with the trigger force mentioned above. During the dwell time ligand-receptor bond formation may be favorable in areas beyond the point of minimum distance. While retracting the membrane probe from the underlying membrane, it is conceivable that weak bonds tend to reopen and that strong bonds tend to hold until the membrane reservoir vanishes.

\section{Data analysis}

Raw data was processed by converting the deflection-piezo motion-curve into force-distance curves as described in chapters 3.1 and 3.1.2. We used Igor Pro equipped with MFP tools (Asylum Research, Santa Barbara, CA, USA) and a Matlab tool specially designed for the analysis of force spectroscopy data.

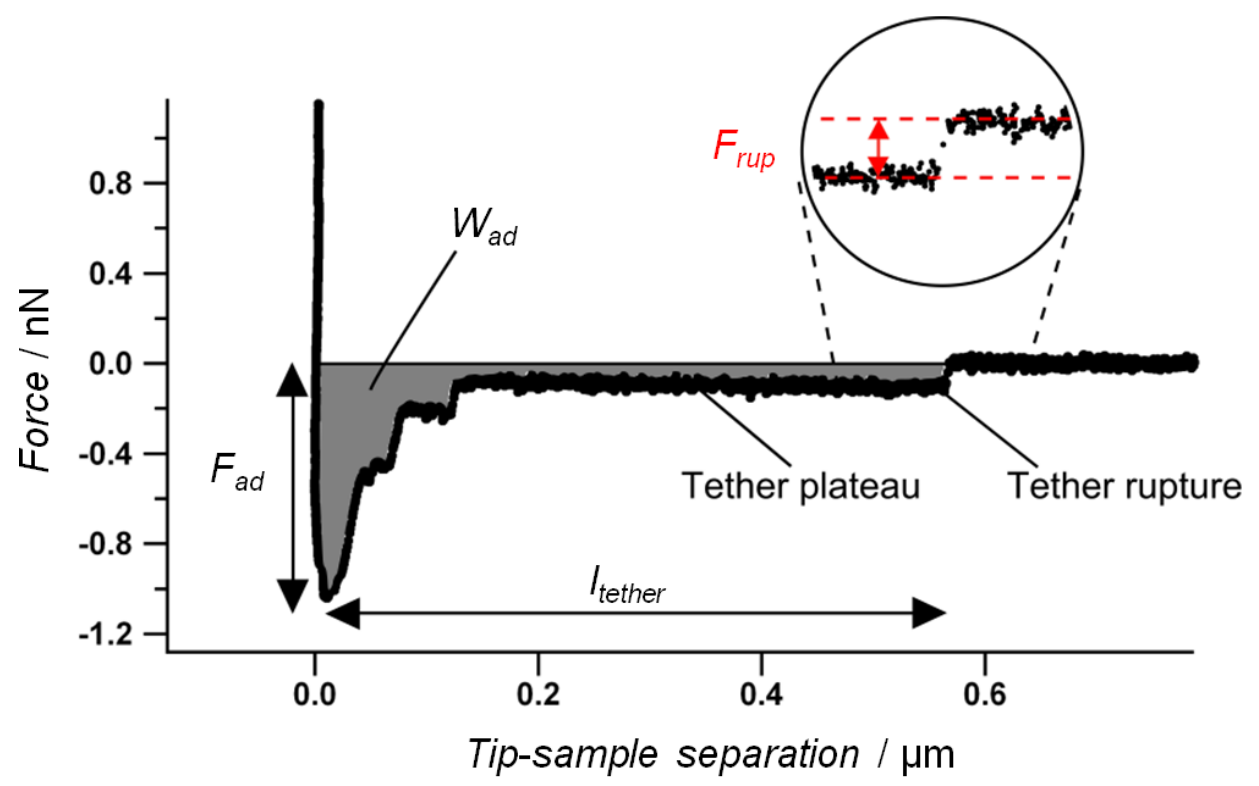

Figure 3.16: Typical force retraction curve as obtained after contacting a membrane functionalized with strong receptors (receptors: NiNTA, DOPC/Ni ${ }^{2+}$-DOGS-NTA (90:10) membrane) on a colloidal probe cantilever with a bilayer on a planar substrate, which is equipped with a ligand-functionalized membrane (ligands: His-tag peptides, on a maleimide-functionalized gel phase membrane (10 mol\% maleimide entities). ${ }^{[75], 46}$ Labels indicate the parameters that are generally obtained from the retraction curves: overall adhesion force $\boldsymbol{F}_{\text {ad, }}$, work of adhesion $\boldsymbol{W}_{\text {ad }}$ (gray area), number of tethers $\boldsymbol{N}_{\text {tethers, }}$ tether length $\boldsymbol{l}_{\text {tether }}$ and tether rupture forces $\boldsymbol{F}_{\text {tether }}$ are registered. This particular force curve has been recorded with a loading rate of approximately $2.5 \mathrm{nN} / \mathrm{s}$ and a dwell time on the surface of $1 \mathrm{~s}$.

\footnotetext{
${ }^{46}$ Image reprinted from [75] with the permission of Elsevier.
} 
Consequently, force-distance curves were analyzed for overall adhesion force $F_{a d ı}$ surface adhesion energy $W_{a d \text {, }}$ bond rupture events $F_{\text {rup, }}$ tether rupture events $F_{\text {tether, number }} N_{\text {tethers }}$ and length $l_{\text {tether }}$ of tethers as well as baseline noise (Figure 3.16). ${ }^{47}$ In our studies, we compared data extracted from measurements involving functionalized membranes to the data collected from non-functionalized ones.

\subsection{Membrane probe spectroscopy of the $\mathrm{Ni}^{2+}-\mathrm{NTA}-\mathrm{H} 6$ interaction}

\subsubsection{Synthesis and purification of $\mathrm{H6}$ peptides}

Ac- $\mathrm{H}_{6}$ GGC-NH $\mathrm{NH}_{2}(M=1100 \mathrm{~g} / \mathrm{mol}$, "H6", Figure 3.17) was synthesized via Merrifield solid phase synthesis with Fmoc chemistry, purified via ion exchange chromatography (IEC) and high performance liquid chromatography (HPLC), and subsequently characterized by matrixassisted laser desorption ionization - time of flight (MALDI-ToF) spectroscopy. ${ }^{[122]}$

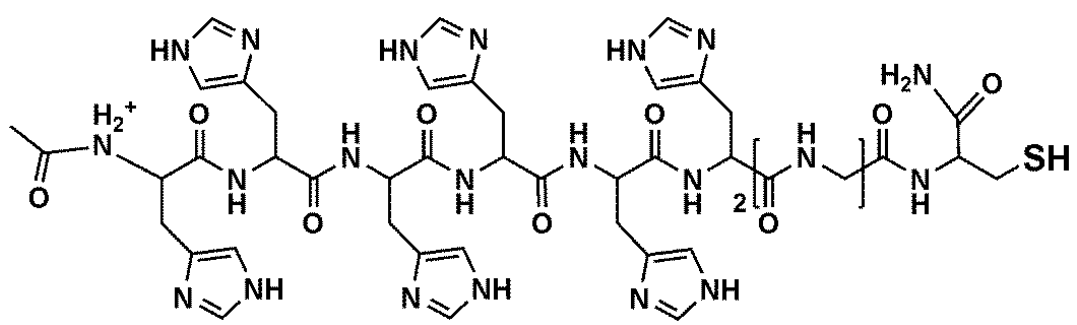

Figure 3.17: Chemical structure of $\mathrm{Ac}-\mathrm{H}_{6} \mathrm{GGC}-\mathrm{NH}_{2}$ (H6) as used for the functionalization of maleimidebearing membranes using 1,2-addition of thiol to maleimide moiety.

For the synthesis, we used N-a-Fmoc-a-S-amino acid derivates with standard side-chain protecting groups and coupling reagent 2-(1H-benzotriazole-1-yl)-1,1,3,3-tetramethyl-aminium hexafluorophosphate (HBTU) on a 4-methylbenzhydrylamine (MBHA) resin (Novabiochem, Darmstadt, Germany). Compounds were stored at $-18{ }^{\circ} \mathrm{C}$ after aliquotation into Eppendorf vials $(0.4 \mu \mathrm{mol}$ aliquots).

\footnotetext{
${ }^{47}$ Baseline noise was below $20 \mathrm{pN}$.
} 


\subsubsection{Functionalization of membranes with $\mathrm{Ni}^{2+}-\mathrm{NTA}$ and $\mathrm{H} 6$ residues}

\section{Preparation of membrane-coated silicon wafers and colloidal probes}

Silicon wafers were prepared for spreading as described in chapter 3.4.3. For all measurements, SUVs were prepared by extrusion using phosphate buffer ${ }^{48}\left(\mathrm{~PB} ; \mathrm{Na}_{2} \mathrm{HPO}_{4}, 50 \mathrm{mM}\right)$ at $\mathrm{pH} 5.9$ for lipid mixtures containing maleimide-functionalized vesicles, and at $\mathrm{pH} 6.8$ for $\mathrm{Ni}^{2+}$ NTA containing vesicles. SUVs were obtained by passing a suspension of MLVs 31 times through a single polycarbonate membrane (pore $\varnothing: 50 \mathrm{~nm}$, Avestin, Mannheim, Germany). Lipid bilayers were prepared by incubating the SUV suspension on silicon substrates at RT followed by incubation above the corresponding main phase transition temperature $\left(T_{M}\right)$ of the lipid, $\left(T_{M, \text { DOPC }}=-20^{\circ} \mathrm{C}, T_{M, D P P C}=41.5^{\circ} \mathrm{C} ; C_{\text {Lipid }}=0.1 \mathrm{mg} / \mathrm{mL}\right.$ of respective buffer, $30 \mathrm{~min}$ each step) and by thorough rinsing of the bilayer. For DOPC vesicles on the colloidal probe, incubation time was reduced to $15 \mathrm{~min}$ at RT. Bilayer formation and substrate coverage was controlled by epifluorescence microscopy and CLSM (probe) as well as by AFM imaging (wafer).

Lipid bilayers as spread on the colloidal probes were composed of DOPC, 1,2-dioleoyl-snglycero-3-[(N-(5-amino-1-carboxypentyl)imi-no-di-acetic acid) succinyl] (nickel salt) (DOGSNTA) and BY in a molar ratio of 89:10:1. For functionalization of planar silicon wafers, we used DPPC as a matrix lipid and MCC-DPPE in a 10 mol\% concentration as attachment site for Histag molecules. In the case of DOPC as lipid matrix, MCC-DOPE was used instead. Colloidal probe cantilevers were incubated with a DOPC/DOGS-NTA/BY (89:10:1) SUV suspension in PB 6.8 (1 mg lipid/mL). ${ }^{49}$ Vesicle sizes were determined by DLS (dynamic light scattering) measurements to range from 30 to $50 \mathrm{~nm} .{ }^{50}$ Silicon wafers were incubated with a $0.1 \mathrm{mg} / \mathrm{mL}$ DPPC/MCC-DPPE/BY (89:10:1) suspension in PB 5.9 and subsequently incubated with an excess of acetylated $\mathrm{H}_{6} \mathrm{GGC}-\mathrm{NH}_{2}(c=20 \mu \mathrm{M}, \mathrm{PB}$ 6.8) for 30 minutes followed by rinsing with PB 6.8.

\footnotetext{
${ }^{48} \mathrm{~A}$ list of buffers used in this work is given in chapter 8.5.

${ }^{49}$ A list of lipids and the main transition temperatures of matrix lipids is given in chapters 8.6.

${ }^{50}$ DLS measurements were performed by G. Pähler.
} 


\section{Functionalization of membranes with cysteine-tagged peptides $\mathrm{H} 6$}

Prior to incubation of membranes, peptides were dissolved in buffer by gentle vortexing. Maleimide-functionalized DPPC bilayers were incubated with acetylated $\mathrm{H}_{6} \mathrm{GGC}-\mathrm{NH}_{2}$ for 30 min at RT. After incubation, the samples were rinsed with PB 6.8 to remove excess of peptide structures in the supernatant.

\section{$\mathrm{Ni}^{2+}$-NTA-H6 interaction determined by complementary techniques}

Liposome spreading and binding processes of $\mathrm{H} 6$ peptides as well as vesicle fusion assays were performed in the working group and monitored by quartz crystal microbalance measurements in the dissipation mode (QCM-D) and Texas Red-self-quenching experiments, respectively.

\subsubsection{Membrane probe spectroscopy measurements with $\mathrm{Ni}^{2+}$-NTA-H6-doped membranes}

For force-distance measurements, we used triangular colloidal probe cantilevers with a nominal spring constant of $0.01 \mathrm{~N} / \mathrm{m}$ (chapter 3.4 .1 and chapter 5.1 ). ${ }^{51}$ Spring constants were calibrated as described above and were found to be approximately $0.03 \mathrm{~N} / \mathrm{m}$. During a measurement, the $\mathrm{Ni}^{2+}$-NTA-functionalized DOPC membrane probe was brought in contact with the H6-doped DPPC membrane on the planar support (setup shown in Figure 3.3, chapter 2.1).

Force-distance cycles were performed with a sample rate of $12.5 \mathrm{kHz}$, varying force load, dwell times, and pulling velocities. All measurements were performed in $50 \mathrm{mM} \mathrm{PB}(\mathrm{pH}=6.8$, RT) in a homemade PTFE measuring chamber. The integrity of lipid bilayers after forcedistance measurements was controlled by fluorescence microscopy.

\footnotetext{
${ }^{51}$ A list of cantilevers and their properties is given in chapter 8.4. 


\subsection{Membrane probe spectroscopy of (non-) sulfated disaccharides}

\subsubsection{Synthesis and purification of Microciona prolifera disaccharides}

Disaccharide structures were synthesized following the protocols of De Souza et al. ${ }^{52,[90,92]}$ Compounds were purified by column chromatography $\left(\mathrm{SiO}_{2}, \mathrm{CH}_{2} \mathrm{Cl}_{2} / \mathrm{MeOH}, 4: 1\right)$ affording 5.8 $\mathrm{mg}(10.4 \mu \mathrm{mol})$ of non-sulfated disaccharide and $5.3 \mathrm{mg}(8.03 \mu \mathrm{mol})$ of sulfated disaccharide as colorless oils (Figure 3.18). Characterization of compounds was realized by ESI-MS ${ }^{53},{ }^{1} \mathrm{H}$ NMR, and ${ }^{13} \mathrm{C}$ NMR measurements. ${ }^{54}$ Disaccharide structures were stored at $-20{ }^{\circ} \mathrm{C}$ after aliquotation into Eppendorf vials $(0.2 \mu \mathrm{mol}$ aliquots after dissolution in MilliQ water and vacuum centrifugation at RT).

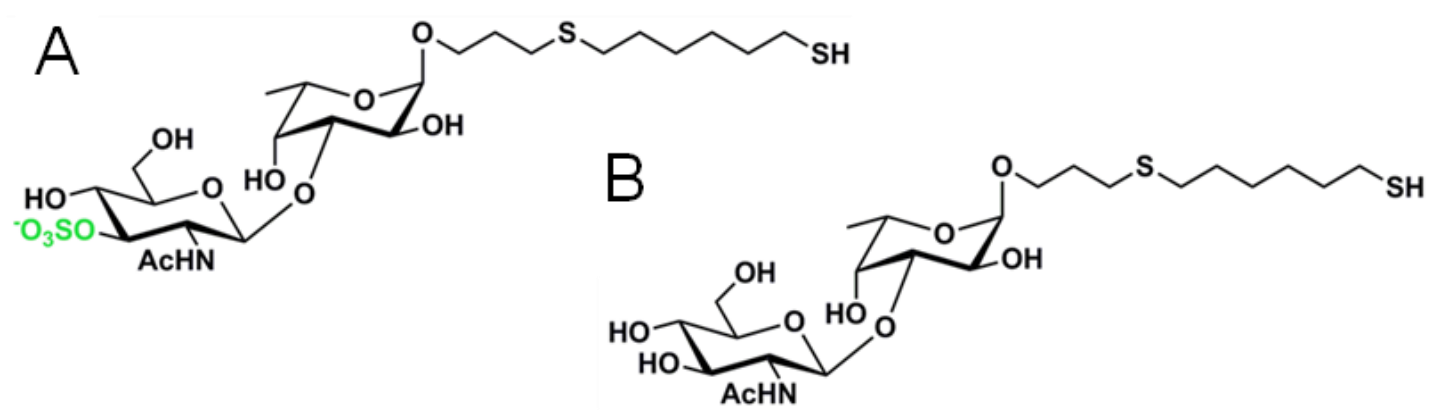

Figure 3.18: Chemical structures of non-sulfated (10-Mercapto-4-thia-dec-1-yl 2-Acetamido-2-deoxy- $\beta$-Dglucopyranosyl-(1 $\rightarrow 3)$ - $\alpha$-L-fucopyranoside) and sulfated (sodium salt of (10-Mercapto-4-thia-dec-1-yl-2acetamido-2-deoxy-3-O-sulfonato- $\beta$-D-glucopyranosyl-(1 $\rightarrow 3)$ - $\alpha$-L-fucopyranoside) thiol-linked disaccharides as used in the membrane probe spectroscopy setup [92],55 The sulfated epitope ( $\beta$-DGIcpNAc3S$(1 \rightarrow 3)$ - $\alpha$-L-Fucp) is reported to be the native self-recognition unit of the Microciona prolifera sponge. ${ }^{[90]}$ The non-sulfated compound is a synthetically modified structure, which is reported to be inactive in terms of self-recognition. ${ }^{[89]}$

\subsubsection{Functionalization of membranes with thiol-linked disaccharides}

\section{Preparation of membrane-coated silicon wafers and colloidal probes}

Prior to spreading, silicon wafers were cleaned with isopropanol and deionized, treated with $1 \%$ hydrofluoric acid (15 min, RT) to remove native $\mathrm{SiO}_{2}$, and an aqueous solution of ammo-

\footnotetext{
52 Synthesis and purification of compounds was carried out by Christian Brand and Ella Kriemen, group of Dr. D. B. Werz, Göttingen.

${ }^{53}$ A list of abbreviations can be found in chapter 8.1, page 126.

${ }^{54}$ Synthetic pathways of compounds, NMR spectra and chemical shift values are given in chapter 8.3.3.

${ }^{55}$ Images reprinted from [92] with the permission of the American Chemical Society.
} 
nia and hydrogen peroxide $\left(5: 1: 1,15 \mathrm{~min}, 70^{\circ} \mathrm{C}\right)$ to create an oxide layer of constant thickness. SUVs were prepared from DPPC, MCC-DPPE, and BY, in a molar ratio of 89:10:1. MLV suspension (PB 5.9, $c=1 \mathrm{mg} / \mathrm{mL}$ ) was transformed into SUVs by extrusion through a single polycarbonate membrane (pore diameter: $50 \mathrm{~nm}$, Avestin, Mannheim, Germany) 31 times at $55^{\circ} \mathrm{C}$. Before spreading of lipid membranes on the silicon wafers, they were rinsed with deionized water and dried in a stream of nitrogen. The vesicle suspension was incubated on the wafers overnight $(\mathrm{RT}, 0.1 \mathrm{mg} / \mathrm{mL})$ followed by one hour of incubation above the main phase transition temperature $\left(T_{M}\right)$ of the lipid $\left(T_{M, \text { DPPC }}=41^{\circ} \mathrm{C}\right)$. Samples were rinsed with PB 5.9, HEPES/EDTA-5 (50 mM HEPES, 5 mM EDTA, $\mathrm{pH}=7.4$ ), and HEPES/Ca ${ }^{2+}(50 \mathrm{mM}$ HEPES, 10 $\left.\mathrm{mM} \mathrm{Ca}{ }^{2+}, \mathrm{pH}=7.4\right)$ Successful bilayer formation was controlled by fluorescence microscopy and atomic force imaging (intermittent contact mode, MLCT-E (Bruker)). Spreading of membranes on colloidal probe cantilevers was performed following the procedure described in chapter 3.4.3. The probe was incubated with a $1 \mathrm{mg} / \mathrm{mL}$ POPC/MCC-DOPE/BY (89:10:1) SUV suspension, which was prepared by sonication. After an incubation time of $15 \mathrm{~min}$, the probe was rinsed with $\mathrm{HEPES} / \mathrm{Ca}^{2+}$ buffer.

\section{Functionalization of membranes with thiol-linked disaccharides}

Prior to incubation of membranes, compounds were dissolved in buffer by gentle vortexing. Maleimide-functionalized membranes on colloidal probe and silicon wafer were incubated simultaneously with non-sulfated or sulfated disaccharide (Figure 2.8 in chapter 2.2), respectively (70 $\mu \mathrm{M}, \mathrm{HEPES} / \mathrm{Ca}^{2+}$ buffer), for two hours at RT. After incubation, the samples were rinsed with HEPES $/ \mathrm{Ca}^{2+}$ buffer to remove excess of disaccharides in solution.

\section{Thiol binding as detected by reflectometric interference spectroscopy}

Silicon wafers with an oxide layer of five micrometer thickness were cleaned with an aqueous solution of ammonia and hydrogen peroxide $\left(5: 1: 1,70{ }^{\circ} \mathrm{C}, 15 \mathrm{~min}\right)$ and activated in oxygen plasma for $1 \mathrm{~min}$. After placing the sample in a flow cell, reflectivity spectra were recorded with a NanoCalc-2000-UV/Vis/NIR spectrometer in two second intervals. ${ }^{56}$

\footnotetext{
${ }^{56}$ RIfs measurements and data analysis were performed by Milena Stephan, University of Göttingen 
Small unilamellar vesicles prepared by sonication of a POPC/MCC-DOPE (90:10) MLV suspension (HEPES/Ca $\left.{ }^{2+}, \mathrm{pH}=5.9\right)$ were spread, rinsed with HEPES/EDTA-5 and sulfated Microciona prolifera disaccharide was added to the fluid circuit (100 $\mu \mathrm{M}$ in HEPES/EDTA buffer) and circulated for 60 min (Figure 3.19-A to -B).

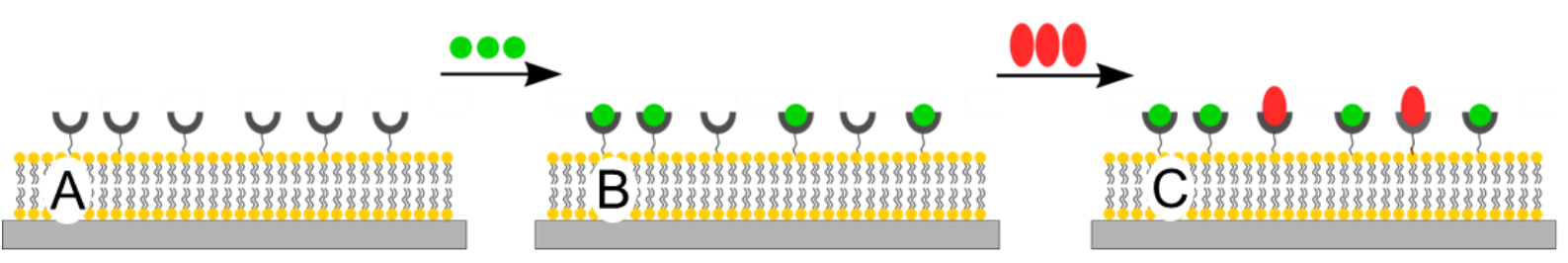

Figure 3.19: Experimental setup for the indirect detection of disaccharide thiol binding. First, a maleimide functionalized POPC bilayer is incubated with thiol-linked disaccharides (Step 1). Second, membranes are incubated with short cysteine-terminated peptides Ac- $\mathrm{H}_{6}$ WGC ("H6*", Step 2). During both steps changes in reflectivity of the surface are recorded. By analyzing the amount of bound peptides the amount of previously bound disaccharide thiols can be estimated.

To determine the number of free maleimide binding sites after covalent coupling of thiollinked disaccharides, an $\mathrm{Ac}-\mathrm{H}_{6} \mathrm{WGC}-\mathrm{NH}_{2}\left(\mathrm{H} 6^{*}\right)$ solution was added (200 $\mu \mathrm{M}, 40$ min) (Figure 3.19-B to $-C)$. Before each addition, the system was rinsed until the reflectivity signal was constant. Data was analyzed with a Matlab tool developed for this purpose. Each spectrum was fitted with a Matlab tool specially designed for the determination of the time-dependent optical thickness OT.

\subsubsection{Membrane probe spectroscopy measurements with (non-) sulfated di- saccharides-functionalized membranes}

Spring constants of colloidal probe cantilever were determined with the thermal noise method and found to be $k_{c}=(0.01 \pm 0.005) \mathrm{N} / \mathrm{m}$. Force distance cycles were performed with sample rates of $12.5 \mathrm{kHz}$, a force load in contact of $200 \mathrm{pN}$, a dwell time of $1 \mathrm{~s}$, and pulling velocities varying from $100 \mathrm{~nm} / \mathrm{s}$ to $5000 \mathrm{~nm} / \mathrm{s}$. Measurements were performed in a PTFE measuring chamber at RT in either HEPES/Ca ${ }^{2+}$ or in HEPES/EDTA-5 buffer. The integrity of the lipid membranes after the measurements was controlled with a fluorescence microscope. ${ }^{57}$

\footnotetext{
${ }^{57}$ Measurements were performed by Marieelen Oelkers during her diploma thesis supervised by B. L. 


\subsection{Membrane probe spectroscopy of the $i$-E3Cys/i-K3Cys interaction}

\subsubsection{Synthesis and purification of the peptides $i$-E3Cys and $i$-K3Cys}

The synthesis of coiled-coil peptides depicted in Figure 2.9 was carried out by Gesa Pähler. ${ }^{[95,}$ ${ }^{96]}$ Peptides $i$-E3Cys (Ac-(KELAAIE) ${ }_{3}$-GWGGGC-NH ${ }_{2}$ ) and $i$-K3Cys (Ac-WG(EKLAAIK) ${ }_{3}$-GGGGC$\mathrm{NH}_{2}$ ) were synthesized manually using Fmoc-protected $\alpha$-amino acids. ${ }^{[123]}$ Peptides were amidated at the $\mathrm{C}$-terminus and acetylated at the $\mathrm{N}$-terminus, cleaved from the MBHA resin using TFA and purified by RP-HPLC (L-6200A Intelligent Pump and L-4200 UV/Vis Detector, Merck-Hitachi, Darmstadt, Germany). For that purpose, Grace Vydac C18 columns and a linear gradient (solvent $\mathrm{A}: \mathrm{H}_{2} \mathrm{O} / \mathrm{AcCN} / T F A$, 99:1:0.1; solvent $\mathrm{B}: \mathrm{H}_{2} \mathrm{O} / \mathrm{AcCN} /$ TFA 10:90:0.05) were used. Identification of peptides was achieved via ESI-MS analysis (Apex IV, Bruker Daltonics, Billerica, MA, USA). The purity determined by RP-HPLC of resulting peptides was $94 \%$ for $i$ E3Cys and $88 \%$ for $i-K 3$ Cys. ${ }^{58}$

\subsubsection{Functionalization of membranes with $i$-E3Cys and $i$-K3Cys}

\section{Preparation of membrane-coated silicon wafers and colloidal probes}

Silicon wafers were prepared for spreading as described in chapter 3.4.3. For all measurements, SUV were prepared by sonication using PB $5.9(2 \times 20 \mathrm{~min})$. Bilayers were prepared from POPC SUV with a $T_{M}$ of $-2{ }^{\circ} \mathrm{C}$. Incubation at RT was sufficient to spread bilayers on colloidal probe ( $c_{\text {Lipid }}=1 \mathrm{mg} / \mathrm{mL}$ PB 5.9, 15 min incubation time) and silicon wafer ( $c_{\text {Lipid }}=0.1$ $\mathrm{mg} / \mathrm{mL}$ PB 5.9, $1 \mathrm{~h}$ incubation time). Lipid compositions were POPC/MCC-DOPE/BY on probe and wafer with a maleimide-lipid concentration of 0.1 to $10 \mathrm{~mol} \%$ and a BY concentration of 0.5 or $1 \mathrm{~mol} \%$.

\footnotetext{
${ }^{58}$ More details about peptide synthesis and purification can be found in 95. Pähler, G., et al. (2012). "Coiled-Coil Formation on Lipid Bilayers Implications for Docking and Fusion Efficiency." Biophysical Journal 103(11): 22952303.
} 


\section{Functionalization of membranes with $i$-E3Cys and $i$-K3Cys}

Membranes were rinsed with PB 6.8 and incubated with $i$-K3Cys (colloidal probe, 15 min, $\sim 100 \mu \mathrm{M}, \mathrm{PB} 6.8$ ) or with $i$-E3Cys (silicon wafer, $2 \mathrm{~h}, \sim 30 \mu \mathrm{M}$ ), respectively. Ellipsometry was used to detect the binding of Cys-tagged $i$-E3Cys to maleimide moieties of MCC-DOPE structures embedded in POPC membranes. The interaction of $i$-E3Cys with $i$-K3Cys was quantified by adding $i$-K3Cys-labeled SUV. Vesicle labeling was carried out by incubating MCC-DOPE labeled POPC vesicles (97:3) with $20 \mu \mathrm{M}$ i-K3Cys in PB 6.8 buffer and by removing unbound peptide by a size exclusion chromatography using Sephadex NAP-25 columns (illustra, GE Healthcare, Solingen, Germany). For ellipsometry measurements, silicon wafers were prepared following the protocol given in chapter 3.4.3. The measurement was performed in a closed fluid chamber (flow rate: $1.2 \mathrm{~mL} / \mathrm{min}$ ) part of an EP3-SW (Accurion, Göttingen, Germany) working at a wavelength of $532 \mathrm{~nm}$. After SUV spreading, the system was rinsed and $i$-E3Cys $(100 \mu \mathrm{M})$ was circulated through the flow chamber for 3 hours. When the angles del and psi were constant, $i$-K3Cys vesicles $(1.2 \mathrm{~mL})$ were added after rinsing. After reaching equilibrium, unbound vesicles were removed by flushing the system with buffer. Del and psi values were determined in real time using one zone measurements every $10 \mathrm{~s}$. Four zone measurements were carried out after completion of each incubation or rinsing step to obtain more accurate values.

\subsubsection{Membrane probe spectroscopy measurements with $i$-E3Cys/i-K3Cys- doped membranes}

Spring constants of the colloidal probe cantilever were determined with the thermal noise method and found to be $k_{c}=(0.01 \pm 0.005) \mathrm{N} / \mathrm{m}$. Force distance cycles were performed with sample rates of $12.5 \mathrm{kHz}$, a force load in contact of $200 \mathrm{pN}$, a dwell time of 1 second, and pulling velocities varying from $100 \mathrm{~nm} / \mathrm{s}$ to $5000 \mathrm{~nm} / \mathrm{s}$. Measurements were performed in a PTFE measuring chamber at RT in PB 6.8. The integrity of the lipid membranes after the measurements was controlled with a fluorescence microscope. 


\subsection{Single molecule force spectroscopy of cadherin interactions}

\subsubsection{Expression and purification of SNAP-tagged proteins EC15 and EC12}

Cadherin fusion proteins were expressed in stably transfected HEK 293 (Human Embryonic Kidney) cells..$^{59}$ Due to the lack of transmembrane domain, the proteins are delivered into the cell medium that is collected and treated with protease inhibitors.

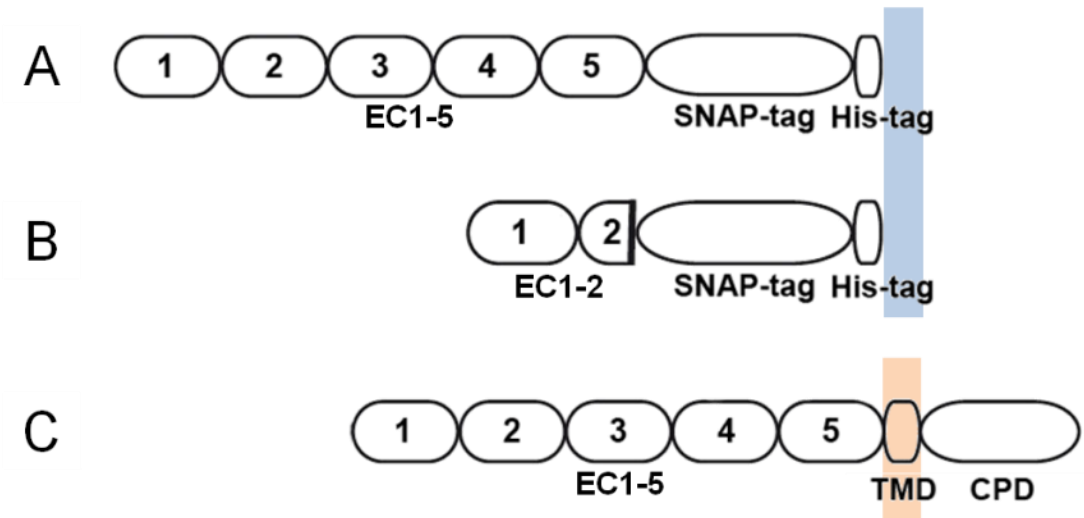

Figure 3.20: Schematic structures of the $120 \mathrm{kDa}$ protein construct EC15 (A) and the $55 \mathrm{kDa}$ construct EC12 (B) possessing a His-tag structure for their purification via affinity chromatography and a SNAP-tag to functionally couple them to benzylguanine-modified substrates. ${ }^{60}$ In contrast to the native structure depicted in C, the EC12 protein only possesses two subunits EC1 and EC2 of which EC2 is truncated. EC15 carries the native ectodomain consisting of subunits EC1-5. The native structure is characterized by a transmembrane domain (TMD) and a cytoplasmic domain (CPD). The orange and blue bars demonstrate the membrane or surface anchorage points of the structures.

After concentration of the supernatant proteins were purified by metal affinity chromatography on a $\mathrm{Ni}^{2+}-\mathrm{NTA}$ column. Eluted fractions were analyzed by SDS-PAGE and Western blotting. ${ }^{61}$ Protein concentrations were determined by using a BCA-assay kit and stored at $-80{ }^{\circ} \mathrm{C}$.

\section{SNAP-tag hAGT}

The SNAP tag is a mutant of hAGT, the human $\mathrm{O}^{6}$ alkylguanine DNA alkyl transferase, and is used for the site-specific immobilization of proteins and is derived from the DNA methylation machinery. ${ }^{[27,125]}$

\footnotetext{
${ }^{59}$ Protein expression and purification was carried out by Dr. Sinem Engin and Dagmar Fichtner, KIT Karlsruhe, Wedlich group

${ }^{60}$ Graphic taken from 124. Engin, S. (2010). Funktionalisierung strukturierter Oberflächen mit E-CadherinEktodomänen. Fakultät für Chemie und Biowissenschaften. Karlsruhe, Karlsruher Institut für Technologie (KIT)., with permission of S. Engin.

${ }^{61}$ More details about the Western blot analysis can be found in chapter 8.3.2.
} 
The enzyme hAGT catalyzes the transfer of alkyl groups from $\mathrm{O}^{6}$-alkylguanin-DNA to one of its cysteine residues. The SNAP tag mutant recognizes benzylguanine residues in a specific manner and is used in biotechnical applications in vivo as well as in vitro, i.e. for the protein expression in cells and for surface functionalization purposes. Its binding is specific, which ensures the accessibility of binding sites of a protein after being coupled. Furthermore, a variety of benzylguanine (BG)-modified compounds is available to which the SNAP-tagged proteins can be coupled to. ${ }^{[04]}$

\section{EC15 and EC12}

EC15 (hECad-EC1-5-SNAP-12His, Figure 3.20-A and Figure 2.15-A) and EC12 (hECad-EC1-2SNAP-12His, Figure 3.20-B and Figure 2.15-B) are proteins derived from the native E-cadherin ectodomain sequence (Figure 3.20-C), which originally consists of signal peptide, propeptide, ectodomains 1 to 5 (EC1 to EC5), a transmembrane domain (TMD) and a cytoplasmic domain (CPD) (Figure 3.20-C). The extracellular domain with their subunits EC1 to EC5 is responsible for the $\mathrm{Ca}^{2+}$-dependent interaction with the ectodomains of cadherin structures on neighboring cells. EC1 is the most distal subunit to the membrane and EC5 is the most proximal subunit to the membrane. By binding $\mathrm{Ca}^{2+}$ ions the ectodomain undergoes a transition from its coiled state to an elongated state as depicted in Figure 2.12, chapter 2.4, which enables the molecules to build intercellular interactions sites with other cadherin monomers.

\subsubsection{Functionalization of gold substrates with EC15 and EC12 proteins}

\section{Preparation of benzylguanine-thiol functionalized gold cantilevers and substrates} In order to prepare homogeneously thiol-coated gold substrates cantilevers (Bio-lever, BLRC150VB, Au coated from both sides, Olympus, Hamburg) and gold-coated glass cover slides (P231.1, $\varnothing=12 \mathrm{~mm}$, Carl Roth, Karlsruhe, Germany, $150 \mathrm{~nm}$ gold on $20 \mathrm{~nm}$ chromium) were cleaned in argon plasma for $20 \mathrm{~s}$ and subsequently functionalized by immersion in mixtures of receptor (BGT, benzylguanine -modified thiol, New England Biolabs, Frankfurt, Germany) thiol and methoxy-terminated thiol (matrix thiol, MT) of a concentration of 1:100 ( $c_{\text {thiol }}=$ $100 \mu \mathrm{M}$ in isopropanol) for $16 \mathrm{~h}$ (substrates) or $3 \mathrm{~h}$ (cantilevers) (Figure 3.21). 
MT<smiles>COCC(C)(C)COC(C)(CCCCCCCCCCCS)OC</smiles>

BGT<smiles>CCCCCCCCCCCCCCCCCCCOC(C)(C)CCC(C)(C)C(=O)NCc1ccc(COc2ncnc3[nH]cnc23)cc1</smiles>

Figure 3.21: Alkane thiols used to functionalize gold-coated substrates with a self-assembled monolayer (SAM) in order to covalently couple SNAP-tagged proteins. ${ }^{[104], 62}$ Methoxy-terminated EG3 thiol is used as a matrix thiol (MT) in combination with benzylguanine-modified thiol BGT, which serves as a receptor thiol for SNAP-tagged proteins.

Prior to functionalization with cadherin molecules, cantilever and substrate were rinsed with pure solvent and with HBS/EDTA-1 buffer (HEPES buffered saline supplied with ethylene diamine tetraacetic acid) to remove excess thiol from the solution. ${ }^{63}$

For micropatterned surfaces the procedure was the following: PDMS stamps were incubated with $40 \mu \mathrm{l}$ of thiol mixture (BGT:MT (1:100), $100 \mu \mathrm{M}$ ) for 5 min (Figure 3.22-A), pressed on the gold-coated cover slide (Figure 3.22-B), and removed after 2 min.
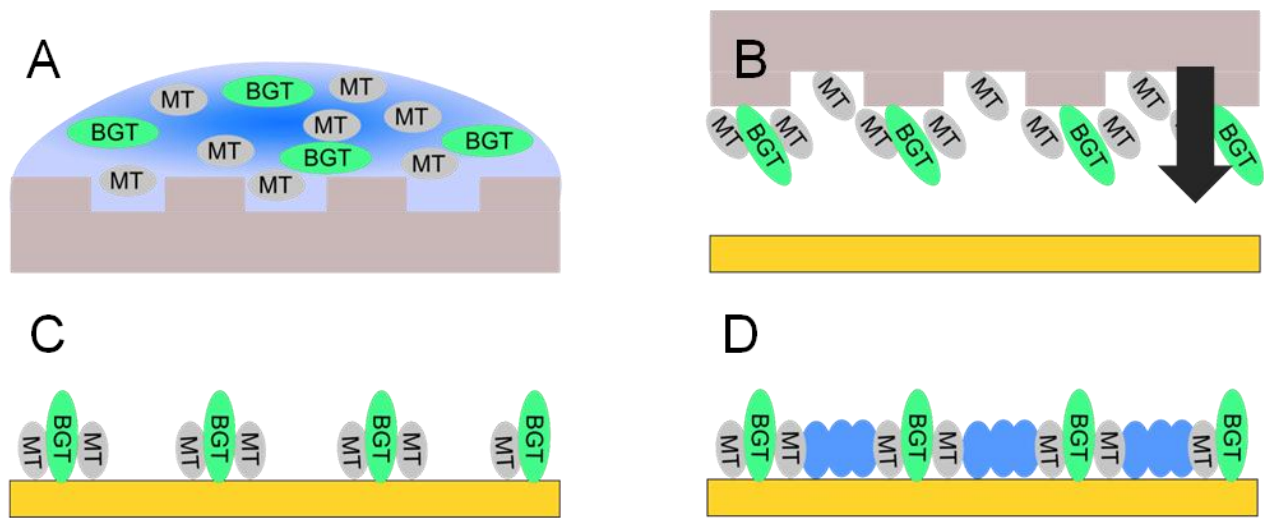

Figure 3.22: Microcontact printing $(\mu \mathrm{CP})$ technique as introduced by Whitesides and coworkers and employed by Engin et al. to prepare microstructured surfaces for the immobilization of SNAP-tagged Ecadherin structures. ${ }^{64,[104,127]}$ A PDMS stamp produced on a silicon master wafer is incubated with a thiol mixture (A) containing BGT and MT. After stamping the mixture on a clean gold surface (B to C), the thiolfree areas between the stamped structures are filled with pure $\mathrm{EG}_{4}$ thiol (D).

Excess thiol solution is removed by rinsing with isopropanol to give BGT:MT-functionalized surface (Figure $3.22-\mathrm{C}) . \mathrm{EG}_{4}$ thiol $(100 \mu \mathrm{M})$ was used to back-fill the thiol-free areas of the

\footnotetext{
${ }^{62}$ Image taken from [104] with the permission of the American Chemical Society.

${ }^{63}$ A list of buffers is given in chapter 8.5.

${ }^{64}$ Adapted from 126. Shin, H. (2007). "Fabrication methods of an engineered microenvironment for analysis of cell-biomaterial interactions." Biomaterials 28(2): 126-133.
} 
substrate as schematically shown in Figure 3.22-D (45 min of incubation time, followed by rinsing with isopropanol and drying in nitrogen stream). ${ }^{[29]}$

\section{Functionalization of thiol surfaces with SNAP-tagged proteins}

Tip and substrate surfaces were simultaneously incubated with $E C 12$ or EC15 solution ( $c_{\text {protein }}$ $=1-2 \mu \mathrm{M}$ in HBS-EDTA-1) to functionalize them according to the following reaction:

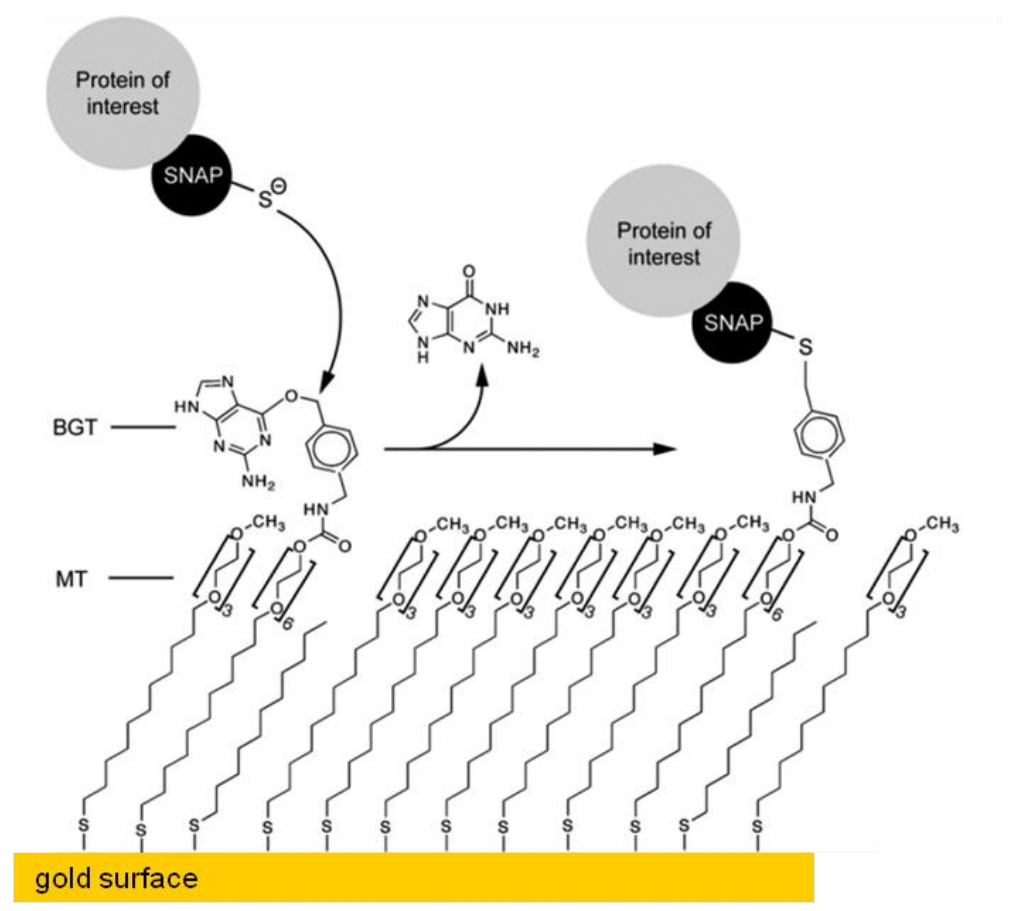

Figure 3.23: Immobilization of SNAP-tagged cadherin structures on a self-assembled thiol layer. ${ }^{65}$ The matrix thiols (MT) are mixed with BG-bearing thiol structures acting as receptors. By adjusting the ratio of receptor to MT, the amount of immobilized species can be controlled. Coupling of SNAP-tagged proteins to BG-functionalized surfaces has been introduced by Keppler in $2003 .{ }^{[27,128]}$ SNAP is flipping out BG in a $\mathrm{S}_{\mathrm{N}} 2$ mechanism reaction, which allows for site-specific immobilization of proteins. ${ }^{[125]}$

Prior to the force spectroscopy measurement, cadherin-coated surfaces were washed with $\mathrm{Ca}^{2+}$ buffer $\left(\mathrm{HBS} / \mathrm{Ca}^{2+}\right)$ and were activated by 30 min incubation in the same buffer.

\section{Protein binding as detected by secondary antibody staining of EC15 structures}

To control the site-specific immobilization of cadherin constructs secondary antibody staining has been employed.

\footnotetext{
${ }^{65}$ Adapted from 104. Engin, S., et al. (2010). "Benzylguanine Thiol Self-Assembled Monolayers for the Immobilization of SNAP-tag Proteins on Microcontact-Printed Surface Structures." Langmuir 26(9): 6097-6101. 
After incubation of surfaces with $1 \% \mathrm{w} / \mathrm{w}$ BSA (in phosphate buffered saline $\mathrm{w} / \mathrm{O} \mathrm{Mg}^{2+}$ and $\left.\mathrm{Ca}^{2+}\left(\mathrm{PBS}^{--}\right)\right)$, polyclonal primary antibodies $\alpha$-ECad $(\mathrm{H}-108$, rabbit; Santa Cruz Biotechnology Inc., CA, USA; 1:500) were coupled to EC15-functionalized surfaces ( $\left.1 \mathrm{~h}, 37^{\circ} \mathrm{C}\right)$ and subsequently incubated with fluorescently labeled secondary antibody $\mathrm{Cy}^{\mathrm{TM}} 3$-conjugated IgG (monoclonal, goat (anti-rabbit); Dianova, Hamburg, Germany, 1:100) after rinsing with phosphate buffered saline (PBS). Both incubation steps were carried out at $37^{\circ} \mathrm{C}$. Control samples were treated in the same manner skipping incubation with EC15.

3.8.3 Single molecule force spectroscopy of the homomeric cadherin interaction

\section{Homomeric interaction between EC15 or EC12 on gold substrates}

Force spectroscopy measurements were performed in the presence (Figure 3.24-A) and the absence of calcium ions (Figure 3.24-B). The reversibility of the calcium binding process was tested by switching from HBS/Ca ${ }^{2+}$ to HBS/EDTA-2 buffer and back to the original buffer. Buffer exchange was accomplished by thoroughly rinsing the system with $\sim 5 \mathrm{~mL}$ of buffer. In order to maintain protein functionality samples were always kept moisturized in buffer.

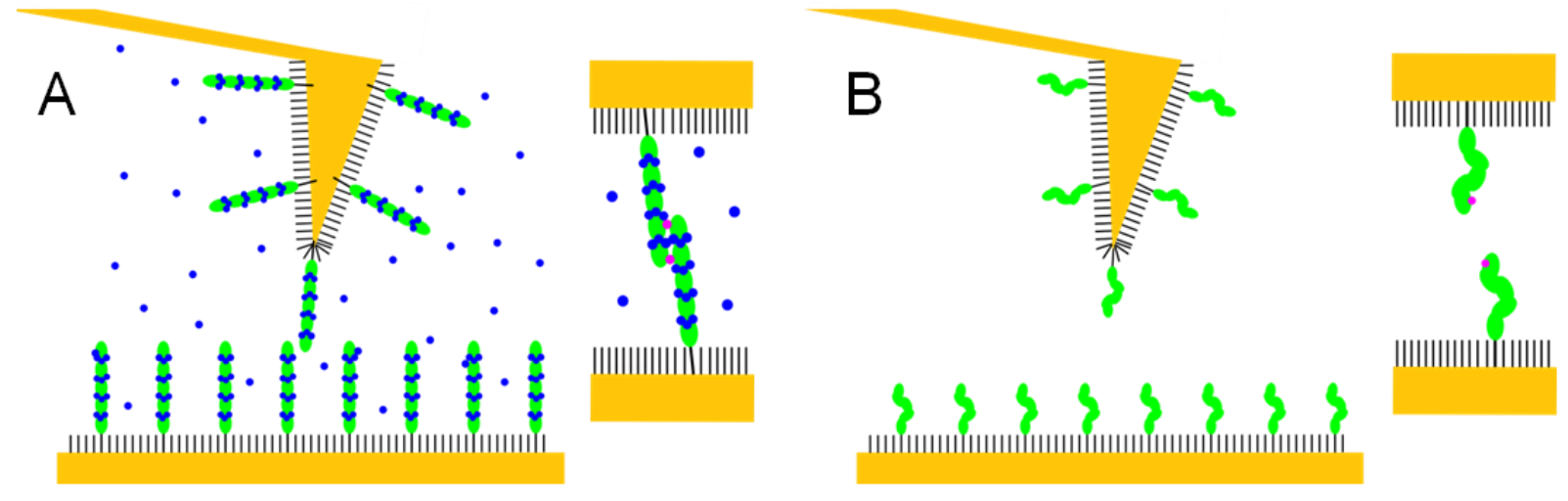

Figure 3.24: Schematic of the experimental setup used for the investigation of the homomeric cadherin interactions in the presence (A) and in the absence of calcium ions (blue spots) (B). Calcium ions are reported to be required for an efficient cadherin/cadherin interaction, which is characterized by the interaction of Trp2 residue (pink spots) on one cadherin ectodomain with the hydrophobic groove of a second ectodomain. 
After exchanging buffer to $\mathrm{Ca}^{2+}$ or EDTA buffer, the system was incubated for 30 min before the spectroscopy measurement was started. In all single molecule force spectroscopy experiments, the exact spring constant of the cantilever (nominal spring constant $k_{c}=6 \mathrm{pN} / \mathrm{nm}$ ) was determined by the thermal noise method prior to recording of curves. The force-distance curves were performed with a pulling velocity between 100 and $5000 \mathrm{~nm} / \mathrm{s}$ in HBS/EDTA-2 or $\mathrm{HBS} / \mathrm{Ca}^{2+}$ buffer at RT. Contact forces were in the range of 30 to $200 \mathrm{pN}$ and the contact time varied between 0 and $5 \mathrm{~s}$.

\section{Interaction between EC15 and NMuMG cells}

Cadherin -cell interactions between native NMuMG (normal murine mammary gland, mouse epithelium) cells were probed before and after cytokine TGF- $\beta$ treatment, which induces the epithelial-mesenchymal transition (EMT) of the cells resulting in changes of cell shape and cadherin expression. ${ }^{[129]}$ TGF- $\beta$ was used to down-regulate E-cadherin expression and to upregulated $\mathrm{N}$-cadherin (neuronal tissue cadherin) expression in order to probe $\mathrm{E}$-cadherin/Ecadherin interactions as well as E-cadherin- $\mathrm{N}$-cadherin interactions. For this purpose, cadherin-functionalized cantilevers were prepared in order to probe EC15 interactions (Figure 3.25) with NMuMG cells before and after incubation with TGF- $\beta$.

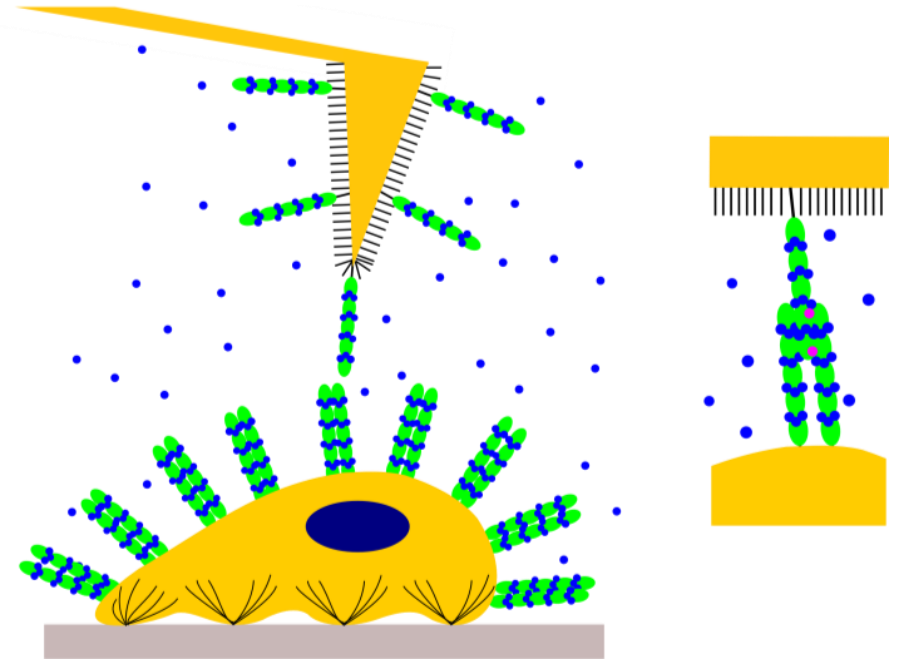

Figure 3.25: Schematic of the experimental setup as used to detect interactions between EC15 structures on an AFM tip and cells adhering to a Petri dish substrate. A lateral profile of forces produced by the interaction of cadherin molecules on the tip and the cell surface can be recorded by mapping the cell surface with the functionalized cantilever. As the height signal of the cantilever is recorded instantaneously, the results can be interpreted in terms of the spatial distribution of cadherin structures on the cell surface. 
Cells were obtained from the American Tissue Culture Collection (ATCC, Manassas, VA) and were maintained in Dulbecco's modified Eagles medium with $10 \%$ fetal calf serum, $10 \mu \mathrm{g} / \mathrm{mL}$ insuline, $4.5 \mathrm{~g} / \mathrm{l}$ glucose, and $2 \mathrm{mM} \mathrm{L-glutamine} \mathrm{(PAA} \mathrm{Laboratories} \mathrm{GmbH}$, Cölbe, Germany). They were seeded into a Petri dish $48 \mathrm{~h}$ before starting the spectroscopy measurements and grown to confluence. To ensure the complete EMT of cells, mesenchymal-like cells were incubated with TGF- $\beta 1$ for $48 \mathrm{~h}$.

To probe EC15-interactions we used Biolevers functionalized with BG/MT thiols (1:100) and EC15 proteins ( $1 \mu \mathrm{M}$, in HBS/EDTA-1) as described in chapter 3.8.2. with HBS/EDTA-1 buffer used for rinsing.

After immersing the cantilevers in serum-free medium (UltraCULTURE, w/o L-Gln, Thermo Fisher Scientific, Rockford, IL, USA; supplied with $200 \mu \mathrm{g} / \mathrm{ml}$ penicillin/streptomycin, $5 \mu \mathrm{g} / \mathrm{mL}$ amphotericin), the system was allowed to reach thermal equilibrium (10 min). Hereafter, the cantilever was brought in contact with an adherent NMuMG cell for $1 \mathrm{~s}$ (contact force: 50 $\mathrm{pN})$.

Force-distance cycles were carried out on TGF- $\beta$ treated and untreated cells while keeping the approach and retraction speed at $1 \mu \mathrm{m} / \mathrm{s}$, which corresponds to an approximate loading rate $R_{f}$ of $6 \mathrm{nN} / \mathrm{s}$. The maximum cantilever-surface distance between the measurements was 3 $\mu \mathrm{m}$. Calcium ion concentration in the UltraCULTURE medium is about $0.9 \mathrm{mg} / \mathrm{mL}^{66}$

\footnotetext{
${ }^{66}$ Information of Thermo Fisher Scientific customer service 


\section{Probing receptor-ligand interactions with an atomic force microscope}

Unbinding events are thermally activated processes with a statistical probability to occur. Rupture probability can be enhanced by applying external forces to the bonds as it is the case for force spectroscopy methods. The measured rupture forces $F_{\text {rup }}$ heavily depend on the force rate that is used for stretching the bond. In atomic force spectroscopy measurements, bonds are loaded with a force excerted by a cantilever of stiffness $k_{c}$, which changes the energy landscape of the interaction.

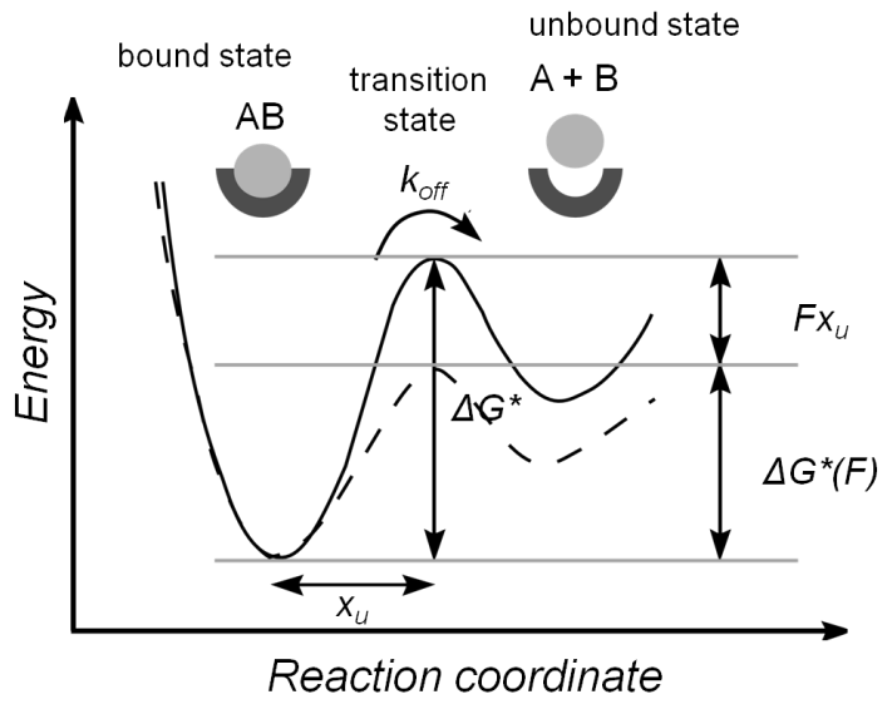

Figure 4.1: Energy profile of a receptor-ligand interaction process with and without an external force $F$ acting on the bond. ${ }^{67}$ The potential indicates a certain probability of bond dissociation given by the off rate $k_{o f f}$, the on rate $k_{o n}$, the potential width $x_{u}=x_{\beta}$ and the free energy of unbinding $\Delta G^{\circ}$. Upon application of an external force $F$ to the bond the free energy of unbinding $\Delta G(F)$ is reduced by the factor $F x_{\beta}$.

The term energy landscape refers the energy profile of an interaction between ligand $A$ and receptor $B$, which is depicted in Figure 4.1. In the simplest case, it is defined by two local minima equivalent to the bound state $A B$ and the unbound state $A+B$. Parameters characterizing the potential of a bond are on-rates and off-rates $k_{\text {on }}$ (unit: $\mathrm{M}^{-1} \mathrm{~s}^{-1}$ ) and $k_{\text {off }}$ (unit: $\mathrm{s}^{-1}$ ) also known as association and dissocation rates as well as the potential width $x_{u} \cdot(\mathrm{unit}: \mathrm{nm})$.

\footnotetext{
${ }^{67}$ Adapted from 65. Bizzarri, A. R. and Cannistraro, S. (2010). "The application of atomic force spectroscopy to the study of biological complexes undergoing a biorecognition process." Chemical Society Reviews 39(2): 734-749. 
Rates are defined by the speed of transition from unbound to bound state and vice versa The parameter $k_{\text {off }}(0)$ determines the lifetime $\tau$ of the bond in absence of a loading force $F$ and the parameter $x_{u}$ is characterized by the width of the potential of the bound state and the free energy of unbinding $\Delta G^{\circ}$.

Application of a force $F$ to a formed bond as depicted in Figure 4.2., $4 \rightarrow 5$ results in a stabilization of the unbound state as well as in a lowering of the transition state energy level. To cite Aleksandr Noy, "a force-induced bond rupture in force spectroscopy measurements is a thermally driven transition from the bound state into an unbound state over a potential energy surface that is constantly modified by the time-dependent potential of the loading spring. ${ }^{[130]}$ Referring to Figure 4.1, pulling on a bond equals a reduction of the activation energy barrier $\Delta G^{\circ}$ (now called $\Delta G^{\circ}(F)$ ) by $F x_{u}$ and results in a bond rupture as a result of thermal noise. Upon application of a force to a bond, an exponential increase in dissociation rates $k_{\text {off }}(F)$ and a promoted unbinding probability is observed. Moreover, measured bond rupture forces $F_{\text {rup }}$ depend on the rate $R_{f}$ a bond is loaded with.

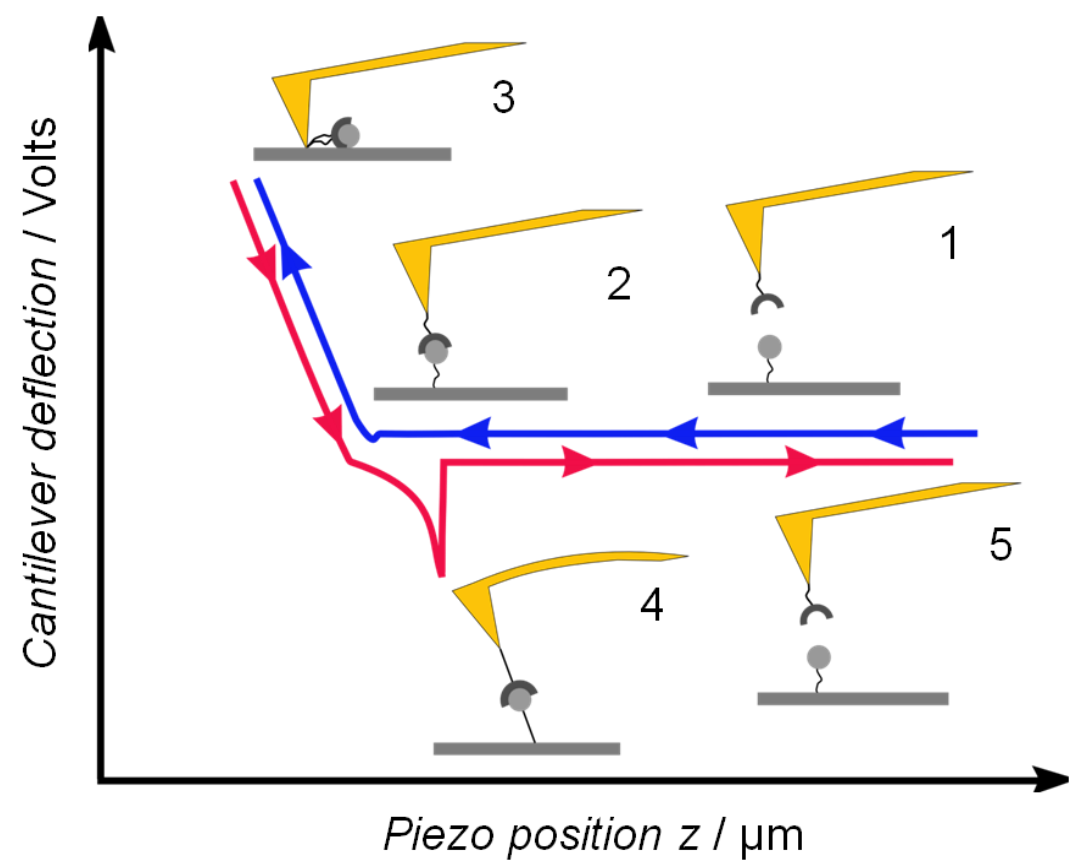

Figure 4.2: Probing a single biomolecular interaction in a single molecule force spectroscopy setup. ${ }^{67}$ No interaction between tip and surface is detected at positions 1 and 5 . Approaching of the surfaces leads to the formation of a bond between receptor-doped cantilever and ligand-doped substrate at a critical separation of the molecules (2). In repulsive contact with the surface, the cantilevers' upward deflection increases. When retracting the cantilever the bond formed between receptor and ligand is stretched leading to a downward deflection of the lever, which is acting like a loaded spring (4). After bond rupture the cantilever snaps back to its original position (5). 
This relation allows the determination of binding kinetics by performing loading ratedependent force spectroscopy measurements/dynamic force spectroscopy (DFS) as described by Evans (Equation 6). ${ }^{[131,132]}$

$$
k_{\text {off }}(F)=k_{\text {off }} \exp \left(\frac{x_{u} F}{k_{b} T}\right)
$$

Equation 6

It is striking that the dynamic force spectroscopy pull-off forces increase logarithmically with the loading rate $r_{f .}$. Dynamic force spectra corresponding to interaction potentials are given on the right side of Figure 4.3. A non-linearity in DFS is observed if complex potentials are involved, which exhibit two or more transition states.
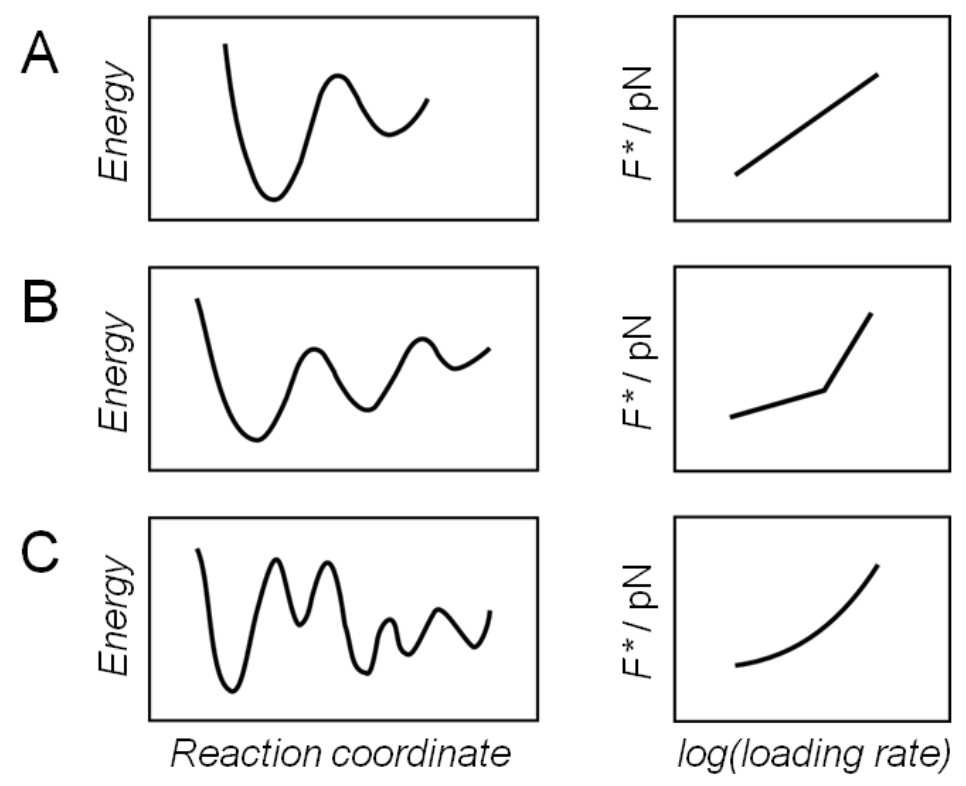

Figure 4.3: Possible interaction potentials of a biomolecular complex and the most probable rupture force $F^{*}$ as a function of the loading rate $R_{f}{ }^{67}$ A linear relation between $F^{*}$ and the logarithm of $R_{f}$ is observed in the case of an interaction with a single transition state. Two linear regimes of $F^{*}$ are observed in the case of the occurrence of bimolecular transition. A more complex behavior of $F^{*}$ is detected if a multimolecular interaction system is probed.

Depending on the applied loading rate, force spectroscopy measurements are defined as near-equilibrium measurements or non-equilibrium measurements. If loading rates are low compared to the experimental timescale, a bond system is considered to be close to its thermal equilibrium. 
In this case, rebinding has to be encountered. In contrast, when a bond is subjected to a high loading rate, the system is considered to be far from equilibrium and rebinding can be neglected. Evans and Ritchie solved the kinetic equations for this case and found that most probable binding force $F^{\star}$ depends on the loading rate according to the expression given in Equation 7

$$
F^{*}=\frac{k_{b} T}{x_{u}} \ln \left(\frac{x_{u}}{k_{b} T \cdot k_{\text {off }}}\right)+\frac{k_{b} T}{x_{u}} \ln \left(R_{f}\right)
$$

Equation 7

As depicted in Figure 4.2, probing bonds in a force spectroscopy setup causes stretching of the linker region of the interaction (state 4). In a physical point of view, the cantilever-bond system equals a stretching process of two elastic components defined by the bond stiffness $k_{s}$ and the stiffness $k_{c}$ of the cantilever. It has been shown that besides the stiffness $k_{s y s t e m}$ composed of $k_{s}$ and $k_{c}$ contributions; the linker length $l_{\text {linker }}$ has an impact on energy landscapes of a bond as well.

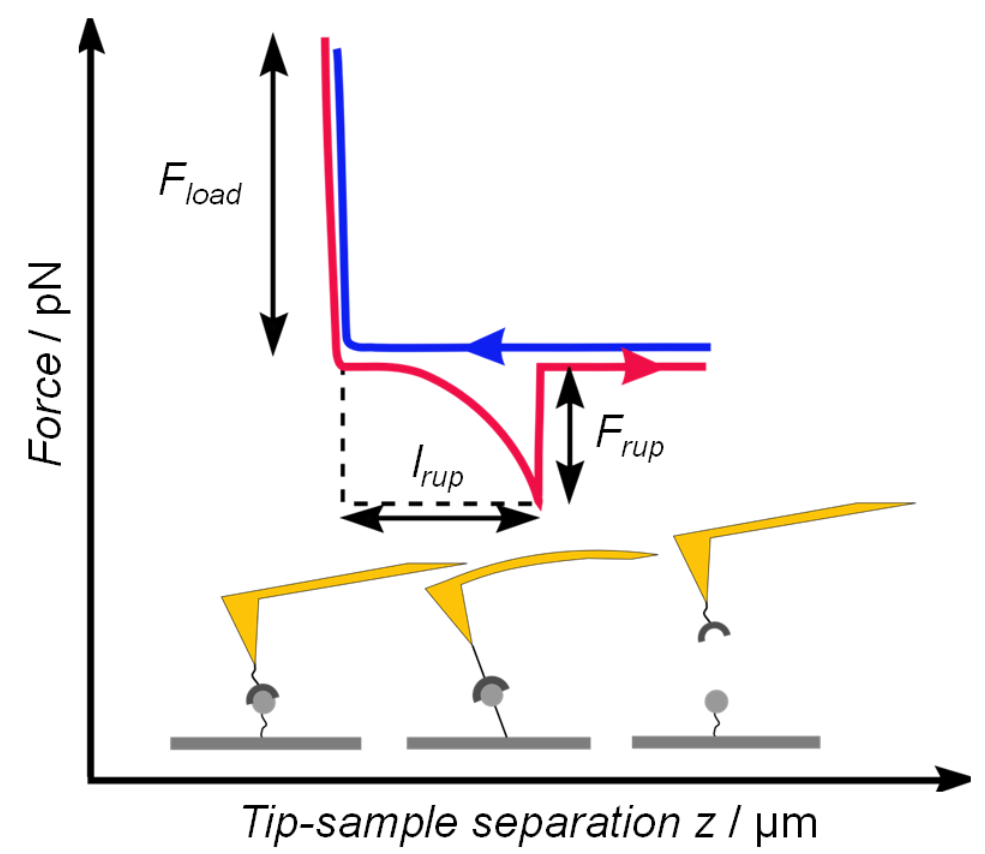

Figure 4.4: A force profile of a tethered ligand-receptor interaction. ${ }^{67}$ As the connection between probe and sample surface behaves like an elastic spring a worm-like chain like behavior of the bond is observed upon retraction of the cantilever. 
If flexible tethers such as proteins are being probed, one has to account for the entropic elasticity of the polymer tether representing a nonlinear spring in series with the chemical bond. The forces acting on the bond as well as the potential energy of the system will be altered in this case. Such a probe biopolymer exhibits a characteristic force profile in force-distance curves, which is a result of the elastic response of the polymer.

An initial soft region is followed by a stiffening of the molecule due to disentanglement of its coiled structure (Figure 4.4). The model used here to describe this behavior of a polymer, which is stiff on the scale of the persistence length $l_{p}$ is the worm-like chain model. Bustamante and coworkers introduced the following expression to describe the force extension of polymer as: ${ }^{[133]}$

$$
F(x)=\frac{k_{b} T}{l_{p}}\left[\frac{1}{4}\left(1-\frac{x}{l_{c}}\right)\right]^{-2}-\frac{1}{4}+\frac{x}{l_{c}}
$$

Equation 8

Where $F(x)$ is the force acting on the polymer upon extension by a distance $x$, and $l_{c}$ the contour length of the polymer (Figure 4.5) Soft linkers usually result in smaller rupture forces and broader distributions.

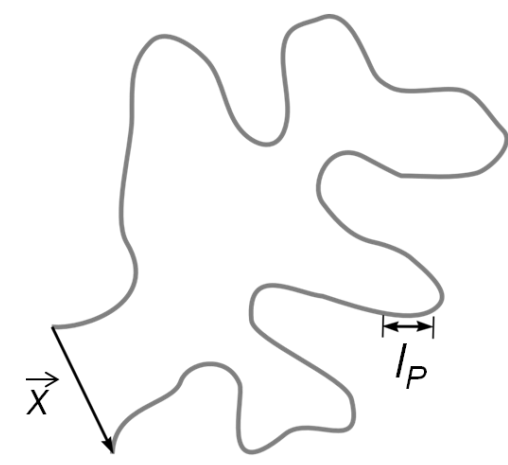

Figure 4.5: The worm-like chain model for the description of protein unfolding processes. ${ }^{68}$ The model describes the polymer as a continuously curved filament which is stiff on the scale of the persistence length $l_{P}$. In this study, the model is used to describe homomeric cadherin interaction processes.

Adapted from 109. Janshoff, A., et al. (2000). "Force Spectroscopy of Molecular Systems-Single Molecule Spectroscopy of Polymers and Biomolecules." Angewandte Chemie International Edition 39(18): 3212-3237. 
A Force Spectroscopy Setup to Mimic Cellular Interaction Processes 


\section{Membrane probe spectroscopy}

\subsection{Manufacturing of membrane probe cantilevers}

\subsubsection{Colloidal probe cantilevers}

Colloidal probe cantilevers were prepared as described in chapter 3.4.1. Figure 5.1-A shows the front end of a tip-less cantilever as used for the production of colloidal probe cantilever $(B, C)$. It is crucial to the functionality of the colloidal probe cantilever to use small amounts of resin and clean glass beads.

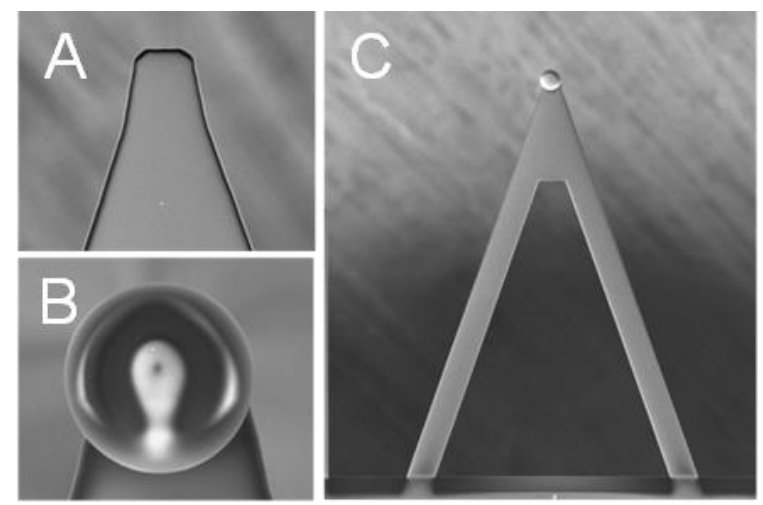

Figure 5.1: Scanning electron micrographs of cantilevers before and after attachment of a colloidal probe. ${ }^{69}$ A: front end of a tipless cantilever (MLCT-010, Bruker AFM Probes, Camarillo, CA, USA). ${ }^{70}$ B, C: Colloidal probe cantilever manufactured from tipless cantilever as shown in $A$ and borosilicate spheres with a diameter of $15 \mu \mathrm{m}$ : front end (B) and full length cantilever (C).

Correct positioning of the colloid at the front end of the cantilever is vital to the analyzability of spectroscopy data. Force spectroscopy measurements can only be performed if the colloid comes in contact with the surface before the front end of the cantilever touches the surface. If the colloidal probe is positioned at the very front end of the cantilever (Figure 5.2-1 and 2), one risks inducing undesirable upward bending of the cantilever during the contact time. This may lead to irregularities of the photodiode signal and to sliding of the colloidal probe on the surface resulting in inaccurate load forces, difficulties during data analysis, and noninterpretable data.

\footnotetext{
${ }^{69}$ Scanning electron microscopy (SEM) imaging performed by Julia Braunger, University of Göttingen, usage of images permitted.

${ }^{70} \mathrm{~A}$ list of cantilever and their characteristics can be found in chapter 8.4.
} 
If the probe is attached asymmetrically as depicted in Figure 5.2-6 and -7, a torsion of the cantilever during surface contact is most likely, which leads to difficulties during data analysis, as well.

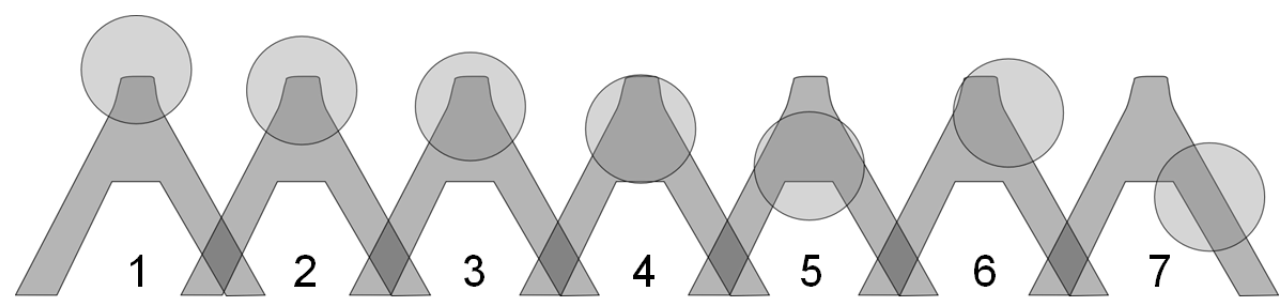

Figure 5.2: Correct positioning of glass beads on tipless cantilevers is a requirement for the production of colloidal probe cantilevers for force spectroscopy measurements. Positions 2, 3, and 4 are acceptable for a force spectroscopy measurement, whereas positions 1, 5, 6, and 7 are not as a measurement with those would result in an unpredictable deflection of the cantilever, which cannot be corrected after the measurement. Those cantilevers were excluded from force spectroscopy measurements.

To avoid that, only probes such as shown in Figure 5.2-3, -4, and -5 were used for force spectroscopy measurements. Due to the large probe diameter used in our experiments, we can exclude that the front end of the cantilever is in contact with the surface rather than the colloidal probe even though the cantilever is tilted towards the surface to an angle of $11^{\circ}$ during the experiment.

\subsubsection{Manufacturing of membrane probe cantilevers}

Membrane probe cantilevers were prepared as described in chapter 3.4.3. Successful functionalization of the colloidal probe was achieved by incubating the cantilever in a $1 \mathrm{mg} / \mathrm{mL}$ fluid phase lipid vesicle suspension for at least 5 minutes (standard incubation time: $15 \mathrm{~min}$ ).
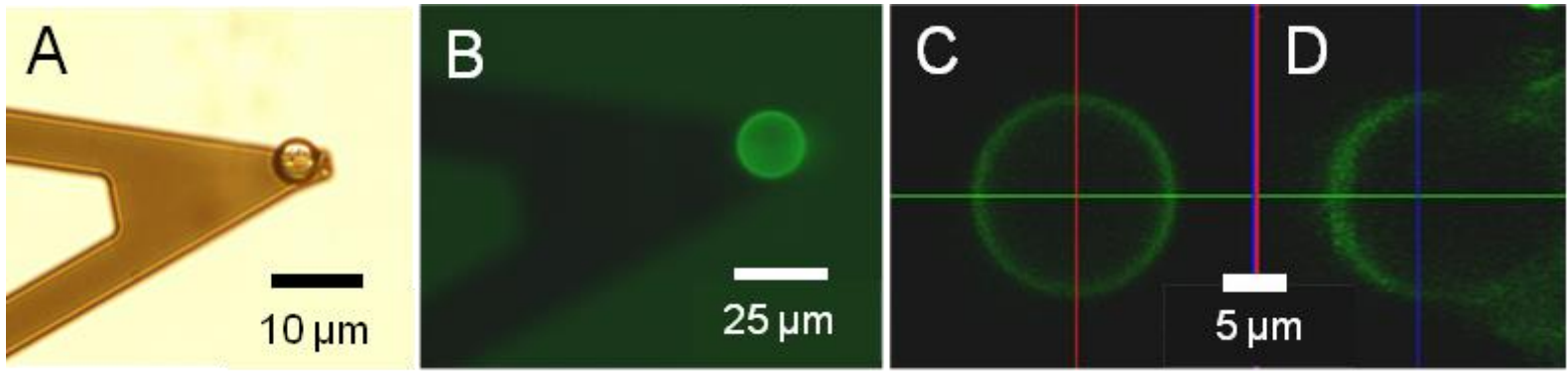

Figure 5.3: Membrane coating of a colloidal probe attached to MLCT-OW cantilever. A. Light microscopy image of the front end of a colloidal probe cantilever. ${ }^{[92], 71} \mathrm{~B}$. Epifluorescence image of the membrane coated colloidal probe cantilever (DOPC with BY) ${ }^{[92], 71}$ C, D: Confocal fluorescence images of the membrane-coated colloidal probe in the horizontal (C) and vertical (D) plane.

\footnotetext{
${ }^{71}$ Image reprinted from [92] with the permission of the American Chemical Society.
} 
Figure 5.3 shows a colloidal probe cantilever functionalized with a BY-labeled membrane. The light microscopic image (Figure 5.3-A) serves to control the correct positioning of the bead prior to the functionalization procedure. Incubation with fluorophor-labeled SUVs gave membrane-coated colloidal probe cantilever exhibiting considerable fluorescent properties prior and after force spectroscopy measurements (Figure 5.3-B, C). In order to control the unilamellarity of the solid-supported bilayer on the probe, we determined the size distribution of SUVs to range from 30 to $50 \mathrm{~nm}$, which is in accordance with literature values rendering MLVs rather improbable. ${ }^{72,[96]}$

\subsubsection{Fluidity of the probe membrane}

Bleaching of the POPC/BY membrane on the colloidal probe cantilever and detection of fluorescence intensity after the bleaching pulse gave an indication the fluorescence intensity in the ROI recovers being equivalent to a diffusion of fluorescent lipids in the membrane. Diffusion coefficients were determined by using the Axelrod fit procedure. ${ }^{[111]}$

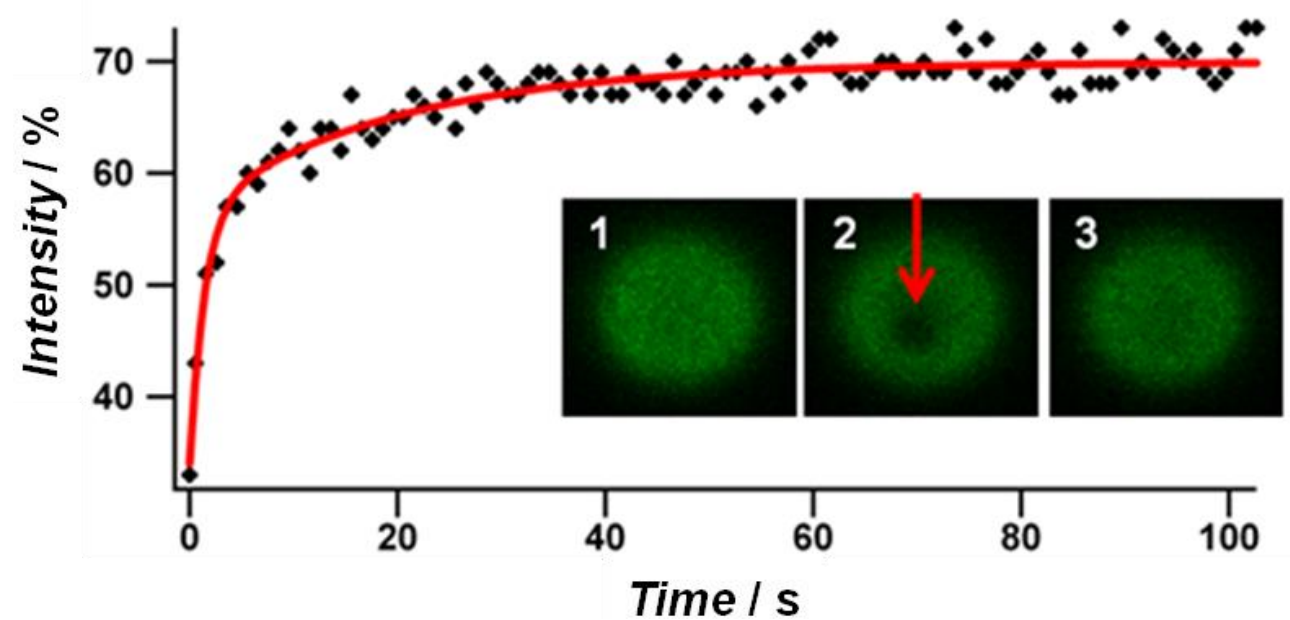

Figure 5.4: Fluorescence intensity recovery curve as detected after bleaching a DOPC/BY membrane probe. ${ }^{[75], 73}$ Intensity profile of the fluorescence recovery after photobleaching reconstructed from the corresponding fluorescence images (1: before bleaching, 2: 800 ms, and 3: 40 s after bleaching). The mean diffusion coefficient determined from fitting of the intensity recovery as a function of time was $1.4 \pm 0.1 \mathrm{\mu m}^{2} / \mathrm{s}$. Within 5 seconds, $60 \%$ of the original intensity has recovered. The red arrow indicates the bleached spot, which is the region of interest that has been analyzed to give the recovery curve.

\footnotetext{
${ }^{72}$ DLS measurements were performed by Gesa Pähler

${ }^{73}$ Image reprinted from [75] with the permission of Elsevier.
} 
In the case of DOPC/BY-coated probes (Figure 5.4, inset) diffusion coefficients were determined to be $1.4 \pm 0.1 \mu \mathrm{m}^{2} / \mathrm{s}$. Recovery of fluorescence in the bleached area was achieved after $100 \mathrm{~s}$. The recovery was incomplete due to an exhausting membrane reservoir on the sphere. When using aggregated

SUVs for the incubation of the colloidal probe, micrometer-sized fluorescent aggregates were observed on the cantilever. No fluorescence recovery was detectable after bleaching the material on the probe.

\subsubsection{Integrity of membranes on wafer}

Figure 5.5 shows supported lipid bilayers prior to a force spectroscopy measurement as detected by fluorescence (POPC membranes on silicon wafer, A) and by AFM imaging (DPPC membrane on silicon wafer, B). To quick-check the membrane formation process, POPC samples were manipulated with a soft pipette tip as depicted in Figure 5.5.
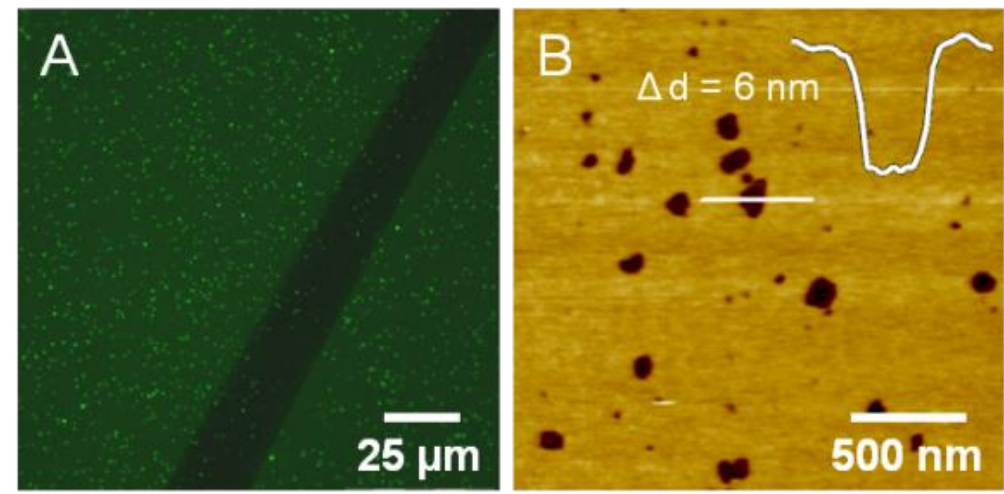

Figure 5.5: Solid-supported lipid bilayers as used for membrane probe experiments. A: Epifluorescence image of a BY-labeled POPC membrane. B: Intermittent contact mode image of DPPC bilayer exhibiting membrane defects with a height of $6 \mathrm{~nm}$.

Intensity differences were interpreted as the border between membrane-coated and uncoated areas. Darker spots in the image represent membrane defects displaying a depth of about $6 \mathrm{~nm}$. For force spectroscopy measurements only samples with a membrane surface coverage of more than $90 \%$ were accepted. 


\subsubsection{Discussion}

In order to allow for free diffusion of membranes on solid supports, a limited roughness of surfaces is crucial for membrane probe setups. Root mean square $(r m s)$ roughness values of borosilicate beads (Duke borosilicate glass 9020, Duke Scientific diameter: $20 \mu \mathrm{m}$ ) have been determined by Van Zwol through raster-scanning the colloidal probe on an array of sharp tips to be below $1 \mathrm{~nm}$ on a $(20 \times 20) \mu \mathrm{m}^{2}$ area. ${ }^{[134]}$ Their results are in good accordance with our results obtained from imaging a colloidal probe cantilever surface with a conventional cantilever in contact mode $(r m s \sim 0.3$ to $0.7 \mathrm{~nm}) .{ }^{74}$ The calculated roughness value is minor (5\%) compared to the membrane thickness, which excludes a possible influence of local surface curvature on membrane integrity.

Successful formation of membranes on solid supports was visualized by fluorescence microscopy techniques and AFM imaging. Lateral mobility of components in fluid phase membranes was proven by the determination of diffusion coefficients by FRAP measurements, which were determined to range from 1.4 to $6 \mu \mathrm{m}^{2} / \mathrm{s}$ being in good agreement with values extracted from measurements on giant vesicles and free-standing bilayers. ${ }^{[16]}$
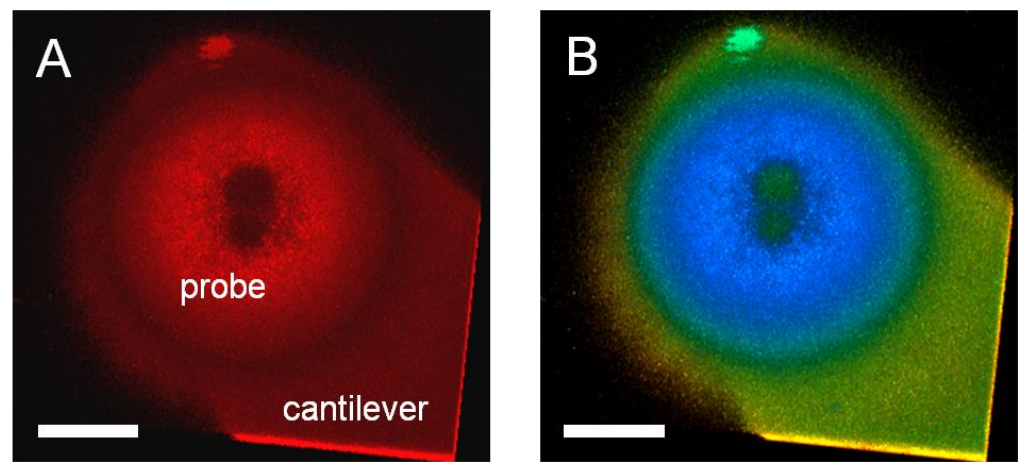

Figure 5.6: Top view on a three-dimensional CLSM image of a TR-labeled DPPC membrane probe, which has been produced by incubating the colloidal probe cantilever with gel phase vesicles at $55{ }^{\circ} \mathrm{C}$ for 30 min. ${ }^{75}$ For incubation the colloidal probe cantilever was fixed in a PTFE chamber suited for CLSM measurements and coated with a SUV suspension. After rinsing the lever, imaging was carried out with a $63 \mathrm{x}$ magnification at an excitation wavelength of $561 \mathrm{~nm}$. The homogeneous fluorescence on the bead surface is characteristic for a fluorescently labeled membrane on the probe. Dark areas (A) or green areas (B) at the center of the probe show two different sized spots, which have been bleached with a high intensity laser before performing the $Z$ stack being indicative for the immobility of the gel phase membrane.

\footnotetext{
${ }^{74} \mathrm{rms}$ value determined by using the roughness tool of SPIP program (parameter sq)

${ }^{75}$ Brightness and contrast of images has been increased for presentation purposes.
} 
However, it has to be noted that the Axelrod tool is not perfectly suited for our system as it assumes diffusion on planar surfaces and resulted in variances in diffusion coefficients of up to $50 \%$ upon repeated analysis of the same data sets. Hence, we state that membranes on colloidal probes are laterally mobile but we refrain from a quantitative analysis.

Besides fabricating fluid phase membrane probes, we also coated colloidal probes with gel phase membranes (Figure 5.6), which is feasible but involves the risk of damaging the cantilever upon heating the sample above the transition temperature of the lipid and of exposing the membrane to the air-water interface upon transferring the cantilever from the heating chamber to the AFM setup.

For the analysis of membrane fusion processes it is important to know if single lipid bilayers or bilayer stacks are being measured. Therefore, we determined vesicle size distributions in order to get an estimate of unilamellarity of membranes. From a narrow vesicle size distribution and opalescent vesicle suspensions, we conclude that we are dealing with unilamellar vesicles. Furthermore, force-distance curves with freshly prepared membrane probes taken on sufficiently hard surfaces show only single breakthrough events at high force loads (8 $\mathrm{nN}$ force load; not shown) suggesting unilamellarity of the adsorbed bilayers on the spheres. ${ }^{[135]}$. Moreover, we consider spreading of vesicles on top of present membranes unlikely assuming that we deal with unilamellar membranes.

Throughout the force spectroscopy measurements, background forces determined from probing control samples lacking membrane functionalization to be around $30 \mathrm{pN}$, which is attributed to non-specific membrane interaction. We conclude, that the fluidity of the bilayer paired with an abundance of choline headgroups disfavors non-specific interactions to a large extent. 


\subsection{Technical aspects of membrane probe spectroscopy measurements}

Equilibration times of five minutes prior to force spectroscopy measurements turned out to be sufficient to largely exclude drift of the colloidal probe cantilever deflection. However, some drift in the deflection $D$ has been observed after several force curves or force maps and was corrected by readjusting the position of the reflected beam on the photodiode to the center of the diode. Spring constant determination yielded normal deviations of manufacturer's values. Loss of the sphere occurs in less than $5 \%$ of the spectroscopy experiments if probes are properly attached as described in chapter 5.1.1. Due to drift effects, baseline tilting occurred leading to an undesirable change in contact force due to tilting of the whole curve.

To circumvent this, the application of a real time-baseline fitting procedure turned out to be useful. ${ }^{76}$ Due to hydrodynamic drag effects on the colloidal probe cantilever velocities higher than $10 \mu \mathrm{m} / \mathrm{s}$ were avoided.

If high loading forces were required, colloidal probe cantilevers with higher $k_{c}$ values were employed. Interestingly, we observed an effect of the approach velocity on the maximum adhesion force detected upon retraction of membrane probe cantilevers. Significantly larger adhesion forces were measured when the probe was approached to the surface at a low velocity. This could be due to hydrodynamic effects, which affects the binding capacity of molecules on the surface or due to an increased receptor-ligand contact time allowing for a notably higher binding probability. We also observed that the integrity of the membrane on the colloidal probe cantilever can suffer from a high number of performed force cycles, especially while probing strong interactions.

${ }^{76}$ vertical line deflection option in MFP3D tools

A Force Spectroscopy Setup to Mimic Cellular Interaction Processes 


\subsection{The strength of the $\mathrm{Ni}^{2+}-\mathrm{NTA}-\mathrm{H} 6$ interaction system}

\subsubsection{The membrane probe setup for probing the $\mathrm{Ni}^{2+}-\mathrm{NTA}-\mathrm{H} 6$ interaction}

Fluid-phase DOPC bilayer were chosen for functionalization of the colloidal probe to ensure sufficient lateral mobility of the lipids and as a consequence to establish a satisfactory large number of recognition events during the membrane-membrane contact. Moreover, membranes in the fluid phase are more prone to fusion events due to substantially smaller bending moduli and area compressibility values.

\subsubsection{Functionalization of membranes with $\mathrm{Ni}^{2+}-\mathrm{NTA}$ and $\mathrm{H} 6$}

Quartz crystal microbalance measurements reported the successful immobilization of $\mathrm{H} 6$ peptides on a DOPC/MCC-DOPE (90:10) membrane and the subsequent binding of $\mathrm{Ni}^{2+}$ NTA-decorated vesicles. ${ }^{77}$

Addition of functionalized vesicles resulted in a large frequency drop associated with an increase in dissipation. These changes are ascribed to the increase of viscoelastic mass on the oscillation quartz, which is assigned to the coordination binding of vesicles to the histidine moieties of $\mathrm{H} 6$ peptides on the membrane. More information about QCM-D measurements is given in chapter 8.3.1.

\subsubsection{Adhesion force and work of adhesion of the $\mathrm{Ni}^{2+}-\mathrm{NTA}-\mathrm{H} 6$ interaction}

A typical force-distance curve obtained from probing the interaction strength between $\mathrm{Ni}^{2+}$ binding sites on the colloidal membrane and histidine residues on the membrane supported by a silicon wafer. It is characterized by an initial adhesion regime equal to a large deflection of the cantilever towards the surface. While the breakage of initial interactions is characterized by a saw-tooth-like pattern the extension of tethers far away from the surface is distinguished by force plateaus.

\footnotetext{
${ }^{77}$ Measurements were performed by Rabea Keller during her diploma thesis in the group 


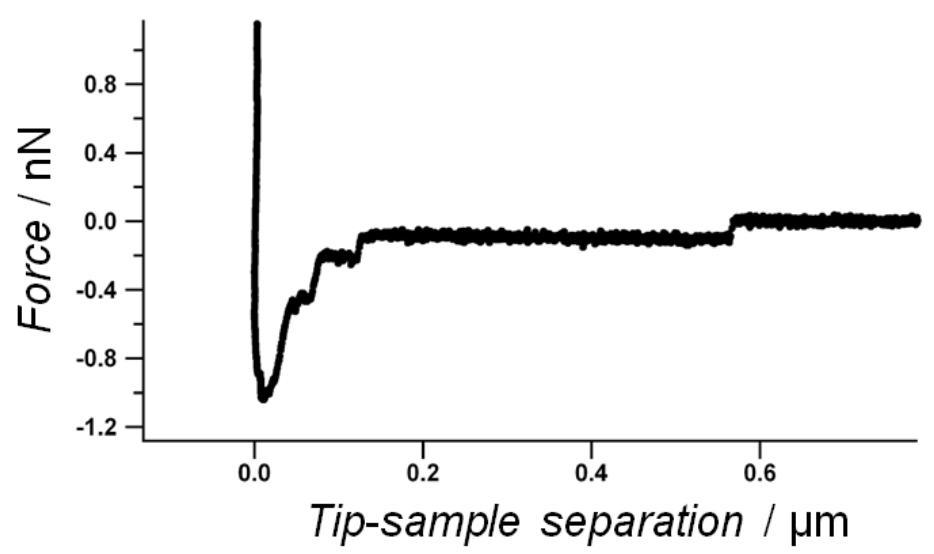

Figure 5.7: Typical force retraction curve obtained after contacting a membrane (DOPC/DOGS-NTA-Ni ${ }^{2+}$, 90:10) covered colloidal probe with a planar bilayer equipped with His-tag peptides (DPPC/MCC-DPPE$\left.\mathrm{H}_{6} \mathrm{GGC}, 90: 10\right) .{ }^{[75], 78}$ As depicted in Figure 3.16, we extracted the work of adhesion $W_{a d}$, the maximum adhesion force $\boldsymbol{F}_{a d}$, the number of tethers, the tether length $\boldsymbol{l}_{\text {tether, }}$ lifetime $\tau$ and tether rupture forces $\boldsymbol{F}_{\text {rup }}$. The force curve has been recorded with a pulling speed of $2.5 \mu \mathrm{m} / \mathrm{s}$ and a dwell time on the surface of $1 \mathrm{~s}$.

The constant force observed in tether elongation is solely determined by intrinsic viscoelastic parameters associated with the bilayer and the retraction velocity. ${ }^{[56,58]}$ In the case of multiple tether formation, the subsequent rupture of tethers produces a staircase-like retraction curve showing several plateaus of constant force. ${ }^{[57]}$ These plateaus eventually disappear in a single relaxation step indicating tether rupture. In a distance of $600 \mathrm{~nm}$ to the surface no force is acting on the cantilever and it snaps back to zero deflection. Appropriate control experiments were carried out to exclude that non-specific interactions interfere with the force response. The most straightforward way to extract quantitative data from force retraction curves is to register the maximum adhesion force $F_{a d}$ and the work of adhesion $W_{a d}$ which is essentially the integral as denoted in Figure 3.16, and cast the individual values into a histogram.

Hence, we monitored the maximum adhesion force and the surface adhesion energy in the absence of the ligand $\mathrm{H} 6$ coupled to the planar membrane. We found a mean maximum adhesion force of $(80 \pm 190) \mathrm{pN}$ for control experiments lacking the ligand H6 (Figure 5.8-A), while a more than 10 times higher adhesion force was found in presence of phospholipids displaying the ligand $\mathrm{H} 6(880 \pm 940) \mathrm{pN}$ (Figure 5.8-B). The overall forces in this case ranged from $100 \mathrm{pN}$ to $3 \mathrm{nN}$.

\footnotetext{
${ }^{78}$ Figure adapted from reference [75]. 
The difference is even more pronounced for the adhesion energies, which is probably due to the augmented formation of membrane tethers once $\mathrm{Ni}^{2+}-\mathrm{NTA}$ bearing lipids form complexes with phospholipids displaying $\mathrm{H} 6$ tags. Notably, we use the term work of adhesion being distinct from the free energy of adhesion since the experiment is carried out under nonequilibrium conditions. Control experiments in the absence of $\mathrm{H} 6$ produce a work of adhesion of merely $(2.7 \pm 3.4)$ aJ in contrast to $(97 \pm 137)$ aJ for experiments carried out in the presence of lipids functionalized with $\mathrm{H} 6$.

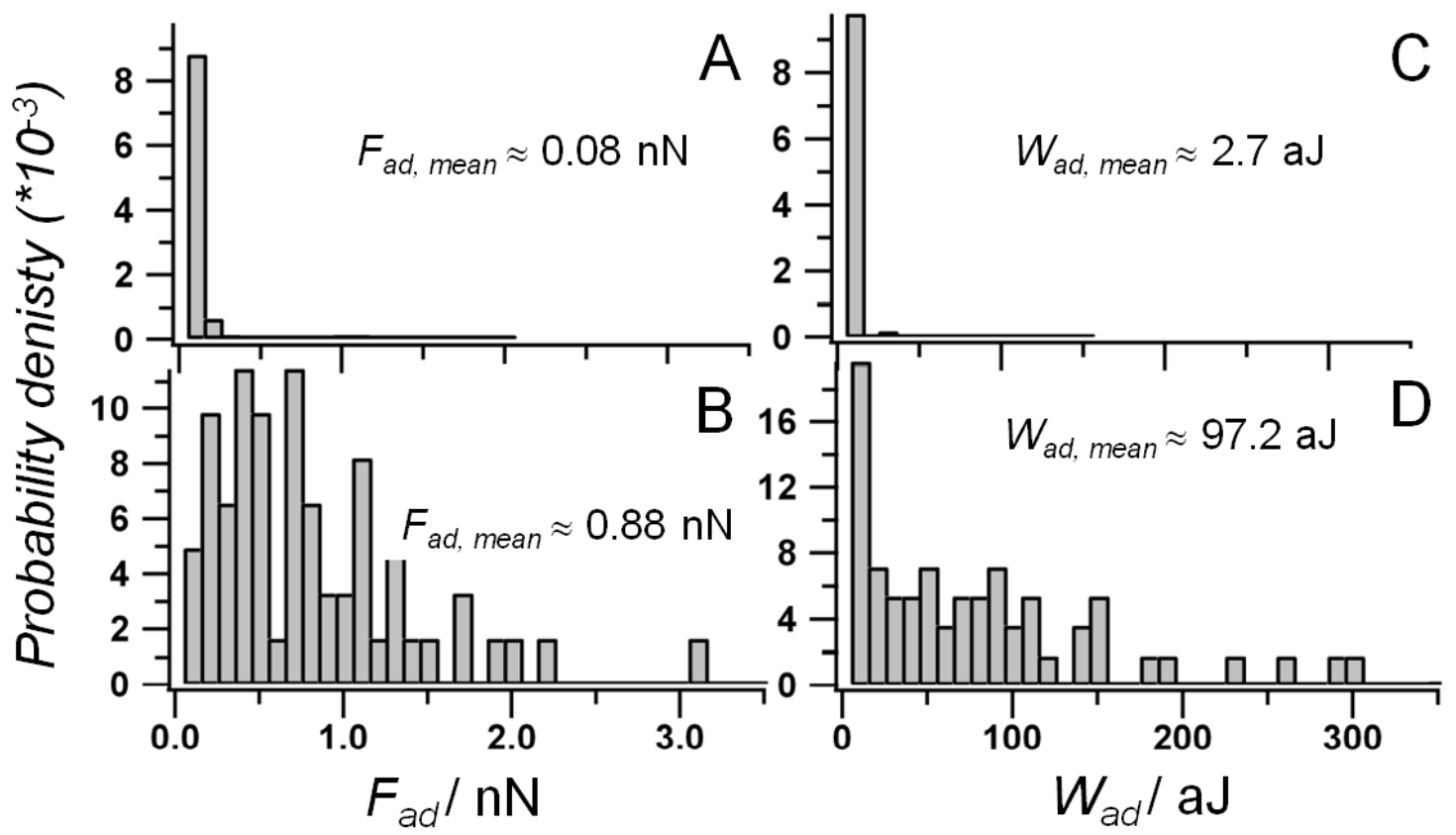

Figure 5.8: Histograms of maximum adhesion forces $F_{a d}(A, B)$ and work of adhesion $W_{a d}(C, D)$ obtained from integration of the retraction force curve from the point of contact to the end of piezo travel as depicted in figure 5.7. ${ }^{[75], 79}$ Control experiments in the absence of $\mathrm{H} 6$ tags $(A, C)$ show at least 10 times smaller values than membrane probe experiments allowing for the formation of ligand-receptor bonds between the two membranes (B, D). Force curves have been recorded with a pulling speed of $2.5 \mu \mathrm{m} / \mathrm{s}$ and a dwell time on the surface of $1 \mathrm{~s}$. Data was pooled from 60 force curves with two different cantilevers for His-tag experiments and from 60 force curves with three different cantilevers for control experiments.

The experiments clearly reveal that strong adhesion forces/energies result only if noncovalent linkages are allowed to form upon contact of the two membranes. It is, however, unclear whether unbinding of $\mathrm{Ni}^{2+}$-NTA-His-tag complexes is detected or membranes join at least partly thus forming a continuous leaflet, which eventually ruptures upon extension.

\footnotetext{
${ }^{79}$ Image reprinted from [75] with the permission of Elsevier.
} 


\subsubsection{Determination of formed ligand-receptor bonds}

It is instructive to first determine how many bonds may have been formed upon full contact of the two approaching membranes. The defined geometry of the colloidal probe permits a semi quantitative analysis. After estimation of the approximate contact area between colloidal probe and the bilayer covered silicon wafer the information was inserted into a Monte Carlo simulation in order to extract more details about the number of possible contacts. ${ }^{80}$ Following the theory of Hertz, being the most vigorous simplification of all contact models and neglecting the impact of adhesive forces on the contact mechanics, the contact area depends on the external load force $F_{\text {load, }}$ the radius of the colloidal probe $r_{C P \text {, }}$ and the Young's moduli $E$ of the corresponding materials. ${ }^{[136]}$

A further simplification arises by assuming that the indentation depth is below $10 \%$ of the bilayer thickness permitting us to consider the two membranes as semi-infinite bulk material with a Young's modulus of approximately $10 \mathrm{MPa}$ (Poisson ratio: $v=0.33$ ). ${ }^{[137]}$ The spheres used in our study have a radius of $7.5 \mu \mathrm{m}$ and the load forces is at minimum $200 \mathrm{pN}$ producing an overall indentation depth of $0.5 \mathrm{~nm}$. We calculate a contact radius of $\mathrm{a}=60 \mathrm{~nm}$ at $\mathrm{a}$ load force of $200 \mathrm{pN}$ corresponding to a contact area of $\sim 11,000 \mathrm{~nm}^{2}$. The number of lipid molecules within the contact area can be obtained from knowledge of the cross sectional area of a single lipid molecule $\left(A_{\text {lipid }}=0.7 \mathrm{~nm}^{2}\right)$.

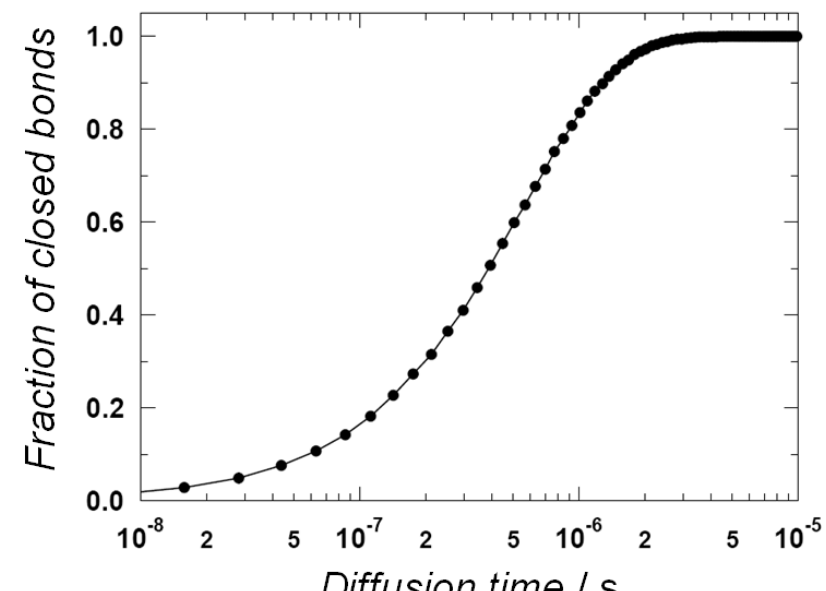

Figure 5.9: Monte Carlo simulation showing the fraction of bonds formed as a function of contact time. ${ }^{79,80}$ The graph shows the fraction of closed bonds versus diffusion time calculated for a two-dimensional random walk of molecules. The fraction of closed bonds reaches 1 already after diffusion times in the $\mu s-$ region.

${ }^{80}$ Monte Carlo simulations were performed by Eva Sunnick, University of Göttingen. 
Hence, a load force of merely 200 pN brings approximately 15,600 lipids in close contact. Since the membranes are doped with $10 \%$ of receptors or ligands, respectively, we can estimate that at most 1,600 molecular bonds can form allowing saturation of bonds.

In order to obtain a more realistic number of ligand-receptor-linkages, we performed a simple simulation based on two approaching surfaces with randomly placed ligands and receptors occupying an area of $10 \%$ (Figure 5.9). The number of irreversibly formed contacts is counted and lateral mobility is represented by a so-called affinity radius that increases with contact time according to the classical two-dimensional random walk of lipid molecules. Every pair at a distance smaller than the affinity radius is counted as a bond. Opening of bonds is not considered in order to simplify the procedure leading to the estimation of an upper limit of bonds. We found that diffusion of receptor and ligand molecules is fast enough to allow all possible configurations within one millisecond. After $1 \mathrm{~s}$ dwell time of the colloidal probe in contact with the opposing membrane we can safely assume that all possible bonds are formed at a load force of $200 \mathrm{pN}$.

\subsubsection{Wearing-off of the system}

After recording of more than 100 force-distance curves with the $\mathrm{Ni}^{2+}$-NTA-His-tag setup an ablation of membrane material from the probe has been observed (Figure 5.10-A/B). We ascribe this fact to the quasi-covalent bond formed between the contacting membranes leading to the fading of membrane material off the probe. This is in accordance with results obtained from the analysis of $F_{a d}$ and $W_{a d}$ values detected in consecutive force distance curves (Figure 5.10-C). 

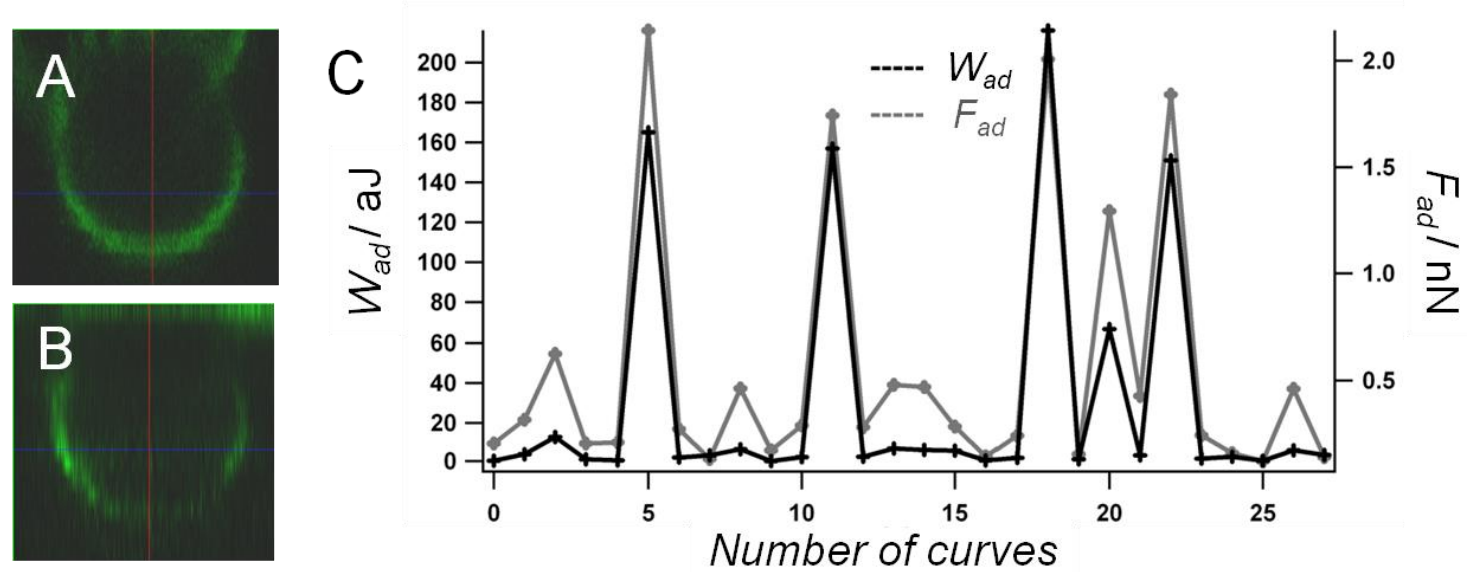

Figure 5.10: Ablation of the membrane material upon probing interactions. ${ }^{[75], 79} \mathrm{CLSM}$ images (cross section in the $x-z$ plane) of colloidal probes covered with DOPC/DOGS-NTA (89:10) bilayers labeled with BY (1 mol\%) before (A) and after (B) carrying force distance curves on bilayers doped with the corresponding ligand (H6). Note that membrane patches are missing after the experiment indicative of exhausted membrane reservoirs after tether pulling. C: Work of adhesion (black) and adhesion force (gray) of consecutive force distance curves performed with (DOPC/DOGS-NTA-Ni ${ }^{2+}, 90: 10$ ) bilayer coated colloidal probe in contact with (DPPC/MCC-DPPE-H 6 GGC; 90:10) bilayer. We attribute the frequent drops in adhesion to loss of membrane material after tether formation. Diffusion leads to a temporary membrane healing illustrated by higher adhesion values.

It is striking that values increase by a factor of 10 in a regular intervals. We interpret this as an additional indication for an ablation of membrane material due to the strong interaction between $\mathrm{Ni}^{2+}$-NTA moieties and His-tagged peptides.

\subsubsection{The approach curve - hemifusion instead of docking?}

Membrane probe experiments with DOPC/DOGS-NTA (90:10) coated spheres approaching either planar DPPC or DOPC bilayers equipped with the corresponding ligand $\mathrm{H} 6$ show similar trajectories on approach and retraction of the cantilever. In both cases bilayer instabilities are accompanied by formation of membrane tethers (Figure 5.11). The instabilities after contact are clearly visible and a sudden drop of the contact force occurs indicative of a mono- or bilayer breakthrough. DOPC membranes, however, are more prone to two subsequent breakthrough events implying full fusion of the two DOPC bilayers, while DPPC bilayers are naturally more stable exhibiting single instabilities. 


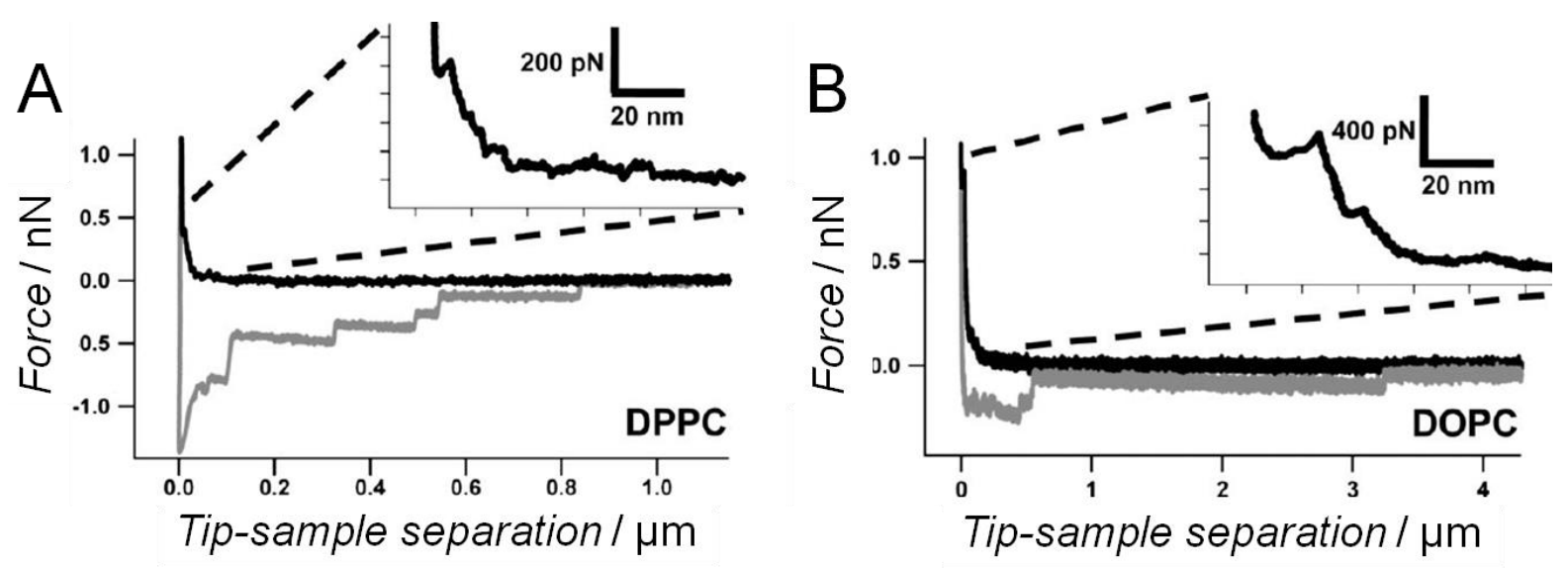

Figure 5.11: Analysis of force-distance approach curves in the presence of H6 equipped lipids. ${ }^{[75], 79}$ While the colloidal probe approaches the surface, instabilities are detected within a distance of $20 \mathrm{~nm}$ to the surface. A: Approach and retraction curves of a colloidal probe covered with DOPC/DOGS-NTA- Ni ${ }^{2+}$ (90:10) touching a DPPC/MCC-DPPE- $\mathrm{H}_{6}$ GGC (90:10) bilayer on planar silicon. A single instability is found in the approach curve (inset) followed by multiple tethers upon retraction of the cantilever. B: Approach and retraction curves of a colloidal probe covered with DOPC/ DOGS-NTA- $\mathrm{Ni}^{2+}(90: 10)$ touching a DOPC/MCC-DOPE- $\mathrm{H}_{6}$ GGC $(90: 10)$ bilayer. Two instabilities are found in the approach curve (inset) followed by a single tether upon retraction of the cantilever.

We consider these observations, which correlate with the occurrence of tethers in the retraction curve, as a first indication that at least hemifusion events take place.

\subsubsection{Stochastic bond breakage or membrane fusion}

Once the membranes have been brought into contact, two scenarios are conceivable upon retracting the colloidal probe from the substrate. The established bonds between the $\mathrm{Ni}^{2+}-$ NTA lipids and the corresponding His-tag carrying lipids break as the force acting on them increases and the measured adhesion force reflects the stochastic nature of parallel bond breakage. Alternatively, membranes merge upon contact and the attractive forces are due to formation of continuous leaflets covering the colloidal probe and the silicon substrate. Fusion between the two membranes might be only partial and restricted to the outer leaflet, which is referred to as hemifusion. Full fusion requires both leaflets to join thus establishing a continuous bilayer. The task is now to dissect the different scenarios by careful analysis of the force distance curves. One way would be to extrapolate the measured adhesion forces to the mean rupture force of a single bond and compare the results with the known potential of the $\mathrm{Ni}^{2+}$ NTA-His-tag complex from single molecule force experiments. ${ }^{[1]}$ Previous studies on the stochastic bond breakage of $\mathrm{Ni}^{2+}-\mathrm{NTA}-\mathrm{His}$-tag bonds with an atomic force microscope pro- 
vide us with the kinetic key parameters $k_{\text {off }}(0), \Delta G^{\circ}(F)$, and $x_{u}$ as well as rupture forces $F_{\text {rup }}$ at specific loading rate $r_{f}$. Following a protocol established by Beebe and coworkers we extrapolated our $F_{a d}$ data to the failure of a single bond under external load assuming that the variance of adhesion forces obtained in the different experiments is only caused by a variation in the number of formed bonds. ${ }^{[138]}$ Assuming further that the rupture events obey a Poisson distribution, one can plot the average force as a function of the variance and extract the dynamic bond strength of an individual bond from the slope. Trying this, we could not find the predicted linear relationship between the average force and its variance. As a consequence, the procedure of Beebe et al. is not purposeful in our case, either because the distribution of forces does not follow a Poisson distribution or because the governing connection between probe and sample is different from stochastic ligand-receptor bonds.

\subsubsection{Lipid tethers are formed between the membranes}

A second way to find out whether membranes have merged upon contact relies on registering of tether lifetimes. Tethers are characterized by constant force $F$ upon extraction, which depends only on intrinsic mechanical parameters of the bilayer such as the bending modulus $\kappa$, the tension $\sigma$, and the shear viscosity $\eta$.

$$
F=2 \pi \sqrt{2 \kappa \sigma}-2 \pi \eta v
$$

\section{Equation 9}

Due to the viscous contribution the force depends also on the pulling speed. Equation 8 can be used to extract intrinsic mechanical parameters from the tether pulling experiments. A plot of the tether force as a function of pulling velocity can be used to determine the apparent tension $\sigma$ that also represents the adhesion of the bilayer to the colloidal sphere. We assume a bending modulus $\mathrm{k}$ of $10^{-19} \mathrm{~J}$ corresponding to a fluid bilayer and thus find an apparent tension of $1.2 \mathrm{mN} / \mathrm{m}$. The value corresponds well to recent findings from indentation of pore spanning bilayer and is attributed to large lateral tension $\sigma$ due to strong adhesion to the surface ${ }^{[139]}$ Once the tether is formed, its radius is constant and does only depend on the viscoelastic properties of the membrane. ${ }^{[56]}$

The tether radius $r_{\text {tether }}$ can be readily obtained from Equation 10:[140] 


$$
r_{\text {tether }}=\frac{2 \pi \kappa}{F_{\text {tether }}}
$$

Equation 10

Assuming an equilibrium tether force $F_{\text {tether }}$ of $80 \mathrm{pN}$, a tether radius of $6-10 \mathrm{~nm}$ is found. The fact that tethers are formed upon pulling the probe away from the surface alone does not elucidate, which bonds are strained and eventually failure. It just tells us that the membrane or at least one leaflet is lifted off from the support overcoming the adhesion force between the bilayer and the substrate. However, since the tether length is a measure of the bond's lifetime it can be used to estimate the off-rates at constant force $k_{\text {off }}(0)$ and compare the values to the known off-rates of the $\mathrm{Ni}^{2+}-\mathrm{NTA}-\mathrm{His}$ tag bonds. ${ }^{[71]}$ In many tether pulling experiments, it is assumed that the molecular bond attaching the probe or pulling device to the membrane remains intact. The lifetime of the bond is, as described in 4, a function of external force and the energy landscape, which is usually dominated by a single prominent energy barrier continuously lowered by the applied force ramp. Tethers can be used to create a constant force only depending on the mechanical properties of the membrane and the pulling velocity. Müller et al. introduced a method to measure the lifetime of single bonds at a defined constant force using membrane tethers pulled from cells at varying velocities. ${ }^{[58]}$ We adopted this procedure to judge whether stochastic bond rupture governs the process or hemifusion took place prior to retraction. In membrane probe experiments with $\mathrm{Ni}^{2+}-\mathrm{NTA}-\mathrm{H} 6$, usually multiple tethers were formed and a staircase force profile was observed (Figure 5.7 and Figure 5.11-A). Analysis of the tether frequency (how many tethers are observed in a set of force-distance curves) shows that in the control experiments lacking $\mathrm{H} 6$ with a contact time of one second the maximum number of tethers in one retraction curve is only one (Figure $5.12-\mathrm{A}$ ). About $85 \%$ of all the retraction curves showed no tether formation. In contrast, bilayers displaying $\mathrm{H} 6$ ligands showed a substantially higher tendency to form tethers. The highest number of tether plateaus observed in one retraction curve is eleven. We also observed force curves in which no tethers were formed. This, however, correlates with a loss of membrane material from the probe due to successful tether formation prior to the actual pulling experiment as described in chapter 5.3.5, Figure 5.10. 

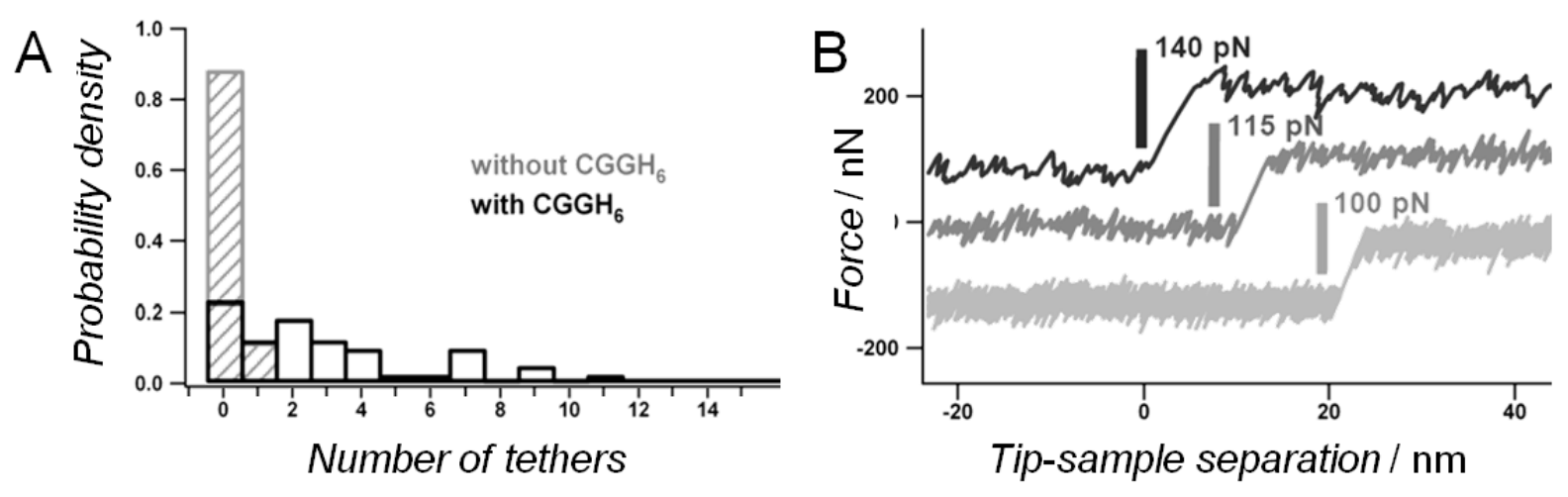

Figure 5.12: Frequency of tether formation found in retraction curves with and without H6. ${ }^{[75], 79}$ The number of extracted nanotubes during one retraction process increases up to $\boldsymbol{N}_{\text {tether }}=\mathbf{1 1}$ upon addition of $\mathrm{H6}$. B, C: Typical force retraction curves showing tethers with final rupture events at different pulling velocities (dark grey: $2.5 \mu \mathrm{m} / \mathrm{s}$, mid: $1 \mu \mathrm{m} / \mathrm{s}$, light grey: $0.1 \mu \mathrm{m} / \mathrm{s}$ ). Increased pulling velocities lead to increased tether rupture forces as expected from Equation 9.

This means that consecutive force curves are frequently devoid of tether events due to an exhausted membrane reservoir on the spheres. The mean number of tethers per retraction curve for control experiments is $N_{\text {tether }}=0.12$, whereas it is $N_{\text {tether }}=3.6$ for experiments with $\mathrm{H} 6$ ligands. The number of tethers increases with the dwell time of the probe in contact with the surface.

In the following, we describe a protocol developed by Müller and coworkers to obtain off rates from force clamp conditions. Hence, we monitored the lifetime of the tethers - as computed from the tether length by dividing by the pulling velocity - as a function of tether force (Figure 5.12-B). Figure 5.13 shows the resulting histograms of tether lifetimes $\tau$ as a function of tether force adjusted by the pulling velocity. As expected, the lifetime increases with decreasing pulling speed. Assuming stochastic unbinding of a ligand-receptor pair, the unbinding probability $\mathrm{S}(\mathrm{t})$ is described by a single exponential function:

$$
S(t)=\exp \left(\frac{-t}{\tau}\right)
$$

Equation 11

The corresponding probability density is fitted to the normalized histograms providing a mean lifetime of $(1.27 \pm 0.39) \mathrm{s}$ for a pulling speed of $0.1 \mu \mathrm{m} / \mathrm{s},(0.21 \pm 0.02) \mathrm{s}$ for $1 \mu \mathrm{m} / \mathrm{s}$, and $(0.11 \pm 0.01)$ seconds for a velocity of $2.5 \mu \mathrm{m} / \mathrm{s}$. 
We also determined the lifetime of tethers $\tau(F)$ at a particular force (last tethers).

$$
k_{\text {off }}(F)=\tau(F)^{-1}
$$

Equation 12

We arrived at $(3.6 \pm 0.4) \mathrm{s}$ for a pulling speed of $0.1 \mu \mathrm{m} / \mathrm{s}$, $(0.32 \pm 0.05) \mathrm{s}$ for $1 \mu \mathrm{m} / \mathrm{s}$, and $(0.18 \pm 0.02) \mathrm{s}$ for $2.5 \mu \mathrm{m} / \mathrm{s}$. Employing the Bell model that describes the obtained rates as a function of force allows us to determine both $x_{u}$ the potential width, and $k_{\text {off }}$ at zero force $\left(k_{\text {off }}(0)\right) .{ }^{[131]}$ In the applied range of forces, a $k_{\text {off }}(0)$ was found to be $(0.63 \pm 0.44) \mathrm{s}^{-1}$ and $x_{u}=(0.28 \pm 0.04) \mathrm{nm}$. Notably, we considered only the last and therefore single tethers in order to avoid undefined forces acting on each individual tether. Only the last tethers are subject to a defined force (plateau force).
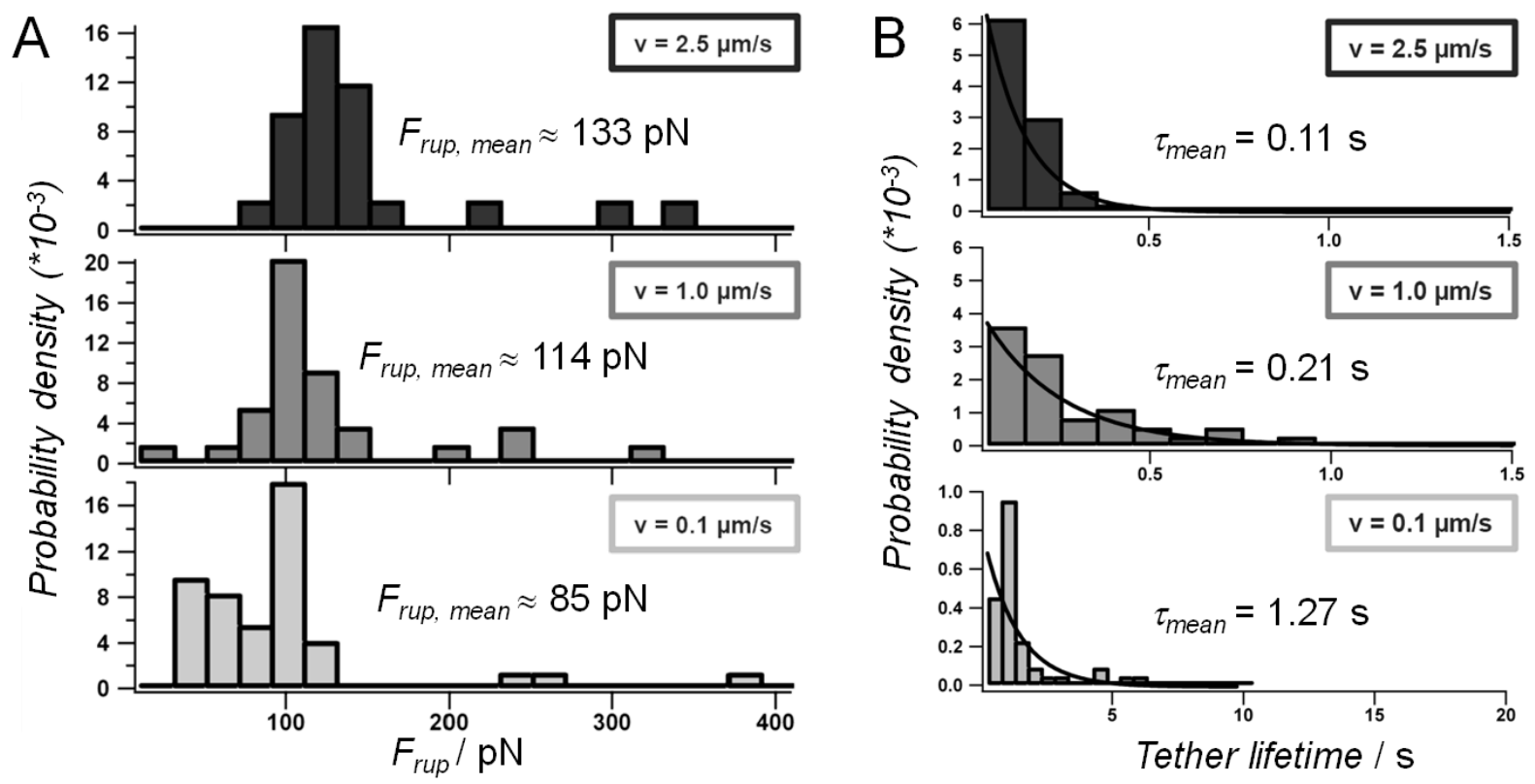

Figure 5.13: Tether rupture forces $f_{\text {rup }}$ and tether lifetimes $\tau$ at different pulling speeds. ${ }^{79}$ A: The rupture force histograms corresponding to the tether ruptures depicted in Figure 5.12-B exhibit mean rupture forces of $(133 \pm 65) \mathrm{pN}$ at $2.5 \mu \mathrm{m} / \mathrm{s},(114 \pm 59) \mathrm{pN}$ at $1 \mu \mathrm{m} / \mathrm{s}$, and $(85 \pm 68) \mathrm{pN}$ at $0.1 \mu \mathrm{m} / \mathrm{s}$. The histograms were calculated from measurements with two different cantilevers and 30 force curves for each velocity. B: The tether lifetime $\tau(F)$ increases with decreasing pulling speed ( $2.5 \mu \mathrm{m} / \mathrm{s}$ (top), $1 \mu \mathrm{m} / \mathrm{s}$ (middle), $0.1 \mu \mathrm{m} / \mathrm{s}$ (bottom)). ${ }^{[75]}$ Fitting of a monoexponential function results in mean lifetimes $\tau$ of $(0.11 \pm 0.01) \mathrm{s}$ at 2.5 $\mu \mathrm{m} / \mathrm{s},(0.21 \pm 0.02) \mathrm{s}$ at $1 \mu \mathrm{m} / \mathrm{s}$, and $(1.27 \pm 0.39) \mathrm{s}$ at $0.1 \mu \mathrm{m} / \mathrm{s}$ pulling speed. Lifetimes are obtained from division of the tether length by the pulling velocity. Histograms were calculated from measurements with two different cantilevers and $\mathbf{4 0}$ force curves for each pulling velocity.

Although the lifetimes are thus slightly overestimated we can use them as an upper bound in the following argumentation. Comparing the off-rates at zero load force $k_{\text {off }}(0)$ and the dis- 
tance from the ground state to the activation barrier $x_{u}$ to available literature values $\left(k_{\text {off }}(0)=0.07 \mathrm{~s}^{-1}, x_{u}=0.16 \mathrm{~nm}\right)$ for the same ligand receptor couple it is evident that there is not even a remote resemblance between our data and literature values. ${ }^{[71]}$

Particularly, the 10 times higher lifetime of $\mathrm{Ni}^{2+}-\mathrm{NTA}-\mathrm{His}$-tag bonds compared to our findings indicates that the anchorage of the tether is not established by one or more stochastic ligand-receptor bonds. In summary, we conclude that the linkage between the tether and the probe is most likely different from $\mathrm{Ni}^{2+}$-NTA chelating the corresponding His-tag $(\mathrm{H} 6, \mathrm{Ac}-$ $\mathrm{H}_{6} \mathrm{GGC}-\mathrm{NH}_{2}$ ). We rather think that hemifusion took place due to the contact pressure exerted by the AFM facilitated by formation of strong ligand receptor bonds merging the membranes at least partly. This assumption is supported by the observation that tether formation is related to single breakthrough events proceeding upon forced contact of the two membranes. As shown by Abdulreda et al. these small force jumps can be interpreted as hemifusion events. ${ }^{[66]}$

According to analysis of approach curves hemifusion and fusion are abundantly observed for two DOPC covered surfaces in contact. Even in the absence of ligand receptor pairs the two membranes merge if the membrane contact is induced by applying a critical joining pressure, which equals the undercutting of a certain distance. In contrast, predominantly hemifusion is found for DOPC/DPPC systems but only if defined molecular bonds are formed upon approaching the colloidal sphere. Scrutiny of colloidal probes covered with functionalized DOPC bilayers after performing force experiments on corresponding DPPC membranes clearly shows missing patches of membrane on the colloidal probe.

We conclude that rupture of tethers occurs due to stretching and failure of the DOPC bilayer while exhausting the reservoir on the silicon dioxide sphere. We assume that the DPPC bilayer on the flat silicon substrate remains largely unaffected upon retraction of the colloidal probe. Most likely, the fluid DOPC bilayer is peeled off the probe upon retraction forming a membrane tether, which is anchored in a hemifused fashion on the DPPC bilayer (Figure 5.14). Obviously, the formation of ligand receptor bonds is necessary to overcome the barrier for hemifusion of DOPC with the gel-phase bilayer. Moreover, we found that roughly the same tether length was extracted regardless of the pulling velocity, which we attribute to the finite lipid reservoir on the colloidal probe. 


\subsubsection{Model perception}

A number of different conceivable scenarios how (hemi)fusion takes place between the membrane coated colloidal probe and the planar solid supported membrane triggered by external force and formation of ligand receptor pairs exists. Figure 5.14 shows a collection of schemes that illustrate possible structures of the formed tethers.

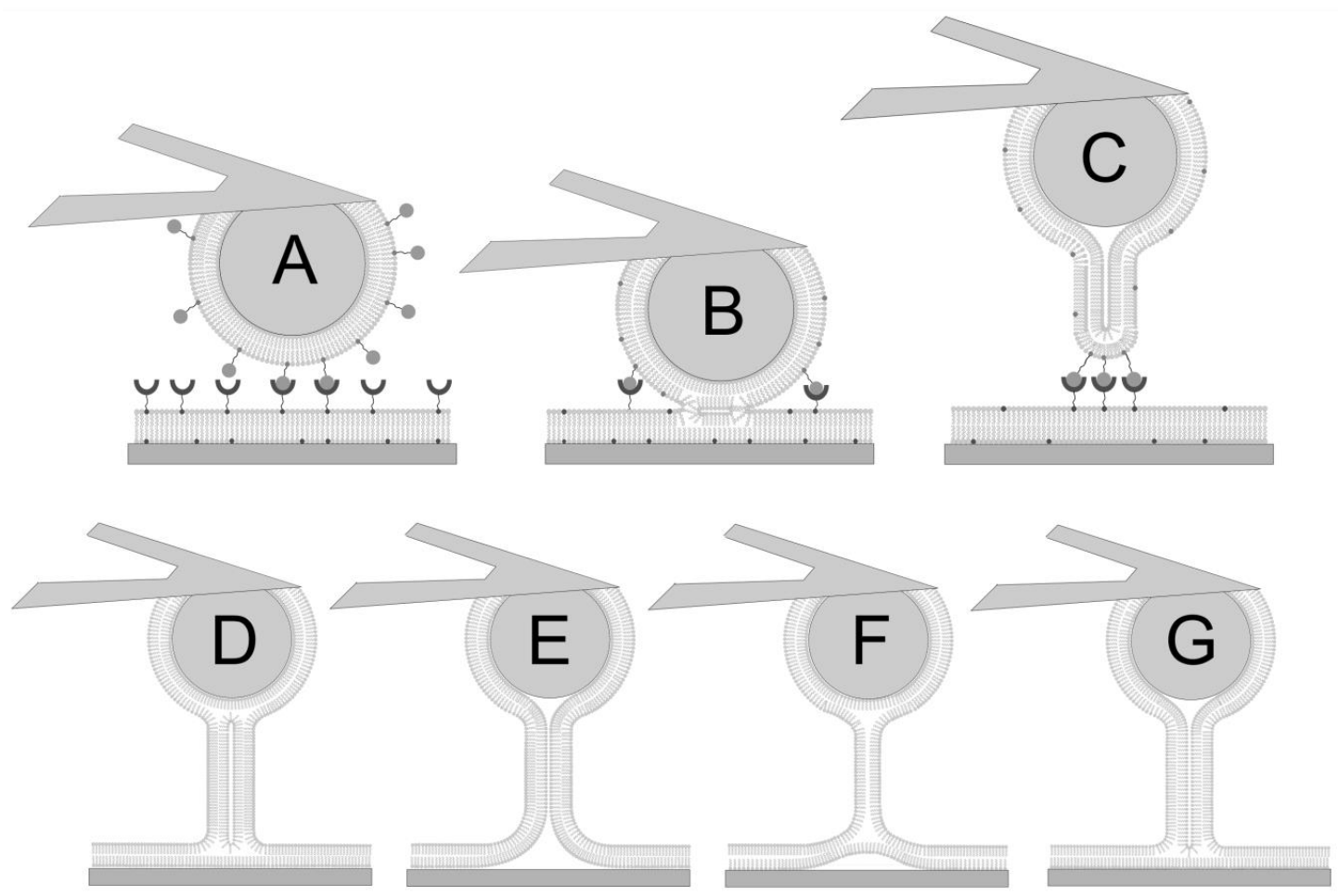

Figure 5.14: Probable scenarios for membrane-membrane interactions upon retraction of $\mathrm{Ni}^{2+}-\mathrm{NTA}^{-}$ functionalized probe from $\mathrm{H} 6$-functionalized substrate. ${ }^{[75], 79}$ Schematic illustrations showing possible structures of the formed tethers upon retraction of the colloidal probe from the bilayer covered substrate (A). Initial contact results in partial fusion of the two bilayers (B). Retraction of the colloidal probe results in tether formation $(C-G)$. Note that hemifusion of the outer leaflets of the two membranes (D, F, G) or full fusion (E) are conceivable.

Some of the scenarios can safely be excluded as deduced from the experimental evidence. The initial contact $(A)$ leads to partial merging of the two bilayers $(B)$ or to direct formation of a tether upon pulling $(C)$. Retraction of the colloidal probe $(C-G)$ might result in hemifusion of the outer leaflets of the two membranes $(D, F$, and $G)$ or alternatively to full fusion (E). $A$ subtlety occurs on the colloidal probe. The DOPC leaflet pointing to the sphere might detach ( $E$ and $G$ ) or remain in contact ( $D$ and $F$ ). Due to curvature penalty, structure $(F)$ is not very likely to occur. 
The estimated tether radius of $6-10 \mathrm{~nm}$ favors structures $C$ and $G$ because less energy is paid for bending and hydrophobic chains are not exposed to the aqueous environment. Since DPPC most likely does not participate in the tether formation at room temperature and hence does not act as a membrane reservoir upon pulling, we assume that the membrane reservoir consists exclusively of DOPC covering the colloidal sphere. This would also explain the relatively short tethers observed in the force retraction curves. We found considerably longer tethers when two DOPC membranes were brought into contact (Figure 5.11). From the force distance curves alone we cannot exclude that multiple bonds between $\mathrm{Ni}^{2+}-\mathrm{NTA}$ lipids and the corresponding His-tag form and sustain the pulling force (Figure 5.14-C). The weakest link is the membrane tube itself. However, the additional bulk experiment (dequenching assay) and QCM-D experiments reveal that hemifusion (lipid mixing) has taken place in DOPC experiments (chapter 8.3.1) ${ }^{81}$.

Taking all experimental evidence together, we can assume that Figure 5.14-G displays the most likely structure of the tether. In summary, we assume that the DOPC bilayer on the sphere feeds the tether, which is anchored in a hemifused manner onto the planar solid supported membrane. Whether full fusion or merely hemifusion occurs depends also on the externally applied force and the fluidity of the bilayers. While coating both, the colloidal probe and the flat substrate with DOPC might favor full fusion, DPPC on either the colloidal probe or the flat substrate is likely to prevent the formation of fully merged membranes.

\footnotetext{
${ }^{81}$ The reader is referred to reference 75. Lorenz, B., et al. (2010). "Colloidal probe microscopy of membranemembrane interactions: From ligand-receptor recognition to fusion events." Biophysical Chemistry 150(1-3): 5463.
} 


\subsection{Self-recognition between disaccharides derived from Microciona pro- lifera}

\subsubsection{Probing the homomeric Microciona prolifera interaction with a membrane probe setup}

A high surface density (10 mol\%) of disaccharides was chosen on both membranes to mimic the high density of sugars displayed by g-200 proteoglycans (Figure 5.15). DPPC was used to provide stability in the contact zone and to minimize detachment of the bilayer from the surface.
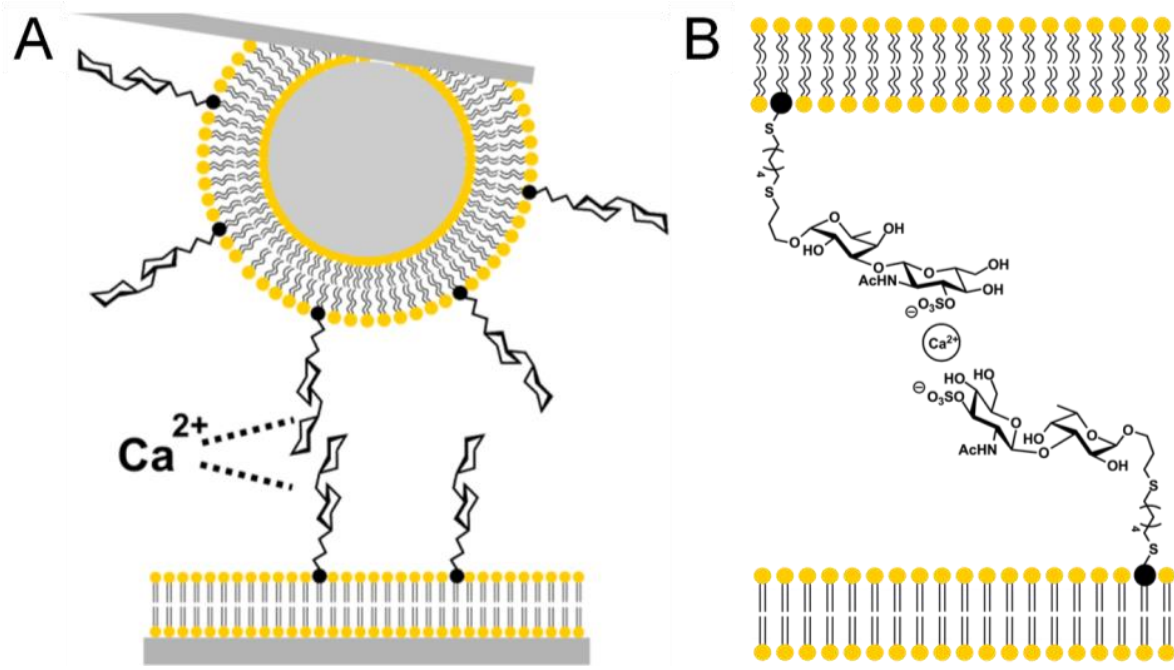

Figure 5.15: Colloidal force microscopy setup employing Microciona prolifera disaccharide structures. ${ }^{\text {[2],82 }}$ The $\mathrm{Ca}^{2+}$-dependent interaction is probed between membrane-coated surfaces $(\mathrm{A})$. The disaccharides are covalently coupled to the lipid bilayer through maleimide chemistry allowing for the probing of disaccharide interaction forces as a function of calcium ion concentration (B).

To allow for a rearrangement of membrane-bound disaccharide structures during the measurement, POPC was chosen to be the lipid matrix on the colloidal probe. To avoid that residual calcium ions influence the measurement, HEPES buffers for the negative control were supplied with a low concentration of EDTA.

\footnotetext{
${ }^{82}$ Images reprinted from [92] with the permission of the American Chemical Society. 


\subsubsection{Functionalization of membranes with disaccharides derived from Microciona prolifera}

Since covalent attachment of sugar molecules to the bilayer was not detectable optically due to similar refractive indices of water and sugar molecules, a competition experiment with the well-detectable peptide $\mathrm{H}^{*}$ was carried out (Figure 3.19 in chapter 3.6.2).

As we were not able to detect the disaccharide binding directly due to its refractive index being too close to the index of water (Figure 5.16, segment 4) we used reflectometry interference spectroscopy (RIfS) to quantify the reduced amount of maleimide groups accessible for covalent binding of $\mathrm{H} 6^{*}$ after running the reaction with the sulfated disaccharide compound.

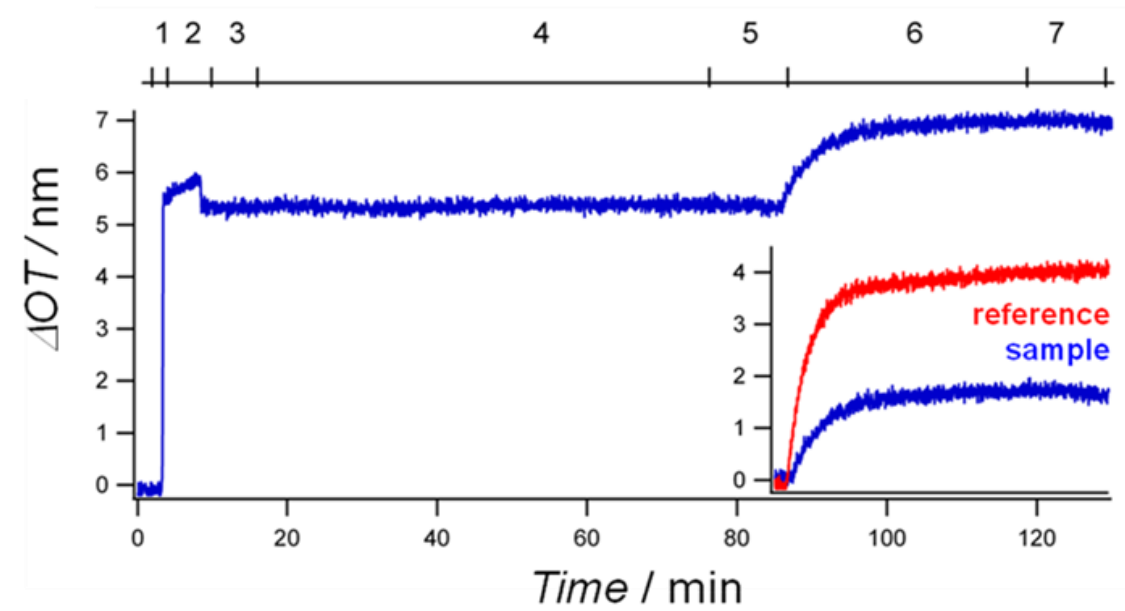

Figure 5.16: Indirect detection of disaccharide binding with RIfS. ${ }^{[92], 82,83,}$ The quantity of remaining binding sites after addition of sulfated disaccharide was determined through binding of Ac-(H)6WGC (H6*) structures. 1) Rinsing with HEPES/Ca ${ }^{2+}$. 2) Formation of POPC bilayer (POPC/MCC-DOPE 90/10). 3) Rinsing with HEPES/EDTA-5. 4) Addition of disaccharide 2. 5) Rinsing. 6) Binding of H6*. 7) Rinsing. Inset: Comparison of the signal after addition and binding of $\mathrm{H6}^{*}$ (blue curve) and reference signal recorded from a bilayer that was not previously loaded with sulfated disaccharide (red curve).

We found that after addition of the sulfated disaccharide after $\sim 83$ minutes (segment 6 ) only $50 \%$ of the maleimide groups were accessible for $\mathrm{H}^{*}$ peptides $(\mathrm{H} 6 *$ peptide binding characterized by increase in $O T$ ).

\footnotetext{
${ }^{83}$ RIfs measurements and data analysis were performed by Milena Stephan, University of Göttingen 


\subsubsection{Necessity of the sulfated epitope and of calcium ions for the self- recognition process}

Figure 5.17 shows a compilation of typical force retraction curves obtained from colloidal probe microscopy. Membrane-coated silicate spheres were brought in contact for a dwell time of $1 \mathrm{~s}$ and subsequently retracted from the surface Figure 5.17-A-C). Interaction forces in the absence of disaccharides attached to the SSLB exhibit a rupture force of $25-50 \mathrm{pN}$ that is entirely abolished in the presence of EDTA-containing buffer Figure 5.17-A). We attribute the background force to calcium-mediated interaction between the choline headgroups.
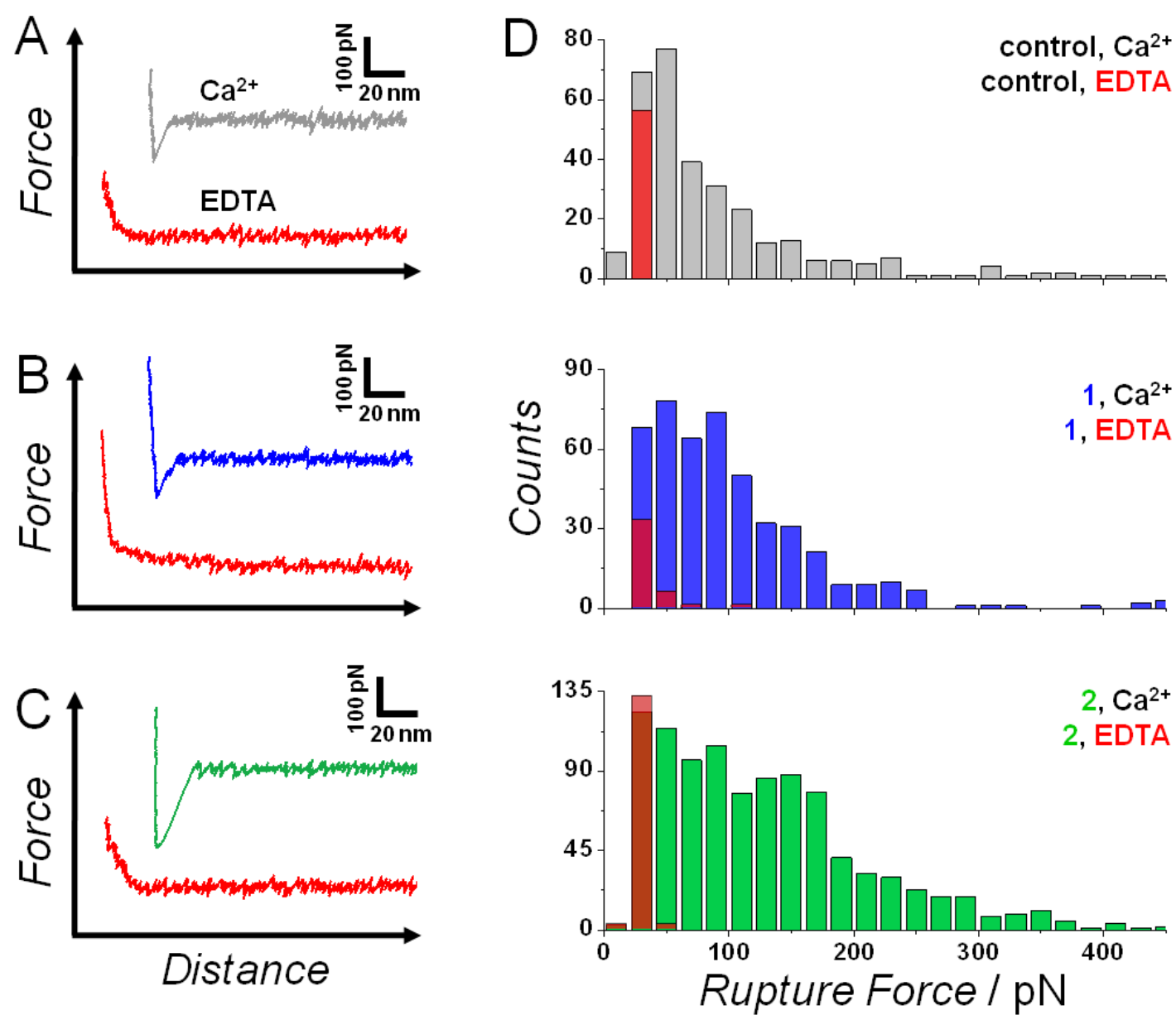

Figure 5.17: Typical force retraction curves of membrane-coated colloidal probes with different functionalization $(A-C)$ and corresponding rupture force histograms (D). ${ }^{[92], 82}$ A: No disaccharide epitopes, B: the non-sulfated disaccharide, and C: the sulfated disaccharide attached to maleimide-bearing phospholipids (10 $\mathrm{mol} \%)$ in a DPPC (substrate) and POPC (probe) matrix. Interactions were probed in calcium buffer before (grey) and after functionalization of membranes with the non-sulfated disaccharide (blue) and the sulfated one (green), respectively. Force curves/data obtained in EDTA containing buffer is shown in red. Measurements were performed at a loading rate of $10 \mathrm{nN} / \mathrm{s}, 1 \mathrm{~s}$ dwell time, and a load force of $200 \mathrm{pN}$ prior to retraction of the cantilever. 
Attachment of non-sulfated disaccharide to the lipid bilayers did only lead to a small shift in interaction force compared to that in the absence of sugar epitopes (Figure 5.17-D). In contrast, coupling of sulfated disaccharides to maleimide groups of the outer membrane leaflets resulted in a shift to larger force that was entirely dependent on the presence of $\mathrm{Ca}^{2+}$. In the presence of the sulfated disaccharide, membrane tethers were frequently formed. Addition of $\mathrm{Ca}^{2+}$ chelators such as EDTA abolished any attractive interaction in all cases (red semitransparent histograms). The histogram of forces found for the homomeric interaction between the sulfated disaccharide in the presence of $10 \mathrm{mM} \mathrm{Ca}^{2+}$ displays several resolvable peaks that we attribute to attractive background interaction between the bilayers at low forces and the disruption of nanoclusters of various sizes at higher forces. These data establish the necessity of the sulfato group for self-recognition, and the essential role of $\mathrm{Ca}^{2+}$ ions in mediating the interactions.

\subsubsection{Kinetics of the disaccharide interaction and cluster formation}

Rupture force histograms recorded at different loading rates illustrate that the contact zone between the membrane-coated colloidal probe and the SSLB comprises a number of parallel bonds (Figure 5.18-A). The estimation of the exact number of participating bonds requires some general thermodynamic and kinetic considerations.

The formation of nanoclusters composed of more than a single dimeric bond is driven by an intricate competition between enthalpic and entropic contributions on the one hand and kinetic assembly schemes on the other hand. ${ }^{[141-144]}$

Smith and Seifert argued that cluster size is governed by binding enthalpy favoring growing clusters and dispersion driven by gain in entropy. ${ }^{[144,145]}$ In essence, weak bonds with a free energy of only few $k_{B} T$ do not lead to large clusters. Those bonds are formed only by stochastic matching upon contact between substrate and probe while in contrast strong bonds accumulate in the contact zone. Assuming that we deal with weak bonds and only one mobile membrane, accumulation of bonds in the contact zone can be largely excluded. 


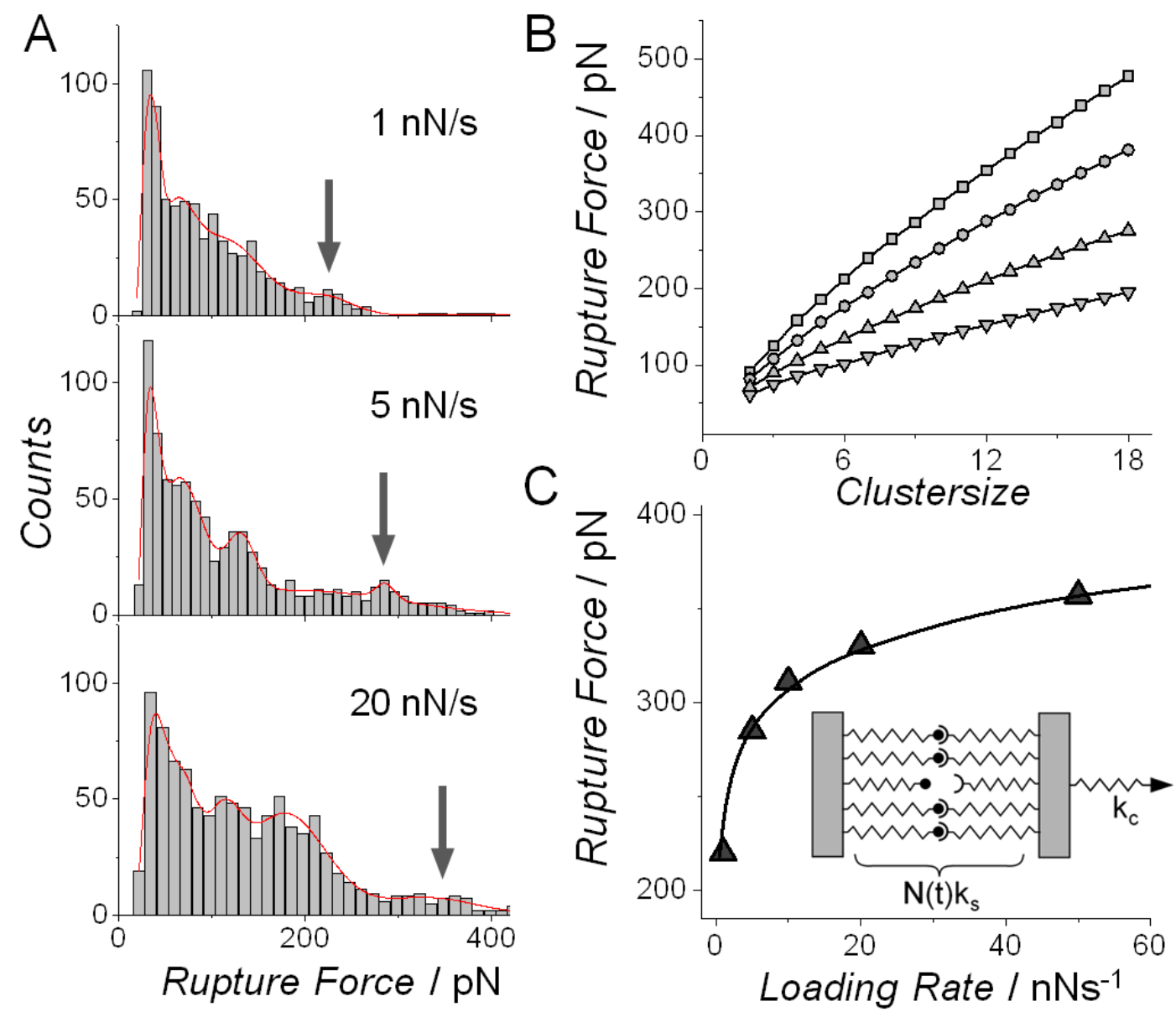

Figure 5.18: Dynamic force spectroscopy of the sulfated disaccharide interaction. A: Rupture force histograms for different loading rates subjected to multipeak fitting of five Gaussian functions to capture the various cluster sizes. ${ }^{[92], 82}$ B: Rupture forces as a function of cluster size obtained by solving the ordinary differential equation of time dependent dissolution of multiple bonds subject to a linear force ramp as a function of bond stiffness: $\boldsymbol{k}_{s}=0.1(\square), 0.025\left({ }^{\circ}\right), 0.01(\Delta)$, and $0.005 \mathrm{~N} \mathrm{~m}^{-1}(\nabla)$. Parameters for modeling: $k_{\text {off }}=0.14 \mathrm{~s}-1, x_{u}=0.36 \mathrm{~nm}, \mu=1 \mathrm{nN} / \mathrm{s}$. C: Rupture force of clusters consisting of $N_{b}=5$ bonds as a function of loading rate $(\Delta)$ subject to fitting the parameters of Equation 10 represented by the solid line. Assumed parameters: $k_{\text {on }}=0, k_{\text {off }}=0.0015 \mathrm{~s}^{-1}, k_{c}=0.01 \mathrm{~N} / \mathrm{m}, k_{s}=3.4 \mathrm{mN} / \mathrm{m}, x_{u}=0.25 \mathrm{~nm}$.

A simple kinetic model introduced by Bell and further investigated by Seifert can be used to describe the rupture of multiple bonds under dynamic load. ${ }^{[95,131,146,147]}$ The rate equation for the time-dependent decrease in the number of bonds $N(t)$ from initially $N_{b}$ reads

$$
\partial t N=-N(t) k_{\text {off }} * \exp \left(\frac{F(t) x_{u}}{k_{b} T}\right)+\left(N_{b}-N(t)\right) k_{\text {on }}
$$

Equation 13

with the dissociation rate $k_{\text {off, }}$ the association rate $k_{\text {on }}$ in equilibrium, and the force per bond $F(t)=k_{\text {sys }} v t=\mu t$, with the pulling velocity $v$ and $\mu$, the loading rate. ${ }^{[147]}$ 
The spring constant, $k_{\text {sys }}=k_{c} k_{s} /\left(N(t) k_{s}+k_{c}\right)$, depends on the spring constant of the cantilever $k_{c}$ and that produced by the involved molecules $\mathrm{N}(\mathrm{t}) \mathrm{ks}$, with $\mathrm{ks}$ the stiffness of a singlebonded molecule. The rupture force of this deterministic model is defined by $F_{\text {rup }}=\mu \tau$, with $\tau$ the lifetime of the cluster defined by $N(\tau)=1$. Equation 10 is solved numerically with initial condition $N(t=0)=N_{b}$. The strategy to obtain as many intrinsic parameters of the system from force spectroscopy works as follows. First, the number of bonds involved in the colloidal probe measurement is estimated from measuring the mean stiffness of the clusters and its variance. Alternatively, counting the number of maxima occurring in a rupture force histogram also serves this purpose (vide infra). The former method also provides the molecular stiffness $k_{s}$ of a single-bonded molecule. The second step comprises rupture force measurements as a function of loading rate to determine the equilibrium off-rate $k_{\text {off }}$ at zero load and the distance between barrier and bound state distance, $x_{u}$. Therefore, these two parameters contained in Equation 10 are fitted to the mean rupture force of a given cluster size as a function of loading rate (Figure 5.18-C). Figure 5.18-A shows the impact of loading rate on the rupture force. As expected, the rupture forces shift to larger values with higher pulling velocity. Importantly, the deterministic model predicts that rupture forces do not linearly increase with cluster size as expected from a simple superposition of parallel springs carrying a shared load. In fact, softer molecular springs with lower $k_{s}$-values produce less dynamic strength $F_{\text {rup }}$ for a given cluster size (Figure 5.18-B). This behavior can be rationalized by considering two limiting cases in current force experiments in which the distance and not the force is controlled. Seifert distinguishes shared load in which $k_{s} \gg k_{c}$ and the force per bond depends on the $N(t)$ existing bonds from the non-cooperative case where $N_{b} k_{s} \ll k_{c}$ and the force per bond is independent of $N(t) .{ }^{[147,148]}$ It is therefore mandatory to estimate the molecular stiffness prior to a detailed analysis of the dynamic strength. We computed $\left\langle N_{b} k_{s}\right\rangle$ from $>400$ force curves and by assuming a Poisson distribution of $k_{s}$ used the identity $k_{s}=$ $\sigma 2 /\left\langle N_{b} k_{s}\right\rangle$ to determine $k_{s}=3.4 \mathrm{mN} / \mathrm{m}$ and $N_{b} \approx 4$. According to the rupture force histograms that were best described by five Gaussians (Figure 5.18-A), we assigned cluster sizes comprising three to five sugar-sugar bonds. This is in good accordance to estimates from Poisson statistics of $k_{s}$. A third way to guess the initial cluster size very coarsely is to divide the measured rupture force of the cluster by the rupture force of a single bond. De Souza measured 
an average rupture force of $30 \pm 6 \mathrm{pN}$ for the individual bond between two sulfated disaccharides in the presence of calcium, which corresponds to approximately seven to eight bonds participating in the cluster. ${ }^{[85]}$ In the following analysis we assume $N_{b}=5$ as derived from the rupture force histograms. Figure $5.18-\mathrm{C}$ shows a fit of to the dynamic strength of the largest clusters comprising five parallel bonds identified from the force histograms.

The experimental data could be best described by an equilibrium off-rate of $\mathrm{k}_{\text {off }} \approx 0.0015 \mathrm{~s}^{-1}$, and a potential width of $x_{u}=0.25 \mathrm{~nm}$ that are in good accordance with force spectroscopy data obtained from Anselmetti and co-workers measuring the dynamic strength of a single g-200/g-200 interaction. ${ }^{[87]}$ The potential width is slightly closer to the barrier than predicted $\left(x_{u}=0.35 \mathrm{~nm}\right)$. The loss in strength due to a closer distance to the barrier might be rationalized by the more complex oligosaccharides involved in the homomeric g-200 interaction rendering the deviation within a reasonable range.

Considering the rather large membrane-coated probe used in our study with a radius of 7.5 $\mu \mathrm{m}$, the dynamic strength of only very few bonds are assessed after a dwell time of $1 \mathrm{~s}$. We attribute this to a combination of various contributions. The effective contact area is very small considering that only those bonds carry the load efficiently that reside at the bottom of the potential, which is in the center of the adhesion zone. This effect is enhanced by the finite surface roughness of the probe $(r m s \sim 0.7 \mathrm{~nm}) .{ }^{[87]}$ Due to the inherent curvature of the probe, bonds are only formed at a distance of $70 \mathrm{~nm}$ from the center of the sphere. Taking into account that $k_{\text {off }}$ increases exponentially with distance from the center of the spherical probe only few bonds effectively participate in a cluster. Enrichment of bonds in the contact zone is largely prohibited by entropy costs due to the low binding energy of the homodimers. Besides, the non-bound sugar moieties act as repeller molecules that produce repulsion upon close contact. Due to the finite softness of the bound molecules, the rupture force becomes less strongly influenced by the number of bonds constituting the cluster, which eventually leads to a systematic underestimation of $N_{b}$. In the limiting case of a very soft linker it is conceivable that rupture forces of clusters are independent of the number of participating bonds. 


\subsubsection{Membrane probe spectroscopy of weak disaccharide interactions}

In order to mimic aspects of the cellular glycocalyx, we investigated the membrane probe system equipped with carbohydrate structures derived from the marine sponge Microciona prolifera, which are reported to perform a $\mathrm{Ca}^{2+}$-dependent self-recognition process. The multivalent carbohydrate-carbohydrate interaction between membrane-anchored epitopes has been explored after in situ coupling of sulfated and non-sulfated disaccharides to membrane-coated surfaces. Force spectroscopy measurements were employed to mimic native cell-cell contacts and to extract rupture force values as well as kinetic parameters. The dynamic strength of the homomeric self-association was measured as a function of calcium ions and loading rate. A deterministic model was used to estimate the basic energy landscape and number of participating bonds in the contact zone.

In conclusion, we investigated the formation and dynamic strength defined as the rupture force of carbohydrate nanoclusters between two opposing membranes equipped with disaccharide epitopes derived from the marine sponge M. prolifera. The weak binding affinity prevents clustering beyond statistical matching and therefore allows observing the bond breakage from individual bonds to very small ensembles comprising only few dimers. Coupling of the epitopes was quantified by reflectometric interference spectroscopy (RIfS), revealing a coupling efficiency of $50 \%$ for 2 . The presented data establish the necessity of the sulfato group for self-recognition, and the essential role of $\mathrm{Ca}^{2+}$ ions in mediating the interactions. 


\subsection{Coiled-coil formation of $i$-E3Cys/i-K3Cys lipopeptides}

\subsubsection{Probing the $i$-E3Cys/i-K3Cys interaction with a membrane probe setup} The heterodimeric interaction between $i$-E3Cys and $i$-K3Cys peptides was studied on POPC matrices with $0.1,1,3$, and 10 mol\% of maleimide-bearing lipids. ${ }^{[96]}$ POPC was chosen to allow for diffusion of lipopeptides during the experiments, which were carried out at room temperature. In all measurements, the colloidal probe was equipped with $i$-K3Cys after incubation with POPC vesicles, whereas the membrane on the flat support was equipped with $i$ E3Cys peptides. We used colloidal probe cantilevers with spring constants of $k_{c}=0.01 \mathrm{~N} / \mathrm{m}$, load forces of $200 \mathrm{pN}$, dwell times ranging from a few milliseconds to $0.5,1$, 2, or 5 seconds, and varying pulling velocities to get a detailed analysis of the dynamic strength of the heterodimeric coiled-coil system.

\subsubsection{Functionalization of membranes with $i$-E3Cys and $i$-K3Cys}

Ellipsometry was used to control the immobilization of the peptides on the membrane surfaces.$^{84}$ As depicted in Figure 5.19, the maleimide-functionalized vesicles readily spread on the silicon wafer characterized by a decrease of the angle del (red line) by about $3.5^{\circ}$. A total concentration of $100 \mathrm{nmol} / \mathrm{mL}$ peptide (two additions of peptide, $i$-E3Cys- 1 and $i-E 3 C y s-2$ in graph) in the closed loop liquid handling system is needed to initialize the binding of $i$-E3Cys to the maleimide-functionalized membrane after rinsing the system.

After saturation is reached, unbound peptides are removed by circling buffer through the system, before $i$-K3Cys-labeled SUV are coupled to the $i$-E3Cys-functionalized layer. The strong decrease in del represents the increase in layer thickness by vesicle binding. Rinsing did not lead to a decrease in layer thickness as it would be the case during detachment of peptides or peptide-functionalized vesicles.

\footnotetext{
${ }^{84}$ Measurements were carried out with the support of Gesa Pähler, University of Göttingen. 


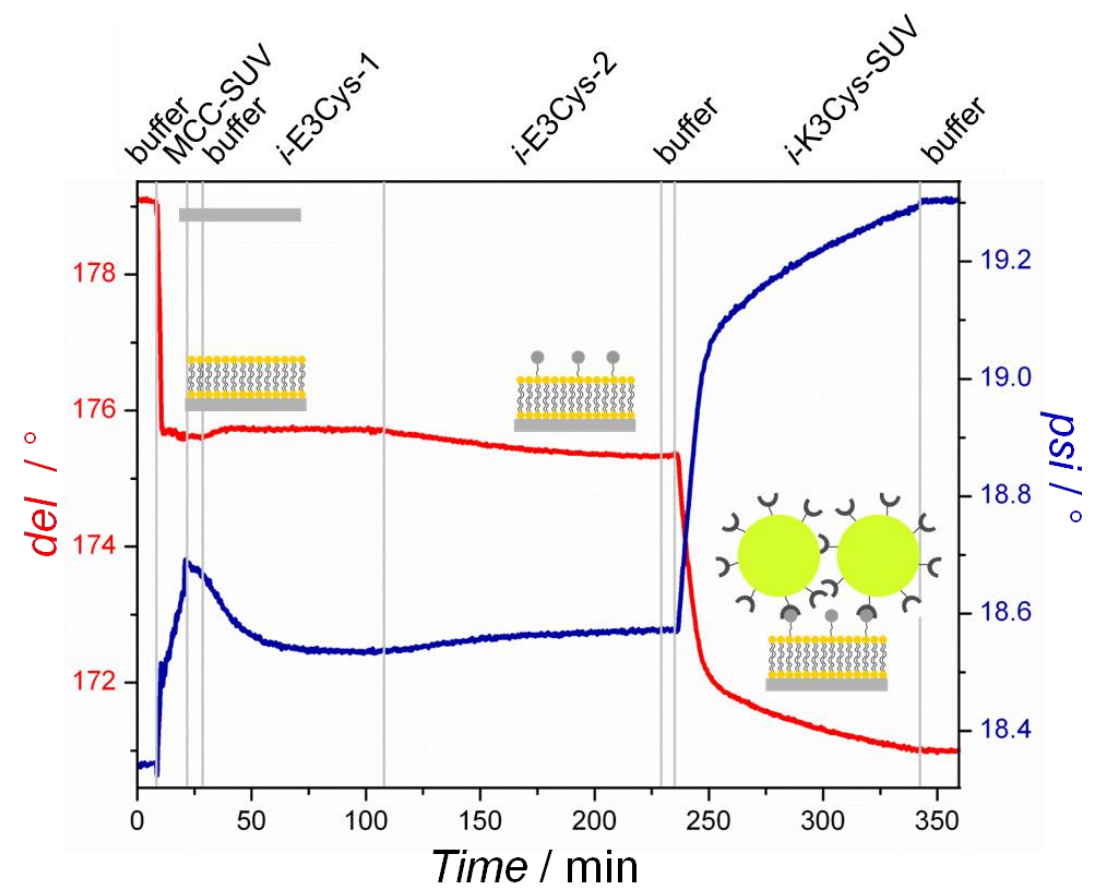

Figure 5.19: Functionalization of a silicon substrate with a POPC membrane, $i$-E3Cys peptides, and $i$-K3Cysfunctionalized vesicles as monitored by ellipsometry. Graph shows angles del and psi corresponding to the increase in thickness of optical layers. A maleimide tagged lipid bilayer is formed by vesicle spreading (first drop of del signal) and the bilayer is functionalized by addition of $i$-E3Cys $(100 \mathrm{nmol} / \mathrm{mL})$. The shallow decrease of del recorded after 100 minutes indicates $i$-E3Cys binding. Addition of $i$-K3Cys-functionalized vesicles results in a strong decrease of del corresponding to a pronounced increase in layer thickness due to the adsorption of functionalized vesicles.

In control measurements carried out with membranes lacking maleimide-functionalized lipids, $i$-E3Cys peptides did not bind to the membranes, whereas $i$-K3Cys peptides adsorbed to the membrane and were washed off the membrane when buffer was circled through. ${ }^{[149]}$ Hence, we conclude that the peptides reliably bind to maleimide-functionalized membranes via their terminal cystein residue. ${ }^{[95]}$ Besides ellipsometry, ATR-FTIR measurements were used to confirm the binding of cystein-tagged peptides to maleimide-functionalized membranes. ${ }^{[149]}$ Control measurements and ATR-FTIR measurement were carried out by G. Pähler. ${ }^{[95]}$

\subsubsection{Impact of lipopeptide concentration, dwell time, and loading rate on interaction forces between $i$-E3Cys/ $i$-K3Cys-functionalized membranes}

Membrane probe spectroscopy measurements were used to detect interaction forces between membranes decorated with $i$-E3Cys and $i$-K3Cys peptides, respectively. ${ }^{[96]}$ We found 
that membrane interaction forces are significantly increased on peptide-functionalized membranes in contrast to non-functionalized ones, which show most probable rupture forces under $50 \mathrm{pN}$ (grey curve in Figure 5.20-A). The membrane interaction forces at a pulling velocity of $1 \mu \mathrm{m} / \mathrm{s}$ are characterized by a multipeak force profile (Figure 5.20-B) and range from $\approx 80 \mathrm{pN}$ to $\approx 600 \mathrm{pN}$.
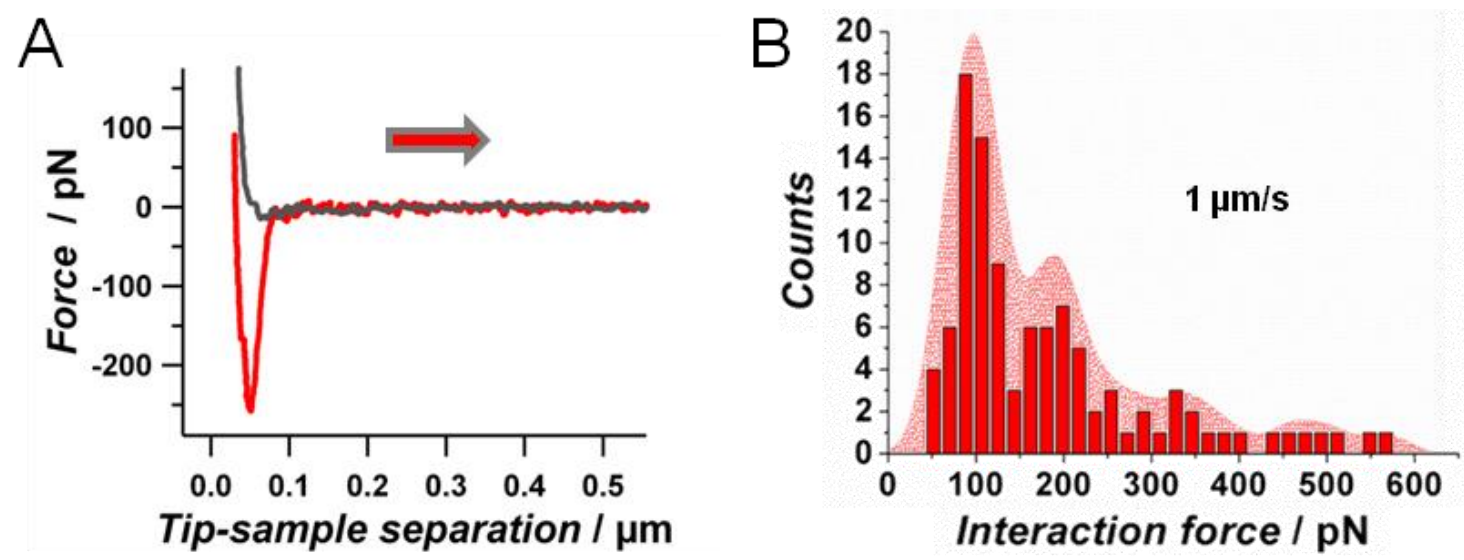

Figure 5.20: Interaction forces as recorded for the $i$-E3Cys/i-K3Cys interaction. ${ }^{[96], 85} \mathrm{~A}$ : Force retraction curves at a pulling velocity of $1000 \mathrm{~nm} / \mathrm{s}$ for $3 \mathrm{~mol} \%$ of lipopeptides (red curve) and for neat membranes (grey curve). In the presence of $i$-E3Cys/i-K3Cys lipopeptides in the membranes, interaction forces are significantly higher $(\approx 250 \mathrm{pN})$ than in the case of unfunctionalized membranes $(\approx 20 \mathrm{pN})$. B: In the presence of the lipopeptides, we observed a multi-peak profile with a most probable rupture force of $\approx 100 \mathrm{pN}$ ( $3 \mathrm{~mol} \%$ peptides, $1 \mu \mathrm{m} / \mathrm{s}$ velocity, $k_{c} \approx 0.01 \mathrm{~N} / \mathrm{m}$ ).

However, if compared to calculations involving the number of available peptide structures in the contact zone and the estimated strength of the peptide interaction, the interaction forces are substantially lower than expected. As determined by Pähler et al., the free energy of the coiled-coil interaction is $10.6 k_{B} T$, which equals an interaction force of $17 \mathrm{pN}$ at a helix length of $2.7 \mathrm{~nm} \cdot{ }^{[149,150]}$ Rief and coworkers determined the unzipping forces of coiled-coil structures to be $\approx 12 \mathrm{pN}$ being rather independent of the loading rate. If assuming a contact area of $0.011 \mu^{2}$, a Young's modulus of the system of $10 \mathrm{MPa}$, an area per lipid of $0.7 \mathrm{~nm}^{2}$, and a mol percentage of available peptides on the surface of $3 \%$, we end up with a number of 470 lipopeptides, which can participate in the interactions.

\footnotetext{
${ }^{85}$ Images reprinted from [96] with the permission of Elsevier.
} 
If accounting for a single coiled-coil interaction force of $17 \mathrm{pN}$, we end up with overall interaction forces of around $8 \mathrm{nN}$ in the contact area, a value, which is eight times higher than the highest force measurement with this setup. Therefore, we conclude that not all peptide structures on the surface are available for binding.

To clarify this, FRAP measurements as well as AFM imaging of lipopeptide-functionalized membranes were carried out showing that $i$-E3Cys and $i$-K3Cys lipopeptides form patterns on the membrane surface. ${ }^{86}$ In contrast to $i$-E3Cys structures, the well-defined borders of $i$-K3Cys clusters allowed for an estimation of the equivalent disc radius of the cluster. A mean radius of $(21 \pm 8) \mathrm{nm}$ was found. FRAP analysis showed that the diffusion coefficient of $i$-K3Cysfunctionalized membranes (10 mol\%) decreased by a factor of two $\left(D=(2.5 \pm 1.6) \mu \mathrm{m}^{2} / \mathrm{s}\right)$ compared to unfunctionalized membranes $\left(D=(5.5 \pm 2.5) \mu \mathrm{m}^{2} / \mathrm{s}\right) \cdot{ }^{[96]}$ Coupling of $i$-E3Cys peptides to the $i$-K3Cys-functionalized membrane further reduced the diffusion coefficient to a value below $1 \mu \mathrm{m}^{2} / \mathrm{s}$. After labelling the peptide structures fluorescently we found a similar trend in diffusion coefficients as well as a decrease in the mobile fraction of fluorescent structures. This led us to the assumption that coiled-coil lipopeptides are immobilized in small clusters leading to reduced membrane interaction force values. As the membrane probe is repositioned between individual measurements, it seems reasonable to assign the multiple peaks in the force distribution depicted in Figure 5.20-B to several lipopeptide clusters involved in the membrane interaction processes during a set of experiments.

It is conceivable that the lateral organization and the number of clusters in the contact area influence the magnitude of forces. To further quantify this effect, we performed membrane probe spectroscopy measurements with differing maleimide concentration, dwell time, and pulling velocity. ${ }^{86}$ We found that interaction forces non-linearly increase from a mean force of $\approx 80 \mathrm{pN}$ to a mean force of $\approx 550 \mathrm{pN}$ upon increasing the lipopeptide concentration in the membrane up to $10 \mathrm{~mol} \%$ ( $1 \mathrm{~s}$ dwell time, $1 \mu \mathrm{m} / \mathrm{s}$ pulling velocity, Figure $5.21-\mathrm{A}$ ). The fact that the interaction forces at $10 \mathrm{~mol} \%$ are lower than expected is attributed to a progressive steric hindrance of peptide interactions on the surface.

\footnotetext{
${ }^{86}$ More information can be found in 96. Lorenz, B., et al. (2013). "Impact of peptide clustering on unbinding forces in the context of fusion mimetics." Biochem. Biophys. Res. Commun. 430(3): 938-943.
} 
Upon an increase of the dwell time up to five seconds, we observed an increase of median forces from $\approx 40 \mathrm{pN}$ to median forces of $\approx 200 \mathrm{pN}$ at a pulling speed of $1 \mu \mathrm{m} / \mathrm{s}$ and a concentration of lipopeptides of 3 mol\% (Figure 5.21-B). The effect of the dwell time on the interaction forces might be limited due to the reduced diffusion of lipopeptides in the membrane.
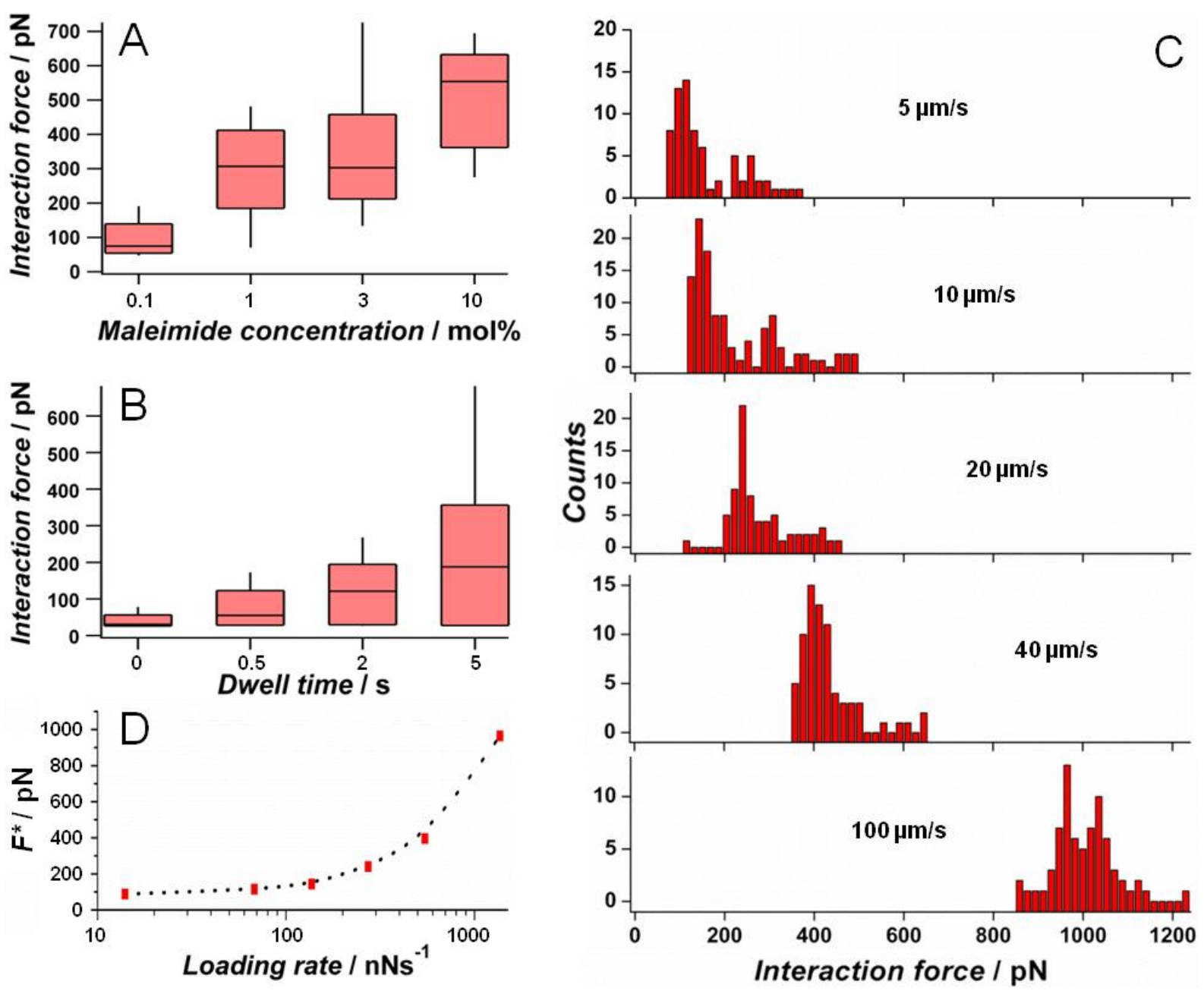

Figure 5.21: Impact of maleimide concentration, dwell time, and loading rate on interaction forces between $i$-E3Cys and $i$-K3Cys-functionalized opposing membranes at a load force of $200 \mathrm{pN} .{ }^{[96], 85} \mathrm{~A}$ : A nonlinear increase in interaction forces from mean values of $\approx 80 \mathrm{pN}$ to $\approx 550 \mathrm{pN}$ was detected upon an increase of lipopeptides from $0.1 \mathrm{~mol} \%$ to $10 \mathrm{~mol} \%$ ( $1 \mathrm{~s}$ dwell time, 1 rm/s pulling velocity). B: The increase of dwell time from a few milliseconds (" $0 \mathrm{~s}$ ") to five seconds leads to an increase in interaction forces from a median of $\approx 40 \mathrm{pN}$ to a median of $\approx 200 \mathrm{pN}$ (1 $\mu \mathrm{m} / \mathrm{s}$ pulling velocity, $3 \mathrm{~mol} \%$ lipopeptides). C: Loadingrate dependent measurements reveal that the interaction forces are velocity-dependent at pulling speeds higher than $20 \mu \mathrm{m} / \mathrm{s}$ ( $1 \mathrm{~s}$ dwell time, $3 \mathrm{~mol} \%$ lipopeptides). Interaction forces range from $\approx 100 \mathrm{pN}$ at $5 \mu \mathrm{m} / \mathrm{s}$ to $\approx 1000 \mathrm{pN}$ at $100 \mu \mathrm{m} / \mathrm{s}$. The multiple peaks are assigned to different lipopeptide clusters taking part in the membrane interaction. D: Plotting of the most probable rupture forces of C logarithmically against the loading rate confirms that the interaction forces are rather velocity-independent at rates below $200 \mathrm{nNs}^{-1}$. 
As depicted in the different histograms in Figure 5.21-C, velocity-dependent measurements revealed that the membrane interaction forces mediated by $i$-E3Cys and $i$-K3Cys are rather velocity-independent up to a velocity of $20 \mu \mathrm{m} / \mathrm{s}$ and that non-equilibrium conditions are only reached at loading rates higher than that value equaling to a loading rate of about $150 \mathrm{nN} / \mathrm{s}$ (Figure 5.21-D).

5.5.4 Estimation of the number of $i$-E3Cys/ $i$-K3Cys coiled-coils involved in the contact zone

The distinct force profiles lead to the assumption that each peak can be assigned to one cluster and that a maximum of five clusters is involved in the measurement (Figure 5.20). ${ }^{[96]}$ To evaluate how many heterodimers are involved in each peak, it is important to know about the strength of an individual i-E3Cys/i-K3Cys lipopeptide interaction. By using 0.1 mol\% lipopeptides in the membranes, we were able to identify unbinding events, which were characterized by forces down to $25 \mathrm{pN}$ and a shape following the worm-like chain model (Figure 5.22-A). This led us to the assumption that we detected the unbinding of a single pair of coiled-coil peptides. This means that the cluster assigned to the peak at $100 \mathrm{pN}$ (Figure 5.20-B) is characterized by four individual coiled-coil interactions. The second and third peak in Figure 5.20B would then be composed of 8 and 14 interactions.
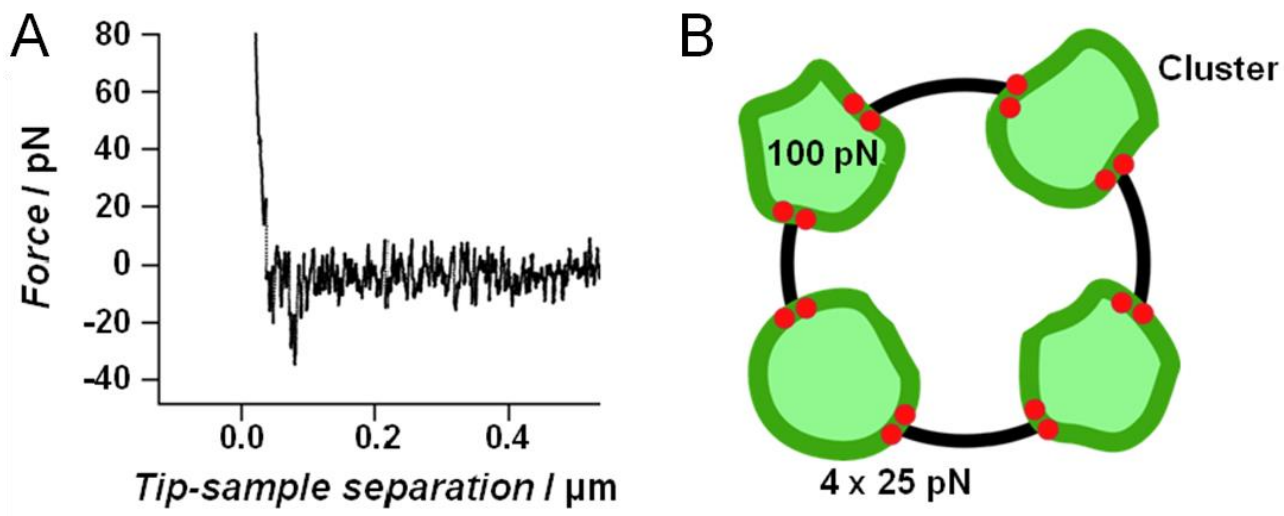

Figure 5.22: The number of $i$-E3Cys/i-K3Cys interactions involved in membrane probe experiments. ${ }^{[96], 85}$ The lowest detectable $i$-E3Cys/i-K3Cys interaction force scales around $25 \mathrm{pN}$ and is extracted from the worm-like chain like unbinding event depicted in A. Due to the inactivation of peptides inside an $i$-K3Cys cluster on the probe, only peptides in the intersection between the outer cluster shell and the outer border of the contact area are assumed to undergo coiled-coil formation (B). 
However, if the $i$-E3Cys/i-K3Cys coiled-coil interaction is characterized by a lower individual force, the number of peptides involved during a set of measurements increases if calculated from the most probable rupture force peaks. With an interaction force of $17 \mathrm{pN}$, the clusters mentioned above would be made up from around 6, 12, and 20 peptide interactions, respectively.

Judging from our measurements, the large difference to the calculated 470 peptide interactions in the contact area can be explained by three factors: 1) The sterical hindrance of peptides, which are embedded inside the clusters leads to a reduction of interaction forces. We found that only $18 \%$ of peptides at the border of the cluster are available for binding. 2) Moreover, the number of clusters in the contact area of the membrane probe is limited to an average of 1.6 (calculated from the number of clusters per $\mu \mathrm{m}^{2}$ and the estimated size of the contact zone) ${ }^{[96]}$ Due to the inactivation of $i$-K3Cys peptides inside the clusters on the membrane probe, the number of possible interactions between $i$-K3Cys peptides in the outer shell of a cluster and the $i$-E3Cys peptides on the second membrane (red dots in Figure 5.22-B) may be limited to the intersection between cluster shell (green line) and the border of the contact area (black line). 3) Finally, the roughness of the glass bead serving as the colloidal probe can reduce the contact area of probe and sample and thereby diminish the number of coiled-coil units formed between the membranes. ${ }^{[107]}$

\subsubsection{Impact of peptide clustering on membrane docking efficiency}

While studying $i$-E3Cys/i-K3Cys lipopeptide interactions in POPC membranes, we found that lipopeptides assemble into clusters with a radius of around $22 \mathrm{~nm}$. Lang et al. showed in 2001 , that syntaxin forms cholesterol-dependent nanodomains with a size of 50 to $60 \mathrm{~nm}$. $^{[151 \text {, }}$ ${ }^{152]}$ Liu et al. were able to show that an increase in protein concentration by a factor of 100 leads to a reduced docking and thus fusion efficiency. ${ }^{[153]}$ This is in good accordance to the findings of Pähler et al. where the fusion efficiency for the analyzed peptide system was found to be $18 \%$ for hemi- and only $3 \%$ for full fusion using bulk fusion assays with single unilamellar vesicles. ${ }^{[95]} \mathrm{A}$ high surface concentration and the steric hindrance of lipopeptides by clustering might be the explanation for the lack in fusion efficiency in artificial systems. In 
contrast, the zipping process of native SNARE proteins seems to provide the free energy required to overcome the fusion barrier. In contrast to the peptides used in this study, SNARE structures are able to form long superhelices. As lateral clustering reduces the membrane docking efficiency in our experiments, we assume that the length of the helices is the parameter critical for mediating membrane fusion. 
A Force Spectroscopy Setup to Mimic Cellular Interaction Processes 


\section{Towards cellular interactions:}

\section{Single molecule force spectroscopy of cadherin-mediated interactions}

\subsection{Characterization of cadherin-functionalized surfaces}

The site-specific immobilization of cadherin constructs on surfaces is crucial for successful force spectroscopy of the cadherin-cadherin interaction. Hence, a reliable and functional immobilization of proteins is essential. As described in chapter 3.8.2, AFM imaging and antibody staining has been used to control the deposition of cadherins prior to force spectroscopy measurements. Cantilever and substrate were functionalized following the protocols of Engin et al. described in the same chapter. ${ }^{[104]}$ Figure 6.1-A depicts the squared micropatterns that were used to detect and discuss surface coupling of E-cadherin constructs. After coupling EC15 to a BG-functionalized gold surface, the topography of the sample was imaged by AFM.
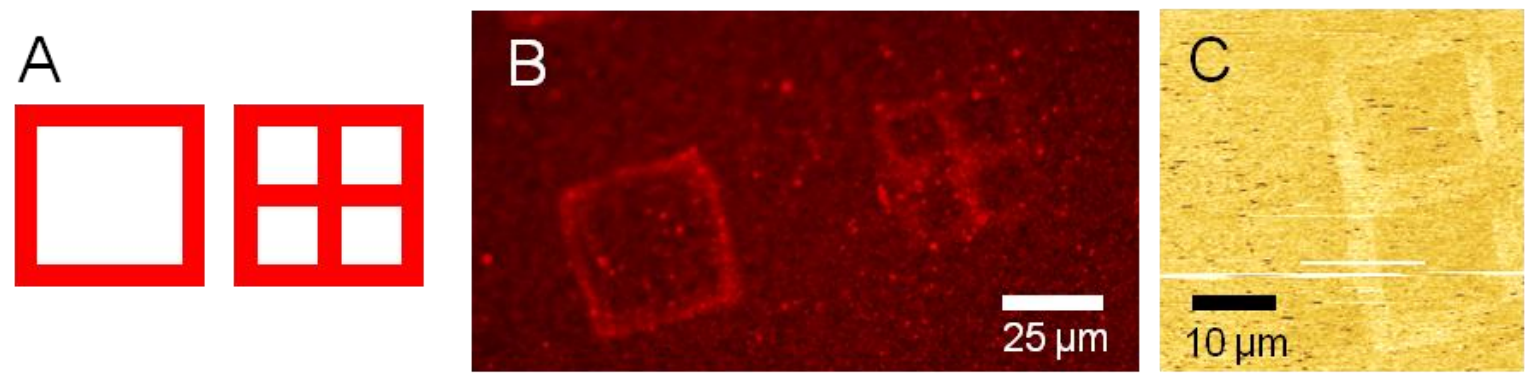

Figure 6.1: Micropatterned BGT/MT surfaces after functionalization with EC15. Window-shaped structures depicted in A were patterned on gold surfaces and subsequently incubated with EC15 constructs. B: Secondary antibody staining approves the functionality of cadherin structures on the surface as high fluorescence intensity is observed on the window-like structures due to the specific binding of first antibody to cadherin constructs and the subsequent binding of fluorescently labeled secondary antibody to the first antibody. Background fluorescence is ascribed to a certain extent of non-specific binding of fluorescent antibody. C: AFM imaging of surfaces reveal the successful immobilization of cadherin structures characterized by a window-shaped elevation.

As displayed in Figure 6.1-B window-shaped elevations of 2 to $4 \mathrm{~nm}$ height difference to the non-coated substrate areas could be detected. Due to the forces exerted on the surface by a cantilever during imaging, ablation of protein material during the measurement is likely resulting in an underestimation of the size of bound protein material. As similar measurements on samples lacking EC15 did not show these structures, we interpret the result as an indication of successful EC15 binding to the surface. 
Additionally, an immunohistochemical assay was employed to determine the functionality of EC15 and EC12 structures immobilized via SNAP sequences. After incubating EC15-patterned surfaces with primary antibody and subsequently with fluorescently labeled secondary antibody, we observed a window-shaped fluorescence (Figure 6.1-C) reproducing the patterns depicted in Figure 6.1-A. Control samples lacking the EC15 incubation step did not show any micropatterns. From these findings, we conclude that we were able to functionally immobilize E-cadherin constructs on BG-functionalized surfaces building the prerequisite for the study of homomeric cadherin interactions in a force spectroscopy setup.

\subsection{Single molecule force spectroscopy of the homomeric cadherin in- teraction}

\subsubsection{Interaction forces between EC15/EC15 and EC12/EC12}

The following section describes results obtained from force spectroscopy experiments of the cadherin constructs EC12 and EC15 after homomeric bond breakage under a linear force ramp. The single-molecule setup established is depicted in Figure 6.2.

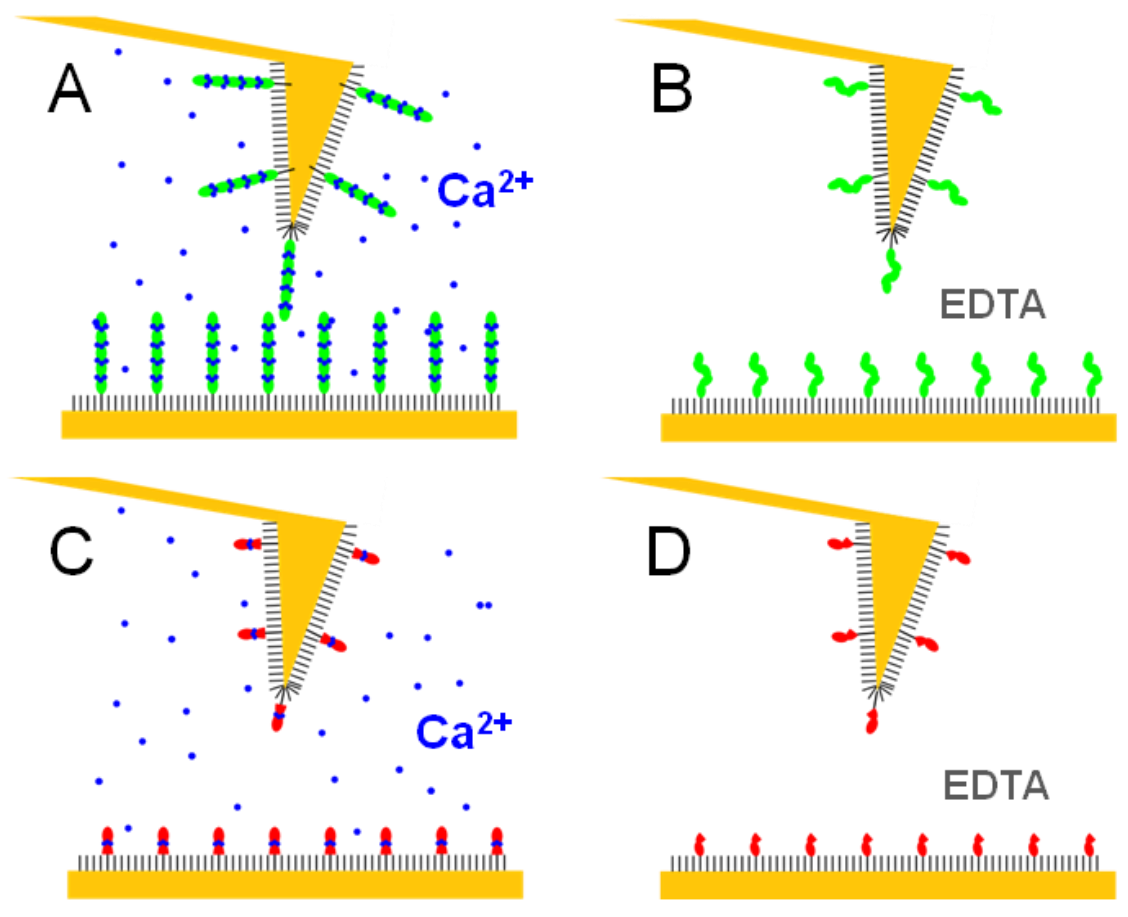

Figure 6.2: Schematic representation of single molecule force spectroscopy to probe homomeric cadherin interactions. A, B: EC15 proteins being probed in the presence (A) and in the absence of $\mathrm{Ca}^{2+}$ ions (B). C, D: Truncated cadherin proteins EC12 being probed in the presence (C) and in the absence of $\mathrm{Ca}^{2+}$ ions (D). 
Interactions were probed between $E C 15$ constructs $(A, B)$ and between $E C 12(C, D)$ constructs in the presence $(A, C)$ and in the absence $(B, D)$ of calcium ions as schematically depicted in Figure 6.2. Figure 6.3 shows histograms of maximum adhesion forces $F_{a d}$ as collected from EC12 (red/grey) and EC15 (green/grey) force-extension measurements. Notably, all adhesion events were compiled in the histogram regardless whether occurring directly after losing contact with the surface without a binding being established between tip and sample or after extending a polymer/polypeptide linker prior to unbinding (vide infra).

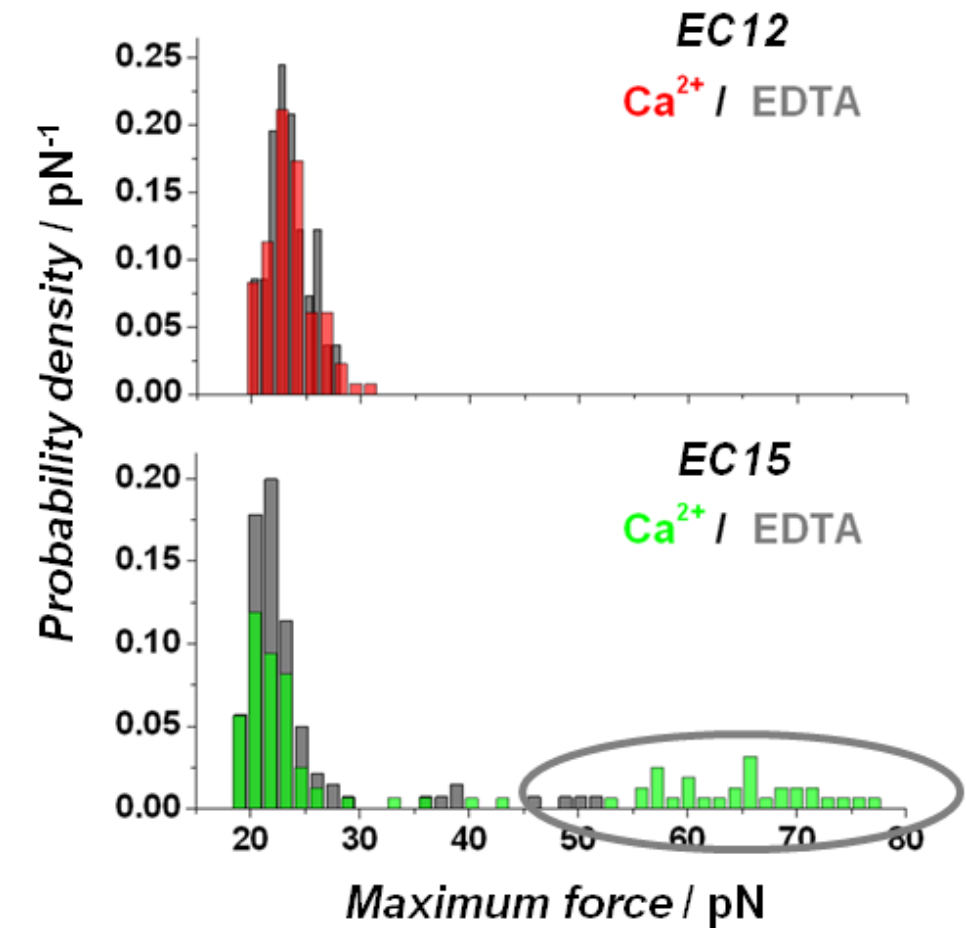

Figure 6.3: Histograms of maximum adhesion forces collected from homomeric EC12 (red/grey) and EC15 (green/grey) measurements. Histograms include maximum force values from each retraction curve no matter if a WLC-like event was detected during the particular force cycle or not. In the case of the EC15/EC15 interaction a second group of events can be detected at forces ranging from 50 to 80 pN, which was subjected to further analysis.

In later refinement of data reduction, only those rupture events were considered, which occur after stretching of a single polymer chain (wormlike chain behavior). This allows us to collect only events that correspond to single molecule mechanics and are not due to non-specific contact mechanics dominated by van-der-Waals forces. In both cases, a high number of adhesion forces occur in the range of 20 to $30 \mathrm{pN}$. However, EC15 measurements yield interaction forces in a higher regime ranging from 50 to 80 pN. Figure 6.4 shows typical force exten- 
sion curves in the presence (red, green) and in the absence (grey) of $\mathrm{Ca}^{2+}$ ions (2 mM) for EC12 (A) constructs and EC15 constructs (B).

The rupture event documents the unbinding of individual bonds as confirmed by WLC-fits (blue line in Figure 6.4-A), which provide persistence lengths of $l_{p}=(0.5 \pm 0.1) \mathrm{nm}$ indicative of single polypeptide chains. ${ }^{[154]}$

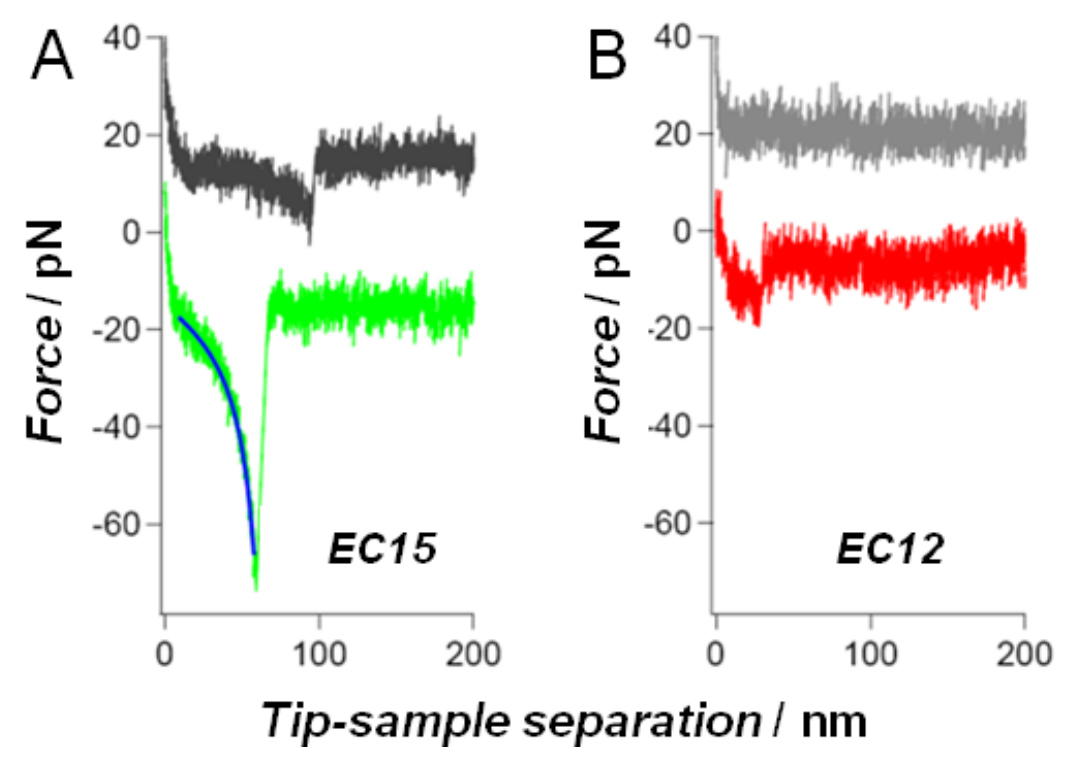

Figure 6.4: Force-retraction curves showing the unbinding of EC15 (left side) and EC12 (right side) cadherin dimers in the presence (red and green) and absence (light and dark grey) of $\mathrm{Ca}^{2+}$ ions. ${ }^{[155]}$ Contact time was $0.1 \mathrm{~s}$, cantilever spring constant $k_{c}=6 \mathrm{pN} / \mathrm{nm}$. A pronounced worm-like chain deflection of the cantilever is observed in the case of EC15 constructs in the presence of calcium ions. In the absence of ions, the shape of the unbinding event is similar to the shape of the event in presence of calcium ions, but rupture forces decreased by a factor of $\sim 5$. Unbinding forces of EC12 constructs are in the range of the baseline noise vanishing completely in the absence of calcium ions.

Generally, unbinding forces of the homomeric EC15 bonds $(35-90 \mathrm{pN})$ in the presence of $\mathrm{Ca}^{2+}$ were substantially larger than those found for EC12 dimers (20-30 pN). Rupture force histograms clearly show that EC15 displays specific interaction with unbinding forces around 35-90 pN depending on the loading rate, while EC12 lacks such high force regime. While addition of EDTA ( $2 \mathrm{mM}$ ) entirely abolishes this specific interaction between EC15 cadherins, virtually no impact of $\mathrm{Ca}^{2+}$ depletion was found for rupturing of $E C 12$ dimers. 


\subsubsection{Contact time dependence of the EC15/EC15 interaction}

Contact time between functionalized AFM tip and substrate was varied from 0 to $5 \mathrm{~s}$ showing an increase in the number of detectable events (Table 1) but no significant impact on unbinding forces. Notably, the number of multiple unbinding events also increased with the contact time on the surface.

\begin{tabular}{c|c} 
Contact time / s & EC15/EC15 events / \% \\
\hline ms regime & 12 \\
\hline 0.5 & 33 \\
\hline 2 & 51 \\
\hline 5 & 56
\end{tabular}

Table 1: Number of unbinding events as a function of contact time. The number of successful EC15/EC15 dejoining events increases with contact time.

\subsubsection{Loading rate dependence of the EC15/EC15 interaction}

The loading rate dependence was only investigated for EC15 dimers that disjoin after nonlinear stretching in a wormlike chain fashion. Non-specific interactions are excluded from the analysis. Besides, shorter EC12 constructs were not part of the analysis due to difficulties in distinguishing between specific and non-specific interactions.

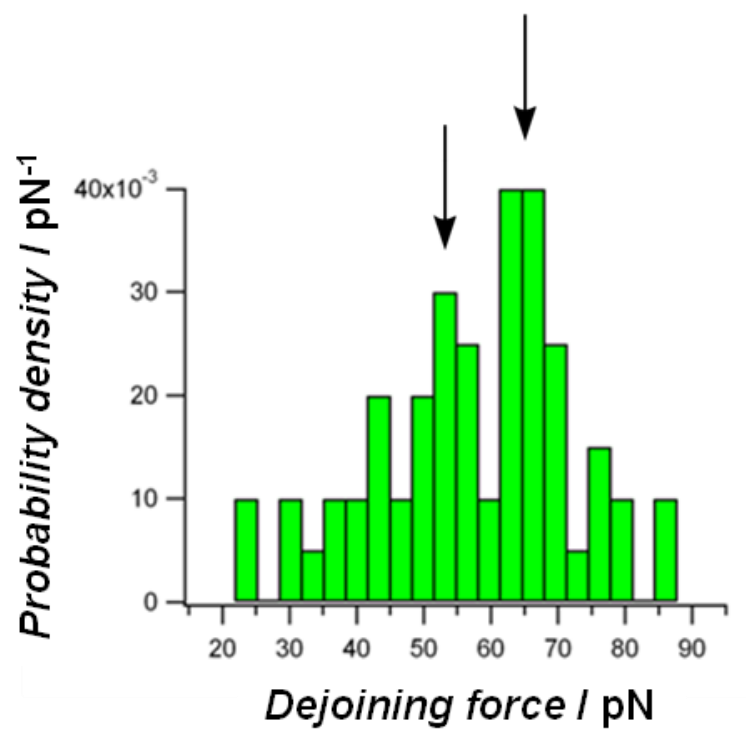

Figure 6.5. Typical rupture force histogram obtained from disjoining of EC15 functionalized surfaces characterized by a worm-like chain like shape of the rupture event (loading rate of $\sim 6 \mathrm{nN} / \mathrm{s}$ ). The histogram is characterized by a bimodal distribution with maxima of probable rupture forces at $55 \mathrm{pN}$ and at $65 \mathrm{pN}$ (indicated with arrows). 
The bond rupture histograms of the specific interaction forces between EC15 reveal a bimodal distribution at all loading rates (Figure 6.5). Plotting the most probable rupture forces as a function of loading rate $r_{f}$ provides a means to compute the off-rate at zero force $k_{\text {off }}(F$ $=0)$ and the distance from the ground state to the transition state $x_{u}$ assuming a single well potential. According to Equation 14 the linear regression of $F_{\text {rup }}$ as a function of $\ln \left\{R_{f}\right\}$ provides both, $x_{u}$ as well as $k_{\text {off }}$.

$$
F_{\text {rup }}=\frac{k_{b} T}{x_{u}} \ln \left\{\frac{x_{u} r_{f}}{k_{b} T k_{\text {off }}}\right\}
$$

Equation 14

We treated the bimodal distribution as if two types of bonds were probed, therefore plotting the maxima of the first (dark grey circles) and second peak (light grey squares) separately (Figure 6.6). For comparison, we also added data taken from Leckband and coworkers (green symbol matching largely our more stable bonds (light grey squares). ${ }^{[101]}$ The less stable bonds (dark grey circles) exhibit $k_{\text {off }}=5^{\star} 10^{-3} \mathrm{~s}^{-1}$, while the more stable ones possess a considerably higher lifetime $\left(k_{\text {off }}=2 \star 10^{-5} \mathrm{~s}^{-1}\right)$. In accordance with Leckband and coworkers the width of the potential-well amounts to $x_{u} \approx 1-1.5 \mathrm{~nm}$.

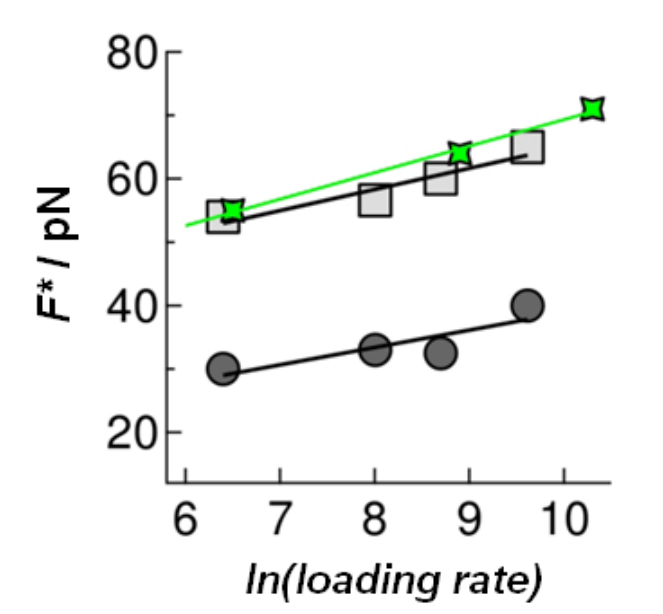

Figure 6.6: Dynamic force spectra of the calcium-dependent EC15/EC15 interaction. ${ }^{87}$ Dark grey cycles represent the dynamic strength according to the first peak of the bimodal rupture force distribution indicated by the left arrow in Figure 6.5, while light grey squares correspond to the second peak. Solid black lines are fits according to Equation 14). The data represented by green stars is taken from Leckband and coworkers who probed C-cadherin ectodomains. ${ }^{[156]}$ Linear fit of low unbinding forces (dark grey circles: $k_{\text {off }}=5 * 10^{-3} \mathrm{~s}^{-1}, x_{u}=1.5 \mathrm{~nm}$ ) and high unbinding forces (light grey squares: $k_{\text {off }}=2 * 10^{-5} \mathrm{~s}^{-1}, x_{u}=1.2 \mathrm{~nm}$ )

\footnotetext{
${ }^{87}$ Data included in 155. Fichtner, D., et al. (2012). Quantitative and covalent immobilization of E-Cadherins allows evaluation of their lateral density required for cell adhesion. Manuscript in preparation. Göttingen/Karlsruhe.
} 
A bimodal distribution of unbinding forces can be explained either by participation of more than a single EC15 monomer in forming a non-covalent bond at the surface or by two different interaction sites depending on the molecular orientation. The former explanation is less likely since all force extension curves considered in the spectrum could be fitted with a persistence length of $0.5 \mathrm{~nm}$ following a WLC-model. A persistence length of $0.5 \mathrm{~nm}$ is indicative of a single polypeptide chain, while lateral dimerization might change this value to larger values due to loss in flexibility. Moreover, Chu and coworkers measured essentially an identical binding strength of wild-type cadherin structures acting as monomers or laterally connected dimers (peak force of $(64 \pm 27) \mathrm{pN}$ ). ${ }^{[157]}$ Although the bond strengths of the monomer and cadherin-Fc dimer adhesive complexes are similar, the cadherin-Fc dimer showed a higher probability of binding than a cadherin monomer. The so-called "strand dimer" hypothesis assumes that cadherins adhere through a mechanism involving the mutual binding of Trp2 from the N-terminal EC domain (EC1) of one cadherin to a hydrophobic pocket on the EC1 domain of the opposing protein. Biomembrane force probe measurements indicate, however, that multiple binding states are responsible for the adhesive contact between cadherins. Leckband and coworkers found two weak bonds in the case of rupturing EC1-EC2 fragments with $k_{o f f, 1}=3.9 \mathrm{~s}^{-1}$ and $k_{\text {off, }, 2}=0.019 \mathrm{~s}^{-1}$. In contrast, EC1-EC5 fragments exhibit four different bonds differing in strength and off-rate. It was verified that the different states did not correspond to multiple cross-links. The dominant peak at higher forces displays a substantially smaller off-rate $\left(k_{\text {off }}=(3.9 \pm 0.7) \times 10^{-4} \mathrm{~s}^{-1}\right)$ corresponds well with our findings. In contrast, however, we did not find any dwell-time dependency indicative of reorganization processes on the surfaces. Those processes are probably very fast and cannot be resolved on the ms time scale.

\subsubsection{Impact of trans/cis binding for the EC15/EC15-interaction}

We further calculated the density of cadherin monomers on surface functionalized with $1 \%$ of BG thiol assuming that the amount of immobilized cadherin constructs is the same. We assume that dimer formation of adjacent cadherin monomers through cis conjugation occurs in a critical binding distance. Furthermore, we include a radius of action for each cadherin mon- 
omer to account for the flexibility of the EC15 constructs and the enhanced tendency to establish cis interactions with neighboring monomers. Depending on the surface concentration of monomers, we envision different interaction scenarios Figure 6.7. Below the critical binding distance scenario $A$ is most probable. Above this value scenarios $B$ is conceivable. Around the critical distance, we expect scenario $B$ to be most realistic.

A simple calculation can be performed in order to get a rough estimate of the ratio of monomers to dimers on the EC15 functionalized surfaces with a receptor density controlled by the thiol composition BGT/MT (1:100).
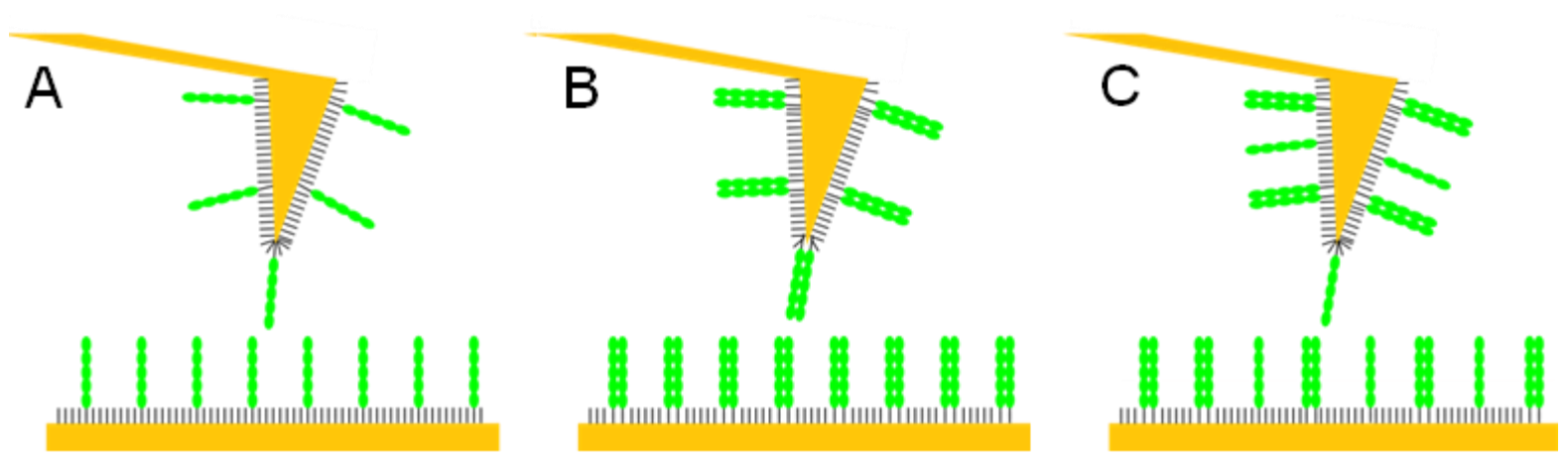

Figure 6.7: Schematic illustration of conceivable trans interaction scenarios that possibly explain the occurrence of two peaks in rupture force histograms of EC15 in the presence of calcium ions. In scenario A, two distinct interaction sites of an individual monomer are probed. In scenario B, two EC15 molecules join laterally to form dimers, which might lead to an increase of interaction forces. A third possibility of interaction is a mixed monomer-dimer setup as depicted in C.

Assuming a monomer surface density of two monomers per $\mathrm{nm}^{2}$ and an action radius of 0.2 $\mathrm{nm}$ of each monomer, we calculate that around $20 \%$ of dimers are formed on the surfaces. If we increase the radius of action to $0.8 \mathrm{~nm}$ for the same monomer coverage, a dimerization value of $99 \%$ is achieved. Thus, we conclude that a scenario similar to the one depicted in C is most probable in our measurements. This in turn could be an explanation for the bimodal distribution that was observed throughout the measurements (Figure 6.5). In order to distinguish between pure monomer interactions and pure dimer interactions cadherin concentrations would have to be reduced to realize scenario $A$ in Figure 6.7 and increased to establish scenario B. A second possibility to interpret the bimodal dejoining force distributions is a sliding mechanism as reported by Leckband and coworkers, which is characterized by a 2step trans binding mechanism. ${ }^{[101]}$ 
A weak interaction between EC1 subunits is characterized by fast kinetics and is followed by a strong kind of interaction between subunits EC3 with slow binding kinetics. It is conceivable that the bimodality is a result of partial strengthening of cadherin interaction upon sliding of ectodomains. The results of chapters 6.1 and 6.2 are included in the work of Fichtner et al. ${ }^{[155]}$

\subsection{Quantification of cadherin monomer-cell interactions}

As described in chapter 3.8.3, we tested the compatibility of the single molecule force spectroscopy setup with cellular cadherin systems. To realize this, we brought an EC15functionalized tip in contact with an adherent and determined rupture forces upon cantilever retraction in order to calculate the interaction strength between EC15 molecules and native E-cadherins on a cell surface (Figure 6.8).

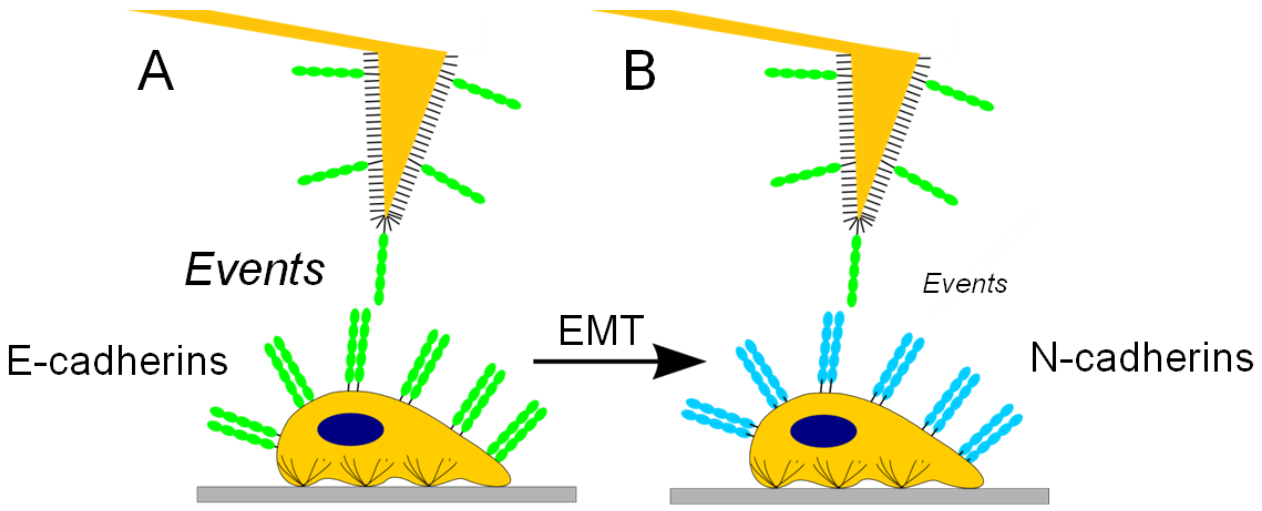

Figure 6.8: Hybrid setups involving interactions probed between EC15 and native cell surfaces before and after epithelial-to-mesenchymal transition (EMT). Upon incubation with TGF- $\beta$ the EMT is induced being associated with a down-regulation of E-cadherin surface structures (green) and an up-regulation of $\mathrm{N}$ cadherin structures (blue). The homomeric E-cadherin interaction is reported to be stronger than the heteromeric E-cadherin- $\mathrm{N}$-cadherin interaction. ${ }^{[158]}$

We performed such measurements on epithelial and on mesenchymal-like cell, which had been treated with TGF- $\beta 1$ prior to the force spectroscopy measurement. TGF- $\beta$ is known to induce the EMT, leading to a down-regulation of E-cadherins and up-regulation of $\mathrm{N}$ cadherins. We probed EC15-epithelial cell interactions as well as EC15-mesenchymal-like cell interactions. Hence, we were able to determine the self-recognition efficiency of cadherin molecules. Interestingly, after $48 \mathrm{~h}$ of TGF- $\beta 1$ stimulation, the number of events decreases to about $50 \%$ in comparison to the untreated samples. Additionally, a decline of the mean rupture is observable from about $35 \mathrm{pN}$ before the transition to about $20 \mathrm{pN}$ reaching the final 
mesenchymal-like state. This decrease of the values can be attributed to a selective cadherin switch from E-Cadherin to N-Cadherin during EMT. ${ }^{[159]}$ Prakasam et al. described heteromeric interactions between $\mathrm{E}$-cadherin and $\mathrm{N}$-Cadherin explaining the observed decline of the rupture forces. ${ }^{[158]}$

To assure that we indeed detect interactions of cadherins, we pre-incubated cells with EDTA resulting in a loss of cell-cell contact density. After adding the chelator agent, the number of events declined to about $5 \%$ in comparison to the untreated samples supporting the assumption that single cadherin bonds are detected. 


\section{Conclusions}

In this work, a membrane probe spectroscopy technique has been developed, which allows monitoring the rupture force of ligand-receptor pairs as a function of bilayer fluidity, loading rate, and ionic strength in the absence of thermal undulations with minimal influence of nonspecific interaction forces (Figure 7.1). Moreover, it offers the possibility to investigate the forced contact between two bilayers, the involved instabilities upon merging, and the retraction process possibly characterized by stochastic unbinding events and the formation of membrane tethers. By equipping a colloidal probe setup with tailored membrane surfaces and by probing specific receptor-ligand interactions, we have shown that even simple systems of weak and strong ligand-receptor interactions bear rich physics and demand intricate models to explain the resulting new membrane structures.

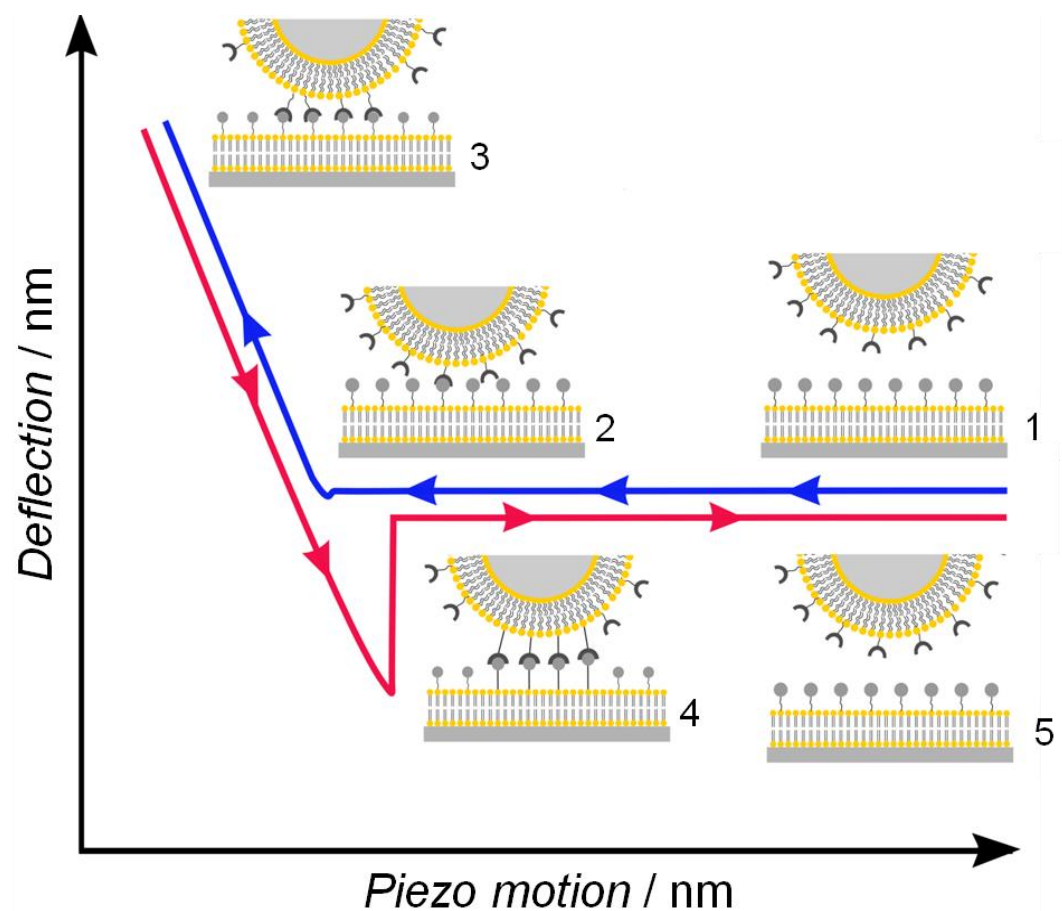

Figure 7.1: Probing multiple biomolecular interactions in a membrane probe force spectroscopy setup. ${ }^{88}$ In contrast to a single molecule setup (Figure 4.2), this setup allows for the investigation of multiple binding processes and of bond cluster formation during the approach-retraction cycle. As solid-supported artificial membranes are used, the fluidity and functionalization of the surfaces can be controlled and, hence, allows mimicking cell membrane features.

\footnotetext{
${ }^{88}$ Adapted from 65. Bizzarri, A. R. and Cannistraro, S. (2010). "The application of atomic force spectroscopy to the study of biological complexes undergoing a biorecognition process." Chemical Society Reviews 39(2): 734-749.
} 
After probing membranes functionalized with strongly interacting compounds represented by the $\mathrm{Ni}^{2+}-\mathrm{NTA}-\mathrm{H} 6$ interaction, we suspect that hemifusion of the outer membrane leaflets mediated by molecular recognition is a highly probable pathway after membrane contact finally leading to the formation of membrane nanotubes.

Moreover, we observed another feature of the membrane probe setup equipped with $\mathrm{Ni}^{2+}-$ NTA-H6 interaction system. We found that the force profiles extracted from these measurements exhibit a shape similar to cell-substrate and cell-cell spectroscopy measurements (Figure 7.2):

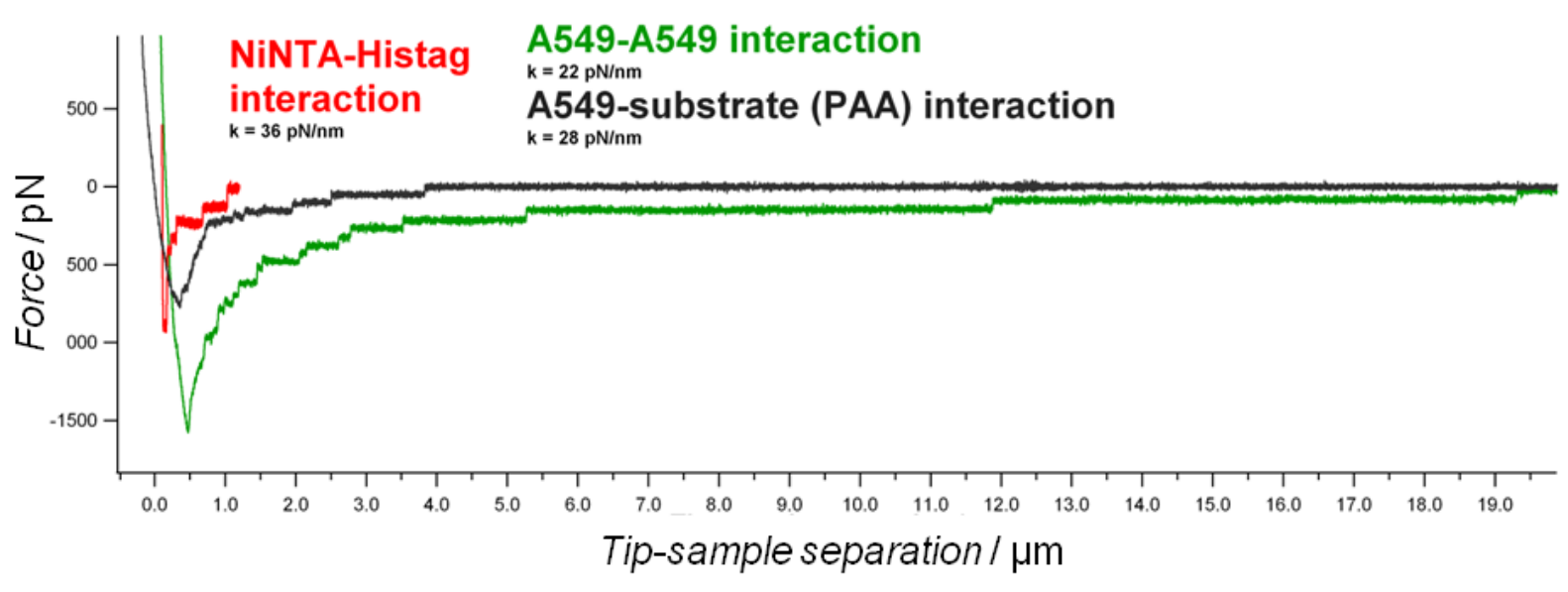

Figure 7.2: Comparison of retraction curves extracted from force spectroscopy measurements involving $\mathrm{Ni}^{2+}$-NTA-H6 interaction (red curve), a cell-cell interaction (green curve), and a cell-substrate interaction (black curve). ${ }^{89}$ The force profile obtained from probing the $\mathrm{Ni}^{2+}$ complexation process resembles the shape of the profiles involving native cells although the magnitude of tether elongation differs by a factor of four (compared to cell-substrate curve) and 12 (compared to cell-cell curve), respectively.

Strikingly, the length of pulled tethers from the artificial system is substantially smaller than in the native systems, which might be due to the fact that cells rely on an enormous membrane reservoir, which can be exhausted for pulling tethers. Moreover, the contact area in the membrane probe setup is much smaller than in setups involving native cells that allow conformal contact.

While probing weak homomeric interactions between disaccharides derived from Microciona prolifera, we found that the weak binding affinity prevents clustering beyond statistical matching and therefore allows observing the bond breakage from individual bonds to very

\footnotetext{
${ }^{89}$ Measurement involving A549 cells performed by David Schneider, University of Göttingen 
small ensembles. Those entities only comprise a few dimers allowing for the measurement of the effect of rebinding that enhances the measured strength at low loading rates.

By equipping the membrane probe setup with a heterodimeric peptide system, we were able to show that the number of successfully formed coiled-coils strongly depends on the lateral organization of peptides in the lipid bilayer. We found that the number of peptide structures available for membrane docking is reduced due to the formation of lipopeptide clusters on the surface as revealed by other methods. The formation of these clusters might be an explanation for reduced fusion efficiency in artificial systems due to a hindered docking of membranes.

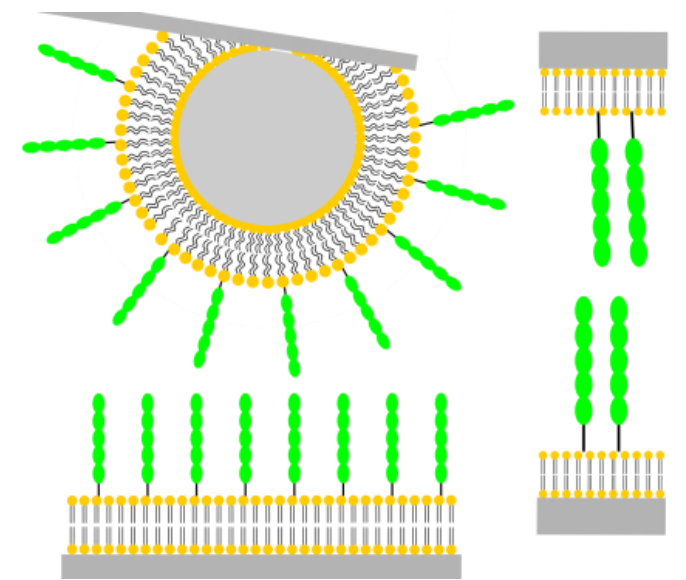

Figure 7.3: Membrane probe spectroscopy setup for the investigation of the homomeric interaction between membrane-bound cadherin structures enabling for the diffusion of cadherin monomers in order to investigate the impact of cadherin oligomerization on the functionality of cadherin adhesion sites. Specific coupling of SNAP-tagged proteins can be performed with a benzylguanine-modified lipid as synthesized by Dr. Andreas Welle. ${ }^{[124]}$

In the context of cell mimics, a possible modification of the system could be the application of soft polymer spheres as substrates for membrane spreading exhibiting a larger contact area as the glass probes at comparable contact forces. A different approach is to equip the membrane probe system with proteins derived from native cells such as the cadherin structures analyzed in chapter 6 . In our studies, we were able to show that the full length ectodomain of the E-cadherin structure is required to perform successful cadherin-mediated recognition. It is still a matter of debate if cadherin monomers are sufficient for efficient binding or if cadherin dimers are needed. In this context, it is conceivable that the membrane probe setup is equipped with cadherin structures via benzylguanine-modified lipids to study whether oligomerization of the protein structures boosts the interaction strength or not (Figure 7.3). 
As proteins are allowed to rearrange in fluid matrices, this setup would allow determining the impact of cadherin organization on the functionality of adherence junctions and desmosomes.

We conclude that the system allows for the study of membrane-membrane interaction from the initial contact and formation of a non-covalent linkage to a possible (hemi) fusion and finally the dynamic action of the bilayers upon retraction creating membrane tethers that display the inherent mechanics of the two joint bilayers. With its defined probe geometry and a great versatility the membrane probe spectroscopy technique can be used to mimic biological interaction systems in order to extract relevant information about adhesion phenomena, possible breakthrough or membrane fusion events as well as about the formation and the lifetime of lipid tethers. Membrane compositions and functionalization, membrane joining forces and times, as well as force ramps acting on the bonds can be varied to investigate interaction systems great detail. Moreover, the impact of lateral organization of molecules on binding strength as well as the effect of inhibitors on an interaction in a lipid environment can be studied with this technique. We consider the membrane probe spectroscopy technique to be a valuable tool to complement traditional methods such as vesicle fusion assays, particle aggregation measurements, and single-molecule spectroscopy setups as well as methods involving native cells. 


\section{Appendices}

\subsection{Abbreviations}

\begin{tabular}{|c|c|}
\hline AC mode & intermittent contact mode \\
\hline AFM & Atomic force microscopy \\
\hline ATR-FTIR & Attenuated total reflection - Fourier transform infrared spectroscopy \\
\hline BCA & Bicinchoninic acid (colorimetric assay for testing protein concentration) \\
\hline $\mathrm{BG}(\mathrm{T})$ & Benzylguanine (thiol(s)) \\
\hline BSA & Bovine serum albumin \\
\hline BY & $\beta$-BODIPY ${ }^{\circledR} 500 / 510 \mathrm{C}_{12}$-HPC, fluorescent lipid \\
\hline CAM(s) & Cell adhesion molecule(s) \\
\hline CLSM & Confocal laser scanning microscopy \\
\hline CMC & Critical micelle concentration \\
\hline CPD & Cytoplasmic domain \\
\hline Cys-tag(ged) & (functionalized with) terminal cysteine residue, for coupling to maleimide functions \\
\hline DAPI & 4',6-diamidino-2-phenylindole \\
\hline DFS & Dynamic force spectroscopy/spectrum \\
\hline DLS & Dynamic light scattering \\
\hline DMPC & 1,2-dimyristoyl-sn-glycero-3-phosphocholine \\
\hline DNA & Deoxyribonucleic acid \\
\hline DOGS-NTA-Ni ${ }^{2+}$ & $\begin{array}{l}\text { 1,2-dioleoyl-sn-glycero-3-succinyl equipped with } \mathrm{Ni}^{2+} \text { chelator } \\
\text { able to coordinate histidine residues }\end{array}$ \\
\hline DOPC & 1,2-dioleoyl-sn-glycero-3-phosphocholine \\
\hline DPPC & 1,2-dipalmitoyl-sn-glycero-3-phosphocholine \\
\hline DPPE & 1,2-dipalmitoyl-sn-glycero-3-phosphoethanolamine \\
\hline DS & Desmosome \\
\hline E-cadherin & Epithelial tissue cadherin \\
\hline EC & Extracellular repeat / subunit of extracellular domain \\
\hline EDTA & Ethylene diamine tetraacetic acid \\
\hline EG & Ethylene glycol \\
\hline EMT & Epithelial-mesenchymal transition \\
\hline ESI-MS & Electron spray ionization - mass spectrometry \\
\hline Fmoc & Fluorenylmethoxycarbonyl protection group \\
\hline FRAP & Fluorescence recovery after photobleaching \\
\hline Fucp & Fucose pyranoside \\
\hline Galp & Galactose pyranoside \\
\hline GFP & Green fluorescent protein \\
\hline GlcpNAc & Acetylated glucopyranosyl residue \\
\hline Gln & Glutamine, amino acid \\
\hline H6 & Short term for $\mathrm{Ac}-\mathrm{H}_{6} \mathrm{GGC}-\mathrm{NH}_{2}$ or $\mathrm{Ac}-\mathrm{H}_{6} \mathrm{WGC}-\mathrm{NH}_{2}$ peptide \\
\hline hAGT & Human $\mathrm{O}^{6}$ alkylguanine DNA alkyl transferase \\
\hline HBS & HEPES buffered saline \\
\hline HBTU & $\begin{array}{l}\text { 2-(1H-benzotriazole-1-yl)-1,1,3,3-tetramethyl-aminium hexafluoro- } \\
\text { phosphate, coupling reagent for peptide synthesis }\end{array}$ \\
\hline HEK & Human embryonic kidney \\
\hline HEPES & 4-(2-hydroxyethyl)-1-piperazineethanesulfonic acid \\
\hline
\end{tabular}




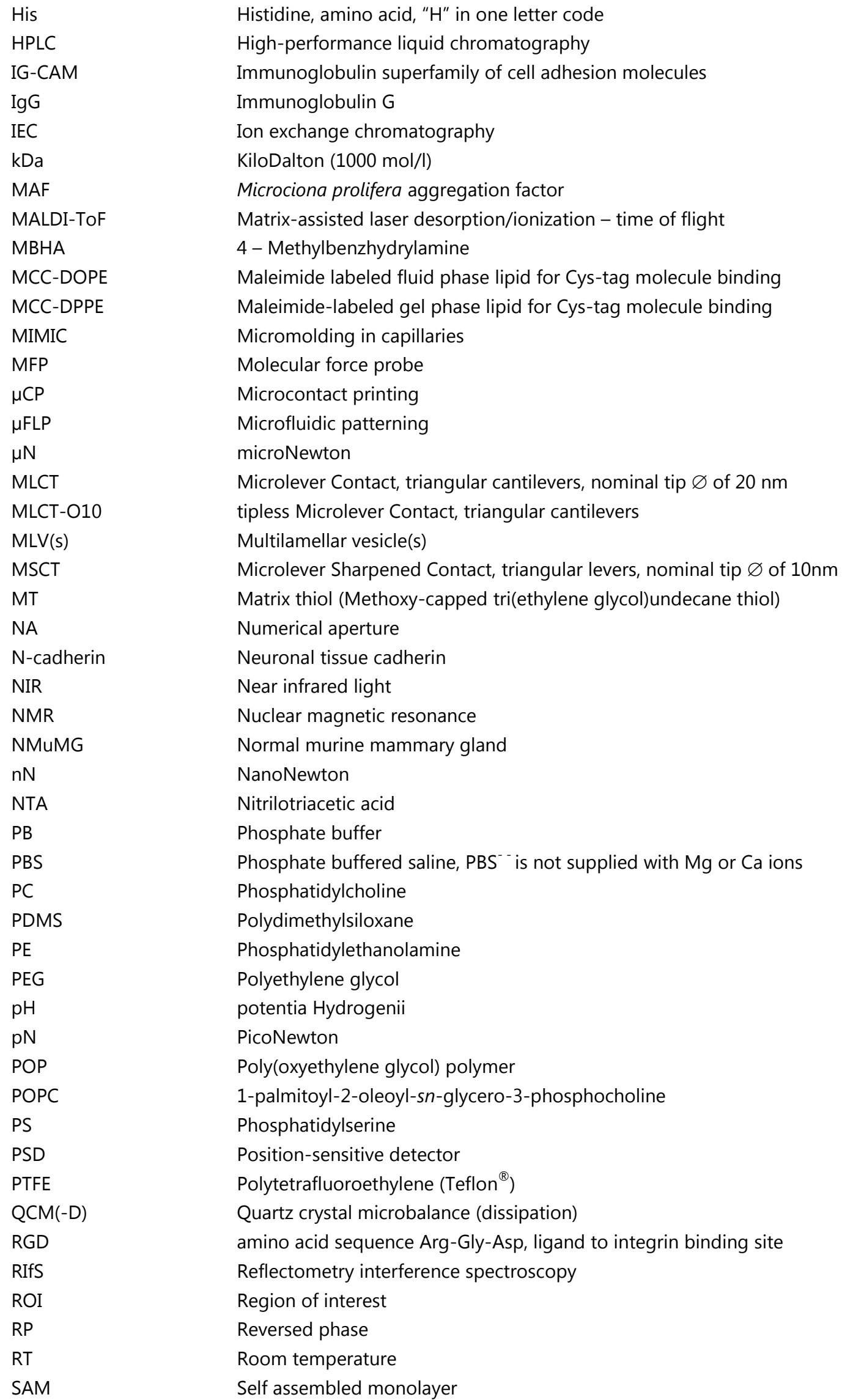




\begin{tabular}{|c|c|}
\hline SDS-PAGE & Sodium dodecyl sulfate polyacrylamide gel electrophoresis \\
\hline SEM & Scanning electron microscopy \\
\hline SFA & Surface force apparatus \\
\hline $\mathrm{S}_{\mathrm{N} 2}$ & Bimolecular nucleophilic substitution \\
\hline SNAP & Trademark of hAGT \\
\hline SNARE & Soluble $\mathrm{N}$-ethylmaleimide-sensitive factor attachment protein receptor \\
\hline SPR & Surface plasmon resonance \\
\hline SSLB & Solid supported lipid bilayer(s) \\
\hline SUV(s) & Small unilamellar vesicle(s) \\
\hline TFA & Trifluoroacetic acid \\
\hline TGF- $\beta$ & Transforming growth factor- $\beta$, cytokine \\
\hline $\mathrm{TM}(\mathrm{D})$ & Transmembrane (domain) \\
\hline TR & Texas Red $®$ DHPE, fluorescent lipid \\
\hline UV & Ultra violet \\
\hline VIS & Visible light \\
\hline WLC & Worm-like chain \\
\hline
\end{tabular}

\subsection{Parameters and constants}

\begin{tabular}{|c|c|}
\hline$d / / d_{\text {Cantilever }}$ & Tip-sample separation / m // Cantilever deflection / m \\
\hline$D$ & Diffusion coefficient $/ \mathrm{m}^{2} \mathrm{~s}^{-1}$ \\
\hline$E$ & Young modulus / $\mathrm{Nm}^{-1}$ \\
\hline$F_{a d} / / F_{\text {load }}$ & Adhesion force / N // load force / N \\
\hline$F_{\text {rup }} / / F_{\text {tether }}$ & Rupture force / N // Tether rupture force / N \\
\hline$f_{r}$ & Resonance frequency / Hz \\
\hline$G^{\circ}$ & Free energy / $\mathrm{kJmol}^{-1}$ \\
\hline invOLS & Inverted optical lever sensitivity / mV ${ }^{-1}$ \\
\hline$\kappa, \kappa_{a p p}$ & bending modulus / Nm // apparent spring constant / $\mathrm{Nm}^{-1}$ \\
\hline$k_{b}$ & Boltzmann constant, $k_{b} \approx 1.38^{\star} 10^{-23} \mathrm{JK}^{-1}$ \\
\hline$k_{c}$ & Stiffness / spring constant of cantilever / $\mathrm{Nm}^{-1}$ \\
\hline$k_{\text {off }}$ & Off rate $/ s^{-1}$ \\
\hline$k_{\text {on }}$ & On rate $/ \mathrm{M}^{-1} \mathrm{~s}^{-1}$ \\
\hline$k_{s} / / k_{s y s}$ & Stiffness / spring constant of single bond // of the system / Nm${ }^{-1}$ \\
\hline$l_{c}$ & Contour length / nm \\
\hline$l_{p}$ & Persistence length / $\mathrm{nm}$ \\
\hline lether & Tether length / m \\
\hline$N / / N_{\text {tether }}$ & Number of bonds, cluster size // Number of tethers \\
\hline$\eta$ & Shear viscosity / $\mathrm{Nm}^{-2} \mathrm{~s}$ \\
\hline OT & Optical thickness / nm \\
\hline psi $\Psi$ & Angle in ellipsometer measurement $/^{\circ}$ \\
\hline$r_{C P}$ & Radius of colloidal probe / $\mu \mathrm{m}$ \\
\hline$R_{f}$ & Retention factor in chromatographical techniques \\
\hline$r_{f}$ & Loading rate $/ \mathrm{Ns}^{-1}$ \\
\hline rms & Root mean square \\
\hline$r_{\text {tether }}$ & Tether radius / $\mathrm{m}$ \\
\hline$r$ & Tip radius / nm \\
\hline
\end{tabular}




$\begin{array}{ll}R_{S} & \text { sample rate } / \mathrm{Hz} \\ \sigma & \text { Lateral tension } / \mathrm{Nm}^{-2} \\ \tau & \text { Lifetime / } \mathrm{s} \\ T / / T_{M} & \text { Temperature } /{ }^{\circ} \mathrm{C} \quad / / \quad \text { Main transition temperature of phospholipids } \\ t & \text { Time } / \mathrm{s} \\ v & \text { Velocity / } \mu \mathrm{ms}^{-1} \\ V & \text { Voltage / Volt } \\ W_{a d} & \text { Surface adhesion energy / J } \\ x & \text { distance / m } \\ x_{u} & \text { Potential barrier width / } \mathrm{m} \\ \varnothing & \text { Diameter }\end{array}$

\subsection{Supplementary information about probed model systems}

\subsubsection{Characterization of the $\mathrm{Ni}^{2+}-\mathrm{NTA}$ - $\mathrm{H} 6$ interaction}

Subsequent binding of $\mathrm{H} 6$ peptides to maleimide-functionalized lipid bilayers and of $\mathrm{Ni}^{2+}$ NTA-functionalized vesicles to $\mathrm{H} 6$ was monitored and quantified via quartz crystal microbalance measurements.(Figure 8.1-A).
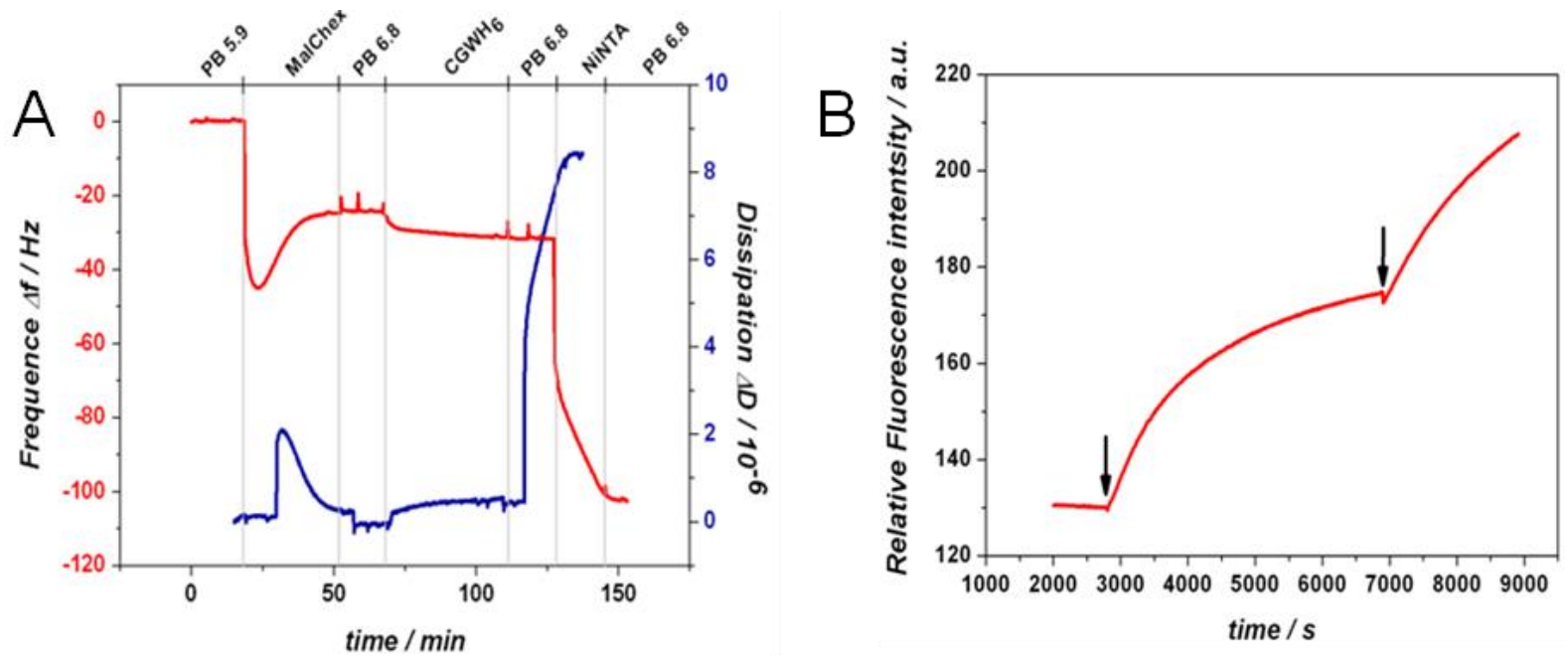

Figure 8.1: Vesicle fusion triggered by $\mathrm{H} 6-\mathrm{Ni}^{2+}-\mathrm{NTA}$ interaction. ${ }^{[75], 90} \mathrm{~A}$ : Coupling of $\mathrm{Ni}^{2+}-\mathrm{NTA}^{2}$ functionalized SUVs to an $\mathrm{H6}^{*}$-functionalized bilayer investigated by QCM-D measurements. After spreading of a DOPC/MCC-DOPE (90:10) bilayer (initial drop of $\Delta f$ ) and the coupling of H6*(second frequency decrease), the frequency drops to $-100 \mathrm{~Hz}$ upon the binding of $\mathrm{Ni}^{2+}-\mathrm{NTA}$-functionalized DOPC (DOPC/ DOGS-NTA- $\left.\mathrm{Ni}^{2+}, 90: 10\right)$. The dissipation rises accordingly implying the attachment of a viscoelastic body. B: Release of TR self-quenching upon addition of $\mathrm{Ni}^{2+}$-NTA-functionalized vesicles (DOPC/ DOGS-NTA$\mathrm{Ni}^{2+}$ (98:2); black arrows) to a population of $\mathrm{H6}^{*}$-functionalized vesicles doped with TR labeled lipids (DOPC/MCC-DOPE-H6*/TR, 88:2:10). While fluorescence intensity is constantly low at high fluorophor concentrations due to self-quenching, TR emission increases upon addition of non-labeled vesicles functionalized with $\mathrm{Ni}^{2+}$-NTA due to lipid mixing.

\footnotetext{
${ }^{90}$ Images reprinted from [75] with the permission of Elsevier.
} 
Texas Red self-quenching assays were used to determine the amount of fusion of $\mathrm{Ni}^{2+}-\mathrm{NTA}-$ doped vesicles with $\mathrm{H6}$-functionalized vesicles.(Figure 8.1-B) The synthesis and purification of Ac- $\mathrm{H}_{6}$ WGC- $\mathrm{NH}_{2}\left(\mathrm{H}^{*}\right)$ was carried out by Rabea Keller.

\subsubsection{EC15 and EC12 proteins}

Figure 8.2 shows the Western Blot analysis of the $50 \mathrm{kDa}$ protein EC12 and the $120 \mathrm{kDa}$ protein $E C 15$. In the case of $E C 12$, fractions 43 to 52 were collected for the purification process. For the purification of EC15, fractions 39 to 49 were collected.

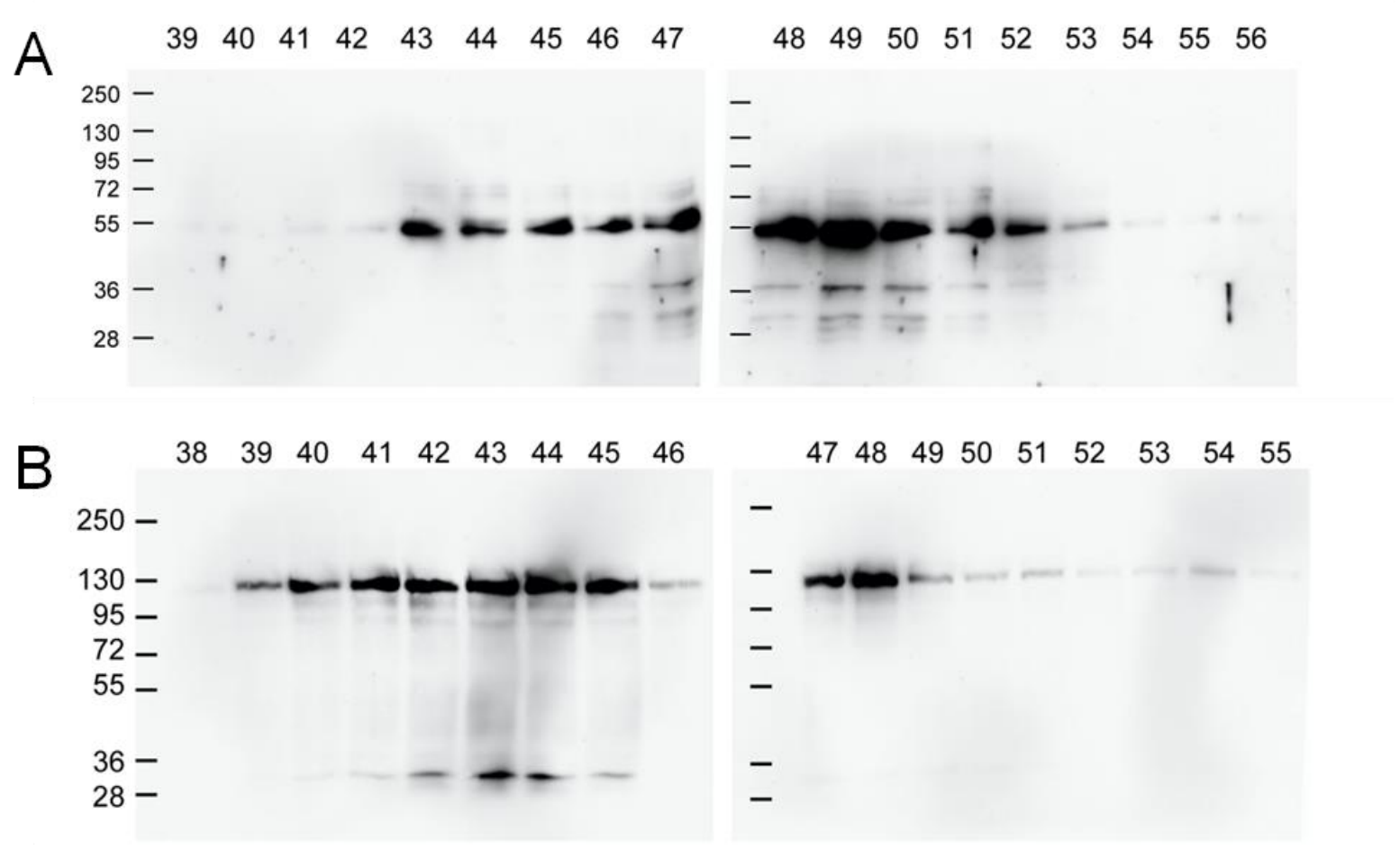

Figure 8.2: Western Blot Analysis of EC12 and EC15 proteins. ${ }^{91}$ For the purification of the $50 \mathrm{kDa} E C 12$ protein, fractions 43 to 52 were collected (A). In the case of the $120 \mathrm{kDa}$ proteins EC15, fractions 39 to 49 of the $120 \mathrm{kDa}$ protein were collected (B).

Expression, characterization, and purification of the proteins were performed by our collaborators Dr. Sinem Engin and Dr. Dagmar Fichtner in the group of Prof. Doris Wedlich in Karlsruhe.

\footnotetext{
${ }^{91}$ Protein expression, purification and characterization performed by Dr. Sinem Engin and Dagmar Fichtner, former members of the Wedlich group, Karlsruhe, usage of images permitted by Dagmar Fichtner. 


\subsection{3 (Non-)sulfated Microciona prolifera disaccharides}

Synthesis and purification of the Microciona prolifera-derived disaccharides was performed by our collaborators Ella Kriemen and Dr. Christian Brand in the group of Dr. Daniel B. Werz in Göttingen. Purification of the non-sulfated disaccharide (10-Mercapto-4-thia-dec-1-yl 2acetamido-2-deoxy- $\beta$-D-glucopyranosyl-(1 $\rightarrow 3)-\alpha$-L-fucopyranoside, synthetic pathway and structure depicted in Figure 8.3) afforded colorless oil with an $R_{f}$ value of $0.81\left(\mathrm{CH}_{2} \mathrm{Cl}_{2} / \mathrm{MeOH}\right.$, $4: 1)^{92}$

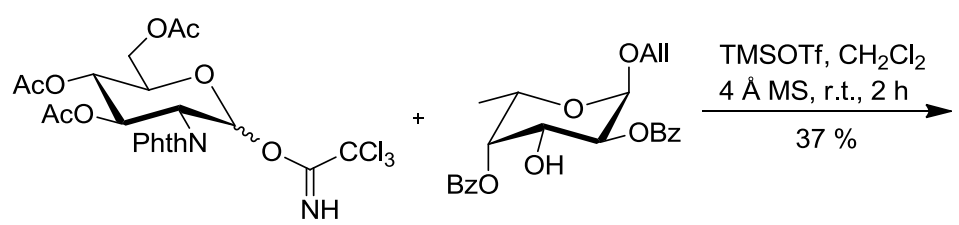

3

4 $\mathrm{BuOH}, 90^{\circ} \mathrm{C}, 23 \mathrm{~h}$

2. $\mathrm{Ac}_{2} \mathrm{O}$, pyridine, r.t. $1.5 \mathrm{~d}$

3. $\mathrm{NH}_{2} \mathrm{Me}, \mathrm{EtOH}$ r.t., $6 \mathrm{~d}$

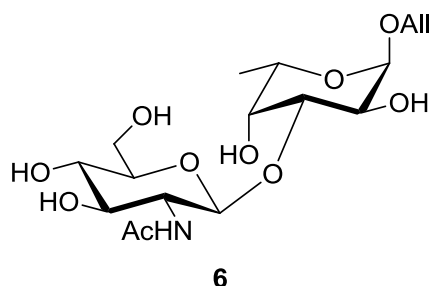

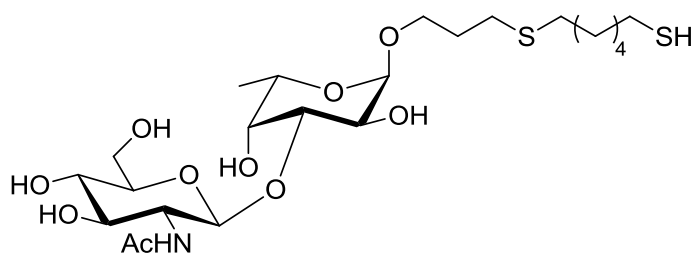

1

Figure 8.3: Synthetic pathway of the non-sulfated disaccharide "1" (10-Mercapto-4-thia-dec-1-yl 2Acetamido-2-deoxy- $\beta$-D-glucopyranosyl-(1 $\rightarrow 3)$ - $\alpha$-L-fucopyranoside). ${ }^{93}$

The substances were characterized by proton $\left({ }^{1} \mathrm{H}-\mathrm{NMR}\right)$ and carbon $\left({ }^{13} \mathrm{C}-\mathrm{NMR}\right) \mathrm{NMR}$ spectra.

\footnotetext{
${ }^{92}$ For detailed information about the synthesis and purification of disaccharides the reader is referred to the supplementary material of reference Lorenz et al., 2012.

${ }^{93}$ Synthesis and purification of compounds was carried out by Christian Brand and Ella Kriemen, group of Dr. D. B. Werz, Göttingen., images reprinted from [92] with the permission of the American Chemical Society.
} 


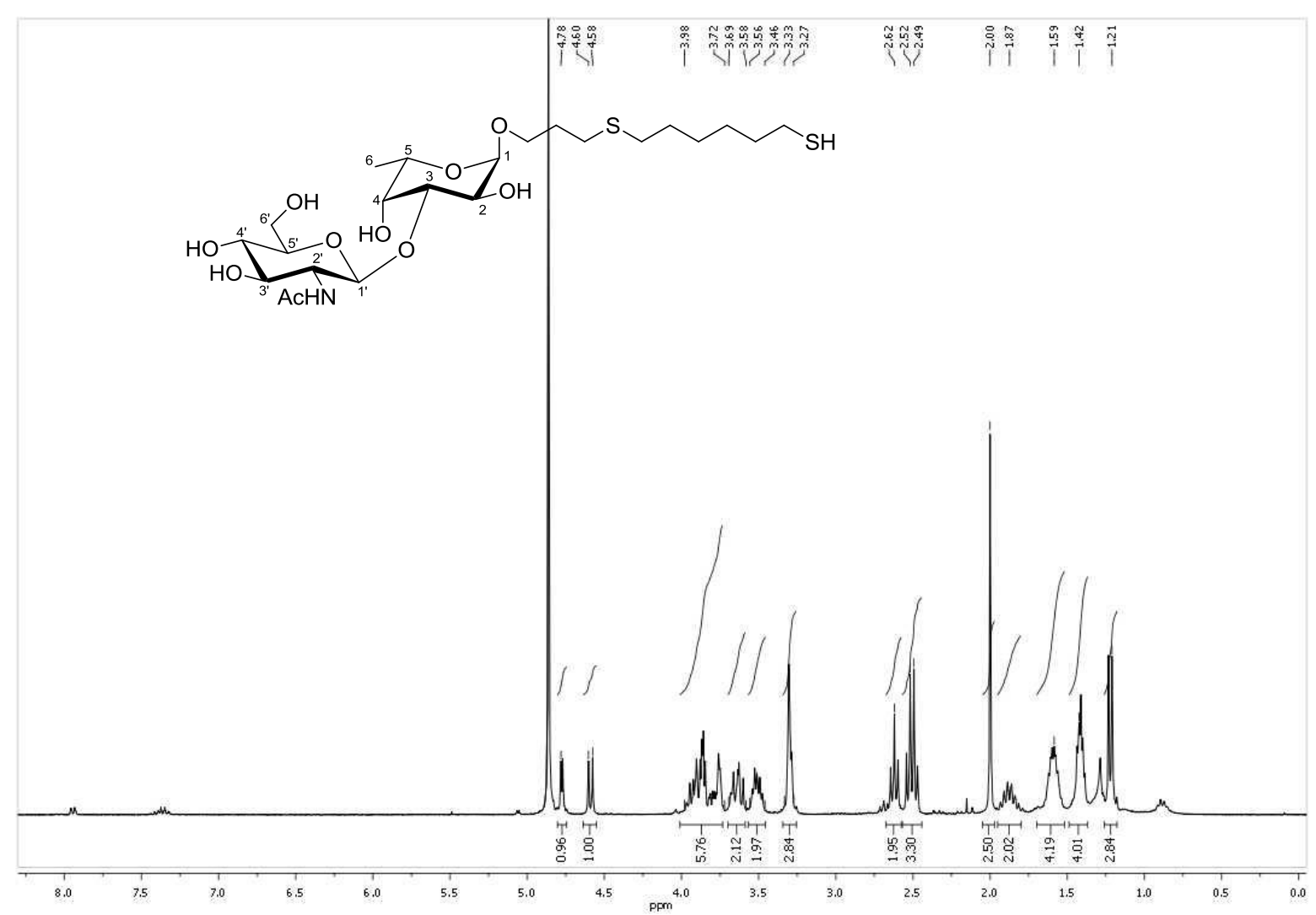

Figure 8.4: ${ }^{1} \mathrm{H}-\mathrm{NMR}\left(300 \mathrm{MHz}, \mathrm{CD}_{3} \mathrm{OD}\right)$ spectrum of the 10-Mercapto-4-thia-dec-1-yl 2-Acetamido-2deoxy- $\beta$-D-glucopyranosyl- $(1 \rightarrow 3)$ - $\alpha$-L-fucopyranoside " 1 ". 93

Chemical shift values for the non-sulfated disaccharide $\left({ }^{1} \mathrm{H}-\mathrm{NMR}\right.$ : Figure $8.4,{ }^{13} \mathrm{C}-\mathrm{NMR}$ : Figure 8.5) were determined to be:

${ }^{1} \mathrm{H}-\mathrm{NMR}\left(300 \mathrm{MHz}, \mathrm{CD}_{3} \mathrm{OD}\right): \delta=1.21(\mathrm{~d}, J=6.5 \mathrm{~Hz}, 3 \mathrm{H}), 1.42\left(\mathrm{~m}_{\mathrm{c}}, 4 \mathrm{H}\right), 1.59\left(\mathrm{~m}_{\mathrm{c}}, 4 \mathrm{H}\right), 1.88$ $\left(m_{c}, 2 \mathrm{H}\right), 2.00\left(\mathrm{~s}, 3 \mathrm{H}, \mathrm{NH}(\mathrm{CO}) \mathrm{CH}_{3}\right), 2.51\left(\mathrm{~m}_{\mathrm{c}}, 4 \mathrm{H}\right), 2.62(\mathrm{t}, J=7.1 \mathrm{~Hz}, 2 \mathrm{H}), 3.27-3.33(\mathrm{~m}, 2 \mathrm{H})$, 3.46-3.56 (m, $2 \mathrm{H}), 3.58-3.69(\mathrm{~m}, 2 \mathrm{H}), 3.72-3.98(\mathrm{~m}, 6 \mathrm{H}), 4.59\left(\mathrm{~d}, J=8.2 \mathrm{~Hz}, 1 \mathrm{H}, 1^{\prime}-\mathrm{H}\right), 4.77$ (d, J = $3.5 \mathrm{~Hz}, 1 \mathrm{H}, 1-\mathrm{H})$ ppm. 


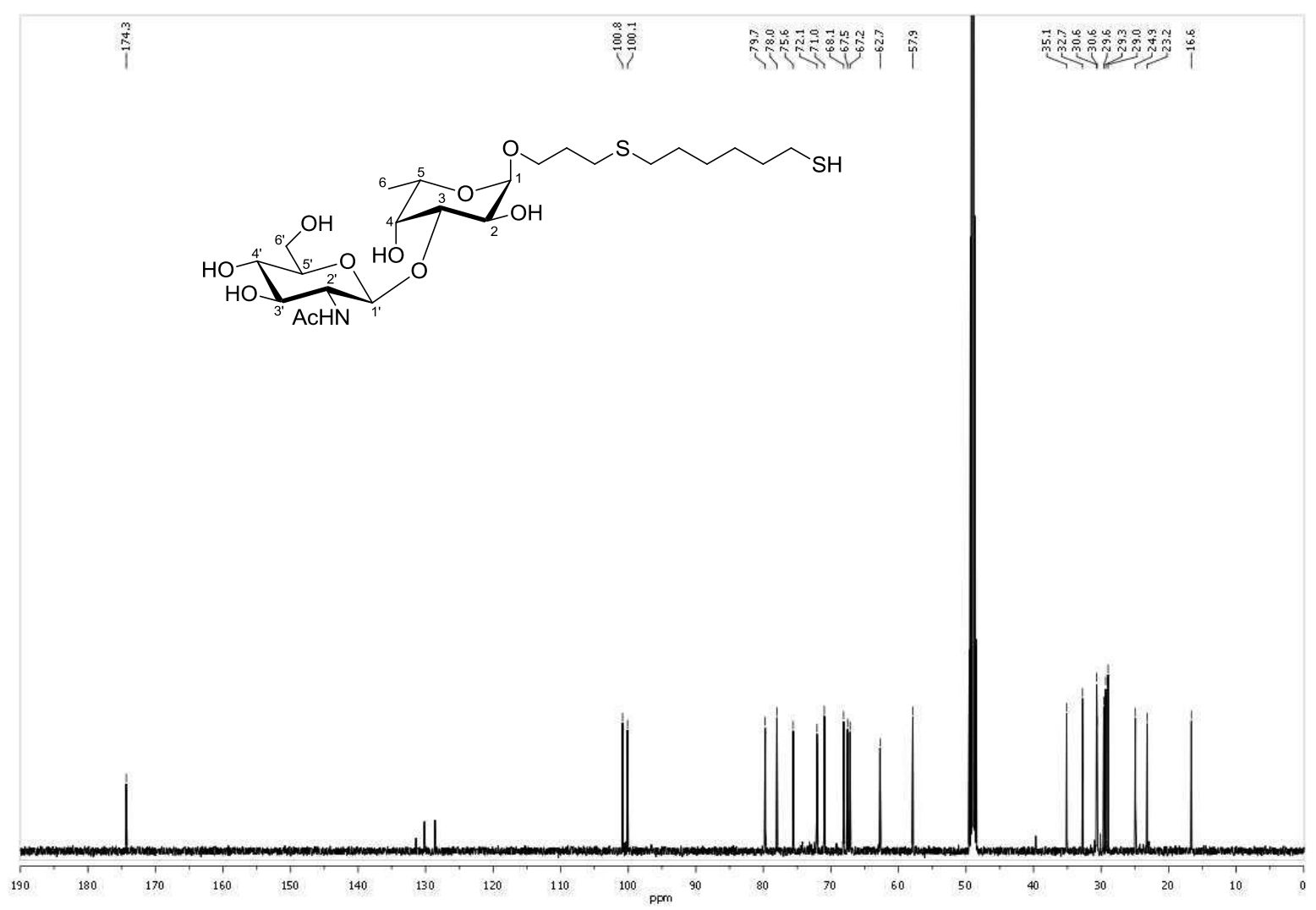

Figure 8.5: ${ }^{13} \mathrm{C}-\mathrm{NMR}\left(125 \mathrm{MHz}, \mathrm{CD}_{3} \mathrm{OD}\right)$ spectrum of the 10-Mercapto-4-thia-dec-1-yl 2-Acetamido-2deoxy- $\beta$-D-glucopyranosyl-(1 $\rightarrow 3)$ - $\alpha$-L-fucopyranoside " 1 ". 93

${ }^{13} \mathrm{C}-\mathrm{NMR}\left(125 \mathrm{MHz}, \mathrm{CD}_{3} \mathrm{OD}\right): \delta=16.6$ (C-6), $23.2\left(\mathrm{NH}(\mathrm{CO}) \mathrm{CH}_{3}\right), 24.9,29.1,29.4,29.7,30.7$, 30.8, 32.8, $35.2\left(\mathrm{OCH}_{2}\left(\mathrm{CH}_{2}\right)_{2} \mathrm{~S}\left(\mathrm{CH}_{2}\right)_{6} \mathrm{SH}\right), 57.9,62.8,67.3,67.7,68.2,71.1,72.1,75.7,78.2,79.9$ (C-2', C-3', C-4', C-5', C-6', C-2, C-3, C-4, C-5, OCH $\left(_{2}\left(\mathrm{CH}_{2}\right)_{2} \mathrm{~S}\left(\mathrm{CH}_{2}\right)_{6} \mathrm{SH}\right), 100.3$ (C-1), 101.0 (C-1'), $174.3\left(\mathrm{NH}(\mathrm{CO}) \mathrm{CH}_{3}\right)$ ppm. HRMS (ESI): $\mathrm{m} / z$ calculated for $\mathrm{C}_{23} \mathrm{H}_{43} \mathrm{NO}_{10} \mathrm{~S}_{2} \mathrm{Na}$ : 580.2221; found: 580.2228 . 
The purification of the sulfated disaccharide (Sodium salt of (10-Mercapto-4-thia-dec-1-yl-2acetamido-2-deoxy-3-O-sulfonato- $\beta$ )-D-glucopyranosyl-(1 $\rightarrow 3)$ - $\alpha$-L-fucopyranoside, synthetic pathway and structure depicted in Figure 8.6) afforded colorless oil with an $R_{f}$ value of 0.88 $\left(\mathrm{CH}_{2} \mathrm{Cl}_{2} / \mathrm{MeOH}, 4: 1\right){ }^{94}$

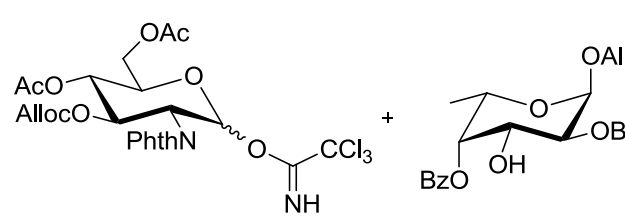

7
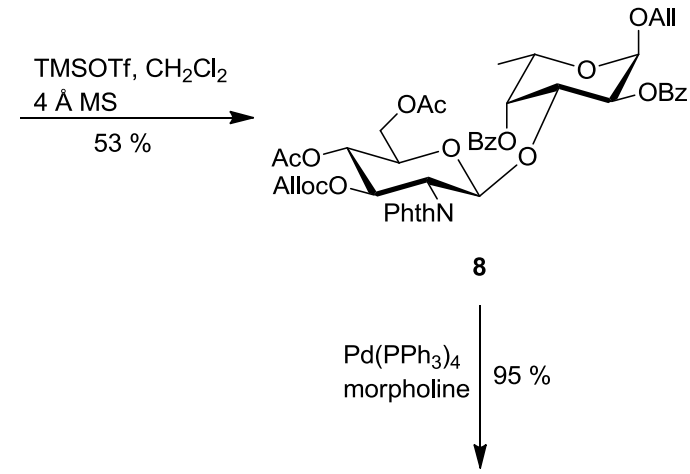

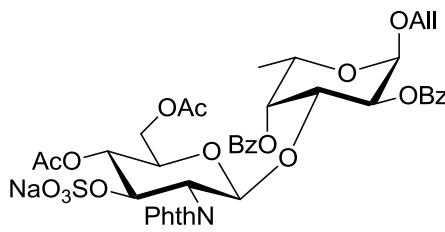

10
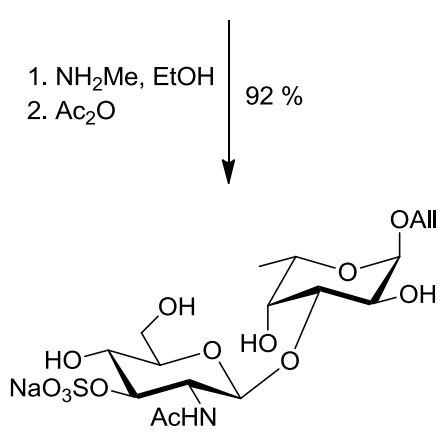

11
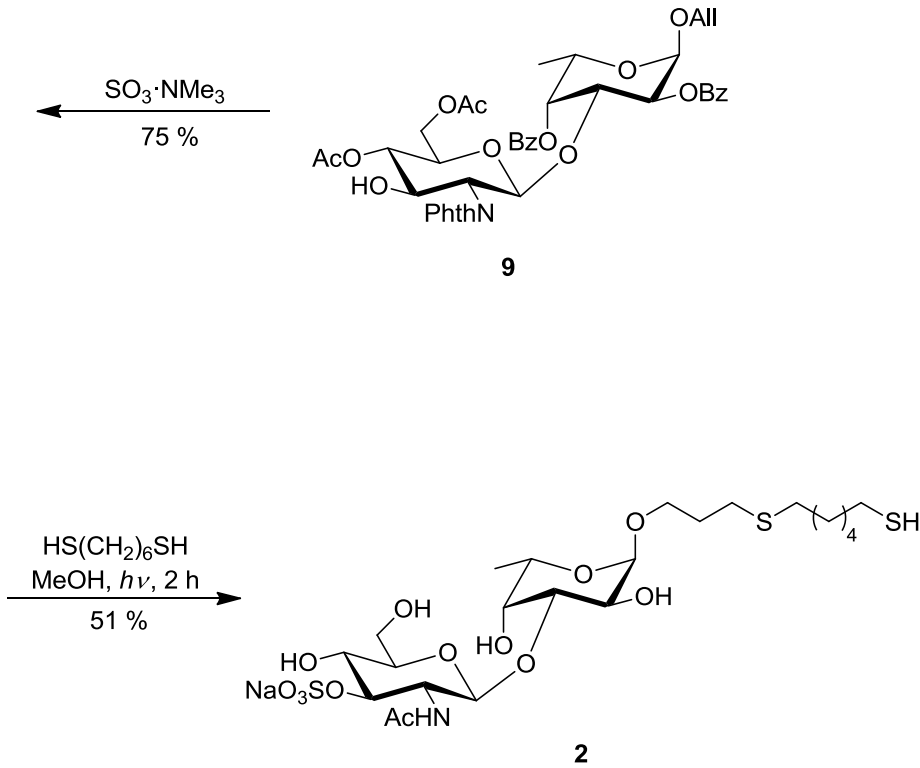

Figure 8.6: Synthetic pathway of the sulfated disaccharide "2"(sodium salt of (10-Mercapto-4-thia-dec-1yl-2-acetamido-2-deoxy-3-O-sulfonato- $\beta$-D-glucopyranosyl-(1 $\rightarrow 3)$ - $\alpha$-L-fucopyranoside) ${ }^{95}$

Chemical shift values for the sulfated disaccharide were determined to be:

${ }^{1} \mathrm{H}-\mathrm{NMR}\left(300 \mathrm{MHz}, \mathrm{CD}_{3} \mathrm{OD}\right.$, Figure 8.7): $\delta=1.22(\mathrm{~d}, J=6.7 \mathrm{~Hz}, 3 \mathrm{H}), 1.42\left(\mathrm{~m}_{\mathrm{c}}, 4 \mathrm{H}\right), 1.59\left(\mathrm{~m}_{\mathrm{c}}\right.$ $4 \mathrm{H}), 1.88\left(\mathrm{~m}_{\mathrm{c}}, 2 \mathrm{H}\right), 2.00\left(\mathrm{~s}, 3 \mathrm{H}, \mathrm{NH}(\mathrm{CO}) \mathrm{CH}_{3}\right), 2.51\left(\mathrm{~m}_{\mathrm{c}}, 4 \mathrm{H}\right), 2.62(\mathrm{t}, J=7.1 \mathrm{~Hz}, 2 \mathrm{H}), 3.41(\mathrm{~m}$, $1 \mathrm{H}), 3.47-3.59(\mathrm{~m}, 2 \mathrm{H}), 3.65-3.97(\mathrm{~m}, 8 \mathrm{H}), 4.44(\mathrm{dd}, J=10.4,8.7 \mathrm{~Hz}, 1 \mathrm{H}), 4.73-4.79(\mathrm{~m}, 2 \mathrm{H}$, $\left.1-\mathrm{H}, 1^{\prime}-\mathrm{H}\right) \mathrm{ppm}$.

\footnotetext{
${ }^{94}$ For detailed information about the synthesis and purification of disaccharides the reader is referred to the supplementary material of reference Lorenz et al., 2012.

${ }^{95}$ Synthesis and purification of compounds was carried out by Christian Brand and Ella Kriemen, group of Dr. D. B. Werz, Göttingen, images reprinted from [92] with the permission of the American Chemical Society.
} 


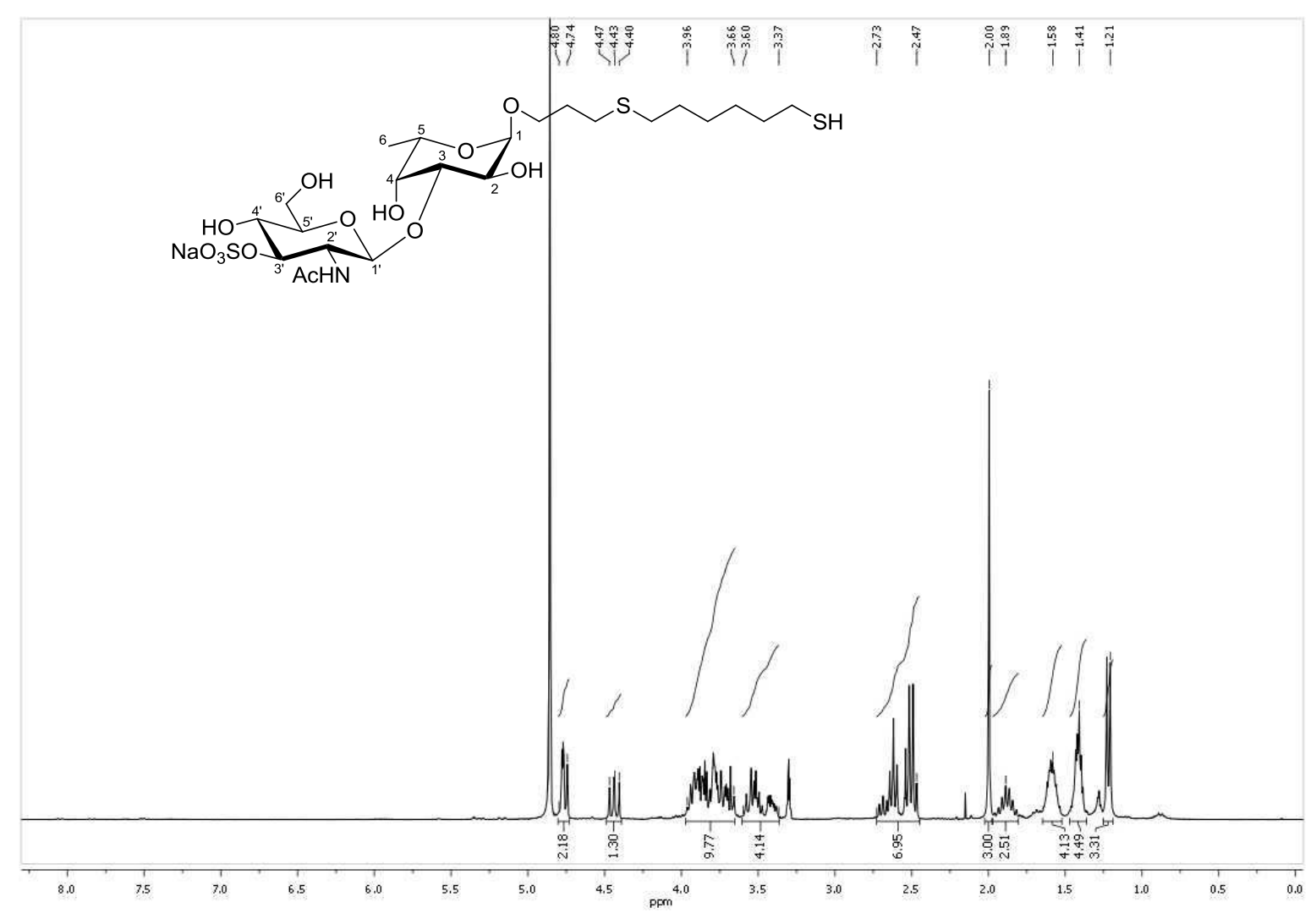

Figure 8.7: ${ }^{1} \mathrm{H}-\mathrm{NMR}$ (300 $\mathrm{MHz}, \mathrm{CD}_{3} \mathrm{OD}$ ) of the sodium salt of (10-Mercapto-4-thia-dec-1-yl-2-Acetamido2-deoxy-3-O-sulfonato- $\beta$-D-glucopyranosyl-(1 $\rightarrow 3)$ - $\alpha$-L-fucopyranoside " 2 ". 95

${ }^{13} \mathrm{C}-\mathrm{NMR}\left(125 \mathrm{MHz}, \mathrm{CD}_{3} \mathrm{OD}\right.$, Figure 8.8): $\delta=16.6(\mathrm{C}-6), 23.3\left(\mathrm{NH}(\mathrm{CO}) \mathrm{CH}_{3}\right), 25.1,29.0,29.3$, 29.6, 30.6, 30.7, 32.7, $35.1\left(\mathrm{OCH}_{2}\left(\mathrm{CH}_{2}\right)_{2} \mathrm{~S}\left(\mathrm{CH}_{2}\right)_{6} \mathrm{SH}\right), 56.9,62.2,67.2,67.6,68.1,70.8,71.0,77.4$, 79.9, 81.7 (C-2, C-3, C-4, C-5, C-2', C-3', C-4', C-5', C-6', OCH$\left.\left(\mathrm{CH}_{2}\right)_{2} \mathrm{~S}\left(\mathrm{CH}_{2}\right)_{6} \mathrm{SH}\right), 100.1,100.8$ $\left(\mathrm{C}-1, \mathrm{C}-1^{\prime}\right), 174.4\left(\mathrm{NH}(\mathrm{CO}) \mathrm{CH}_{3}\right)$ ppm. HRMS (ESI): $\mathrm{m} / \mathrm{z}$ calculated for $\mathrm{C}_{23} \mathrm{H}_{42} \mathrm{NNa}_{2} \mathrm{O}_{13} \mathrm{~S}_{3}$ : 682.1608; found: 682.1607 . 


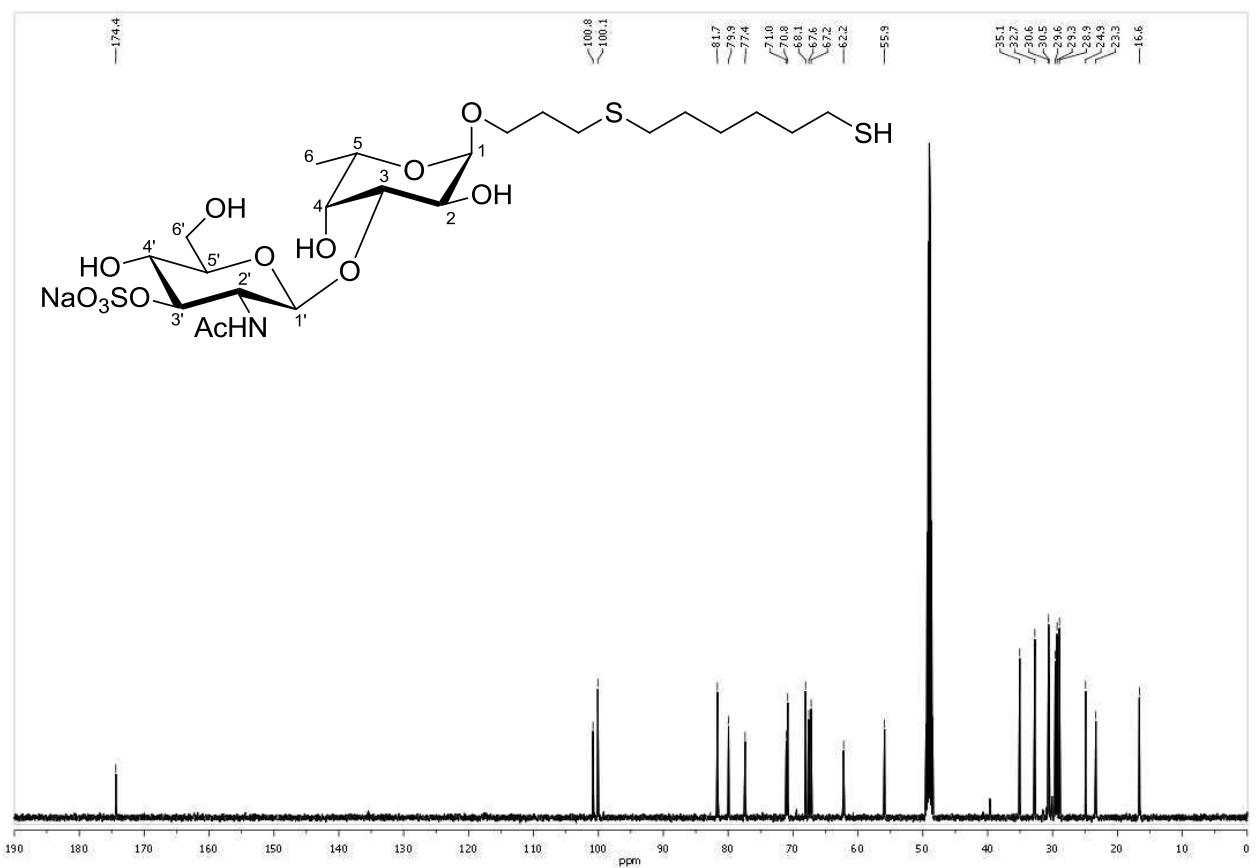

Figure 8.8: ${ }^{13} \mathrm{C}-\mathrm{NMR}\left(125 \mathrm{MHz}, \mathrm{CD}_{3} \mathrm{OD}\right)$ of the sodium salt of (10-Mercapto-4-thia-dec-1-yl-2-Acetamido2-deoxy-3-O-sulfonato- $\beta$-D-glucopyranosyl-( $\rightarrow 3)$ - $\alpha$-L-fucopyranoside " 2 ". 95

\subsection{Cantilevers}

The following table gives an overview over the cantilevers used in this study:

\begin{tabular}{|c|c|c|c|c|c|c|c|}
\hline Name & Manufacturer & $\begin{array}{c}\text { Nom. } \\
k_{c} / \mathrm{Nm}^{-1}\end{array}$ & $\begin{array}{l}f_{R} / \\
\mathbf{k H z}\end{array}$ & $r_{\text {tip }} / \mathrm{nm}$ & Material & Lever/tip shape & $\begin{array}{c}l_{\text {lever }} / \\
\mu \mathrm{m}\end{array}$ \\
\hline $\begin{array}{l}\text { Bio-Lever, } \\
\text { long lever (B) }\end{array}$ & $\begin{array}{l}\text { Olympus, } \\
\text { Hamburg }\end{array}$ & 0.06 & 13 & 30 & $\begin{array}{l}\text { SiN, Au coat- } \\
\text { ing (tip incl.) }\end{array}$ & $\begin{array}{l}\text { Rectangular, in- } \\
\text { dented tip }\end{array}$ & 100 \\
\hline C-lever MSCT & \multirow{5}{*}{$\begin{array}{l}\text { Bruker, } \\
\text { Camarillo }\end{array}$} & \multirow{2}{*}{0.01} & \multirow{2}{*}{$4-10$} & $\begin{array}{l}\text { nom.: } 10 \\
\text { max.: } 40\end{array}$ & \multirow{5}{*}{$\begin{array}{c}\text { SiN, back side: } \\
\text { gold }\end{array}$} & Triangular, rotated & \multirow{3}{*}{310} \\
\hline C-lever MLCT & & & & $\begin{array}{l}\text { nom. } 20 \\
\text { max.: } 60\end{array}$ & & tip & \\
\hline $\begin{array}{c}\text { C-lever } \\
\text { MLCT-OW }\end{array}$ & & 0.01 & $4-10$ & - & & $\begin{array}{c}\text { Triangular, } \\
\text { no lever }\end{array}$ & \\
\hline E-lever MSCT & & \multirow{2}{*}{0.1} & \multirow{2}{*}{$26-50$} & $\begin{array}{l}\text { nom.: } 10 \\
\max .: 40\end{array}$ & & \multirow{2}{*}{$\begin{array}{c}\text { Triangular, rotated } \\
\text { tip }\end{array}$} & \multirow{2}{*}{140} \\
\hline E-lever $M L C T$ & & & & $\begin{array}{l}\text { nom. } 20 \\
\max .: 60\end{array}$ & & & \\
\hline
\end{tabular}

Table 2: Cantilever characteristics. Values for the nominal spring constants $\boldsymbol{k}_{c}$, the nominal resonance frequency $f_{R}$, the nominal tip radius $r_{t i p}$ as well as the cantilever material, its shape and length are given.

A Force Spectroscopy Setup to Mimic Cellular Interaction Processes 
MLCT/MSCT cantilevers were used for AFM imaging (C-lever: contact mode, E-lever: AC mode), whereas Bio-Lever (B-lever) and MLCT-OW levers were used for force spectroscopy measurements.

\subsection{Buffer systems}

The following buffer systems were used for the different setups:

$\mathrm{Ni}^{2+}-\mathrm{NTA}-\mathrm{H} 6$

PB 5.9

$50 \mathrm{mM} \mathrm{Na}_{2} \mathrm{HPO}_{4}, \mathrm{pH}=5.9$ (adjusted with $\mathrm{H}_{3} \mathrm{PO}_{4}$ )

PB 6.8 $50 \mathrm{mM} \mathrm{Na}_{2} \mathrm{HPO}_{4}, \mathrm{pH}=6.8$ (adjusted with $\mathrm{H}_{3} \mathrm{PO}_{4}$ )

Microciona prolifera disaccharides

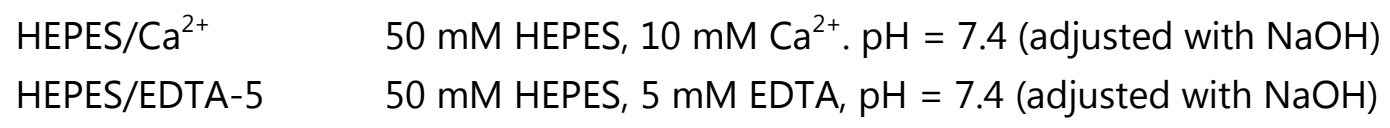

$E C 15$ and $E C 12$

$\mathrm{HBS} / \mathrm{Ca}^{2+}$ $10 \mathrm{mM}$ HEPES, $100 \mathrm{mM} \mathrm{NaCl}, 2 \mathrm{mM} \mathrm{Ca}{ }^{2+}, \mathrm{pH}=7.5$ (adj. with $\mathrm{NaOH}$ )

HBS/EDTA-2 $10 \mathrm{mM}$ HEPES, $100 \mathrm{mM} \mathrm{NaCl}, 2 \mathrm{mM}$ EDTA, $\mathrm{pH}=7.5$ (adj. with $\mathrm{NaOH}$ ) HBS/EDTA-1 10 mM HEPES, 100 mM NaCl, 1 mM EDTA, pH = 7.5 (adj. with $\mathrm{NaOH}$ )

\subsection{Specifications of lipids}

The lipids depicted in Figure 8.9 were used for spreading lipid membranes. DPPC, DOPC, and POPC were used as matrix lipids, whereas MCC-DPPE, MCC-DOPE, and DOGS-NTA- $\mathrm{Ni}^{2+}$ served as receptor lipids for thiol-linked molecules and His-tagged molecules, respectively. Depending on their main transition temperature, the matrix lipids are used to spread membranes with a gel phase or a fluid phase character at room temperature (DPPC: gel phase; DOPC, POPC: fluid phase at RT). 


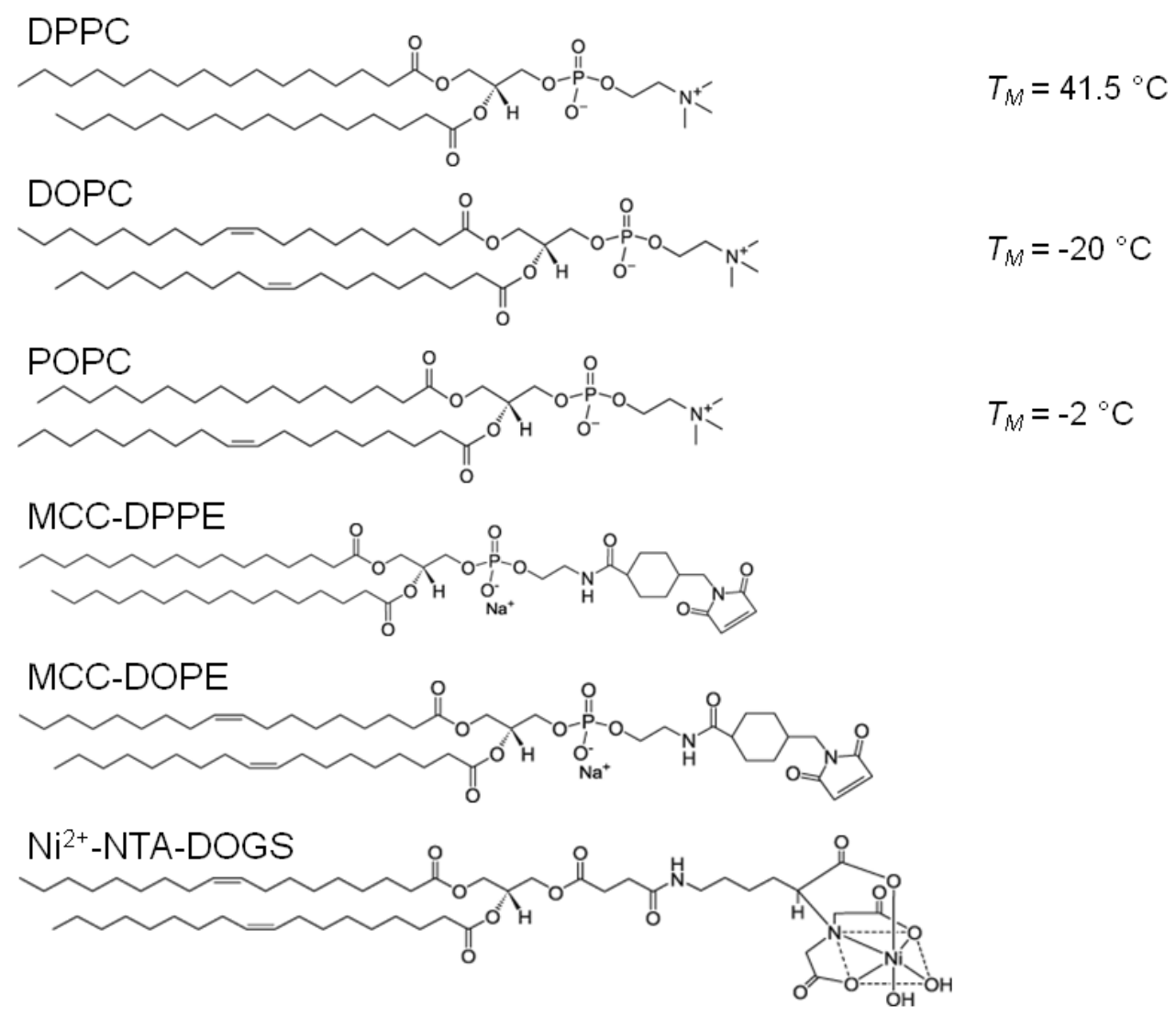

Figure 8.9: Lipid structures as used in this study. ${ }^{96}$ DPPC (1,2-dipalmitoyl-sn-glycero-3-phosphocholine), DOPC (1,2-dioleoyl-sn-glycero-3-phosphocholine), and POPC (1-palmitoyl-2-oleoyl-sn-glycero-3phosphocholine) were used as matrix lipids. Headgroup modified lipids, and DOGS-NTA- $\mathrm{Ni}^{2+}$ were used to covalently bind maleimide-bearing molecules to lipid membranes (MCC-DPPE (1,2-dipalmitoyl-sn-glycero3-phos-phoethanolamine-N-[4-(p-maleimido-methyl)-cyclohexane-carboxamide]) and MCC-DOPE (1,2dioleoyl-sn-glycero-3-phosphoethanol-amine- $\mathrm{N}$-[4-(p-maleimidome-thyl)cyclohexane-carboxamide). $\mathrm{Ni}^{2+}$ DOGS-NTA (1,2-di-(9Z-octadecenoyl)-sn-glycero-3-[(N-(5-amino-1-carboxypentyl)iminodiacetic acid)succinyl] (nickel salt) was used to probe His-tag interactions. All lipids were purchased from Avanti Polar Lipids (Alabaster, AL, USA)

\footnotetext{
${ }^{96}$ Representational structures shown above kindly supplied by Avanti Polar Lipids, Inc., www.avantilipids.com.
} 
A Force Spectroscopy Setup to Mimic Cellular Interaction Processes 


\section{Bibliography}

1. Kasemo, B. (1998). "Biological surface science." Current Opinion in Solid State and Materials Science 3(5): $451-$ 459.

2. Rosso, F., et al. (2004). "From Cell-ECM interactions to tissue engineering." Journal of Cellular Physiology 199(2): 174-180.

3. Judge, D. and Dietz, H. (2005). "Marfan's syndrome." Lancet 366(9501): 1965-1976.

4. van der Flier, A. and Sonnenberg, A. (2001). "Function and interactions of integrins." Cell and Tissue Research 305(3): 285-298.

5. Sackmann, E. and Goennenwein, S. (2006). "Cell Adhesion as Dynamic Interplay of Lock-and-Key, Generic and Elastic Forces." Progress of Theoretical Physics Supplement 165: 78-99.

6. Wegener, J. (2001). Cell Junctions. eLS, John Wiley \& Sons, Ltd.

7. Baoukina, S., et al. (2012). "Molecular Structure of Membrane Tethers." Biophysical Journal 102(8): 1866-1871.

8. Gorter, E. and Grendel, F. (1925). "ON BIMOLECULAR LAYERS OF LIPOIDS ON THE CHROMOCYTES OF THE BLOOD." The Journal of experimental medicine 41(4): 439-443.

9. Singer, S. J. and Nicolson, G. L. (1972). "The fluid mosaic model of the structure of cell membranes." Science 175(4023): 720-731.

10. Kusumi, A., et al. (1993). "Confined lateral diffusion of membrane receptors as studied by single particle tracking (nanovid microscopy). Effects of calcium-induced differentiation in cultured epithelial cells." Biophysical Journal 65(5): 2021-2040.

11. Raedler, J., et al. (1995). "Phenomenology and Kinetics of Lipid Bilayer Spreading on Hydrophilic Surfaces." Langmuir 11(11): 4539-4548.

12. Marsh, D. (2013). Handbook of Lipid Bilayers, CRC Press; 2 edition (January 16, 2013).

13. Cremer, P. S. and Boxer, S. G. (1999). "Formation and Spreading of Lipid Bilayers on Planar Glass Supports." The Journal of Physical Chemistry B 103(13): 2554-2559.

14. Wagner, M. L. and Tamm, L. K. (2000). "Tethered Polymer-Supported Planar Lipid Bilayers for Reconstitution of Integral Membrane Proteins: Silane-Polyethyleneglycol-Lipid as a Cushion and Covalent Linker." Biophysical Journal 79(3): 1400-1414.

15. Mueller, P., et al. (1962). "Reconstitution of Cell Membrane Structure in vitro and its Transformation into an Excitable System." Nature 194(4832): 979-980.

16. Kocun, M., et al. (2011). "Preparation of Solvent-Free, Pore-Spanning Lipid Bilayers: Modeling the Low Tension of Plasma Membranes." Langmuir 27(12): 7672-7680.

17. Tamm, L. K. and McConnell, H. M. (1985). "Supported phospholipid bilayers." Biophysical Journal 47(1): 105113.

18. Watts, T. H., et al. (1984). "Antigen presentation by supported planar membranes containing affinity-purified IAd." Proceedings of the National Academy of Sciences 81(23): 7564-7568. 
19. Bendas, G., et al. (1997). "A liposome-based model system for the simulation of lectin-induced cell adhesion." Biochimica et Biophysica Acta (BBA) - Biomembranes 1325(2): 297-308.

20. Sunshine, C. and McNamee, M. G. (1992). "Lipid modulation of nicotinic acetylcholine receptor function: the role of neutral and negatively charged lipids." Biochimica et Biophysica Acta (BBA) - Biomembranes 1108(2): 240 246.

21. Deserno, M. (2007). Fluid lipid membranes - a primer. Mainz.

22. Scherer, P. G. and Seelig, J. (1989). "Electric charge effects on phospholipid headgroups. Phosphatidylcholine in mixtures with cationic and anionic amphiphiles." Biochemistry 28(19): 7720-7728.

23. Bretscher, M. S. (1972). "Asymmetrical lipid bilayer structure for biological membranes." Nature: New biology 236(61): 11-12.

24. Ziegler, U., et al. (1998). "Preparation of basal cell membranes for scanning probe microscopy." FEBS Letters 436(2): 179-184.

25. Lorenz, B., et al. (2009). "Elasticity Mapping of Pore-Suspending Native Cell Membranes." Small 5(7): 832-838.

26. Simonsson, L., et al. (2011). "Continuous Lipid Bilayers Derived from Cell Membranes for Spatial Molecular Manipulation." Journal of the American Chemical Society 133(35): 14027-14032.

27. Kindermann, M., et al. (2003). "Covalent and Selective Immobilization of Fusion Proteins." Journal of the American Chemical Society 125(26): 7810-7811.

28. Folkers, J. P., et al. (1994). "Phase behavior of two-component self-assembled monolayers of alkanethiolates on gold." The Journal of Physical Chemistry 98(2): 563-571.

29. Love, J. C., et al. (2005). "Self-Assembled Monolayers of Thiolates on Metals as a Form of Nanotechnology." Chemical Reviews 105(4): 1103-1170.

30. Hong, S., et al. (1999). "Multiple Ink Nanolithography: Toward a Multiple-Pen Nano-Plotter." Science 286(5439): 523-525.

31. Falconnet, D., et al. (2006). "Surface engineering approaches to micropattern surfaces for cell-based assays." Biomaterials 27(16): 3044-3063.

32. Silberzan, P., et al. (1991). "Silanation of silica surfaces. A new method of constructing pure or mixed monolayers." Langmuir 7(8): 1647-1651.

33. Headrick, J. E. and Berrie, C. L. (2004). "Alternative Method for Fabricating Chemically Functionalized AFM Tips: Silane Modification of HF-Treated Si3N4 Probes." Langmuir 20(10): 4124-4131.

34. Tsukruk, V. V. and Bliznyuk, V. N. (1998). "Adhesive and Friction Forces between Chemically Modified Silicon and Silicon Nitride Surfaces." Langmuir 14(2): 446-455.

35. Lenhert, S., et al. (2007). "Massively Parallel Dip-Pen Nanolithography of Heterogeneous Supported Phospholipid Multilayer Patterns." Small 3(1): 71-75.

36. Faiss, S., et al. (2007). "Phase Transition of Individually Addressable Microstructured Membranes Visualized by Imaging Ellipsometry." The Journal of Physical Chemistry B 111(50): 13979-13986. 
37. Leckband, D. (2000). "MEASURING THE FORCES THAT CONTROL PROTEIN INTERACTIONS." Annual Review of Biophysics and Biomolecular Structure 29(1): 1-26.

38. Israelachvili, J. N. (1991). Intermolecular and surface forces / Jacob N. Israelachvili. London ; San Diego :, Academic Press.

39. Kukura, P., et al. (2009). "High-speed nanoscopic tracking of the position and orientation of a single virus." Nature Methods 6(12): 923-927.

40. Pollard, T. D. and Borisy, G. G. (2003). "Cellular Motility Driven by Assembly and Disassembly of Actin Filaments." Cell 112(4): 453-465.

41. Friedrichs, J., et al. (2010). "Stimulated single-cell force spectroscopy to quantify cell adhesion receptor crosstalk." Proteomics 10(7): 1455-1462.

42. Helenius, J., et al. (2008). "Single-cell force spectroscopy." Journal of Cell Science 121(11): 1785-1791.

43. Benoit, M. and Gaub, H. E. (2002). "Measuring Cell Adhesion Forces with the Atomic Force Microscope at the Molecular Level." Cells Tissues Organs 172(3): 174-189.

44. Leckband, D., et al. (1995). "Molecular mechanisms determining the strength of receptor-mediated intermembrane adhesion." Biophysical Journal 69(3): 1162-1169.

45. Muller, D., et al. (2009). "Force probing surfaces of living cells to molecular resolution." Nature chemical biology 5(6): 383-390.

46. Hanley, W., et al. (2003). "Single Molecule Characterization of P-selectin/Ligand Binding." Journal of Biological Chemistry 278(12): 10556-10561.

47. Schmitz, J., et al. (2008). "The Viscoelasticity of Membrane Tethers and Its Importance for Cell Adhesion." Biophysical Journal 95(3): 1448-1459.

48. Benoit, M., et al. (2000). "Discrete interactions in cell adhesion measured by single-molecule force spectroscopy." Nat Cell Biol 2(6): 313-317.

49. Smith, A.-S., et al. (2004). "Pulling Tethers from Adhered Vesicles." Physical Review Letters 92(20): 208101.

50. Cuvelier, D., et al. (2005). "Pulling long tubes from firmly adhered vesicles." EPL (Europhysics Letters) 71(6): 1015.

51. Akiyoshi, K., et al. (2003). "Induction of neuron-like tubes and liposome networks by cooperative effect of gangliosides and phospholipids." FEBS Letters 534(1-3): 33-38.

52. Dai, J. and Sheetz, M. P. (1995). "Mechanical properties of neuronal growth cone membranes studied by tether formation with laser optical tweezers." Biophysical Journal 68(3): 988-996.

53. Hochmuth, R. M. and Evans, E. A. (1982). "Extensional flow of erythrocyte membrane from cell body to elastic tether. I. Analysis." Biophysical Journal 39(1): 71-81.

54. Waugh, R. E. (1982). "Surface viscosity measurements from large bilayer vesicle tether formation. I. Analysis." Biophysical Journal 38(1): 19-27.

55. Waugh, R. E. and Hochmuth, R. M. (1987). "Mechanical equilibrium of thick, hollow, liquid membrane cylinders." Biophysical Journal 52(3): 391-400. 
56. Hochmuth, F. M., et al. (1996). "Deformation and flow of membrane into tethers extracted from neuronal growth cones." Biophysical Journal 70(1): 358-369.

57. Sun, M., et al. (2005). "Multiple Membrane Tethers Probed by Atomic Force Microscopy." Biophysical Journal 89(6): 4320-4329.

58. Krieg, M., et al. (2008). "A bond for a lifetime: employing membrane nanotubes from living cells to determine receptor-ligand kinetics." Angewandte Chemie (International ed.) 47(50): 9775-9777.

59. Chernomordik, L. V., et al. (2006). "Membranes of the world unite!" The Journal of cell biology 175(2): 201-207.

60. Farsad, K. and Camilli, P. D. (2003). "Mechanisms of membrane deformation." Current Opinion in Cell Biology 15(4): 372-381.

61. Chernomordik, L. and Kozlov, M. (2008). "Mechanics of membrane fusion." Nature Structural \& Molecular Biology 15(7): 675-683.

62. Abdulreda, M. H. and Moy, V. T. (2007). "Atomic Force Microscope Studies of the Fusion of Floating Lipid Bilayers." Biophysical Journal 92(12): 4369-4378.

63. Leckband, D. E., et al. (1993). "Role of calcium in the adhesion and fusion of bilayers." Biochemistry 32(4): 11271140 .

64. Anderson, T. H., et al. (2009). "Formation of Supported Bilayers on Silica Substrates." Langmuir 25(12): 69977005 .

65. Bizzarri, A. R. and Cannistraro, S. (2010). "The application of atomic force spectroscopy to the study of biological complexes undergoing a biorecognition process." Chemical Society Reviews 39(2): 734-749.

66. Abdulreda, M. H., et al. (2009). "Pulling force generated by interacting SNAREs facilitates membrane hemifusion." Integrative Biology 1(4): 301-310.

67. Hochuli, E., et al. (1987). "New metal chelate adsorbent selective for proteins and peptides containing neighbouring histidine residues." Journal of Chromatography A 411(0): 177-184.

68. Schmitt, J., et al. (1993). "Affinity purification of histidine-tagged proteins." Molecular Biology Reports 18(3): 223-230.

69. Schmitt, L., et al. (1994). "Synthesis and Characterization of Chelator-Lipids for Reversible Immobilization of Engineered Proteins at Self-Assembled Lipid Interfaces." Journal of the American Chemical Society 116(19): 84858491.

70. Schmitt, L., et al. (2000). "A Metal-Chelating Microscopy Tip as a New Toolbox for Single-Molecule Experiments by Atomic Force Microscopy." Biophysical Journal 78(6): 3275-3285.

71. Verbelen, C., et al. (2007). "The NTA-His6 bond is strong enough for AFM single-molecular recognition studies." Journal of Molecular Recognition 20(6): 490-494.

72. Lauer, S. A. and Nolan, J. P. (2002). "Development and characterization of Ni-NTA-bearing microspheres." Cytometry 48(3): 136-145.

73. Conti, M., et al. (2000). "How Strong Is the Coordination Bond between a Histidine Tag and NiNitrilotriacetate? An Experiment of Mechanochemistry on Single Molecules." Angewandte Chemie International Edition 39(1): 215-218. 
74. Liu, W. and Parpura, V. (2009). "Single Molecule Probing of SNARE Proteins by Atomic Force Microscopy." Annals of the New York Academy of Sciences 1152(1): 113-120.

75. Lorenz, B., et al. (2010). "Colloidal probe microscopy of membrane-membrane interactions: From ligandreceptor recognition to fusion events." Biophysical Chemistry 150(1-3): 54-63.

76. DeFrees, S. A., et al. (1996). "Sialyl Lewis x Liposomes as a Multivalent Ligand and Inhibitor of E-Selectin Mediated Cellular Adhesion." Journal of the American Chemical Society 118(26): 6101-6104.

77. Ellis, J. (1786). The natural history of many curious and uncommon zoophytes, collected from various parts of the globe / by the late John Ellis; systematically arranged and described by the late Daniel Solander. London :, Printed for Benjamin White and Son, and Peter Elmsly.

78. Bucior, I., et al. (2004). "Carbohydrate-carbohydrate interaction provides adhesion force and specificity for cellular recognition." Journal of Cell Biology 165(4): 529-537.

79. Thakur, N. L. and Muller, W. E. G. (2004). "Biotechnological potential of marine sponges." Current Science 86(11): 1506-1512.

80. Wijffels, R. H. (2008). "Potential of sponges and microalgae for marine biotechnology." Trends in Biotechnology 26(1): 26-31.

81. Imai, Y., et al. (1993). "Sulphation requirement for GlyCAM-1, an endothelial ligand for L-selectin." Nature 361(6412): 555-557.

82. Brandley, B. K., et al. (1993). "Structure-function studies on selectin carbohydrate ligands. Modifications to fucose, sialic acid and sulphate as a sialic acid replacement." Glycobiology 3(6): 633-641.

83. Spillmann, D., et al. (1995). "Characterization of a Novel Sulfated Carbohydrate Unit Implicated in the Carbohydrate-Carbohydrate-mediated Cell Aggregation of the Marine Sponge Microciona prolifera." Journal of Biological Chemistry 270(10): 5089-5097.

84. Bucior, I. and Burger, M. M. (2004). "Carbohydrate-carbohydrate interactions in cell recognition." Current Opinion in Structural Biology 14(5): 631-637.

85. De Souza, A. C., et al. (2009). "Adhesion forces in the self-recognition of oligosaccharide epitopes of the proteoglycan aggregation factor of the marine sponge Microciona prolifera." Glycoconjugate journal 26(4): 457465 .

86. Jarchow, J., et al. (2000). "Supramolecular Structure of a New Family of Circular Proteoglycans Mediating Cell Adhesion in Sponges." Journal of Structural Biology 132(2): 95-105.

87. Fernandez-Busquets, X., et al. (2009). "Self-Recognition and $\mathrm{Ca}(2+)$-Dependent Carbohydrate-Carbohydrate Cell Adhesion Provide Clues to the Cambrian Explosion." Molecular Biology and Evolution 26(11): 2551-2561.

88. Garcia-Manyes, S., et al. (2006). "Proteoglycan mechanics studied by single-molecule force spectroscopy of allotypic cell adhesion glycans." Journal of Biological Chemistry 281(9): 5992-5999.

89. De Souza, A. C., et al. (2005). "Gold Glyconanoparticles as Probes to Explore the Carbohydrate-Mediated SelfRecognition of Marine Sponge Cells." ChemBioChem 6(5): 828-831.

90. De Souza, A. C., et al. (2004). "Synthesis of gold glyconanoparticles: Possible probes for the exploration of carbohydrate-mediated self-recognition of marine sponge cells." European Journal of Organic Chemistry(21): 4323-4339. 
91. Haseley, S. R., et al. (2001). "Carbohydrate self-recognition mediates marine sponge cellular adhesion." Proceedings of the National Academy of Sciences 98(16): 9419-9424.

92. Lorenz, B., et al. (2012). "A Model System for Cell Adhesion Mediated by Weak Carbohydrate-Carbohydrate Interactions." Journal of the American Chemical Society 134(7): 3326-3329.

93. Coombe, D. R., et al. (1987). "A role for sulfated polysaccharide recognition in sponge cell aggregation." Experimental Cell Research 170(2): 381-401.

94. Schuy, S., et al. (2008). "In situ Synthesis of Lipopeptides as Versatile Receptors for the Specific Binding of Nanoparticles and Liposomes to Solid-Supported Membranes." Small 4(7): 970-981.

95. Pähler, G., et al. (2012). "Coiled-Coil Formation on Lipid Bilayers Implications for Docking and Fusion Efficiency." Biophysical Journal 103(11): 2295-2303.

96. Lorenz, B., et al. (2013). "Impact of peptide clustering on unbinding forces in the context of fusion mimetics." Biochem. Biophys. Res. Commun. 430(3): 938-943.

97. Robson Marsden, H., et al. (2009). "A Reduced SNARE Model for Membrane Fusion." Angewandte Chemie International Edition 48(13): 2330-2333.

98. Jahn, R., et al. (2003). "Membrane Fusion." Cell 112(4): 519-533.

99. Fernandez, I., et al. (1998). "Three-Dimensional Structure of an Evolutionarily Conserved N-Terminal Domain of Syntaxin 1A." Cell 94(6): 841-849.

100. Sutton, R. B., et al. (1998). "Crystal structure of a SNARE complex involved in synaptic exocytosis at 2.4[thinsp]A resolution." Nature 395(6700): 347-353.

101. Bayas, M. V., et al. (2006). "Lifetime Measurements Reveal Kinetic Differences between Homophilic Cadherin Bonds." Biophysical Journal 90(4): 1385-1395.

102. Brasch, J., et al. (2012). "Thinking outside the cell: how cadherins drive adhesion." Trends in cell biology.

103. Harrison, O. J., et al. (2011). "The Extracellular Architecture of Adherens Junctions Revealed by Crystal Structures of Type I Cadherins." Structure 19(2): 244-256.

104. Engin, S., et al. (2010). "Benzylguanine Thiol Self-Assembled Monolayers for the Immobilization of SNAP-tag Proteins on Microcontact-Printed Surface Structures." Langmuir 26(9): 6097-6101.

105. Binnig, G., et al. (1986). "Atomic Force Microscope." Physical Review Letters 56(9): 930-933.

106. Künneke, S. (2003). Kontaktmechanik und Strukturierung von festkörperunterstützen Lipidmembranen. Institut für Physikalische Chemie, Fachbereich Chemie und Pharmazie. Mainz, Johannes Gutenberg-Universität in Mainz.

107. Butt, H.-J., et al. (2005). "Force measurements with the atomic force microscope: Technique, interpretation and applications." Surface Science Reports 59(1-6): 1-152.

108. Kasas, S. and Dietler, G. (2008). "Probing nanomechanical properties from biomolecules to living cells." Pflügers Archiv European Journal of Physiology 456(1): 13-27.

109. Janshoff, A., et al. (2000). "Force Spectroscopy of Molecular Systems-Single Molecule Spectroscopy of Polymers and Biomolecules." Angewandte Chemie International Edition 39(18): 3212-3237. 
110. Kocun, M. (2011). Mechanical properties of pore-spanning membranes prepared from giant vesicles. Institute for Physical Chemistry. Göttingen, Georg August University.

111. Axelrod, D., et al. (1976). "Mobility measurement by analysis of fluorescence photobleaching recovery kinetics." Biophysical Journal 16(9): 1055-1069.

112. Schaaf, P., et al. (1987). "Reflectometry as a technique to study the adsorption of human fibrinogen at the silica/solution interface." Langmuir 3(6): 1131-1135.

113. Schaaf, P., et al. (1986). "VARIABLE ANGLE REFLECTOMETRY FOR STRUCTURAL STUDIES OF ADSORBED LAYERS." Revue de physique appliquée 21(11): 741-745.

114. Gauglitz, G. (2005). "Direct optical sensors: principles and selected applications." Analytical and Bioanalytical Chemistry 381(1): 141-155.

115. Gauglitz, G. (2010). "Direct optical detection in bioanalysis: an update." Analytical and Bioanalytical Chemistry 398(6): 2363-2372.

116. Kaspar, S., et al. (1999). "Influence of relative humidity in sensing halogenated hydrocarbons with Reflectometric Interference Spectroscopy (RIfS)." Fresenius' journal of analytical chemistry 363(2): 193-196.

117. Belmont, A.-S., et al. (2007). "Molecularly imprinted polymer films for reflectometric interference spectroscopic sensors." Biosensors and Bioelectronics 22(12): 3267-3272.

118. Brecht, A., et al. (1992). "DIRECT MONITORING OF ANTIGEN-ANTIBODY INTERACTIONS BY SPECTRAL INTERFEROMETRY." Sensors and actuators. B, Chemical 6(1-3): 96-100.

119. Möhrle, B., et al. (2006). "Label-free characterization of cell adhesion using reflectometric interference spectroscopy (RIfS)." Analytical and Bioanalytical Chemistry 384(2): 407-413.

120. Schwartz, M. P., et al. (2006). "The Smart Petri Dish: A Nanostructured Photonic Crystal for Real-Time Monitoring of Living Cells." Langmuir 22(16): 7084-7090.

121. MacDonald, R. C., et al. (1991). "Small-volume extrusion apparatus for preparation of large, unilamellar vesicles." Biochimica et Biophysica Acta (BBA) - Biomembranes 1061(2): 297-303.

122. Carpino, L. A. and Han, G. Y. (1972). "9-Fluorenylmethoxycarbonyl amino-protecting group." The Journal of Organic Chemistry 37(22): 3404-3409.

123. Coin, I., et al. (2007). "Solid-phase peptide synthesis: from standard procedures to the synthesis of difficult sequences." Nat. Protocols 2(12): 3247-3256.

124. Engin, S. (2010). Funktionalisierung strukturierter Oberflächen mit E-Cadherin-Ektodomänen. Fakultät für Chemie und Biowissenschaften. Karlsruhe, Karlsruher Institut für Technologie (KIT).

125. Spratt, T. E. and Delossantos, H. (1992). "Reaction of O6-alkylguanine-DNA alkyltransferase with O6methylguanine analogues: evidence that the oxygen of O6-methylguanine is protonated by the protein to effect methyl transfer." Biochemistry 31(14): 3688-3694.

126. Shin, H. (2007). "Fabrication methods of an engineered microenvironment for analysis of cell-biomaterial interactions." Biomaterials 28(2): 126-133.

127. Kumar, A., et al. (1994). "PATTERNING SELF-ASSEMBLED MONOLAYERS - APPLICATIONS IN MATERIALS SCIENCE." Langmuir 10(5): 1498-1511. 
128. Keppler, A., et al. (2003). "A general method for the covalent labeling of fusion proteins with small molecules in vivo." Nat Biotech 21(1): 86-89.

129. Xu, J., et al. (2009). "TGF-[beta]-induced epithelial to mesenchymal transition." Cell Res 19(2): 156-172.

130. Noy, A. (2011). "Force spectroscopy 101: how to design, perform, and analyze an AFM-based single molecule force spectroscopy experiment." Current Opinion in Chemical Biology 15(5): 710-718.

131. Bell, G. (1978). "Models for the specific adhesion of cells to cells." Science 200(4342): 618-627.

132. Evans, E. and Ritchie, K. (1997). "Dynamic strength of molecular adhesion bonds." Biophysical Journal 72(4): 1541-1555.

133. Bustamante, C., et al. (1994). "Entropic elasticity of lambda-phage DNA." Science 265(5178): 1599-1600.

134. van Zwol, P. J., et al. (2008). "Roughness of Microspheres for Force Measurements." Langmuir 24(14): 75287531.

135. Pera, I., et al. (2004). "Using the atomic force microscope to study the interaction between two solid supported lipid bilayers and the influence of synapsin I." Biophys. J. 87(4): 2446-2455.

136. Hertz, H. (1882). Ueber die Berührung fester elastischer Körper. Journal für die reine und angewandte Mathematik (Crelle's Journal). 1882: 156.

137. Künneke, S., et al. (2004). "Scrutiny of the Failure of Lipid Membranes as a Function of Headgroups, Chain Length, and Lamellarity Measured by Scanning Force Microscopy." Biophysical Journal 86(3): 1545-1553.

138. Williams, J. M., et al. (1996). "Determination of Single-Bond Forces from Contact Force Variances in Atomic Force Microscopy." Langmuir 12(5): 1291-1295.

139. Mey, I., et al. (2009). "Local Membrane Mechanics of Pore-Spanning Bilayers." Journal of the American Chemical Society 131(20): 7031-7039.

140. Nassoy, P., et al. (2008). "Nanofluidics in cellular tubes under oscillatory extension." EPL (Europhysics Letters) 84(1): 18004.

141. Weikl, T. R., et al. (2009). "Adhesion of membranes via receptor-ligand complexes: Domain formation, binding cooperativity, and active processes." Soft Matter 5(17): 3213-3224.

142. Smith, A.-S. and Sackmann, E. (2009). "Progress in Mimetic Studies of Cell Adhesion and the Mechanosensing." Chemphyschem 10(1): 66-78.

143. Fenz, S. F., et al. "Inter-membrane adhesion mediated by mobile linkers: Effect of receptor shortage." Soft Matter 7(3): 952-962.

144. Smith, A.-S. and Seifert, U. (2007). "Vesicles as a model for controlled (de-)adhesion of cells: a thermodynamic approach." Soft Matter 3(3): 275-289.

145. Fenz, S. F., et al. "Switching from Ultraweak to Strong Adhesion." Advanced Materials 23(22-23): 2622-+.

146. Bell, G. I., et al. (1984). "CELL-ADHESION - COMPETITION BETWEEN NONSPECIFIC REPULSION AND SPECIFIC BONDING." Biophysical journal 45(6): 1051-1064.

147. Seifert, U. (2000). "Rupture of multiple parallel molecular bonds under dynamic loading." Physical Review Letters 84(12): 2750-2753. 
148. Erdmann, T. (2005). Stochastic dynamics of adhesion clusters under force.

149. Paehler, G., et al. (2012). Thermodynamics and kinetics of parallel and antiparallel coiled-coil formation on lipid bilayers - implication for docking and fusion. Manuscript in preparation. Göttingen.

150. Pettersen, E. F., et al. (2004). "UCSF Chimera-A visualization system for exploratory research and analysis." Journal of Computational Chemistry 25(13): 1605-1612.

151. Lang, T., et al. (2001). "SNAREs are concentrated in cholesterol-dependent clusters that define docking and fusion sites for exocytosis." EMBO J 20(9): 2202-2213.

152. Sieber, J. J., et al. (2007). "Anatomy and Dynamics of a Supramolecular Membrane Protein Cluster." Science 317(5841): 1072-1076.

153. Liu, T., et al. (2005). "SNARE-Driven, 25-Millisecond Vesicle Fusion In Vitro." Biophysical Journal 89(4): 24582472.

154. Lorenz, B., et al. (2013). "Impact of peptide clustering on unbinding forces in the context of fusion mimetics." Biochemical and Biophysical Research Communications 430(3): 938-943.

155. Fichtner, D., et al. (2012). Quantitative and covalent immobilization of E-Cadherins allows evaluation of their lateral density required for cell adhesion. Manuscript in preparation. Göttingen/Karlsruhe.

156. Leckband, D. (2008). "Beyond structure: mechanism and dynamics of intercellular adhesion." Biochemical Society Transactions 36: 213-220.

157. Zhang, Y., et al. (2009). "Resolving cadherin interactions and binding cooperativity at the single-molecule level." Proceedings of the National Academy of Sciences 106(1): 109-114.

158. Prakasam, A. K., et al. (2006). "Similarities between heterophilic and homophilic cadherin adhesion." Proceedings of the National Academy of Sciences of the United States of America 103(42): 15434-15439.

159. Maeda, M., et al. (2005). "Cadherin switching: essential for behavioral but not morphological changes during an epithelium-to-mesenchyme transition." Journal of Cell Science 118(5): 873-887. 
A Force Spectroscopy Setup to Mimic Cellular Interaction Processes 


\section{Publications}

Lorenz, B., G. Paehler, A. Janshoff (2013). "Impact of Peptide Clustering on Unbinding Forces in the Context of Fusion Mimetics" Biochem. Biophys. Res. Commun., 430(3): 938-43

Lorenz, B., Álvarez de Cienfuegos, L. et al. (2012). "Model System for Cell Adhesion Mediated by Weak Carbohydrate-Carbohydrate Interactions". Journal of the American Chemical Society 134(7): 3326-3329.

Lorenz, B., Keller, R. et al. (2010). "Colloidal Probe Microscopy of Membrane-Membrane Interactions: From Ligand-Receptor Recognition to Fusion Events" Biophysical Chemistry 150(1-3): 54-63. 
